
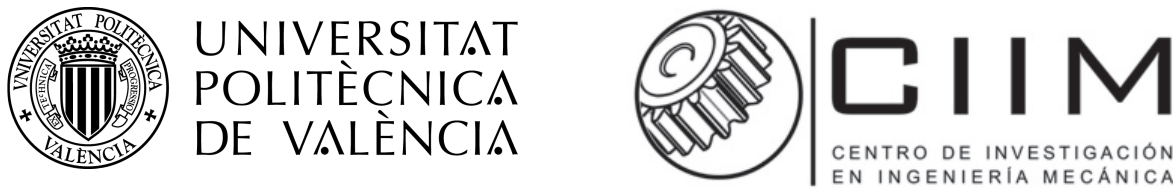

Universitat Politècnica de VAlÈnCiA

Centro de Investigación en Ingeniería Mecánica - CIIM

PhD THESIS

\title{
Assessment of head injury risk caused by impact using finite element models
}

\author{
Author: \\ Marta Palomar Toledano \\ Supervisor: \\ Eugenio Giner Maravilla
}

November 26, 2019 


\section{Agradecimientos}

No sería justo presentar esta Tesis sin mencionar a todas aquellas personas de mi entorno que de un modo u otro también han sido partícipes de ella.

A lo largo de estos más de tres años, he pasado por diferentes etapas de muchos cambios que me han hecho evolucionar como persona (quiero creer que para bien). Lo que más me alegra de este proceso, es que las personas que me han acompañado en él son las mismas que me han acompañado en gran parte de mi vida.

En primer lugar, mi familia. No solo les agradezco la religiosa manutención que me han proporcionado (y que, ahora que vivo sola, puedo apreciar mejor) sino sobre todo el apoyo que me han dado tanto en las buenas como en las malas situaciones. Siempre he querido que mis padres se sientan orgullosos de mí por mis logros, pero ahora entiendo que soy yo quien debe estar orgullosa de ellos, porque poca cosa habría conseguido sin su apoyo.

La otra parte fundamental a quien debo el haber terminado esta Tesis son mis amigos. Todos ellos han sabido ayudarme a desconectar en los momentos más duros y a alegrarse conmigo con las buenas noticias. Los días de trabajo son mucho mejores si sabes que al salir te esperan, en el bar Nuevo Cuenca, esas caras de toda la vida que poco han de hacer para sacar lo mejor de ti. Por haber aguantado mi verborrea durante estos tres años y seguir apreciándome pese a ello, os doy las gracias.

También quiero agradecer el haber tenido un buen entorno de trabajo en la UPV, rodeada de unos compañeros siempre dispuestos a ayudar y a hacer el día a día más ameno. En especial, mi compañero Ricardo Belda ha sido mi mayor cómplice durante esta cruzada y un apoyo en los momentos más difíciles. Por supuesto, este trabajo no habría sido posible sin la impecable dirección de Eugenio Giner, con quien he aprendido no sólo ingeniería mecánica, sino 
también el arte de hacer las cosas con sentido.

En allant un peu plus au nord, je voulais remercier Rèmy Willinger et Caroline Deck de me donner l'occasion d'enquêter sur leurs installations et de faire connaissance avec la merveilleuse ville de Strasbourg, dont je garderai toujours un beau souvenir.

Dejando lo mejor para el final, quiero dar las gracias a Nacho Serrano, mi amigo y compañero de vida, que tanto me ha ayudado en confiar en mis habilidades y que me ha dado fuerzas para terminar esta Tesis con ilusión y mirar el futuro con optimismo. Las cosas parecen mucho mejores a tu lado. 


\section{Abstract}

Impact loading is the primary source of head injuries and can result in a range of trauma from mild to severe. Because of the multiple environments in which impact-related injuries can take place (automotive accidents, sports, accidental falls, violence), they can potentially affect the entire population regardless of their health conditions. Despite the increasing research effort on the understanding of head impact biomechanics, accurate prediction and prevention of traumatic injuries has not been completely achieved.

In this Thesis, some aspects of the impact behaviour of the different biological tissues involved have been analysed through the development of a numerical human head model from Computed Tomography (CT) images. FE simulations of experimental tests from the literature have been performed and enhanced the validation of the head model through the establishment of proper material laws for its constituents, which enable adequate prediction of injury risks. Part of this Thesis focuses on the ballistic environment, especifically in bulletproof composite helmets, which are susceptible to cause blunt injuries to the head because of their large deformation during impact. Prior to the study of these high-speed impacts, experimental tests and finite element (FE) models have been performed to characterise the mechanical response of composite materials subjected to low velocity impact. The implementation of a continuum damage mechanics approach coupled to a Hashin failure criterion and surface-to-surface cohesive relations to the numerical model provided a good matching with the impact behaviour obtained experimentally, capturing the principal damage mechanisms. A review of the head injury criteria currently available in the literature has been performed at the beginning of this Thesis. This is a key issue for the numerical simulations, as the suitability of some criteria to predict head injuries is still an open question.

Numerical simulation of ballistic impacts on a human head protected with 
a combat helmet has been conducted employing explicit FE analysis. The level of protection ensured by helmet acceptance protocols as well as their sizing strategies have been studied and discussed by means of the application of different mechanical-based head injury criteria. It has been demonstrated that current helmet testing standards do mitigate some specific forms of head trauma but fail to prevent other injury mechanisms such as the intracranial pressure gradients within the skull. Furthermore, it has been demonstrated that some well-established helmet sizing policies like manufacturing one single composite shell and adapting the thickness of the interior pads to the different head dimensions should be reconsidered, as there is a great risk of head injury when the distance between the head and the helmet shell (stand-off distance) is not sufficient.

Following the line of personal protections, some composite materials commonly employed in the soft body armour industry have been combined into different helmet shells configurations to optimise the ratio of weight-to-head protection. Light materials like UHMWPE appear to be less efficient than integral woven-aramid lay-ups in the limitation of the backface deformation (BFD), the maximum deformation sustained by the helmet at the impact site.

A detailed head numerical model including thirty-three of its main anatomical structures has been developed for the simulation of an equestrian accident that resulted in many head injuries. Above all, the establishment of a mechanical criterion for the prediction of subdural hematona (SDH) based on the rupture of the head blood vessels is intended. A stress threshold for vein rupture has been set on $3.5 \mathrm{MPa}$, but both this limit and the location of vessel failure are highly dependent on the specific anatomy of the subject's vascularity. 


\section{Resumen}

Las cargas de impacto son la fuente primaria de lesiones en la cabeza y pueden resultar en un rango de traumatismo desde leve hasta severo. Debido a la existencia de múltiples entornos en los que se pueden desencadenar lesiones por impacto (accidentes automovilísticos, deportes, caídas accidentales, violencia), éstas pueden afectar potencialmente a toda la población independientemente de su estado de salud. Pese al creciente esfuerzo en investigación para comprender la biomecánica de las lesiones por traumatismo en la cabeza, todavía no es del todo posible realizar predicciones precisas ni prevenir estos eventos.

En esta Tesis, se han estudiado algunos aspectos del comportamiento ante impacto de los diferentes tejidos biológicos involucrados mediante el desarrollo de un modelo numérico de cabeza humana a partir de imágenes de tomografía computerizada (TAC). Se han realizado simulaciones en elementos finitos (EF) de ensayos experimentales de la literatura con el fin de validar el modelo numérico desarrollado, estableciendo unas propiedades mecánicas adecuadas para cada uno de sus constituyentes. De esta manera se puede adquirir una predicción adecuada del riesgo de sufrir daños. Parte de esta Tesis se centra en el entorno balístico, específicamente en cascos de combate antibalas, los cuales son susceptibles de causar traumatismo craneoencefálico debido a la elevada deformación que sufren durante el impacto. Previamente al estudio de estos fenómenos de alta velocidad, se han realizado ensayos experimentales y numéricos para caracterizar la respuesta mecánica de algunos materiales compuestos ante impacto de baja velocidad. Se ha obtenido una adecuada caracterización de los mecanismos de daño y fallo de estos materiales mediante la implementación de un modelo de mecánica de daño continuo junto con un criterio de fallo de Hashin y relaciones cohesivas de tipo superficie-superficie. Al principio de esta Tesis se ha realizado una revisión del estado del arte acerca de los criterios existentes para cuantificar el trauma craneoencefálico. 
Este es un aspecto clave para las simulaciones numéricas, ya que la idoneidad de algunos de estos criterios para la predicción de lesiones cerebrales todavía es un debate abierto.

Mediante EF se han realizado simulaciones numéricas de impactos balísticos en una cabeza protegida con un casco de combate. Mediante la posterior aplicación de diferentes criterios de daño sobre los resultados obtenidos se ha evaluado el nivel de protección que aseguran los protocolos de aceptación de cascos de combate, así como las estrategias para determinar su tallaje. Se ha demostrado que las normativas existentes para cascos de combate son capaces de mitigar algunos mecanismos de trauma pero no logran prevenir otros como los gradientes de presión intracraneales. Además, se ha demostrado que algunas de las estrategias de tallaje más comúnmente adoptadas por los fabricantes, como producir un solo tamaño de calota y adaptar el espesor de las espumas interiores a las diferentes dimensiones de los sujetos, deberían ser reconsideradas ya que existe un mayor riesgo de traumatismo cuando la distancia entre la cabeza y la calota del casco no es suficiente.

Siguiendo la línea de protecciones personales, algunos de los materiales compuestos comúnmente empleados en la industria armamentística se han combinado para crear distintas posibles configuraciones de calota para optimizar la relación entre peso del casco y protección para la cabeza. Materiales ligeros como el UHMWPE han resultado en un comportamiento menos eficiente que el de los apilados de tejido de aramida a la hora de limitar la BFD (deformación máxima en la calota del casco en la zona de impacto).

Hacia el final de la Tesis se presenta un modelo numérico de cabeza humana detallado, que incluye treinta y tres de las estructuras anatómicas principales. Dicho modelo se ha desarrollado para la simulación de un accidente ecuestre en el que aparecen múltiples lesiones craneoencefálicas. Principalmente, se pretende establecer un criterio mecánico para la predicción del hematoma subdural (HS) basado en la ruptura de los vasos sanguíneos intracraneales. Se ha propuesto un valor umbral de ruptura en tensiones de $3.5 \mathrm{MPa}$, pero tanto este límite como la localización del vaso dañado son altamente dependientes de la anatomía específica de cada sujeto. 


\section{Resum}

Les càrregues d'impacte son la font primària de lesions al cap i poden resultar en un rang de severitat des de lleu a greu. Degut als múltiples entorns en que poden desencadenar-se lesions per impacte (accidents automobilístics, esports, caigudes accidentals, violència), aquestes poden afectar potencialment a tota la població independentment del seu estat de salut. Malgrat el creixent esforç en investigació per comprendre la biomecànica de les lesions per traumatisme al cap, encara no és del tot possible realitzar prediccions precises ni prevenir aquestos esdeveniments.

En aquesta Tesi, s'han estudiat alguns aspectes del comportament a impacte dels diferents teixits biològics involucrats mitjançant el desenvolupament d'un model numèric de cap humà a partir d'imatges de tomografia computeritzada (TAC). S'han realitzat simulacions en elements finits (EF) d'assajos experimentals de la literatura amb la finalitat de validar el model numèric desenvolupat, establint unes propietats mecàniques adequades per a cadascun dels seus constituents. D'aquesta manera es pot aconseguir una predicció del risc de sofrir danys traumàtics. Part d'aquesta Tesi es centra en l'entorn balístic, específicament en cascs de combat antibales, els quals són susceptibles de causar traumatisme degut a l'elevada deformació que sofrixen durant l'impacte. Previament a l'estudi d'aquests fenòmens d'alta velocitat, s'han realitzat assajos experimentals i numèrics per a caracteritzar la resposta mecànica d'alguns materials compostos en condicions d'impacte a baixa velocitat. S'ha obtingut una adequada caracterització dels mecanismes de dany i fallada d'aquestos materials mitjançant la implementació d'un model de mecànica de dany continu amb un criteri de fallada de Hashin i relacions cohesives de tipus superfíciesuperfície. Al començament d'aquesta Tesi s'ha realitzat una revisió de l'estat de l'art sobre els criteris existents per quantificar el trauma cranioencefàlic. Aquest és un aspecte clau per a les simulacions numèriques, ja que l'utilitat 
d'alguns d'aquestos criteris per a la predicció de lesions cerebrals és encara un debat obert.

Mitjançant EF s'han realitzat simulacions numèriques d'impactes balístics en un cap protegit amb un casc de combat. Gràcies a la posterior aplicació de diferents criteris de dany sobre els resultats obtinguts s'ha evaluat el nivell de protecció que asseguren els protocols d'acceptació de cascs de combat, així com les estratègies per a determinar les seues talles. S'ha demostrat que les normatives existents són capaces de mitigar alguns mecanismes de trauma però no aconseguixen prevenir altres com els gradients de pressions intracranials. A més, s'ha demostrat que algunes estratègies per determinar les talles més comunament adoptades pels fabricants (com produir només un tamany de calota i adaptar el gruix de les escumes interiors a les diferents dimensions dels subjectes) haurien de ser reconsiderades ja que existeix un major risc de traumatisme quan la distància entre el cap i la calota del casc no és suficient.

Seguint la línia de proteccions personals, alguns dels materials compostos comunament utilitzats en la indústria de l'armament s'han combinat per a crear distintes possibles configuracions de calota amb la finalitat d'optimitzar la relació entre pes i protecció. Materials lleugers com l'UHMWPE han resultat en un comportament menys eficient que el d'apilats de teixit d'aramida a l'hora de limitar la BFD (deformació màxima a la calota del casc a la zona d'impacte).

Cap al final de la Tesi es presenta un model numèric detallat de cap humà, que inclou trenta-tres de les estructures anatòmiques principals. Aquest model s'ha desenvolupat per a la simulació d'un accident eqüestre en el qual apareixen múltiples lesions cranioencefàliques. Principalment, es pretén establir un criteri mecànic per a la predicció de l'hematoma subdural (HS) basat en la ruptura dels vasos sanguinis intracranials. S'ha proposat un valor umbral de ruptura en tensions de 3.5 MPa, pero tant aquest límit com la ubicació del vas danyat són altament dependents de l'anatomia específica de cada subjecte. 



\section{Acronyms}

$\mathrm{ACH}$

$\mathrm{ASDH}$

ATD

BFD

BHBT

$\mathrm{BLSH}$

BPT

$\mathrm{BSF}$

BV

CAI

$\mathrm{CDM}$

CFRP

CNS

CSDM

$\mathrm{CSF}$

$\mathrm{CT}$

CZM

DAI

DDM

DIC

DST

DTI

$\mathrm{ECH}$

$\mathrm{EDH}$

EOS

FE

FEA
Advanced Combat Helmet

Acute Subdural Hematoma

Anthropomorphic Test Device

Backface deformation

Behind Helmet Blunt Trauma

Ballistic Load Sensing Headform

Brain Pressure Tolerance Basilar Skull Fracture Bridging Vein

Compression After Impact Continuum Damage Mechanics Carbon Fiber-Reinforced Plastic

Central Nervous System Cumulative Strain Damage Measure Cerebrospinal Fluid Computed Tomography Cohesive Zone Models Diffuse Axonal Injury Dilatation Damage Measure Digital Image Correlation Dynamic Shear Testing Diffusion Tensor Imaging Enhanced Combat Helmet Epidural Hematoma Equation of State Finite Element Finite Element Analysis 
FEM

FMJ

HIC

HIP

$\mathrm{HU}$

ICP

IE

LWH

MPS

MRI

MRI

MSC

NDT

NIJ

NLI

NLP

NLW

PASGT

PMHS

RMDM

ROI

SAH

$\mathrm{SDH}$

SFC

SPR

SSS

TBI

TEM

UHMWPE

WF

WSTC
Finite Element Method

Full Metal Jacket Head Injury Criterion Head Impact Power Hysteretic Unloading Intracranial Pressure Internal Energy Lightweight Combat Helmet Maximum Principal Stress Magnetic Resonance Elastography Magnetic Resonance Imaging Mean Strain Criterion Neutral Density Target National Institute of Justice Non-linear Inversion Non-lethal Projectile Non-lethal Weapon Personnel Armour System Ground Troops Postmortem Human Subjects Relative Motion Damage Measure Region of Interest Subarachnoid Haemorrhage Subdural hematoma Skull Fracture Correlate Soft Prior Regularization Superior Sagittal Sinus Traumatic Brain Injury Transmission Electron Microscopy Ultra-high Molecular Weight Polyethylene Woven Fabric Wayne State Tolerance Curve 



\section{Contents}

Agradecimientos $\quad$ i

Abstract

Resumen $\quad$ V

Resum vii

Acronyms $\quad$ x

1 Introduction $\quad 1$

1.1 Motivation and aims .................. . . 1

1.2 Organisation of the Thesis .............. . 2

2 Head biomechanics $\quad \mathbf{5}$

2.1 Mechanical behaviour of the skull . . . . . . . . . . . . . 6

2.1.1 Studies on bone samples . . . . . . . . . . . . . 7

2.1.2 Studies on whole cranium . . . . . . . . . . . 12

2.2 Brain tissue biomechanics . . . . . . . . . . . . . . 16

2.2.1 Experimental approaches . . . . . . . . . . 18

2.2.2 Constitutive modeling of brain tissue . . . . . . . . . 31

2.3 Other structures of the head . . . . . . . . . . . . . 39

2.3.1 The meninges ................. 39

2.3.2 Falx and tentorium ............... . 41

2.3.3 Blood vessels .................. . 42

2.3.4 Scalp .................... 42

2.4 Head Injury Criteria . . . . . . . . . . . . . . . . 43

2.4.1 Introduction to head injuries . . . . . . . . . . 43 
2.4.2 Head injury criteria . . . . . . . . . . 46

3 Explicit Finite Element Analysis $\quad 59$

3.1 Equations of transient dynamics . . . . . . . . . . . . 59

3.1 .1 Discretisation in space . . . . . . . . . . . . 62

3.2 Time dependency: implicit and explicit FEA . . . . . . . 62

3.2 .1 Implicit schemes . . . . . . . . . . . . . . . . . 63

3.3 The explicit scheme . . . . . . . . . . . . . 63

3.4 Conditional stability . . . . . . . . . . . . . . 65

3.5 Viscous damping . . . . . . . . . . . . . . . . . . . 69

3.6 Diagonal mass matrix . . . . . . . . . . . . . . 70

3.7 Element controls . . . . . . . . . . . . . . . . . . 70

3.8 Contact in explicit FEA . . . . . . . . . . . . . 73

3.8.1 The Lagrange multipliers method . . . . . . . . . . . . . 74

3.8 .2 The penalty method . . . . . . . . . . . 76

4 Impact behaviour of protective materials $\quad 79$

4.1 Overview . . . . . . . . . . . . . . . 80

4.2 Experimental tests on composite plates . . . . . . . . . . . 81

4.2.1 Experimental results . . . . . . . . . . . . . . . 83

4.3 High-speed camera measurement . . . . . . . . . . . . . 86

4.3.1 Digital Image Correlation (DIC) . . . . . . . . . . 88

4.4 FE explicit simulation . . . . . . . . . . . . . . . . . . . 93

4.4 .1 Model description . . . . . . . . . . . . . . . . . 93

4.4.2 Interlaminar damage using CZM . . . . . . . . . . . 97

4.4 .3 Results . . . . . . . . . . . . . . . . 100

4.5 Accuracy of the methodology . . . . . . . . . . 108

5 Numerical modeling of a human head 111

5.1 Head FE models in the literature . . . . . . . . . . . . . . . 111

5.2 Model development . . . . . . . . . . . . . . . . . 113

5.2 .1 CT image segmentation . . . . . . . . . . . . 113

5.2 .2 Mesh . . . . . . . . . . . . . . . . 116

5.3 Material modeling . . . . . . . . . . . . . . . . . 118

5.4 Calibration of the model . . . . . . . . . . . . . . 120

5.4.1 Dynamic mechanical properties of the cranium and scalp 121

5.4 .2 Brain tissue calibration . . . . . . . . . . . . 130

5.5 Validation against high velocity impact conditions . . . . . 136 
5.6 Model limitations . . . . . . . . . . . . . . . . . . . . . . 140

6 Head and helmet coupling under ballistic testing 143

6.1 Helmet model . . . . . . . . . . . . . . . . . . . . . . . . 145

6.1.1 Helmet composite shell . . . . . . . . . . . . . 145

6.1.2 Interior padding system . . . . . . . . . . . . . 147

6.2 Ammunition model . . . . . . . . . . . . . . . . . . . . . 148

6.3 Coupling and simulation conditions . . . . . . . . . . 151

6.4 Study of the NIJ Standard (NIJ-STD-0106.01) . . . . . . . . 153

6.5 Effect of projectile initial velocity . . . . . . . . . . . 158

6.5.1 Performance of the helmet shell . . . . . . . . . . . . 159

6.5.2 Assessment of head trauma . . . . . . . . . . . . 162

6.6 Effect of helmet size . . . . . . . . . . . . . . 166

7 Effect of stack configuration of the helmet shell $\quad 171$

7.1 Shell configurations . . . . . . . . . . . . . . . 173

7.2 Material modeling . . . . . . . . . . . . . . . . 176

7.3 Comparison of the protective capacity . . . . . . . . 179

7.3.1 Aramid configurations . . . . . . . . . . . . . . 179

7.3.2 UHMWPE configurations . . . . . . . . . . . . . 183

7.3.3 Hybrid configurations: Aramid and Carbon Fiber . . . . 185

7.3.4 Hybrid configurations: UHWMPE and aramid . . . . . 189

7.4 Associated head trauma . . . . . . . . . . . . . . . . 193

7.4.1 Conclusions . . . . . . . . . . . . . . . . . 194

8 Numerical modeling of a human head with further anatomical $\begin{array}{ll}\text { details } & 197\end{array}$

8.1 Model description . . . . . . . . . . . . . . . . . . . 198

8.2 Validation . . . . . . . . . . . . . . . . . 203

8.3 Application to an accident reconstruction . . . . . . . . . . 211

8.4 Prediction of subdural hematoma . . . . . . . . . . . . . 213

8.4.1 Inclusion of the bridging veins . . . . . . . . . 215

8.5 Towards an injury criterion . . . . . . . . . . . . 222

9 Characterisation of Non-Lethal Projectiles 225

9.1 Background . . . . . . . . . . . . . . . . 225

9.2 Methodology . . . . . . . . . . . . . . . . 227

9.3 Experimental data treatment . . . . . . . . . . . . . 228 
9.3.1 Video edition . . . . . . . . . . . . . . . . . . 229

9.3.2 Sensor data extraction . . . . . . . . . . . . 229

9.3.3 Point tracking . . . . . . . . . . . . . . 230

9.3.4 Mechanical response of the projectile . . . . . . . . 233

9.3.5 Results and statistics . . . . . . . . . . . . . . 234

9.4 Numerical modeling . . . . . . . . . . . . . . . . . . . . 235

9.5 Results . . . . . . . . . . . . . . . . . . . . 237

9.6 Conclusions . . . . . . . . . . . . . . . . . . . 242

10 Conclusions and future works $\quad 245$ 


\section{List of Figures}

2.1 Human skull bones. . . . . . . . . . . . . . . . . 6

2.2 The three different bone layers of the cranium: compact bone tables and diplöe core. . . . . . . . . . . . . 7

2.3 Neural tissue and structures of the brain. . . . . . . . . . 17

2.4 Typical stress relaxation curves for brain tissue in compression (left) and shear (right). Experimental curves extracted from the tests conducted by Budday et al. [45] for different brain regions. $\mathrm{C}=$ cortex, $\mathrm{CR}=$ corona radiata, $\mathrm{BG}=$ basal ganglia, $\mathrm{CC}=$

2.5 Four-element rheological models proposed by Galford and . . . . . . . . . . . . haney [30] (a) and Shuck and Advani [32] (b). Three-element solid (c) and four-element fluid (d) from Pamidi and Advani [69]. 32

2.6 One-parameter Ogden models fitting the experimental curves from Budday et al. [71] for different brain regions. $\mathrm{C}=$ cortex, $\mathrm{CR}=$ corona radiata, $\mathrm{BG}=$ basal ganglia, $\mathrm{CC}=$ corpus callosum. 35

2.7 Scheme of the non-linear visco-hyperelastic material model proposed by Prevost et al. [46]. . . . . . . . . . . . . . . . 36

2.8 Scheme of the meninges membranous complex. . . . . . . . . 40

2.9 Classical classification of head injuries . . . . . . . . . . 45

2.10 Wayne State Tolerance curve. . . . . . . . . . . . . . 46

3.1 Rod element with an initial velocity. . . . . . . . . . . . 66

3.2 Explicit solution of the rod problem using different time step values and analytical solution of the problem. . . . . . . . 68

3.3 Different hourglass modes on 3D solid elements. . . . . . . . . . 71

4.1 Experimental setup for blunt impact tests on composite samples. 82 
4.2 External (a) and striking surface (b) of a plate tested at an impact energy of $29 \mathrm{~J}$. Superficial damage at the exterior (c) and interior layers $(\mathrm{d}) \ldots \ldots \ldots \ldots \ldots$

4.3 External (a) and striking surface (b) of a plate tested at an impact energy of $68.10 \mathrm{~J}$. (c) Detail of the delamination at the free edge.

4.4 Time sequence of crack growth during impact recorded with high-speed camera at a frame rate of $8000 \mathrm{fps}$.

4.5 Variation of the step and subset values and its influence on DIC using Vic 2D

4.6 Output displacement fields employing three different correlation criteria in Vic 2D. . . . . . . . . . . . . . . . . . 93

4.7 Abaqus/Explicit setup of the impact tests. . . . . . . . . . . 94

4.8 Diagram of an explicit procedure including a VUMAT subroutine for damage evolution. Extracted from Wang et al. (2014) $[183] \ldots \ldots \ldots \ldots \ldots \ldots \ldots \ldots$

4.9 Graphical representation of the mixed-mode cohesive interaction implemented in Abaqus/Explicit. . . . . . . . . . . . . 99

4.10 Absorbed energy $E_{a b s}$-time and force-displacement curves for the simulated impacts on the CFRP plate.

4.11 Simulated interlaminar mixed-mode failure in the composite plates. Red-colored regions indicate that damage between plies is initiated. The illustrated sequence corresponds to the $34 \mathrm{~J}$ case.

4.12 Experimental damage at the composite plate at the striking and non-striking surfaces and comparison with numerical simulations at different energy levels. . . . . . . . . . . . 106

4.13 Comparison of the strain fields obtained by means of FE simulation and DIC for an impact energy level of $23 \mathrm{~J}$ (top) and 68 $\mathrm{J}$ (top). The variable represented is the shear deformation $\varepsilon_{x y}$. Due to symmetry, only the left half of the plate is represented.

5.1 CT images of the head of a human subject in different views: sagittal (top left), horizontal (top right) and coronal (bottom left). Three-dimensional rendered reconstruction performed with ScanIp (bottom right). . . . . . . . . . . . . . . . . . 114

5.2 Resulting finite element mesh from CT image segmentation. The six principal tissues included in the model are illustrated. . 117 
5.3 Reconstruction in Abaqus/Explicit of the experimental impact test by Yoganandan et al. [21]. . . . . . . . . . . . . . . . 122

5.4 Calibration on the ultimate strain for the scalp tissue based on the experiment by Yoganandan et al. [21] . . . . . . . . . . . . 123

5.5 Force-displacement response of the in-house numerical head model with different failure criteria for the cranial bone layers. . . . . 125

5.6 Force-deflection response of the head model with each set of mechanical properties against the experimental data by Yoganandan et al. [21].

5.7 Pressure transducers location in the experiment by Nahum et al. [191]: frontal (1), parietal (2), occipital (3) and posterior fossa(4) . . . . . . . . . . . . . . . 131

5.8 Abaqus/Explicit setup of experiment 37 by Nahum et al. [191]. 131

5.9 Comparison of the force and acceleration histories between the numerical simulation and the experimental curves reported by Nahum et al. [191]. . . . . . . . . . . . . . . . . . . . . . . 132

5.10 Intracranial pressures at different brain locations obtained with numerical simulations and the experimental curves from Nahum et al. [191]. . . . . . . . . . . . . . . . . . . 134

5.11 Effect on intracranial pressure of the variation of the bulk modulus implemented in MP3 under the impact conditions [191]. . 135

5.12 Initial and final states in the Abaqus/Explicit simulation of the experimental ballistic tests (number \#27, \#68 and \#72) from Sarron et al. [222] on human cadaveric heads. . . . . . . . . . . 139

6.1 Four-layered numerical model of the helmet shell employed in this study. . . . . . . . . . . . . . . . . . . 146

6.2 Mesh of the modeled foam padding system (left) and its adhesion to the helmet shell (right). . . . . . . . . . . . . . . 148

6.3 Real $9 \mathrm{~mm}$ caliber FMJ bullet (left), mesh for the numerical bullet model including its two parts (middle) and deformed shape after a high-speed impact (right). . . . . . . . . . . . . . . . 149

6.4 Low-velocity insertion of the combat helmet in the head model in Abaqus/Explicit (left), incorporation of the bullet model and final coupled system (right) . . . . . . . . . . . . . . . . . 152 
6.5 Ballistic headform employed for the testing protocol of the NIJ standard. The location of the five compulsory shots with FMJ bullets is schematised in the top view of the headform (bottom right of the figure). . . . . . . . . . . . . . 154

6.6 Transversal cut in the simulation at the time of maximum BFD for each of the impact sites stated by the standard: a) frontal, b) lateral, c) occipital, d) vertex. . . . . . . . . . . . . 156

6.7 Evolution of the maximum BFD registered in the numerical helmet model at different projectile velocities under frontal impact conditions. . . . . . . . . . . . . . . . . . 160

6.8 Evolution of the maximum BFD registered in the numerical helmet model at different projectile velocities under lateral (temporoparietal) impact conditions.

6.9 Values of the skull internal energy obtained for each simulation at a given initial projectile velocity for frontal (left) and lateral (right) ballistic impacts. . . . . . . . . . . . . . .

6.10 Time evolution of a linear skull fracture at the frontal head site from an impact velocity of $595 \mathrm{~ms}^{-1}$ (top) and stellate fracture caused by an impact at $615 \mathrm{~ms}^{-1}$ (bottom) . . . . . . . . . 163

6.11 Linear cracks at the inner surface of the cranial vault at the temporoparietal region resulting from an impact at $540 \mathrm{~ms}^{-1}$ (top) and depressed skull fracture at the impact site for a velocity of $545 \mathrm{~ms}^{-1}$

6.12 M size helmet coupled to a $56 \mathrm{~cm}$ circumference head model (top) and L size helmet protecting a head model whose circumference is $61 \mathrm{~cm}$.

6.13 Evolution of the internal energy of the skull model for different impact velocities in a frontal shot using an L-size protection. Maximum BFD configurations are represented at the right side. 168

6.14 Mild (top) and severe (bottom) frontobasal skull fractures caused by frontal ballistic impacts on an L-size helmet configuration at 523 and $530 \mathrm{~ms}^{-1}$, respectively. . . . . . . . . . . 170

7.1 Left: helicoidal-pattern lay-up of unidirectional plies for configuration \#P1. Right: scheme of the cross-ply structure of the HB26 stack employed in the modeling of \#P2, \#P3 and \#P4. 
7.2 Detailed view of the hybrid configurations tested in this study (\#HCA, \#HAC, \#HAP, \#HPA). The reinforcing material is localised whether in the striking or the inner surface. . . . . . . 175

7.3 Maximum backface deformation and energy absorbed by the two aramid-fabric configurations.

7.4 First row: displacement contours of the inner impacted region of the helmet shell for \#A1 (left) and \#A2 (right). Second row: cross-section of the deformed shell for \#A1 (left) and \#A2 (right).

7.5 Frontal view of the failed elements after the impact. The most clear-cut elements correspond to the exterior surface, while increased translucency means location at the deeper layers. . . . 181

7.6 Backface deformation and energy absorbed by each of the UHMWPEbased helmet shells. . . . . . . . . . . . . . . . . 183

7.7 Time sequence of the $9 \mathrm{~mm}$ bullet impacting the \#P1 shell. . 184

7.8 Deformation of shells \#P2, \#P3 and \#P4 at the region impacted by the bullet.

7.9 Backface deformation and energy absorbed by each of the carbonaramid hybrid shells and the aramid-only configuration \#A1.

7.10 Transversal cut of the helmet shell at two different times from the impact simulations on shells \#HAC (top) and \#HCA (bottom).

7.11 Maximum backface deformation and energy absorbed by the hybrid UHMWPE-aramid configurations and the UHMWPEonly shell.

7.12 Cross section of the deformed shells \#HAP (left) and \#HPA (right).

7.13 Delamination during impact in the \#HAP (left) and \#HPA (right) shells.

8.1 Some of the intracranial structures included in the FE head model presented in this Chapter. . . . . . . . . . . . . . . . 200

8.2 Other constituents of the current FE head model. Top: cerebrum divided into white and gray matter. Bottom: bony structures, dura matter and CSF layer. . . . . . . . . . . . . . 201

8.3 Scheme of the experimental test C755-T2 from [256], corresponding to an occipital blow. The NDTs where relative displacement is measured are depicted in red colour. 
8.4 Cut in the head's coronal plane with the scheme of the experimental test C383-T1 from [257], corresponding to left parietal blow. The NDTs are clustered in groups of 7 (red dots). . . . 205

8.5 Boundary conditions applied to the six degrees of freedom of the head model for experiments C755-T2(top) and C383-T1(bottom) from [256]. . . . . . . . . . . . . . . . . . . 206

8.6 Relative brain-skull displacement obtained from the FE simulation of test C755-T2 from [256]. . . . . . . . . . . . . . . . 207

8.7 Brain motion subsequent to the lateral impact test C383-T1 from [256]. . . . . . . . . . . . . . . . . . . . . . . . 208

8.8 Simulated and experimental relative displacements in the $\mathrm{X}$ and $\mathrm{Z}$ directions for the NDTs reported in test C755-T2 from [256]. 209

8.9 Simulated and experimental relative displacements in the $\mathrm{Y}$ and $\mathrm{Z}$ directions for the NDTs reported in test C380-T1 from [256].

8.10 Reconstruction of the cranial fractures from the CT images with ScanIP. ...................... 211

8.11 Imaging of the intracranial lesions after impact. . . . . . . . 212

8.12 Cranial fracture obtained through numerical simulation matching the patterns observed in the original CT scan of the patient. 213

8.13 Rendered view of the top skullcap from the CT scan. Intense red areas indicate blood accumulation representing the $\mathrm{SDH}$ and SAH. . . . . . . . . . . . . . . . . . . . . . . . . 214

8.14 Anatomical distribution of the bridging veins in a real head (left) and their simplified architecture for the numerical model of this section (right). . . . . . . . . . . . . . . 216

8.15 Maximum relative displacement between the brain and the SSS from the baseline model (left) and the model including the BV (right).

9.1 Methodology followed for the validation of a numerical NLP model ..................... . . 228

9.2 Cut view of the point tracking tab after the computation of the contact diameter (red) between the projectile and the wall.

9.3 Automatic detection of the contact diameter (red) between the projectile and the wall. . . . . . . . . . . . . . . . 233

9.4 Structure of the data stored from each experimental test in the Mechanical response tab. . . . . . . . . . . . . . . 234

9.5 Projectile hexahedral mesh obtained by means of Hypermesh. . 236 
9.6 LS-DYNA assembly for the simulation of impact against rigid wall. . . . . . . . . . . . . . . . . . . 237

9.7 Impact force measured at the rigid wall for the three initial kinetic energies tested. Mean experimental curves in solid black line, upper and lower experimental corridors in dashed line, and numerical response in red. . . . . . . . . . . . . . . . . 238

9.8 Comparison between the simulated and experimental displacements at the rear of the projectile. . . . . . . . . . . . 239

9.9 Maximum diameter of the projectile during the impact event calculated experimentally and numerically. . . . . . . . . . . . 240

9.10 Influence on the unloading parameters on the maximum diameter and bottom displacement of the projectile. . . . . . . . . 241 



\section{List of Tables}

2.1 Summary of published works on skull bone samples . . . . . . . 11

2.2 Summary of published results on skull fracture load. . . . . . . 16

2.3 Some reported elastic properties of the cerebrum and its two main constituents from the literature. $\mu$ refers to the shear stiffness, $G$ to the shear modulus and $G^{\prime}, G^{\prime \prime}$ represent the storage and loss moduli, respectively. . . . . . . . . . . .

2.4 Experimentally obtained elastic properties from other brain regions. $\mu$ refers to the shear stiffness, $G$ to the shear modulus and $G^{\prime}, G^{\prime \prime}$ represent the storage and loss moduli, respectively. . 30

2.5 Summary of some published head injury criteria. . . . . . . . 58

4.1 Experimental test matrix for the pendulum blunt impact on CFRP plates. . . . . . . . . . . . . 83

4.2 Summary of the experimental results from the impact tests. . . 85

4.3 Mechanical parameters set to model the cohesive interaction between the composite plies. . . . . . . . . . . . . 99

4.4 Material properties applied to the woven CFRP plies of the plate in the numerical model. . . . . . . . . . . 100

5.1 Summary of the mechanical properties of the different head tissues of the five models extracted from the literature. . . . . . 119

5.2 Ultimate strength values for the cranial bone layers reported in different works from the literature. . . . . . . . . . . 125

5.3 Computed skull stiffness values from each simulation and the experimental value measured by Yoganandan et al.[21] . . . . 129

5.4 Mechanical properties chosen for the scalp and cranial bones of the numerical head model. . . . . . . . . . . . . . . . 130 
5.5 Hyper-viscoelastic properties of brain tissue. . . . . . . . . . . 136

5.6 Test conditions for the simulated experiments from Sarron et al. [222] and predicted intracranial (cisternal) pressures after impact by the numerical model from this Thesis. . . . . . . . . 140

6.1 Summary of the mechanical properties of the aramid fabric layers conforming the helmet shell. . . . . . . . . . . . . . 146

6.2 Material constants for the two components of the bullet model. 151

6.3 Obtained values for certain head injury metrics under the simulated ballistic impacts at the locations stated by the NIJ-STD0106.01. Last column presents critical threshold values taken from the literature. . . . . . . . . . . . . . . . . 155

6.4 Comparison of the injury metrics computed from the FE simulation of the NIJ testing protocol for a medium and a large size configuration. . . . . . . . . . . . . 168

7.1 Different helmet shell configurations tested in this work. . . . . 174

7.2 Material properties for the woven CFRP plies of the plate. . . . 176

7.3 Summary of the mechanical properties of the unidirectional UHMWPE plies from Yang and Chen [252] . . . . . . . . . 177

7.4 Summary of the mechanical properties of the UHMWPE composite extracted from the work by Nguyen et al [253]. . . . . . 178

7.5 Head injury outcome from the different helmet shell configurations simulated. . . . . . . . . . . . . . . 193

8.1 Mechanical properties of the head tissues assumed as linear elastic in the numerical model. . . . . . . . . . . . . . . . . 202

8.2 Mechanical properties of the different constituents of the brain applied to the head model. . . . . . . . . . . . . . . . 203

8.3 Predicted principal strains on the bridging veins complex during impact loading $(\mathrm{t}=4.5 \mathrm{~ms})$ in the baseline model and the model with $\mathrm{BV} . \mathrm{FP}=$ frontopolar, $\mathrm{AF}=$ anterior frontal, $\mathrm{MF}=$ middle frontal, $\mathrm{PF}=$ posterior frontal, $\mathrm{PC}=$ precentral, $\mathrm{VT}=$ vein of Trolard, $\mathrm{C}=$ central, $\mathrm{PsC}=$ postcentral, $\mathrm{AP}=$ anterior parietal, $\mathrm{PP}=$ posterior parietal, $\mathrm{O}=$ occipital. $\ldots \ldots .218$

8.4 Kinematic boundary conditions from the tests performed by Depreitere et al. [137] simulated in this section. . . . . . . . . . 219 
8.5 Predicted strains on the bridging veins complex during impact loading $(\mathrm{t}=4.5 \mathrm{~ms})$ in the model with $\mathrm{BV}$ in the cases with and without skull fracture. . . . . . . . . . . . . . 221 


\section{Chapter 1}

\section{Introduction}

\subsection{Motivation and aims}

Head injury is a high-magnitude social and economic issue due to its increasing affection to both civilian and military population being nowadays one of the principal causes of death in the automotive, sportive and accidental environments. Consequences of head trauma range from mild to fatal and there is a necessity to understand its triggering mechanisms in order to design safer protection mechanisms and treatment protocols.

This milestone is only reachable by means of extensive research in the biomechanical field. Proper characterisation of the living tissues that constitute the human head is the first step for predicting and understanding its behaviour when subjected to different loading conditions. Nonetheless, the complexity of the problem is huge due to the difficulty of performing in vivo tests, the great intra and interspecimen variability and the different response of the living tissues depending on the impact scenario. On the other hand, finite element (FE) modeling appears as a versatile tool offering multiple simulation possibilities at a reasonable time cost and reduced economic expense. Even though there have been substantial breakthroughs in the field of head injury since the middle nineteenth century, many aspects of the biomechanics of trauma remain unkown mainly due to the lack of consensus in both the experimental and numerical data.

Concerning head trauma biomechanics, one aspect that has been scarcely studied in the literature is the injury prediction under high-speed impacts, 
specifically in the ballistic range. Personal protections aim to mitigate the magnitude of impact-induced loads on the human body and many of them (i.e. helmets for cyclists, motorcyclists, contact sports) have adapted their design according to the new discoveries achieved in research in order to increase their safety level. In fact, standards for some helmet typologies are periodically reformulated or modified to limit their performance to a noninjurious envelope. However, the improvements in risk prevention arise slower in the field of ballistic protections. Indeed, most of the existing regulations for ballistic helmets are derived from the premises of the automotive or sportive environment, even though their characteristics are substantially different.

Inside this context, the purpose of this Thesis is to evaluate the damage prevention potential of ballistic personal protections and achieve further knowledge on some of the impact-related head injury mechanisms. To fulfill these objectives, a numerical human head model is developed from medical images and subsequently calibrated using experimental data from the literature.

Explicit finite element analysis has been the main tool employed along this study and therefore a deep connaissance of its working principles and implementation is necessary to achieve realistic and repeatable results. For this reason a part of this Thesis will be devoted to this numerical methodology focusing on the most relevant aspects to non-linear impact dynamics. This theoretical basis will be extrapolated to specific impact problems, departing from simpler scenarios and giving rise to more sophisticated models.

\subsection{Organisation of the Thesis}

This Thesis is structured in ten chapters, arranged into five thematic blocks: state of the art in head biomechanics, numerical modeling of composite materials under impact conditions (including a description of the explicit finite element method), evaluation of Behind Helmet Blunt Trauma (BHBT), development of human head numerical models and evaluation of non-lethal projectiles (NLP). The current chapter presents an overview of the main objectives of this work and the justification of its research interest. Chapter 2 deepens into the existing knowledge on human head biomechanics based on published works in the literature. The first sections consist of a description of the main 
tissues that conform the human head paying special attention to their mechanical properties. Then, the concept of head injury is introduced from a clinical point of view and the focus is set on the relations between different mechanical parameters and specific lesions, which give birth to the so-called head injury criteria.

Chapter 3 is also descriptive and it points out the fundamental aspects of the explicit finite element method. Departing from the development of the equations for non-linear transient dynamics, the working principles of this method are presented. It is culminated with an explanation of the contact interaction approaches in FEM as this feature is tightly related to subsequent chapters that involve impact simulation.

Chapter 4 couples different approaches to address low-velocity impact on composite plates. Experimental methods (Charpy pendulum tests plus high speed recording and Digital Image Correlation, DIC) are employed to construct a numerical model capable of replicating the impact behaviour of a composite plate by means of explicit FE analysis. The introduction of composite materials at this stage of the Thesis is relevant for the development of posterior chapters dealing with personal protections.

Chapters 5 to 8 comprise the main body of this Thesis, which is head injury prediction based on numerical simulation. An image-based human head model is developed and validated against impact tests from the literature in Chapter 5 , and later coupled to a composite combat helmet to replicate the environment of ballistic impacts (Chapters 6 and 7 ). In Chapter 6, the protection simulated is an aramid combat helmet subjected to high-velocity impact from a $9 \mathrm{~mm}$ bullet. Current helmet acceptance protocols and sizing policies are discussed. Chapter 7 presents a comparative study between the performance of different stack configurations of the helmet shell, employing different materials and layups. Finally, a more detailed numerical head model, including some relevant intracranial substructures, is developed and presented in Chapter 8. It is employed in the FE reconstruction of a clinical case of accidental fall in order to achieve a tolerance criteria for a specific head injury: subdural hematoma.

Chapter 9 consists of a study of the behaviour of non-lethal projectiles performed in conjuntion with the University of Strasbourg (UNISTRA).

Finally, Chapter 10 collects the main contributions of this Thesis and proposes challenges that could be reached in the future if following the current research line. 



\section{Chapter 2}

\section{Head biomechanics}

It pays to keep an open mind, but not so open your brains fall out.

- Carl Sagan

Head is one of the most complex structures in the human body. Constituted by a wide set of living tissues along all typologies (muscular, epithelial, connective and nervous), it holds and protects the core of the central nervous system (CNS).

Understanding head biomechanics is a landmark that enhances the development of more effective surgical procedures, treatments and, specifically, better risk prevention systems. Nonetheless, further knowledge on the behaviour of head tissues has always been a challenge and the evidence of intra and interspecimen variability hinders the achievement of a generallistic characterisation.

In this chapter, some of the most relevant tissues in the human head are described from an anatomical and mechanical point of view based on preceeding studies from the literature. This descriptive part is followed by a review on several injury criteria based on mechanical parameters, which will be essential for the comprehension of posterior sections in this Thesis, in which head injury caused by impact will be addressed. 


\subsection{Mechanical behaviour of the skull}

The human skull is the structural basis of the head and protects the brain. It is an intricate system which comprises 22 different bones, some of which are movable. A schematic representation of an average human skull is depicted in Fig. 2.1 In this section the focus will be set mainly on the bones conforming the cranial vault, as they acquire great relevance in the protection against impact trauma.

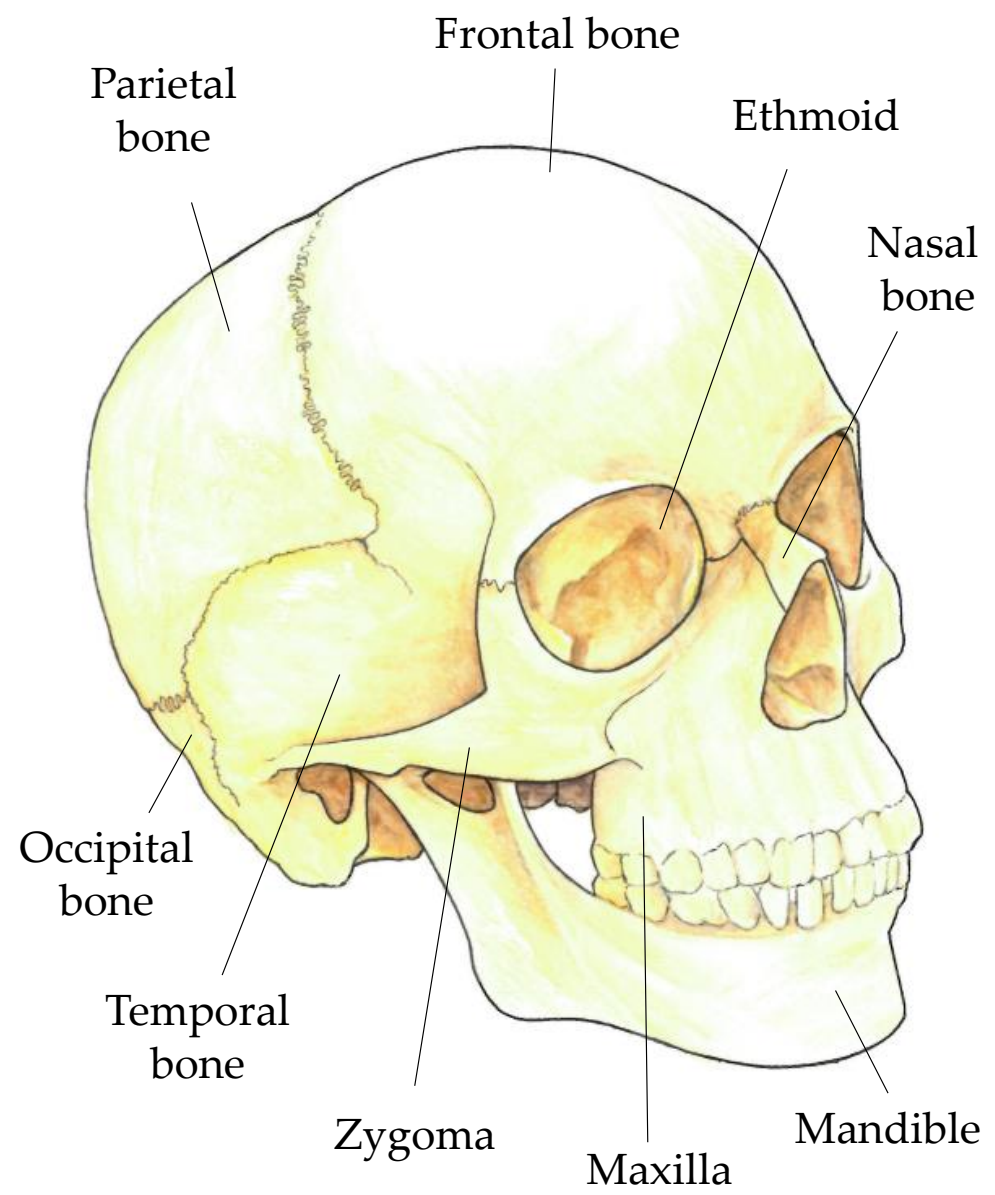

Figure 2.1: Human skull bones. 
Due to its complex geometry and heterogeneous composition, there are several studies addressing the morphological features of the human skull. Nonetheless, this section will set the focus on its mechanical properties based on experimental studies taken from the literature.

\subsubsection{Studies on bone samples}

Some studies from the early seventies attempted to characterise mechanically the cranial bone, either as a sandwich structure or each layer separately (see Fig. 2.2). To this purpose, bone specimens were normally obtained from postmortem human subjects (PMHS) calvaria and posteriorly machined and treated to acquire a specific shape in accordance to the test to be performed. Table 2.1 summarises some of the most relevant studies.

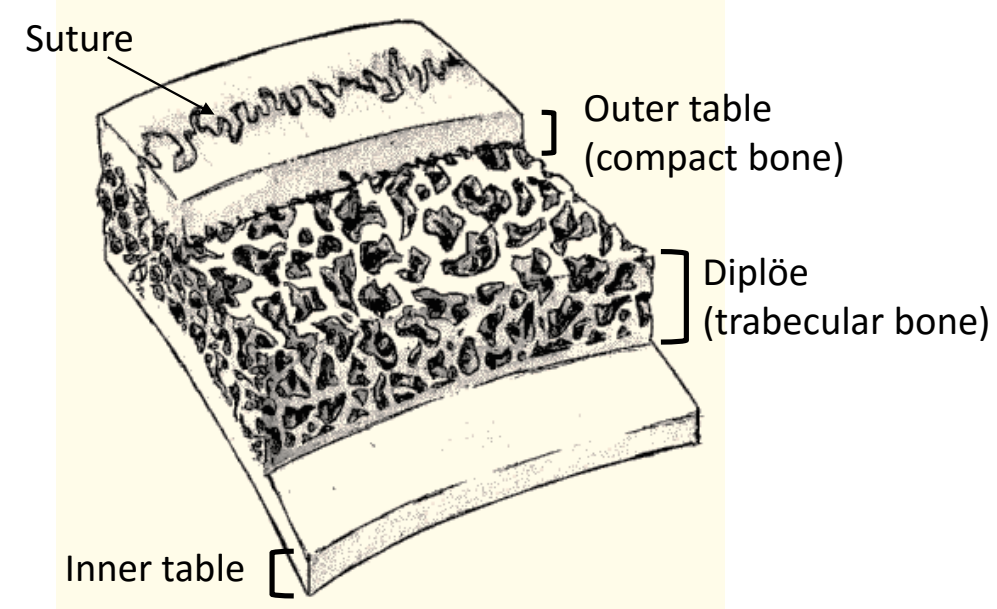

Figure 2.2: The three different bone layers of the cranium: compact bone tables and diplöe core.

Evans and Lissner (1957) [1] performed the first study measuring mechanical properties of the three-layered skull bone and one of the first which also comprised spongy bone. From tensile tests from parietal bone samples they obtained an average tensile failure stress $\left(\sigma_{u t}\right)$ of $70.53 \mathrm{MPa}$. This value was 
computed under the assumption that the diplöe layer does not contribute to the tensile strength. Also, compression tests were carried out in two manners: first applying the load tangentially to the bone layers, and then in the perpendicular direction. This first set of experiments led to an average value of $\sigma_{u c}$ of $-152.23 \mathrm{MPa}$ and the second set to a value of $-167 \mathrm{MPa}$. Finally, isolated diplöe specimens were tested under compression, resulting in an average $\sigma_{u c}$ of $-25.10 \mathrm{MPa}$, the first reported value of diplöe compressive strength in the literature.

A decade later, Robbins and Wood (1969) [2] deepened into the mechanical characterisation of skull bone by subjecting cranial compact bone samples to cyclic uniaxial loading that were finally loaded in monotonic tension until failure. Their results revealed the same elastic modulus for both tension and compression (average E of $14.55 \mathrm{GPa}$ ), also suggesting viscoelastic behaviour of the compact bone. As for tensile failure, it was determined that skull compact bone behaves as a brittle material with very little yielding before failure, reporting an average $\sigma_{u t}$ of $65.50 \mathrm{MPa}$, close to the value reported by [1] for parietal bone. To assess the structural behaviour of the skull bone as a multi-layered stucture, they harvested skull bone samples that included both the inner and outer tables and also the diplöe core and submitted them to uniaxial unconfined compression. $E$ and $\sigma_{u c}$ presented values of $1.39 \mathrm{GPa}$ and -36.54 MPa, respectively. However, the authors reported a great dispersion in the elasticity modulus of the structure, which was related to the amount of trabecular bone present in the sample.

A deeper study was performed by McElhaney et al. (1970) [3], who tested cranial samples under different loading conditions: tension, compression, shear and torsion, all of them under a quasistatic regime. In agreement with the findings of [2], they found a wide range of mechanical responses in the different specimens due to the regional variations in the trabecular structure, thus making this layer difficult to characterise, as opposed to the compact bone tables. From their study they concluded that skull bones are transversely isotropic with increased stiffness in the tangential directions to the skull surface, statement that agrees with the observations performed by Dempster (1967) [4] about the randomly-oriented microstructure of compact bone.

The first study that performed bending in layered cranial bone samples corresponds to Hubbard (1971) [5], who applied layered beam theory to compute the flexural stiffness and strength from three point bending (tpb) tests. 
This theory appeared to be suitable to relate both the material properties and the structural geometry of cranial bone. In the same study, four point bending tests were performed until the fracture of the sample, which presented a similar trend in all the cases: failure initiation at the inner compact bone table (submitted to tension) and crack propagation through dipöe and the outer table in a direction perpendicular to the surface.

Rate dependency on the mechanical properties of skull bone was first studied by Wood (1971) [6]. Taking compact bone specimens from the frontal, temporal and parietal regions, they performed tensile tests under different loading rates $\left(0.005-150 \mathrm{~s}^{-1}\right)$. Their results revealed low variations in the elasticity modulus along the whole number of samples at a given loading rate and an increase of its mean value when increasing the strain rate (from an average of 10.34 up to $20.06 \mathrm{GPa}$ ). Breaking stress and strain were also rate dependent $\left(\sigma_{u t} \in[48.26,127.55] \mathrm{MPa}\right)$, whereas the energy absorbed up to failure remained almost unaffected.

Later, Delille (2007) [7] performed tpb tests over 380 skull specimens (comprising the three bony layers) with the purpose of finding an adequate surrogate material for the human cranium able to give a more biofidelic approach than current dummies. To calculate mechanical properties they applied beam theory incorporating a correction parameter based on bone mineral fraction, in order to take into account the hollow structure of the trabecular lattice of the diplöe. Results were summarised according to each skull region, obtaining an elasticity modulus of 5.00 GPa for left parietal bone, $4.90 \mathrm{GPa}$ for right parietal bone and 3.79 GPa for frontal bone.

Motherway et al. (2009) [8] aimed at characterising the dynamic behaviour of skull bone by performing three point bending tests on cranial samples at three different velocities $\left(0.5,1\right.$ and $\left.2.5 \mathrm{~ms}^{-1}\right)$. The elastic modulus was calculated by appliying Timoshenko's beam theory with the assumption of a rectangular cross-area. Their results revealed a positive correlation between both $E$ and the force to failure and the loading rate. They also looked for relationships between the mechanical parameters and the microstructure of the spongy bone (which was characterised by means of $\mu \mathrm{CT}$ scan images) observing a wide range of variation in the porosity percent and the bone volume fraction between different craniums. No strong correlations were found based on these variables except for an increase in elastic modulus and bending stiffness for greater bone volume fraction values. 
Following the same line, Auperrin et al. (2014) [9] tested a wider sample of skull specimens (351) from different cranial regions of 21 subjects under three point bending. The load applied was cyclic and remained within the elastic domain. Analogously to $[7,8]$, theory of hollow beams was applied, calculating the porosity of the trabecular lattice from the sample ash density. Although they did not find direct relations between the elastic modulus and the age of the cranial donors (in the range between 52 to 95 years), they found a strong dependence of the apparent elasticity modulus on the location inside the cranium. The frontal bone presented the lowest values $\left(E_{\text {app }}\right.$ of $3.81 \pm 1.55$ $\mathrm{GPa})$ followed by the parietal $(5.00 \pm 3.12 \mathrm{GPa})$ and finally the temporal $(9.70$ $\pm 5.75 \mathrm{GPa})$. In view of these results, they proposed an exponential relation between $E_{\text {app }}$ and the product of bone thickness and its apparent density.

In a recent study, Boruah et al. (2017) [10] carried out tensile tests over cranial cortical bone samples at a rate of $100 \mathrm{~s}^{-1}$. The resultant stress-strain curves revealed a non-linear trend, which the authors attribute to microcracking, therefore compromising the accuracy of the measured value of the elastic modulus. Despite that fact, they concluded that both tensile stress and strain at failure differed substantially between frontal and parietal bone. One of their most novel finding was the difference in elastic modulus between the upper (average 17.5 GPa) and the lower calvarium (19.4 GPa). 


\begin{tabular}{|c|c|c|c|c|}
\hline Reference & $\begin{array}{l}\text { Sample } \\
\text { size }\end{array}$ & Test type & Location & Results \\
\hline $\begin{array}{l}\text { Evans and } \\
\text { Lissner } \\
(1957)[1]\end{array}$ & & $\begin{array}{l}\text { Uniaxial tension } \\
\text { and compression }\end{array}$ & Parietal & $\begin{array}{l}\sigma_{\mathrm{ut}} \approx 70.53 \mathrm{MPa} \\
\sigma_{\mathrm{uc}} \approx-152.23 \mathrm{MPa} \\
(\text { cranial bone) } \\
\sigma_{\mathrm{uc}} \approx-25.10 \mathrm{MPa} \\
\text { (diplöe) }\end{array}$ \\
\hline $\begin{array}{l}\text { Robbins } \\
\text { and Wood } \\
(1969)[2]\end{array}$ & & $\begin{array}{l}\text { Uniaxial tension } \\
\text { and compression }\end{array}$ & & $\begin{array}{l}E \approx 14.55 \mathrm{GPa} \\
\sigma_{\mathrm{ut}} \approx 65.50 \mathrm{MPa} \\
(\text { compact bone }) \\
E \approx 1.39 \mathrm{GPa} \\
\sigma_{\mathrm{uc}} \approx-36.54 \mathrm{MPa} \\
\text { (cranial bone) }\end{array}$ \\
\hline $\begin{array}{l}\text { Wood } \\
(1971)[6]\end{array}$ & 380 & Uniaxial tension & Various & $\begin{array}{l}E_{\text {mean }} \approx 10.34 \mathrm{GPa} \\
\sigma_{\mathrm{ut}} \approx 48.26 \mathrm{MPa} \\
\text { (quasistatic rate) } \\
E_{\text {mean }} \approx 20.06 \mathrm{GPa} \\
\sigma_{\mathrm{ut}} \approx 127.55 \mathrm{MPa} \\
\text { (quasistatic rate) }\end{array}$ \\
\hline $\begin{array}{l}\text { Hubbard } \\
(1971)[5]\end{array}$ & 61 & $\begin{array}{l}\text { Three-point } \\
\text { bending }\end{array}$ & Various & $\begin{array}{l}E_{\text {eq }} \approx 15.3 \pm 7.3 \mathrm{GPa} \\
(\text { experimental) } \\
E_{e q}=9.238 \mathrm{GPa} \\
\text { (beam theory) } \\
G_{d i p l} \approx 111.7 \pm 23.4 \mathrm{MPa} \\
\text { (experimental) } \\
G_{d i p l}=137.9 \mathrm{MPa} \\
\text { (beam theory) } \\
\varepsilon_{u t}=0.005\end{array}$ \\
\hline $\begin{array}{l}\text { Delille } \\
(2007)[7]\end{array}$ & 380 & $\begin{array}{l}\text { Three-point } \\
\text { bending }\end{array}$ & $\begin{array}{l}\text { Frontal } \\
\text { Left parietal } \\
\text { Right pari- } \\
\text { etal }\end{array}$ & $\begin{array}{l}E_{\text {app }} \approx 3.79 \mathrm{GPa} \\
E_{\text {app }} \approx 5.00 \mathrm{GPa} \\
E_{\text {app }} \approx 4.90 \mathrm{GPa}\end{array}$ \\
\hline $\begin{array}{l}\text { Motherway } \\
\text { et al. } \\
(2009)[8]\end{array}$ & 61 & $\begin{array}{l}\text { Three-point } \\
\text { bending }\end{array}$ & Various & $\begin{array}{l}E \approx 7.46 \pm 5.39 \mathrm{GPa} \\
\left(\text { dynamic rate } 0.5 \mathrm{~ms}^{-1}\right) \\
E \approx 10.77 \pm 9.38 \mathrm{GPa} \\
\left(\text { dynamic rate } 1.0 \mathrm{~ms}^{-1}\right) \\
\left.E \approx 15.54 \pm 10.29 \mathrm{GPa}^{-1}\right) \\
\left(\text { dynamic rate } 2.5 \mathrm{~ms}^{-1}\right)\end{array}$ \\
\hline \multirow{2}{*}{$\begin{array}{l}\text { Auperrin } \\
\text { et al. (2014) } \\
{[9]}\end{array}$} & 351 & $\begin{array}{l}\text { Three-point } \\
\text { bending }\end{array}$ & $\begin{array}{l}\text { Frontal } \\
\text { Parietal }\end{array}$ & $\begin{array}{l}E_{\text {app }} \approx 3.81 \pm 1.55 \mathrm{GPa} \\
E_{\text {app }} \approx 5.00 \pm 3.12 \mathrm{GPa}\end{array}$ \\
\hline & & & Temporal & $E_{\mathrm{app}} \approx 9.70 \pm 5.75 \mathrm{GPa}$ \\
\hline
\end{tabular}




\subsubsection{Studies on whole cranium}

While experimental testing of bone samples provides useful information of the regional properties of the skull and its behaviour under different loading conditions, impact testing employing whole craniums can give more insight about its mechanical behaviour as a structure, especially regarding macroscopical failure.

Some of the first studies addressing skull fracture were carried out at the end of the 1940s [11-13]. In these works, fracture was characterised by means of the so-called stresscoat technique. In 1950, Gurdjian et al. [14] summarised the most relevant conclusions drawn from these previous studies to give a detailed overview of the process of skull fracture initiation and to establish different fracture typologies according to the impact energy delivered in the blow. To this purpose, they conducted experimental tests on 46 unembalmed postmortem heads which were dropped onto a flat surface from different heights.

A posterior work by Nahum et al. (1968) [15], aimed at determining skull bone tolerances by means of experimental tests on different locations on the head of ten embalmed and unembalmed specimens. The impact blows were delivered with different energies, thus engaging the apparition of various levels of skull fracture severity. They established tolerance values in terms of peak force for the different regions, distinguishing between a threshold value for significant fractures (frontal $4893 \mathrm{~N}$, temporoparietal $2446 \mathrm{~N}$ and zygoma $1000 \mathrm{~N}$ ) and the mean force value leading to fracture (frontal $4003 \mathrm{~N}$, temporoparietal $2001 \mathrm{~N}$ and zygoma $889 \mathrm{~N}$ ). Lower tolerances were found for the female specimens. Additionally, the authors concluded that embalming the specimens did not significantly affect skull tolerance in the areas were soft tissues (mainly cartilage) do not have a relevant contribution. Nonetheless, keeping the soft tissue surrounding the cranium tends to mitigate the impact severity of skull bone, requiring greater force values for fracture initiation.

Hodgson et al., (1970) [16] recreated experimentally the scenario of a frontal car crash. They conducted impact tests on 12 PMHS bodies using two cylindrically shaped impactors with diferent radius, with the subject head being struck at its frontal site. The loading procedure consisted of applying several impacts on the same specimen increasing each time the initial energy until achieving skull fracture. After the first blow on each subject, the scalp was replaced by a surrogate material. The greater radius impactor resulted in 
remote linear fractures, which involved the inbending of the inner table and surrounding outbending areas where the cracks initiate, coinciding with the findings of Gurdjian et al. [11]. In view of these results, this impactor behaved as a blunt surface, and the resulting loads for fracture were set between $4225 \mathrm{~N}$ and $7339 \mathrm{~N}$. For the cases struck by the smaller radius impactor, both linear or localised elliptical fractures were obtained, therefore classifying this impactor type in the transition between blunt surface and a sharp device. Peak forces for this case ranged between $5560 \mathrm{~N}$ and $7117 \mathrm{~N}$.

Gathering the experimental data from the literature up to that moment, Melvin and Evans (1971) [17] assessed skull fracture from the point of view of strain energy. They characterised the three most common types of skull fractures obtained from automotive crashes. The belonging to one fracture typology or another mainly depends on the impactor's contact surface and shape, as well as the force delivered. With small impactor areas, the most likely outcome is a penetration fracture, in which failure occurs at a localised region coinciding with the impact site, and its propagation is dominated by the diplöe compressive strength. Impactor surfaces of around 1 in $^{2}$ often lead to comminuted-depressed fractures; in these, a bending moment at the loading region causes a tensile failure in the inner compact table, resulting in a local linear fracture that engages the subsequent depression of the impacted area. Finally, if the impactor presents a greater area, the load tends to distribute more uniformly along the cranial vault (and so does the strain energy) until concentrating at the skull weaker regions up to the threshold level of crack initiation. This last case is the remote linear fracture. Impacts with intermediate characteristics may result in a combination of the presented fracture patterns.

A specific skull trauma affecting the base of the cranium, i.e. basilar skull fracture (BSF) was studied by McElhaney et al. in 1995 [18]. To this purpose, they performed three series of experimental tests using PMHS. The first consisted on vertical drops of five unembalmed heads, in such a manner that the impact took place in the chin of the specimen. No basilar fractures were found with this method, fractures in the mandible were obtained instead at a force value of $5390 \pm 990 \mathrm{~N}$. They also measured the energy to reach mandibular fracture and discovered a great variation depending on the stiffness of the impacting surface, reporting a value of $11.4 \mathrm{~J}$ to achieve fracture with a rigid surface against $119 \mathrm{~J}$ when it involves a compliant one. Their second set of tests employed the same specimens than the first one, but inserting a steel 
tube in the base of the skull to provide tensile loading (simulating the effect of the neck constraint) and applyind the load on the mandibular region. In this case four basilar skull fractures were obtained being $4300 \pm 350 \mathrm{~N}$ and $4160 \pm 700 \mathrm{~N}$, the mean forces at fracture measured in the neck and in the mandible, respectively. The authors hypothesize that the fracture tolerance for the base of the skull should lie between both values. The measured energy absorbed up to failure was $13.0 \pm 1.7 \mathrm{~J}$. Finally, their third set of experiments involved drop tests of 11 human head-neck specimens mounted on a certain mass to simulate the inertia of the torso. The impact, therefore, took place at the cranial vault. This loading mechanism did not result in BSF but in injuries to the upper neck, reporting much lower tolerance values for this part than for the base of the skull.

Allsop et al. (1991) [19] conducted impact tests on 31 PMHS heads using the testing set-up developed in a previous work [20], consisting on a striking device that hits the specimen with sufficient energy to cause skull fracture. This device was mounted with two different striking surfaces: a flat plate of 5 by $10 \mathrm{~cm}$, and a circular plate of $2.54 \mathrm{~cm}$ diameter. The latter delivered the impact in two different head sites. An average fracture force value of 12.39 $\mathrm{kN}$ was obtained for the rectangular anvil, while for the circular anvil this value fell to 4.99 and $5.4 \mathrm{kN}$, for the two impact sites. This reduction in the critical force value is mainly due to the difference in the impactor surface value, which directly involves a difference in contact area. Fracture force values from the load cell were validated with records of acoustic emissions. Finally, they also computed cranial stiffness values from the dynamic loading, observing a dependence on contact area, analogously to fracture force.

In 1995, Yoganandan et al. [21] published an experimental study on 12 unembalmed PMHS heads, which were tested both at quasistatic and at dynamic rates. The testing device allowed to constrain the movement of the specimen and adjust the location of the impact, which is delivered by an electrohydraulic piston with a hemispherical anvil. Force and displacement up to failure were recorded, and then skull stiffness and the energy absorption until fracture were computed, resulting in a mean stiffness value of $812 \mathrm{Nmm}^{-1}$ for quasistatic rates and $4023 \mathrm{Nmm}^{-1}$ for dynamic rates. The mean absorbed energy for each group is $33.5 \mathrm{~J}$ and $28 \mathrm{~J}$, respectively. Differences between the fracture patterns and the force deflection curves were observed between the two test groups. Quasistatically loaded specimens revealed force-deflection curves with several peaks, which were associated to microfractures and yield- 
ing phenomena, while the dynamically loaded specimens presented only one peak force point, suggesting sudden collapse.

Delye et al. (2007) [22] proposed the energy absorbed up to failure as a robust predictor for skull fracture. Using a test set-up previously validated [23] and consisting of a double pendulum which allowed specimen displacement in the impact direction, 18 unembalmed PHMS heads were tested at three different velocity ranges in the front. Failure was considered to happen at the peak point of the force-displacement curve. Mean values of the absorbed energy for each loading rate were: $22.53 \mathrm{~J}$ for high velocity $\left(6.95 \mathrm{~ms}^{-1}\right), 24.78$ for intermediate velocity $\left(5.21 \mathrm{~ms}^{-1}\right)$ and 8.98 for low velocity $\left(3.60 \mathrm{~ms}^{-1}\right)$. The authors proposed then a tolerance energy value of frontal bone of $22-24 J$ independently of the impact velocity. Additionally, the force-displacement curves from the tests appeared to be velocity-dependent, finding (for the low and intermediate velocities test groups) a response curve similar to the one obtained by Yoganandan et al. [21] for their quasistatic experiments. In these cases, a clear change of slope is manifested before fracture, while for the higher velocity tests, the stiffness remains constant up to failure, which seems to indicate that fracture occurs instantaneously.

The fact of relating experimental peak force with fracture force was criticised by Cormier et al. (2011) [24]. They set the fracture initiation value based on the acoustic emission history recorded from each of their impact experimental tests on 27 PMHS. This approach lead to considerably more conservative fracture risk curves, setting a $50 \%$ risk in the range of 1885 and 2405 N. In view to these results, the authors suggest that previous works dealing with frontal bone tolerance tend to overestimate the fracture initiation force value.

The most relevant features of the previously described studies are summarised in Table 2.2. It is noticeable the great dispersion in fracture tolerances between studies, which highlights the difficulty of achieving an accurate characterisation of this phenomenon. 


\begin{tabular}{|c|c|c|c|c|}
\hline Reference & $\begin{array}{l}\text { Sample } \\
\text { size }\end{array}$ & Test type & Location & Fracture tolerance \\
\hline $\begin{array}{l}\text { Nahum et al. } \\
(1968)[15]\end{array}$ & 10 & Impact & $\begin{array}{l}\text { Frontal } \\
\text { Temporoparietal } \\
\text { Zygoma }\end{array}$ & $\begin{array}{l}F_{\text {frac }} \approx 4893 \mathrm{~N} \\
F_{\text {frac }} \approx 2446 \mathrm{~N} \\
F_{\text {frac }} \approx 1000 \mathrm{~N}\end{array}$ \\
\hline $\begin{array}{l}\text { Hodgson et al. } \\
(1970)[16]\end{array}$ & 12 & $\begin{array}{l}\text { Impact against } \\
\text { cylindrical anvil }\end{array}$ & Frontal & $\begin{array}{l}F_{\text {frac }} \in[4225-7339] \mathrm{N} \\
\text { (larger radius) } \\
F_{\text {frac }} \in[5560-7117] \mathrm{N} \\
\text { (smaller radius) }\end{array}$ \\
\hline $\begin{array}{l}\text { Allsop et al. } \\
(1991)[19]\end{array}$ & 31 & Drop tower & Temporoparietal & $\begin{array}{l}F_{\text {frac }} \approx 12390 \mathrm{~N} \\
(\text { rectangular anvil }) \\
F_{\text {frac }} \in[4.99-5.40] \mathrm{N} \\
(\text { circular anvil })\end{array}$ \\
\hline $\begin{array}{l}\text { Yoganandan } \\
\text { et al. (1995) [21] }\end{array}$ & 12 & $\begin{array}{l}\text { Electrohydraulic } \\
\text { piston }\end{array}$ & Various & $\begin{array}{l}F_{\text {frac }} \approx 6399 \pm 1134 \mathrm{~N} \\
\text { (quasistatic rate) } \\
F_{\text {frac }} \approx 11938 \pm 885 \mathrm{~N} \\
\text { (dynamic rate) }\end{array}$ \\
\hline $\begin{array}{l}\text { McElhaney } \\
\text { et al. (1995)[18] }\end{array}$ & 12 & Drop tests & $\begin{array}{l}\text { Mandible } \\
\text { Skull base }\end{array}$ & $\begin{array}{l}F_{\text {frac }} \approx 5390 \pm 990 \mathrm{~N} \\
E_{\text {abs }} \approx 11.4-119 \mathrm{~J} \\
F_{\text {frac }} \text { between } 4300 \pm \\
350 \mathrm{~N} \\
\text { and } 4160 \pm 700 \mathrm{~N}\end{array}$ \\
\hline $\begin{array}{l}\text { Delye et al. } \\
(2007)[22]\end{array}$ & 18 & $\begin{array}{l}\text { Double } \\
\text { pendulum[23] }\end{array}$ & Frontal & $\begin{array}{l}E_{\text {abs }} \approx 22-24 \mathrm{~J} \\
\text { (for all velocities) }\end{array}$ \\
\hline $\begin{array}{l}\text { Cormier et al. } \\
(2011)[24]\end{array}$ & 27 & $\begin{array}{l}\text { Free fall } \\
\text { impactor }\end{array}$ & Frontal & $\begin{array}{l}F_{\text {frac }} \approx 1885-2405 \mathrm{~N} \\
(50 \% \text { fracture risk })\end{array}$ \\
\hline
\end{tabular}

Table 2.2: Summary of published results on skull fracture load.

\subsection{Brain tissue biomechanics}

Human brain is the kernel of the central nervous system as it collects the sensorial signals and commands the required responses to the rest of the body. 
Located in the interior of the cranial vault, it is primary conformed of neurons, interconnected by axons and dendrites. Macroscopically, it accounts for three large structures: the cerebrum, containing white and gray matter and divided in two hemispheres, the cerebellum, that controls body motion, and the brainstem, which commands visceral automatic functions. These are at the same time subdivided in many other structures, some of which are illustrated in Fig. 2.3.

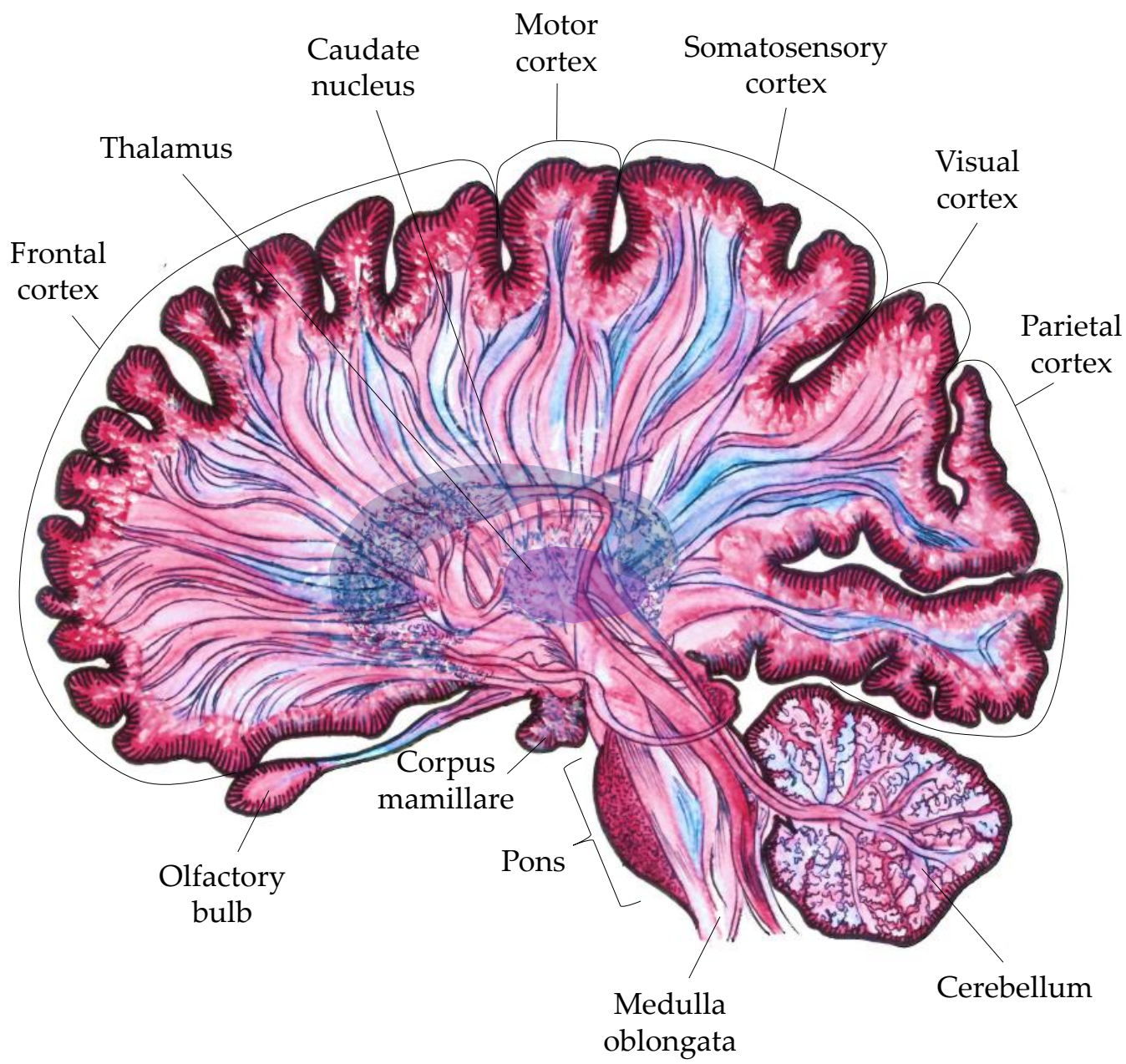

Figure 2.3: Neural tissue and structures of the brain. 
As performed with the skull bones, in this section the human brain will be assessed from the biomechanical standpoint, and therefore it will focus on its mechanical behaviour, which is a key factor for understanding the development of the most frequent head injuries. First, a summary of the mechanical properties of the brain tissue gathered from the literature by means of both experimental testing and imaging techniques will be presented. Secondly, and based on these properties, the main attempts to define the constitutive modeling of the brain tissue will be summarised.

\subsubsection{Experimental approaches}

\section{Testing on brain samples}

Mechanical characterisation of human brain tissue has always been a complex problem due to its inherent non-homogeneities. The irregularity of the brain geometry as well as the presence of differenciated structures (both in the macro and micro scales) embedded inside its lattice represented a challenge in order to establish valid constitutive properties for this living tissue. The first studies addressing the biomechanics of the brain considered its behaviour as similar to a gel-like material with a high water content surrounded by a stiffer cover (pia matter) and reinforced with multiple blood vessels [25]. Nonetheless, experimental studies until the first decade of the 21st century presented dispaired values for the mechanical constants of the brain tissue, sometimes differing several orders of magnitude. These discrepancies can be caused by some factors related to the experimental testing procedure [26-29]. First, studies performing in vitro tests are subjected to the limitations of the time of death of the subject, the harvesting and conservation procedure of the samples, the absence of blood flow, among others. Of course, due to the difficulty of obtaining human brain specimens, many of the existing studies are based on animal tests, whose extrapolation to human is not straightforward for the majority of the species.

In 1969, Fallenstein et al. [25] performed the first attempt to determine shear properties of the human brain under dynamic loading in in vitro conditions. To that purpose, they conducted low-frequency oscillatory shear tests on 13 rectangular samples harvested from the white matter of eight postmortem human subjects. From these experiments they obtained a value of the brain's dynamic elastic shear modulus $\left(G^{\prime}\right)$ of about $0.6-1.1 \mathrm{kPa}$ and a loss modulus 
$\left(G^{\prime \prime}\right)$ of 0.35-0.6 kPa. The following year, Galford and McElhaney (1970) [30] performed unconfined compression experiments on both human and rhesus monkey brain samples. Relaxation tests revealed an initial elastic modulus of $10.34 \mathrm{kPa}$ for monkey brain and $6.55 \mathrm{kPa}$ for human brain. This same kind of experimental testing was employed by Estes and McElhaney (1970) [31] to characterise dynamic brain behaviour under compression. Results from this last study were later employed in other works for the development of a constitutive law for the homogenised brain tissue.

Posteriorly, Shuck and Advani (1972) [32] conducted oscillatory shear tests of human brain samples. Histological examination of the samples encouraged the authors to assume that microstructural components of the brain tissue had not a size sufficient enough to affect the global response of the tissue and therefore that brain behaves as an isotropic material. Additionally and counterintuitively, experiments revealed that gray matter had more directional sensitivity (thus being more anisotropic) than white matter, whose measured shear moduli were very similar for the three directions tested. The averaged shear modulus of white matter along the three directions was slightly higher than the reported for gray matter.

Miller and Chinzei (1997) [33] conducted in vitro unconfined compression tests on swine cylindrical brain samples. Testing velocities were varied from 0.005 to $500 \mathrm{~mm} \mathrm{~min}^{-1}$, range upon which the stress-strain response was significantly variable. The authors aimed at obtaining the brain response at surgical (low) rates and therefore their values should not be directly extrapolated to injury-like environments. Additionally, the arachnoid membrane was kept in the cylindrical samples, a fact that affects the overall tissue response. Employing the experimental in vitro data from this work, further studies by Miller (1999) [34] and Miller et al. (2000) [35] tried to develop and validate a constitutive law for the in vivo brain tissue suitable for surgical simulation. An indentation test on a swine alive specimen allowed the validation of their methodology [35].

In an attempt to compare the response of brain tissue with commonly employed surrogate materials (gelatine mixtures and silicone gel), Brands et al. (1999) [36] performed small-strain oscillatory shear tests. Porcine samples were chosen for brain tissue characterisation, and they were cut in such a manner that no anisotropic effects arose. The strain limit for elasticity in brain tissue was established at 0.01 , while for the surrogate materials it was 
significantly higher ( 0.1 for silicone gel and nearly perfect elastic behaviour for gelatins). Dynamic shear modulus of brain tissue grew from 500 to $665 \mathrm{~Pa}$ in the frequency range of 0.1 to $10 \mathrm{~Hz}$ and increased up to $1250 \mathrm{~Pa}$ at $260 \mathrm{~Hz}$. Their extrapolation to a wider frequency range based on the time/temperature superposition principle [37] revealed good agreement with the loss and storage modulus reported on both human and porcine brain at different rates (Fallenstein et al. [25], McElhaney et al. [38], Arbogast and Margulies [39], Peters et al. [37], and Thibault and Margulies [40, 41]). Nonetheless, Bilston et al. (2001) [42] performed an analogous testing sequence on bovine white matter samples and obtained a linear elastic strain limit one order of magnitude lower than [36]. The average storage and loss moduli were almost one order of magnitude greater $\left(G^{\prime} \approx 2500 \mathrm{~Pa}\right.$ and $G^{\prime \prime} \approx 850 \mathrm{~Pa}$ in [42] as opposed to $G^{\prime} \approx 500$ $\mathrm{Pa}, G^{\prime \prime} \approx 75 \mathrm{~Pa}$ in $\left.[36]\right)$.

Based on the testing procedure of oscillatory shear tests from [25], Arbogast and Margulies (1998) [26] aimed to mechanically characterise the brainstem, due to its important role in severe diffuse axonal injury. This structure was supposed to be more vulnerable to rotational loads either because of different material stiffness or due to the alignment of axonal fibers. Seven porcine cylindrical brainstem samples were tested at different strain levels $(2.5,5$ and $7.5 \%)$ in different shear directions. From their results, transverse isotropy of the brainstem was demonstrated, as the storage and loss moduli in the plane where fibers are parallel to the shear plane but perpendicular to the shear direction was significantly higher (10-20\%) than in the other two loading modes. Average moduli values reveal that the brainstem is about $80-100 \%$ stiffer than the cerebral hemispheres, finding that contradicts the suppositions of other works [43]. One relevant conclusion of the work was the lack of anisotropic experimental statistical evidence in the lowest test frequencies, fact that highlights the rate dependence of neural tissue and also suggests that at low loading rates the brainstem may behave as an isotropic material [32].

Prange and Margulies (2002) [44] aimed at giving light to the existing studies on Traumatic Brain injury (TBI) by obtaining the mechanical response of different porcine brain regions under unconfined compression inducing large strains, as other works postulate that neural damage occurs for deformations in the range of 0.2 . Their initial assumption for the study was the consideration of the gray matter as an isotropic material, as opposite to white matter, where axonal orientations lead to a transversely isotropic behaviour. Furthermore, the white matter samples tested in their work comprised different 
regions: the corona radiata and corpus callosum. This last is hypothesised to be constituted of highly uniaxially aligned axons. Compression tests were performed along the two principal directions of the samples and the subsequent results confirmed their hypothesis. In fact, almost no variation was found between the instantaneous shear moduli of the longitudinal and transversal directions in the gray matter samples (extracted from the thalamus) while in the corona radiata the difference was about the $40 \%$ and in the corpus callosum more than $90 \%$, thus revealing the anisotropic behaviour of these white matter structures. Additionaly, the authors concluded that the most suitable constitutive law for the studied brain regions was a modified Ogden model in conjunction with a Prony series for the time-dependence of the shear modulus.
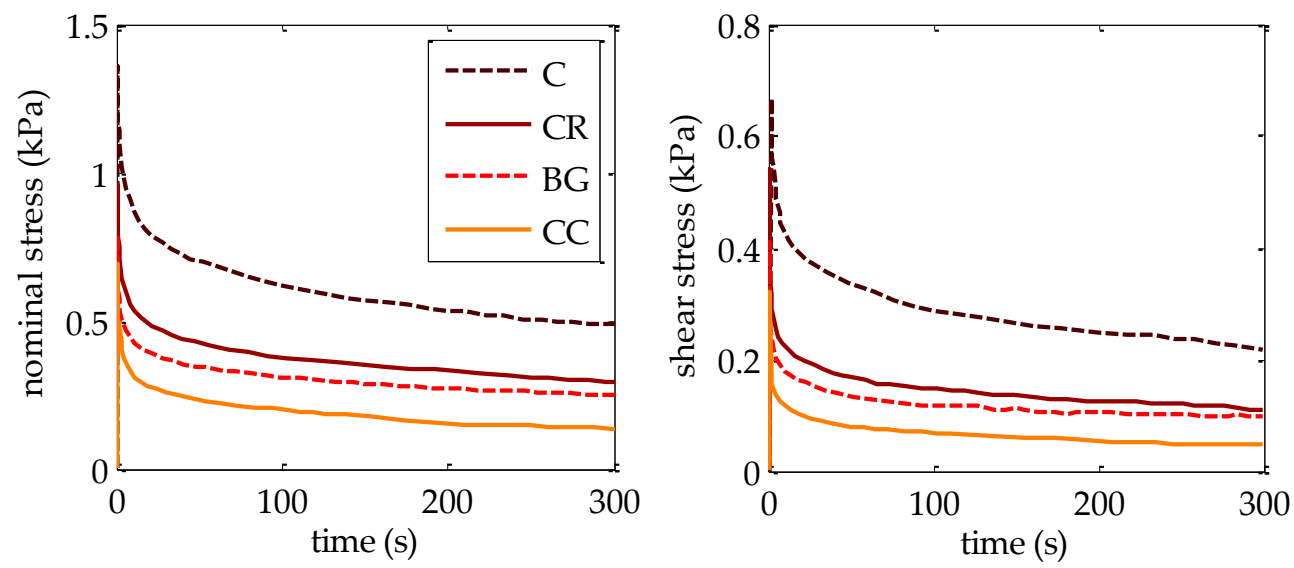

Figure 2.4: Typical stress relaxation curves for brain tissue in compression (left) and shear (right). Experimental curves extracted from the tests conducted by Budday et al. [45] for different brain regions. $\mathrm{C}=$ cortex, $\mathrm{CR}=$ corona radiata, $\mathrm{BG}=$ basal ganglia, $\mathrm{CC}=$ corpus callosum.

Another set of oscillatory shear tests was carried out by Nicolle et al. (2014) [27] on both porcine and human brain samples from gray and white matter. Their study aimed to widen the range of test frequencies in order to gain insight in the brain response under automotive and non-penetrating ballistic events (frequencies about $1000 \mathrm{~Hz}$ ). Their testing protocol was divided into low frequency and high frequency experiments employing different devices. 
Comparing results from porcine and human samples the authors found no significant difference in terms of viscoelastic properties. Linear behaviour in all samples was observed for strain levels up to $1 \%$, and no evidence of the anisotropic behaviour of corona radiata was found. Frequency-dependence was corroborated by the results: white matter presented values of $G^{\prime} \approx 2.1 \mathrm{kPa}$ and $G^{\prime \prime} \approx 0.4 \mathrm{kPa}$ at $0.1 \mathrm{~Hz}$ and $G^{\prime} \approx 16.8 \mathrm{kPa}$ and $G^{\prime \prime} \approx 18.7 \mathrm{kPa}$ at $6310 \mathrm{~Hz}$. Gray matter appeared to be slightly stiffer than white matter for the regions tested.

In order to capture the large-strain behaviour of brain tissue, Hrapko et al. (2006) [28] performed in vitro shear and unconfined compression experiments on porcine brain samples. In agreement with previous studies, increasing loading rate was found to stiffen brain response. A strain value of 0.01 defined the elastic limit of the material, as found by Brands et al. [36] and Nicolle et al. [27]. Additionally, it was determined that brain samples did not completely recover their initial shape when strain levels higher than $20 \%$ were applied, even though no mechanical alteration indicating damage was visible in the strain range tested (until 50\%). Similarly, Prevost et al. (2011) [46] also examined the large-strain behaviour on brain tissue by in vitro unconfined compression tests on brain samples (comprising white and gray matter and sulci) from porcine specimens at strain rates from 0.01 to $1 \mathrm{~s}^{-1}$. The novelty in this latter work is the assessment of the brain's volumetric behaviour at different strain rates by means of image analysis, finding that increasing the strain rate led to greater values of the bulk modulus because interstitial fluid has less time to diffuse from the sample. They did not support the assumption of brain tissue as an incompressible material, as their results reported a change of volume in the samples up to the $12 \%$ at the maximum strain level $(50 \%)$.

Several experimental works have been published recently. For instance, Jin et al. (2013) [47] performed compression, tension and shear tests at different rates on 240 human brain samples comprising the gray matter (cortex and thalamus) and white matter (corpus callosum and corona radiata). Stiffening of the mechanical response was observed when increasing the strain rate, and dependency on the loading direction was found to be relevant in the white matter tissues. However, their results did not support the hypothesis of transversal isotropy for white matter from[26]. Regional dependency was also demonstrated, with white matter stiffness values overall being higher than gray matter and specifically in the case of the corona radiata. No specific numerical values were reported and the study was rather more qualitative than quantitative. Later, Forte et al. (2017) [48] studied gray and white matter 
human samples under compression-relaxation tests at different displacement rates at a 30\% strain level. Rate dependency of both tissues was confirmed with data showing up to five times higher stresses for the largest testing velocity. Overall, white matter was obtained to be 1.8 times stiffer than gray matter. Finan et al. (2017) [49] performed ex vivo microindentation tests, as it is claimed that this procedure is suitable for characterising mechanical properties of specific structures as long as the domain is relatively larger than the indenter tip. The strain rates tested ranged between 0.005 and $5 \mathrm{~s}^{-1}$. Based on the assumption of small strains, the viscoelastic properties of gray matter cortex, white matter cortex and the different regions of the hippocampus were obtained from 11 human donors. They found no significant differences in the behaviour of the different hippocampal structures and therefore this tissue was approximated as a continuum. Cortical gray matter presented lower stiffness values (about 60\%) than white matter cortex and the hippocamus, these two having a similar response. The conclusions achieved by this work, however, contradicted a posterior experimental/numerical study by Budday et al. (2017) [45]. In the later, human brain cubical specimens extracted from different regions (cortical gray matter (cortex), deep gray matter (basal ganglia), corona radiata and corpus callosum) were submitted to a sequence of loading modes: shear in two directions followed by unconfined compression and finally tension. From DTI imaging the authors assumed that the most likely candidate to show directional dependence was the corpus callosum due to the uniform axonal orientation in that structure. Nonetheless, results did not confirm the anisotropic behaviour of the corpus callosum and unexpectedly revealed more directional sensitivity in the cortex. However, all the tissues tested were assumed to be isotropic in light of the obtained results. As opposed to the conclusions of previous works [26, 48, 49], gray matter cortex showed the stiffest response, followed by corona radiata and basal ganglia and finally the corpus callosum as the most compliant. These discrepancies were attributed to specimen dimensions.

A more detailed work for the characterisation of the anisotropy of white matter was performed by Labus and Puttlitz (2016) [50]. As opposed to most of the previous existing experimental works on brain tissue dealing with uniaxial loading states, biaxial loading of ovine white matter samples was performed in this study. Additionally, the authors intended to relate structural properties of the axonal network to the mechanical response of the regions studied (corona radiata and corpus callosum). To that purpose, histological and trans- 
mission electron microscopy (TEM) analyses were performed to obtain three parameters: mean axonal orientation $\left(\theta_{\mathrm{m}}\right)$, axonal distribution $(\kappa)$ and axonal volume fraction $\left(f_{a}\right)$. Their results revealed an infinitesimal shear modulus of $480 \mathrm{kPa}$ and $460 \mathrm{kPa}$ for the corona radiata and corpus callosum, respectively, and histological parameters revealed an almost complete alignment of the axons in the corpus callosum and a considerable anisotropy in the corona radiata. The axonal volume fraction, however, did not have a major effect on the mechanical response.

\section{Image-based brain tissue characterisation}

The possibility of overcoming the main problems of ex vivo testing was introduced at the beginning of this century with the emergence of improved image-based techniques [51]. Specifically, magnetic resonance elastography (MRE) allowed the assessment of brain tissue mechanical properties with a non-invasive in vivo procedure, which was not directly possible with other imaging techniques such as radiography, CT or ultrasound [52]. In particular, the MRE technique is regarded as an innovative non-invasive tool to diagnose several diseases which are often manifested through the alteration of mechanical properties in given regions of the intracranial cavity [53-55]. Also, the diffusion tensor imaging technique permitted the morphological characterisation of the axonal bodies and the neuronal distribution along the brain structure. Axonal density and the orientation of each body play a determinant role in the underlying mechanical properties of white matter. Therefore, the response of the living intracranial tissues can be achieved directly on human subjects.

Kruse et al. (2008) [52] conducted a MRE study on 25 human healthy patients and estimated the brain shear modulus at a frequency of $100 \mathrm{~Hz}$, obtaining an average value of $13.6 \mathrm{kPa}$ for white matter and $5.22 \mathrm{kPa}$ for gray matter. Nonetheless, this procedure was only able to capture the mechanical properties of the periphery of the brain as the exciting shear waves attenuated towards its centre. The same year, an analogous work by Green et al. [56] on five volunteers reported opposite conclusions, with gray matter values of $G^{\prime}(3.1 \mathrm{kPa})$ higher than for white matter $(2.5 \mathrm{kPa})$ whereas $G^{\prime \prime}$ presented an average value of $2.5 \mathrm{kPa}$ in both regions. Discrepancies between both studies may arise in the process of obtaining the displacement fields, as in 
[56] a curl operator is applied in order to obtain a Helmholtz equation that would be posteriorly inverted, having removed the contribution of dilatational waves along the excited brain. Without the use of this operator, the opposed shear stiffness distribution is obtained: increasing from the brain's periphery towards the centre. The need of a robust valid inversion method for MRE displacement fields acquisition was therefore put into manifest. Specifically, most of the pioneer works in MRE on the brain tissue employ an inversion technique that is based on an isotropic mechanical model, which makes this methodology unable to detect directional dependencies of the brain tissue [57].

Johnson et al. (2013) [58] performed the first study characterising the mechanical properties of different regions of the white matter employing MRE. Magnetic resonance scans of seven healthy human volunteers submitted to shear pulses in the brain were collected in conjunction with the corresponding DTI dataset. Displacements due to the shear excitation were acquired and the regional mechanical properties of the tissue were obtained through finite element nonlinear inversion (NLI). Both the corpus callosum and the corona radiata of the subjects were extracted from the images through an atlas-based segmentation. Results revealed a greater stiffness in the corpus callosum than in the corona radiata, with storage shear moduli of about 3.09 and $2.78 \mathrm{kPa}$, respectively. Nonetheless, the loss modulus was significantly higher in the corona radiata $(1.97 \mathrm{kPa})$ than in the corpus callosum $(1.23 \mathrm{kPa})$. Inside each of these structures there were also regional differences in the viscoelastic properties, related to the axon number, diameter and orientation at each location. They summarised that higher $G^{\prime}$ values are related to greater axonal dimensions while $G^{\prime \prime}$ tends to increase where the axonal lattice is less uniaxially aligned.

Following the line of [58], a posterior work by Anderson et al. (2016) [57] also assessed the anisotropic behaviour of certain white matter regions (corpus callosum, corona radiata and the superior longitudinal fasciculus). In order to deal with directional dependent responses in the isotropic-based inversion technique for MRE, a multiaxial excitation was performed on the subject of study consisting of $90 \mathrm{~Hz}$ shear perturbations in the left-right (LR) and anteroposterior directions of the head. Different shear moduli were found in both the corpus callosum and corona radiata for the two directions tested, being both stiffer in the LR direction. This difference was found to be more pronounced in the corpus callosum, in agreement with experimental studies 
$[44,50]$. Overall, the corona radiata presented higher storage and loss moduli than the other regions tested $\left(G^{\prime} \approx 3.2 \mathrm{kPa}\right.$ and $\left.G^{\prime \prime} \approx 1.6 \mathrm{kPa}\right)$.

MRE-based characterisation of white matter anisotropy was also attempted in a recent study by Schmidt et al. (2018) [59] on ex vivo porcine samples excitated at different frequencies. They applied a local direct inversion (LDI) to estimate the shear modulus from the MR images even though the anisotropic properties were obtained from a finite element simulation. To be able to obtain the anisotropy level of the tissue, they induced pure transverse waves with radial propagation from the centre of the sample, being the wavefront perpendicular to the fiber direction. Therefore, as no tensile fiber excitation is performed, the propagation speed of the wave $(c)$ can be defined by the minimum shear modulus ( $\mu_{2}$, perpendicular to fibers) and the shear anisotropy factor $(\phi)$ through the expression:

$$
c^{2}=\frac{\mu_{2}}{\rho}\left(1+\phi \cos ^{2} \theta\right) \quad \phi=\frac{\mu_{2}}{\mu_{1}}-1
$$

Complementary dynamic shear tests were performed on 10 porcine samples at an excitation frequency between $20-30 \mathrm{~Hz}$ obtaining a minimum shear modulus $\mu_{2}$ of about $0.5 \mathrm{kPa}$ with $\phi \approx 0.125$. MRE tests at higher frequencies led to higher stiffness and anisotropy values: $\mu_{2} \approx 1 \mathrm{kPa}$ and $\phi \approx 0.27$ at 100 $\mathrm{Hz}, \mu_{2} \approx 1.9 \mathrm{kPa}$ and $\phi \approx 0.29$ at $200 \mathrm{~Hz}$, and $\mu_{2} \approx 2.9 \mathrm{kPa}$ and $\phi \approx 0.34$ at $300 \mathrm{~Hz}$. In view of these results, the authors concluded that the white matter anisotropy was significant but relatively small.

Employing the same methodology as [58], Testu et al. (2017) [60] examined the viscoelastic parameters of the in vivo human brain tissue along a range of frequencies of $15-50 \mathrm{~Hz}$. Previous studies reported rate-dependence of brain tissue as a monotonic shear modulus stiffening with increasing frequency. Nonetheless, Testu et al. suggested that this hypothesis could be assumed globally but found local discrepancies in which the trend was inverted. Specifically, a decrease of the loss modulus with frequency was obtained in the region surrounding the falx. Globally, the storage and loss moduli reported in this work were in the range of $0.8-1 \mathrm{kPa}$ and $0.25-0.4 \mathrm{kPa}$ for 15 $\mathrm{Hz}$ and $1.15-1.2 \mathrm{kPa}, 1.02-1.07 \mathrm{kPa}$ at $50 \mathrm{~Hz}$. Further improvements for the accuracy of the MRE technique were introduced by Johnson et al. (2016) [61] mainly focusing on two features: increased image spatial resolution and soft prior regularization (SPR) in the inversion procedure. SPR is a technique 
that allows the selection of the regions of interest prior to the calculation of the displacement fields. This way, the resultant shear stiffness will be more homogeneous and remain unaffected by the adjoining tissues (especially the cerebrospinal fluid, CSF). Huang et al. (2019) [62] employed a Hemholtz inversion algorithm to obtain the shear moduli of white and gray matter of 10 human volunteers, obtaining an average $\mu$ of $3.36 \mathrm{kPa}$ for the first tissue and $2.24 \mathrm{kPa}$ for the second. These values correspond to a frequency of excitation of $40 \mathrm{~Hz}$. Nonetheless, greater frequencies $(60 \mathrm{~Hz})$ led to an overall stiffening of the cerebrum and specially for the gray matter $(3.33 \mathrm{kPa})$.

A recent MRE-based study by Kolipaka et al. (2018) [63] aimed to determine the influence of pressure changes in the cerebrospinal fluid on the overall brain stiffness. Their study included both healthy patients and patients with idiophatic intracranial hypertension (IIH), which is a pathology characterised by raised CSF pressure values in the intracranial cavity. This last set of patients was determined to have higher average brain stiffnesses than the nonIIH group, demonstrating the long-term effect of stationary raised intracranial pressure values. Nonetheless, the authors attempted to find a correlation between brain stiffness and temporary CSF pressure changes resultant from lumbar puncture (CSF drainage). However, no CSF pressure-brain stiffness relation was found in this study, maybe due to physiological limitations in the procedure.

The latest MRE studies have come to demonstrate an aging effect in the mechanical properties of the brain. Hiscox et al. (2018) [55] studied the viscoelastic properties of different brain regions among 12 young and 12 older adults, obtaining a significant shear stiffness decrease in both the cerebrum and several subcortical gray matter structures. Viscosity, however, appears to remain unaltered with age suggesting that despite the composition of the tissue may be affected by time, the overall tissue microstructure is conserved. This finding is consistent with previous works on a greater population sample $[64,65]$ which predicted a shear stiffness decrease of a $0.8 \%$ per year. Other studies (Arani et al. (2015) [66]) reported this age influence to be highly regiondependent, finding peripherial brain areas to be more age-sensitive that inner structures.

Even though MRE has not led yet to a complete characterisation of the brain tissue, this technique is promising because of its application in vivo. Experimental in vitro studies have been the most common modus operandi up 
to date in order to capture brain tissue response under different loading conditions. However, the assumption that ex vivo brain samples do not change the mechanical properties of the living tissue if properly hydrated during testing appears to be progressively less plausible. Recent works, like the one by Weickenmeier et al. (2018) [67] have studied the mechanical differences between in vivo and ex vivo pig brains through MRE and obtained a pronounced stiffening in the non-living case of about $26-60 \%$ immediately after death and with an increasing tendency with post mortem time. The fact that this stiffening did not affect to all the brain regions to the same extent encouraged the authors to suggest that metabolic phenomena are responsible for the change in mechanical properties and they are more complex to predict that simple tissue dehydration.

Some of the results reported from the literature on the mechanical behaviour of the brain tissue as a whole, and of the gray and white matter are summarised in Table 2.3. Furthermore, various findings on other specific brain regions are summarised in Table 2.4 . 


\begin{tabular}{|c|c|c|c|c|}
\hline Region & Test type & Parameter & Value & Ref. \\
\hline Cerebrum & $\begin{array}{l}\text { Unconfined compression } \\
\text { Porcine in vitro DST } \\
\text { MRE } \\
\text { MRE+SPR } \\
\text { MRE } \\
\text { MRE+SPR } \\
\text { MRE } \\
\text { MRE }\end{array}$ & $\begin{array}{l}E_{0}(\mathrm{kPa}) \\
G(\mathrm{kPa}) \text { at } 0.1 \mathrm{~Hz} \\
G(\mathrm{kPa}) \text { at } 10 \mathrm{~Hz} \\
G(\mathrm{kPa}) \text { at } 260 \mathrm{~Hz} \\
\mu(\mathrm{kPa}) \\
\mu(\mathrm{kPa}) \\
\mu(\mathrm{kPa}) \\
\mu(\mathrm{kPa}) \\
\mu(\mathrm{kPa}) \text { at } 40 \mathrm{~Hz} \\
\mu(\mathrm{kPa}) \text { at } 60 \mathrm{~Hz} \\
G^{\prime}(\mathrm{kPa}) \text { at } 15 \mathrm{~Hz} \\
G^{\prime}(\mathrm{kPa}) \text { at } 50 \mathrm{~Hz} \\
G^{\prime \prime}(\mathrm{kPa}) \text { at } 15 \mathrm{~Hz} \\
G^{\prime \prime}(\mathrm{kPa}) \text { at } 50 \mathrm{~Hz}\end{array}$ & $\begin{array}{l}6.55 \\
0.50 \\
0.66 \\
1.25 \\
3.25 \pm 0.52 \\
3.20 \pm 0.24 \\
2.60 \pm 0.10 \\
2.95 \pm 0.17 \\
2.57 \pm 0.04 \\
3.27 \pm 0.05 \\
0.80-1.00 \\
1.15-1.20 \\
0.25-0.40 \\
1.02-1.07\end{array}$ & $\begin{array}{l}{[65]} \\
{[61]} \\
{[66]} \\
{[55]} \\
{[62]}\end{array}$ \\
\hline Grey matter & $\begin{array}{l}\text { MRE } \\
\text { MRE } \\
\text { MRE }\end{array}$ & $\begin{array}{l}G^{\prime}(\mathrm{kPa}) \text { at } 90 \mathrm{~Hz} \\
G^{\prime \prime}(\mathrm{kPa}) \text { at } 90 \mathrm{~Hz} \\
\mu(\mathrm{kPa}) \\
\mu(\mathrm{kPa}) \text { at } 40 \mathrm{~Hz} \\
\mu(\mathrm{kPa}) \text { at } 60 \mathrm{~Hz}\end{array}$ & $\begin{array}{l}3.10 \pm 0.10 \\
2.50 \pm 0.20 \\
2.22 \pm 0.35 \\
2.24 \pm 0.14 \\
3.33 \pm 0.14\end{array}$ & $\begin{array}{l}{[56]} \\
{[65]} \\
{[62]}\end{array}$ \\
\hline White matter & $\begin{array}{l}\text { Human in vitro DST } \\
\text { Bovine in vitro DST } \\
\text { Porcine in vitro } \\
\text { Porcine in vitro DST } \\
\text { Porcine ex vivo MRE }\end{array}$ & $\begin{array}{l}G^{\prime}(\mathrm{kPa}) \text { at } \mathrm{LF} \\
G^{\prime \prime}(\mathrm{kPa}) \text { at } \mathrm{LF} \\
G^{\prime}(\mathrm{kPa}) \text { at } 0.1 \mathrm{~Hz} \\
G^{\prime \prime}(\mathrm{kPa}) \text { at } 0.1 \mathrm{~Hz} \\
G^{\prime}(\mathrm{kPa}) \text { at } 0.1 \mathrm{~Hz} \\
G^{\prime \prime}(\mathrm{kPa}) \text { at } 0.1 \mathrm{~Hz} \\
G^{\prime}(\mathrm{kPa}) \text { at } 6310 \mathrm{~Hz} \\
G^{\prime \prime}(\mathrm{kPa}) \text { at } 6310 \mathrm{~Hz} \\
G_{\|}^{\prime}(\mathrm{kPa}) \text { at } 20 \mathrm{~Hz} \\
G_{\perp}^{\prime}(\mathrm{kPa}) \text { at } 20 \mathrm{~Hz} \\
G_{\|}^{\prime \prime}(\mathrm{kPa}) \text { at } 20 \mathrm{~Hz} \\
G_{\perp}^{\prime \prime}(\mathrm{kPa}) \text { at } 20 \mathrm{~Hz} \\
G_{\perp}(\mathrm{kPa}) \text { at } 100 \mathrm{~Hz} \\
\phi \text { at } 100 \mathrm{~Hz} \\
G_{\perp}(\mathrm{kPa}) \text { at } 300 \mathrm{~Hz} \\
\phi \text { at } 300 \mathrm{~Hz} \\
G^{\prime}(\mathrm{kPa}) \text { at } 90 \mathrm{~Hz} \\
G^{\prime \prime}(\mathrm{kPa}) \text { at } 90 \mathrm{~Hz} \\
\mu(\mathrm{kPa}) \text { at } 40 \mathrm{~Hz} \\
\mu(\mathrm{kPa}) \text { at } 60 \mathrm{~Hz}\end{array}$ & $\begin{array}{l}0.60-1.10 \\
0.35-0.60 \\
2.50 \\
0.85 \\
2.1 \\
0.40 \\
16.80 \\
18.70 \\
0.61 \pm 0.06 \\
0.54 \pm 0.08 \\
0.34 \pm 0.05 \\
0.30 \pm 0.05 \\
1.04 \pm 0.12 \\
0.27 \pm 0.09 \\
2.88 \pm 0.34 \\
0.34 \pm 0.13 \\
2.70 \pm 0.10 \\
2.50 \pm 0.20 \\
3.36 \pm 0.11 \\
3.85 \pm 0.12\end{array}$ & $\begin{array}{l}{[25]} \\
{[25]} \\
{[42]}\end{array}$ \\
\hline
\end{tabular}

Table 2.3: Some reported elastic properties of the cerebrum and its two main constituents from the literature. $\mu$ refers to the shear stiffness, $G$ to the shear modulus and $G^{\prime}, G^{\prime \prime}$ represent the storage and loss moduli, respectively. 


\begin{tabular}{|c|c|c|c|c|}
\hline Region & Test type & Parameter & Value $(\mathrm{kPa})$ & Ref. \\
\hline Thalamus & $\begin{array}{l}\mathrm{MRE}+\mathrm{SPR} \\
\mathrm{MRE}+\mathrm{SPR}\end{array}$ & $\begin{array}{l}\mu \\
\mu\end{array}$ & $\begin{array}{l}3.82 \pm 0.23 \\
3.35 \pm 0.24\end{array}$ & $\begin{array}{l}{[61]} \\
{[55]}\end{array}$ \\
\hline Amygdala & $\begin{array}{l}\text { MRE+SPR } \\
\text { MRE+SPR }\end{array}$ & $\begin{array}{l}\mu \\
\mu\end{array}$ & $\begin{array}{l}3.83 \pm 0.35 \\
3.60 \pm 0.30\end{array}$ & $\begin{array}{l}{[61]} \\
{[55]}\end{array}$ \\
\hline Hippocampus & $\begin{array}{l}\text { MRE+SPR } \\
\text { MRE+SPR }\end{array}$ & $\begin{array}{l}\mu \\
\mu\end{array}$ & $\begin{array}{l}3.35 \pm 0.35 \\
2.89 \pm 0.32\end{array}$ & $\begin{array}{l}{[61]} \\
{[55]}\end{array}$ \\
\hline Caudate & $\begin{array}{l}\mathrm{MRE}+\mathrm{SPR} \\
\mathrm{MRE}+\mathrm{SPR}\end{array}$ & $\begin{array}{l}\mu \\
\mu\end{array}$ & $\begin{array}{l}3.75 \pm 0.27 \\
2.92 \pm 0.23\end{array}$ & $\begin{array}{l}{[61]} \\
{[55]}\end{array}$ \\
\hline Putamen & $\begin{array}{l}\mathrm{MRE}+\mathrm{SPR} \\
\mathrm{MRE}+\mathrm{SPR}\end{array}$ & $\begin{array}{l}\mu \\
\mu\end{array}$ & $\begin{array}{l}3.87 \pm 0.19 \\
3.76 \pm 0.15\end{array}$ & $\begin{array}{l}{[61]} \\
{[55]}\end{array}$ \\
\hline Pallidum & $\begin{array}{l}\text { MRE+SPR } \\
\text { MRE+SPR }\end{array}$ & $\begin{array}{l}\mu \\
\mu\end{array}$ & $\begin{array}{l}3.84 \pm 0.26 \\
3.59 \pm 0.26\end{array}$ & $\begin{array}{l}{[61]} \\
{[55]}\end{array}$ \\
\hline Frontal lobes & $\begin{array}{l}\text { MRE } \\
\text { MRE }\end{array}$ & $\begin{array}{l}\mu \\
\mu\end{array}$ & $\begin{array}{l}3.20 \pm 0.73 \\
2.70 \pm 0.20\end{array}$ & $\begin{array}{l}{[65]} \\
{[66]}\end{array}$ \\
\hline Occipital lobes & $\begin{array}{l}\text { MRE } \\
\text { MRE }\end{array}$ & $\begin{array}{l}\mu \\
\mu\end{array}$ & $\begin{array}{l}2.70 \pm 0.57 \\
2.80 \pm 0.30\end{array}$ & $\begin{array}{l}{[65]} \\
{[66]}\end{array}$ \\
\hline Parietal lobes & MRE & $\mu$ & $2.60 \pm 0.20$ & {$[66]$} \\
\hline Temporal lobes & MRE & $\mu$ & $2.80 \pm 0.20$ & {$[66]$} \\
\hline Deep GM/WM & $\begin{array}{l}\text { MRE } \\
\text { MRE }\end{array}$ & $\begin{array}{l}\mu \\
\mu\end{array}$ & $\begin{array}{l}4.45 \pm 0.74 \\
3.00 \pm 0.20\end{array}$ & $\begin{array}{l}{[65]} \\
{[66]}\end{array}$ \\
\hline Cerebellum & MRE & $\mu$ & $2.20 \pm 0.20$ & {$[66]$} \\
\hline Sensory-motor & MRE & $\mu$ & $2.80 \pm 0.30$ & {$[66]$} \\
\hline
\end{tabular}

Table 2.4: Experimentally obtained elastic properties from other brain regions. $\mu$ refers to the shear stiffness, $G$ to the shear modulus and $G^{\prime}, G^{\prime \prime}$ represent the storage and loss moduli, respectively. 


\subsubsection{Constitutive modeling of brain tissue}

Proper modeling of the mechanical behabiour of brain tissue still remains a challenge up to date. An inherent feature of brain that makes it dissimilar to the rest of soft living tissues is that its structure is not composed by collagen and elastin fibers [68]. Therefore, common constitutive models employed for other biological tissues cannot provide an adequate prediction of the brain's behaviour. Pioneer models on the mechanical response of brain tissue accounted for linear elasticity approaches, while the increase of available experimental data allowed to capture its time-dependent behaviour by means of linear viscoelastic laws. Experimental studies pointed out that brain tissue behaves more compliantly when strains above the elastic limit are applied. For this reason, hyperelastic material formulations acceptably resemble experimental curves. Nonetheless, brain tissue was further considered as highly strain-rate dependent and also strain-level dependent, which requires complementing the hyperelastic laws with an adequate stress relaxation formulation. Another issue in the modeling step is whether to consider the whole brain as a homogeneus structure or distinguish between its constituents. Some works modeled white and gray matter separately, each as a homogeneous tissue with different material parameters. Other studies, however, aimed to capture the anisotropy of white matter by incorporating information from DTI images, either by means of a probabilistic function of axonal distribution inside the white matter lattice or with a detailed modeling of the axonal pattern. Another existing modeling proposal which still remains under debate is the consideration of the biphasic solid-fluid nature of brain tissue, which has been addressed in some studies by means of porosity laws.

The first studies on brain response by Galford and McElhaney (1970) [30] and Shuck and Advani (1972) [32] modeled the cerebrum tissue (including both gray ang white matter) as an isotropic linear viscoelastic material by means of a Maxwell-Kelvin four-parameter-fluid model as schematised in Fig. $2.5 \mathrm{a})$ and b). 

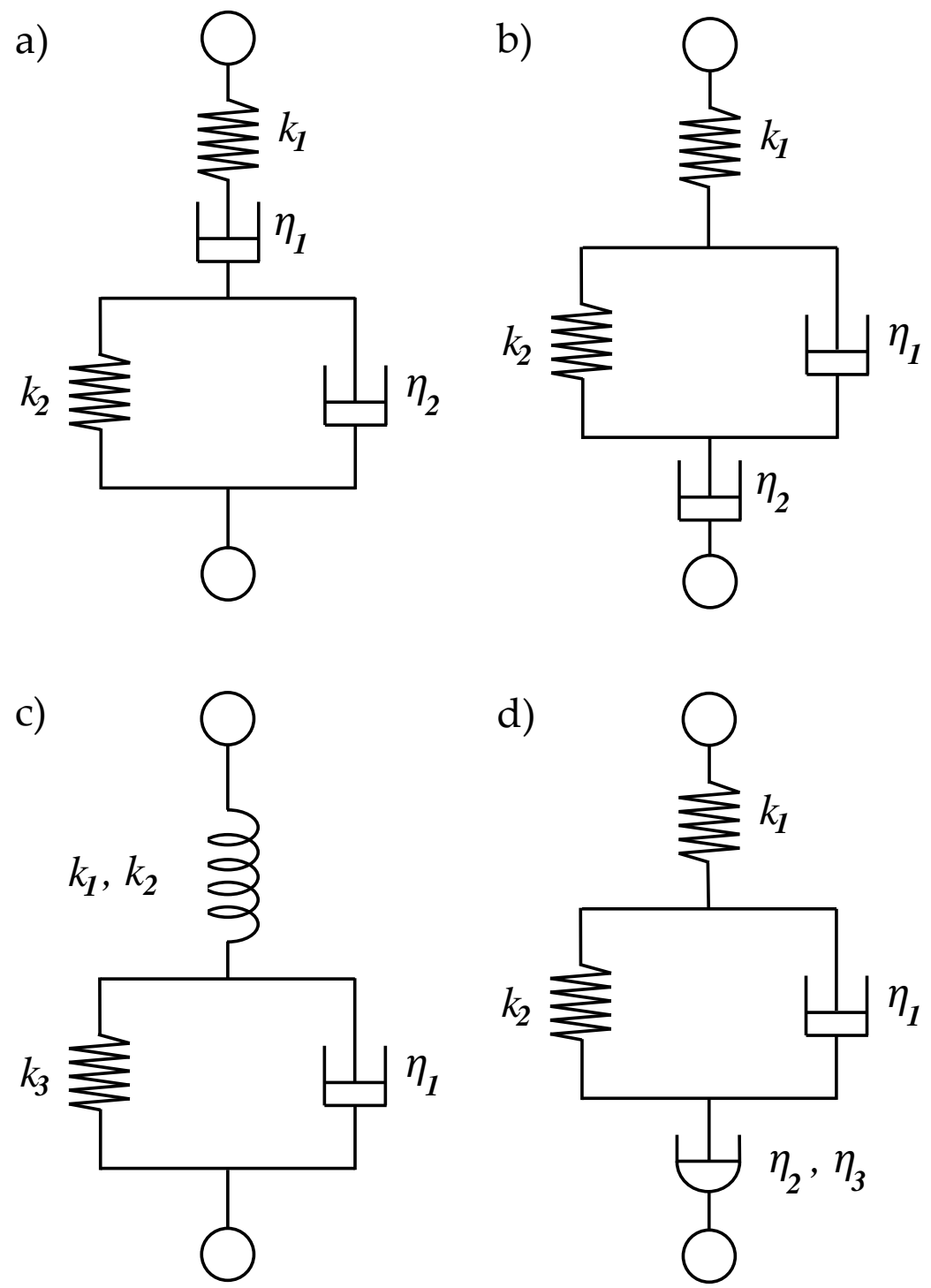

Figure 2.5: Four-element rheological models proposed by Galford and McElhaney [30] (a) and Shuck and Advani [32] (b). Three-element solid (c) and four-element fluid (d) from Pamidi and Advani [69]. 
Posteriorly, Pamidi and Advani (1978) [69] aimed to characterise the behaviour of brain tissue by means of spring-dashpot models (for the viscoelastic behaviour) and a Mooney material function accounting for rate dependencies. It is relevant to mention that the outcome constitutive law is appliable only for uniaxial stress conditions. The simplified viscoelastic models are illustrated in Fig. 2.5 and constitute a nonlinear three-parameter solid with a quadratic spring (Fig. 2.5 c) and a four-parameter fluid like the one employed by Shuck and Advani [32] but with a quadratic dashpot (Fig. 2.5 d). Creep tests performed by Galford and McElhaney [30] were employed to calibrate the constants of each model. Stress prediction was validated against Estes and McElhaney [31] unconfined uniaxial compression tests at different strain rates, through the stress equation derived from the Mooney material model:

$$
\begin{aligned}
\sigma_{11}= & \underbrace{2\left[C_{1} \lambda^{2}-\frac{C_{2}}{\lambda^{2}}\right]}_{\text {elastic stress }}+\underbrace{2\left[C_{2} \lambda-\frac{C_{1}}{\lambda}\right]}_{\text {'elastic' hydrostatic pressure }}+ \\
& +\underbrace{2 B_{1} \dot{\lambda}\left[\frac{1}{\lambda}-4\right]}_{\text {viscous stress }}-\underbrace{4 B_{1} \dot{\lambda}\left[1-\frac{1}{\left.2 \lambda^{3}\right]}\right.}_{\text {'viscous' hydrostatic pressure }}
\end{aligned}
$$

The experimental results by Estes and McElhaney [31] were also employed by Mendis et al. (1995) [70] to develop a constitutive law for brain modeling applicable to large deformations. It is taken as an incompressible hyperelastic material whose mechanical behaviour is governed by a strain energy density function (SEDF) expressed in terms of the Mooney-Rivlin equation (Eq. 2.3):

$$
W=\sum_{i=0}^{N} \sum_{j=0}^{N} C_{i j}\left(I_{1}-3\right)^{i}\left(I_{2}-3\right)^{j}
$$

being $I_{1}$ and $I_{2}$ the first and second invariants of the Cauchy-Green strain tensor. The contribution of the third invariant is removed from the SEDF due to the assumed incompressibility of the material. Particularising Eg 2.3 to the first-order case $(\mathrm{N}=1), U$ is reduced to the expression

$$
W=C_{10}\left(I_{1}-3\right)+C_{01}\left(I_{2}-3\right)
$$


The hyperelastic constants $C_{10}$ and $C_{01}$ can be related to other elastic constants under the assumption of small strains:

$$
\mu=2\left(C_{10}+C_{01}\right) \quad E=6\left(C_{10}+C_{01}\right)
$$

Energy dissipation as a result of the viscoelastic nature of the brain can be implemented in the constitutive model by means of a time-relaxation law of the elastic constants (in this case, a Prony series):

$$
C(t)=C_{0}-\sum_{i=1}^{N} C_{i}\left(1-e^{-\frac{t}{\tau_{i}}}\right)
$$

Mendis et al. extracted the numerical values for the constants of Eq. 2.6 particularised to brain tissue using two terms of the Prony series, which were derived from the results of Estes and McElhaney from different loading rates:

$$
C_{10}(t)=0.9 C_{01}(t)=620.5+1930 e^{-t / 0.008}+1103 e^{-t / 0.15}
$$

Eq. 2.7 has been posteriorly applied to human head finite element models by other authors, but Miller and Chinzei [33] criticised the assumption taken in [70] that the experimental test data employed corresponded to the quasistatic range. Instead, they proposed and validated an hyperviscoelastic constitutive model for swine brain under strain rates below $1 \mathrm{~s}^{-1}$. The material formulation was the same as the proposed in [70] but introducing four hyperelastic constants in the Mooney-Rivlin equation $\left(C_{10}=C_{01}=263 \mathrm{~Pa}\right.$ and $C_{20}=C_{02}=491 \mathrm{~Pa}$ ).

Other works have approximated the non-linear elastic response by means of an hyperelastic Ogden model, whose SEDF expression is defined in Eq. 2.8.

$$
W(\alpha)=\sum_{k} \frac{\mu_{k}}{\alpha_{k}}\left(\lambda_{1}^{\alpha_{k}}+\lambda_{2}^{\alpha_{k}}+\lambda_{3}^{\alpha_{k}}-3\right)
$$

being $\mu_{k}$ and $\alpha_{k}$ the material properties and $\lambda_{i}$ the principal shear strain ratios. For instance, a one-parameter Ogden model was proposed by Budday et 
al. [71] to fit their experimental results on different brain regions of porcine samples. Fig. 2.6 illustrates these results in compression and shear (the constitutive Ogden model and the experimental average responses overlap).
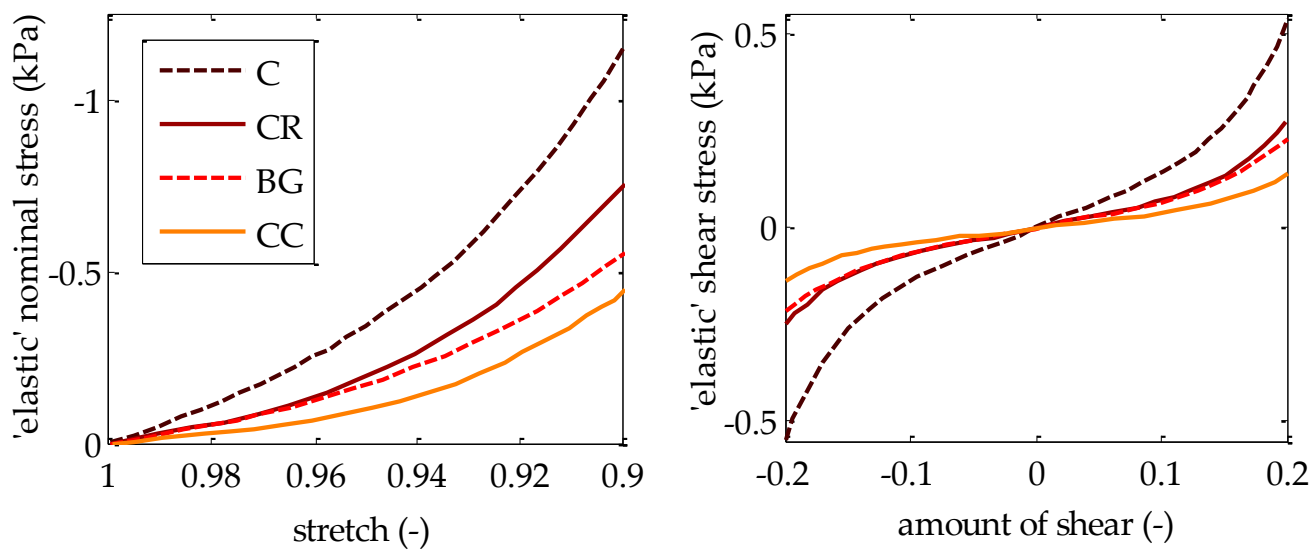

Figure 2.6: One-parameter Ogden models fitting the experimental curves from Budday et al. [71] for different brain regions. $\mathrm{C}=$ cortex, $\mathrm{CR}=$ corona radiata, $\mathrm{BG}=$ basal ganglia, $\mathrm{CC}=$ corpus callosum.

Analogously to the Mooney-Rivlin model, in the case of quasi-infinitesimal strains the relation in Eq. 2.9 can be derived.

$$
G=\sum_{k} \frac{\mu_{k} \alpha_{k}}{2}
$$

Brands et al. (2004) [72] proposed a constitutive law for the homogenised brain tissue applicable to large strains $(20 \%)$ and strain rates $\left(8 \mathrm{~s}^{-1}\right)$. Nonetheless, experimental data employed for model calibration did not allow the extrapolation of the constitutive model to higher strain rates which are normally associated to impact-induced head injury.

Other large-strain constitutive formulations for brain tissue have been proposed in some works such as Hrapko et al. [28] and Prevost et al. [46]. The latter proposed a non-linear visco-hyperelastic model whose rheological scheme is depicted in Fig. 2.7, and was numerically validated against unconfined compression and tension experiments on porcine samples. Elastic (A) and 
viscoelastic (B-E) elements are coupled to model the instantaneous and the dissipative response, respectively. The first (instantaneous) contribution accounts for a deviatoric (shear-dependent) and an hydrostatic (compressibilitydependent) term. The formulation of the deviatoric component, $\mathbf{T}_{\mathrm{d}}$, is based on an eight chain model for elastomeric materials, which consideres a network of not-aligned macromolecular chains able to move with respect to each other at the same time that they deform until a certain stretch limit $\lambda_{\mathrm{L}}$. The hydrostatic term, $\mathbf{T}_{\mathrm{h}}$, relies on the material bulk modulus and its incompressible fraction (which is asumed to be the $80 \%$ ). Element (B) from the scheme represents the short-term non-linear viscoelastic response and depends on two material properties: a strength parameter $\sigma_{0}$ and a rate sensitivity exponent $n$. The remaining elements constitute the viscoelastic backstress network, being $(\mathrm{C})$ and $(\mathrm{E})$ functions of the short-term $\left(G_{0}\right)$ and the long-term $\left(G_{\infty}\right)$ shear moduli of the material. Finally, element (D) is the relaxation term of the system and depends of the viscosity $(\eta)$ of the material.

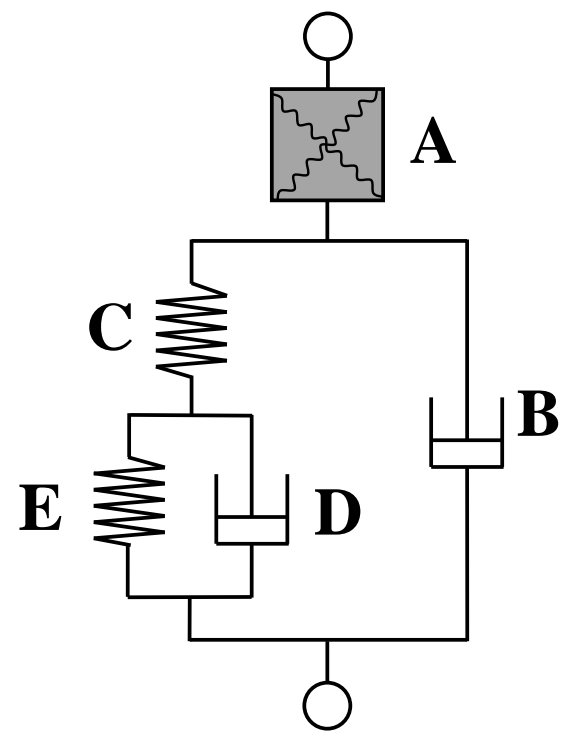

Figure 2.7: Scheme of the non-linear visco-hyperelastic material model proposed by Prevost et al. [46].

A recent approach by Haldar and Pal (2018) [73] formulated a model for 
the anisotropic behaviour of brain tissue based on experimental tests from the literature of brain in tension (Miller and Chinzei [33]), shear and unconfined compression (Hrapko et al. [28]), relaxation (Tamura et al. [74]) with the purpose of developing a constitutive law able to capture different loading/unloading conditions. Brain tissue is modeled in 2D as an homogeneous matrix reinforced with fibrous tubes in different directions. The constitutive model assumes that the total free energy of the system has five separated contributions, stated in Eq. 2.10.

$$
\begin{aligned}
\rho_{0} \Psi & \left(\mathbf{C}, \mathbf{C}^{f}, \mathbf{C}_{\nu \alpha}, \mathbf{C}_{\nu \beta}^{f}\right)=\underbrace{\rho_{0} \Psi_{\text {vol }}(J)}_{\text {volumetric energy }}+\underbrace{\rho_{0} \Psi_{\infty}^{m}(\overline{\mathbf{C}})}_{\text {elastic matrix energy }}+ \\
+ & \underbrace{\sum_{f} \rho_{0} \Psi_{\infty}^{f}\left(\mathbf{C}^{f}\right)}_{\text {fibers elastic energy }}+\underbrace{\sum_{\alpha} \rho_{0} \Psi_{\alpha}^{m}\left(\overline{\mathbf{C}}, \mathbf{C}_{\nu \alpha}\right)}_{\text {viscoelastic matrix energy }}+\underbrace{\sum_{f} \sum_{\beta} \rho_{0} \Psi_{\beta}^{f}\left(\mathbf{C}^{f}, \mathbf{C}_{\nu \beta}^{f}\right)}_{\text {viscoelastic fibers energy }}
\end{aligned}
$$

Volumetric behaviour:

$$
\rho_{0} \Psi_{v o l}(J)=\frac{1}{2} K[J-1]^{2}
$$

Isochoric elastic law for the matrix: an Ogden strain energy potential is assumed (Eq. 2.12).

$$
\rho_{0} \Psi_{\infty}^{m}(\overline{\mathbf{C}})=\rho_{0} \Psi_{\infty}^{m}\left(\overline{\lambda_{1}}, \overline{\lambda_{2}}, \overline{\lambda_{3}}\right)=\sum_{p=1}^{N} \frac{\mu_{p}}{\alpha_{p}}\left({\overline{\lambda_{1}}}^{\alpha_{p}},{\overline{\lambda_{2}}}^{\alpha_{p}},{\overline{\lambda_{3}}}^{\alpha_{p}}-3\right)
$$

Isochoric elastic law for fibers: it is assumed that fibers only work under tension and therefore compressive strains are discarded by means of the operator \langle\rangle . The energy potential is estimated as a polynomic expression (Eq. $2.13)$.

$$
\rho_{0} \Psi_{\infty}^{f}\left(\mathbf{C}^{f}\right)=\frac{C_{r f}}{2 r}\left\langle I_{4 f}-1\right\rangle^{r}+\frac{D_{s f}}{2 s}\left\langle I_{4 f}-1\right\rangle^{s}
$$


Viscoelastic components: a neo-Hookean form is assumed for the matrix (Eq. 2.14) and a quadratic relation for the fibers (Eq. 2.15).

$$
\begin{gathered}
\rho_{0} \Psi_{\alpha}^{m}\left(\overline{\mathbf{C}}, \mathbf{C}_{\nu \alpha}\right)=\frac{\mu_{\nu \alpha}}{2}\left[\overline{\mathbf{C}}: \mathbf{C}_{\nu \alpha}^{-1}-3\right] \\
\rho_{0} \Psi_{\beta}^{f}\left(\mathbf{C}^{f}, \mathbf{C}_{\nu \beta}^{f}\right)=\frac{\mu_{\nu \beta}}{4}\left\langle\mathbf{C}^{f}:\left[\mathbf{C}_{\nu \beta}^{f}\right]-1\right\rangle^{2}
\end{gathered}
$$

Labus and Puttlitz [50] extended the Ogden hyperelastic model to account for the axon-reinforcements to characterise the anisotropy of white matter. To that purpose, the distribution parameter of the axons $\kappa$ (Eq. 2.16) and the axonal volume fraction $f_{a}$.

$$
\kappa=\frac{1}{4} \int_{0}^{\pi} \rho(\theta) \sin ^{3}(\theta) \mathrm{d} \theta
$$

Therefore, the resulting constitutive law in terms of the SEDF takes the form of Eq. 2.17.

$$
\begin{aligned}
& W=\frac{2 \mu}{\alpha^{2}}\left(\lambda_{1}^{\alpha}+\lambda_{2}^{\alpha}+\lambda_{3}^{\alpha}-3\right)+ \\
& +\left(k^{\prime} f_{a}+k_{0}\right) \frac{2 \mu}{\alpha^{2}}\left[\left(\kappa I_{1}+(1-3 \kappa) I_{4}\right)^{\frac{\alpha}{2}}+2\left(\kappa I_{1}+(1-3 \kappa) I_{4}\right)^{-\frac{\alpha}{4}}-3\right]
\end{aligned}
$$

Poroviscoelastic constitutive models have also been proposed in the literature for the representation of the behaviour of human brain tissue. Mehrabian and Abousleiman (2011) [75] presented the constitutive equations for isotropic poroviscoelastic and anisotropic poroviscoelastic materials with the expressions from Eq. 2.18 and 2.19, respectively:

$$
\sigma_{i j}-\delta_{i j} \alpha p=2 G \varepsilon_{i j}+\frac{3 K-2 G}{3} \delta_{i j} \varepsilon
$$




$$
\begin{gathered}
\sigma_{i j}-\delta_{i j} \alpha(t) \otimes p(t)=2 G(t) \otimes \varepsilon_{i j}(t)+\frac{3 K-2 G}{3} \otimes \varepsilon_{i j}(t) \\
G(t) \otimes \varepsilon_{i j}(t)=\int_{0}^{t} G(t-\tau) \mathrm{d} \varepsilon(\tau)
\end{gathered}
$$

being $\sigma_{i j}$ the stress tensor, $\varepsilon_{i j}$ the strain tensor, $\delta_{i j}$ the Kronecker delta, $K$ the material bulk modulus and $G$ the shear modulus.

Forte et al. (2017) [48] attempted to capture the CSF-immersed brain tissue by means of a byphasic poro-visco-hyperelastic model for both gray and white matter. The solid phase is stated as a hyper-viscoelastic material following a first-order Ogden law complemented with a Prony series for the time dependence of the elastic modulus. The fluid phase (CSF) was assumed to be a water-like material whose flow along the matrix tissue is defined by Darcy's equation:

$$
n \boldsymbol{v}=-\frac{k}{\gamma_{w}}\left(\nabla p-\rho_{w} \boldsymbol{g}\right)
$$

with $n$ as brain matrix porosity, $\boldsymbol{v}$ the flow velocity, $\nabla p$ the pressure gradient and $k$ the medium conductivity. Swelling was permitted in their FE brain model.

\subsection{Other structures of the head}

As stated at the beginning of this chapter, the human head is comprised of many structures with different tissue typologies, all playing a determinant role in the protection of intracranial contents, hydration or blood irrigation. Having extensively described the main components of the head involved in impact behaviour and injury (skull and brain), some other relevant head constituents will be briefly addressed in this section.

\subsubsection{The meninges}

The meninges is a membranous complex placed between the skull and the central nervous system (CNS) whose function is to protect the brain and damp 
its intracranial displacements [76, 77], and it is also hypothesised that plays a role in fetal brain development [78]. The meninges act as an active connective tissue that enhances immunological and homeostatic processes in the CNS [79]. Three layers conform this structure: the dura mater, arachnoid mater and pia mater (see Fig. 2.8). Each of them will be discussed in this section.

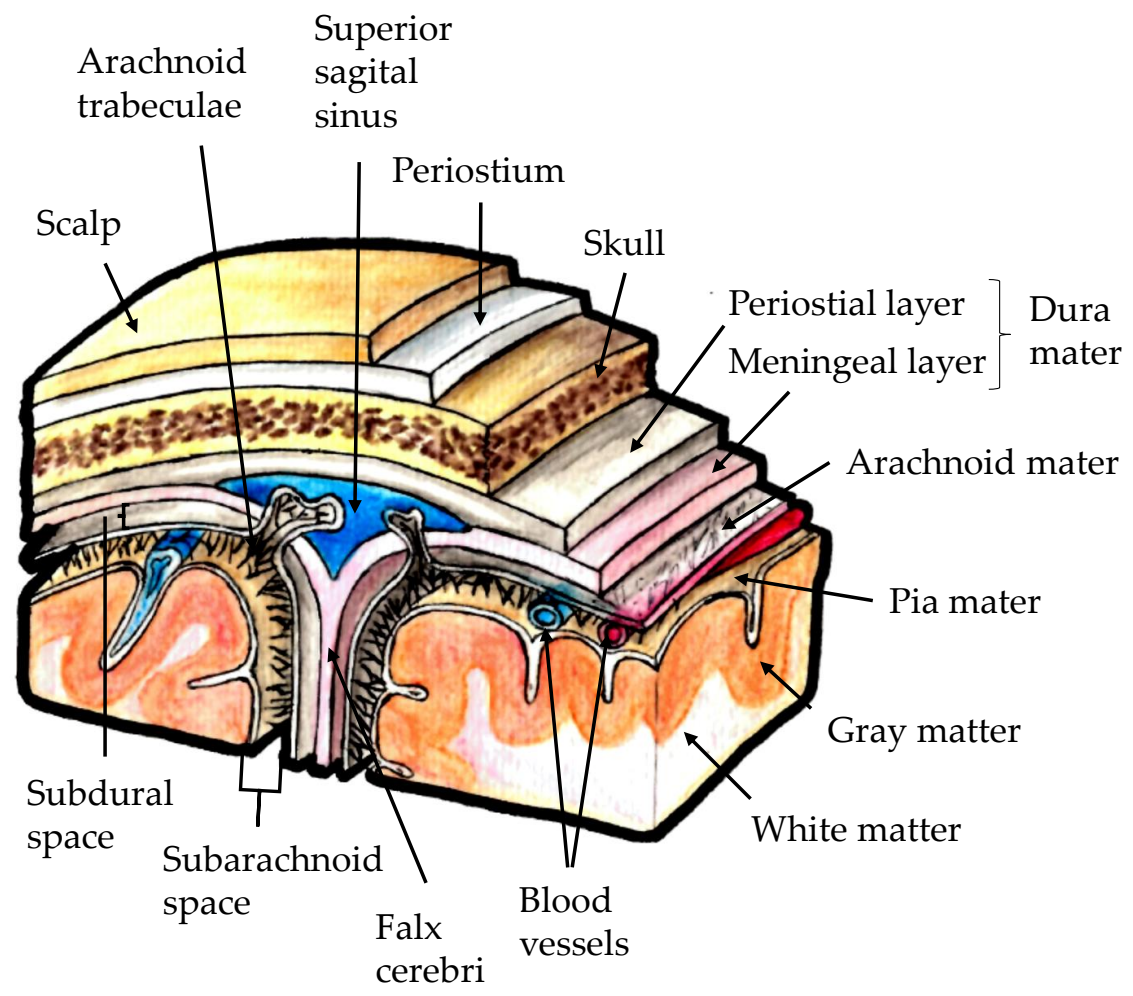

Figure 2.8: Scheme of the meninges membranous complex.

\section{Dura mater}

Structurally, cranial dura mater consists of two adhered layers: one of them is in contact with the skull bones while the other acts as a junction between the first and the arachnoid layer [80]. Histologically, it is a collagenous membrane in which several blood and lymphatic vessels are embedded [79]. The distribution of elastin and wavy collagen fibers makes this membrane relatively isotropic [81] even though more recent studies suggest the existence of highly 
aligned fiber regions in the temporal lobes [82], therefore biasing the behaviour of duramater towards an anisotropic material.

Most of the human head finite element models which explicitly model dura mater assume a linear elastic behaviour. Nonetheless, a recent experimental study by De Kegel et al. (2018) [83] demonstrated that the optimal constitutive model for the dura mater was a Gasser-Ogden-Holzapfel (GOH) model. Assuming isotropic behaviour, this constitutive law is governed by three material parameters $\left(C_{10}, k 1\right.$ and $\left.k_{2}\right)$, whose expression for the strain energy potential is the following:

$$
\Psi\left(\mathrm{I}_{1}\right)=C_{10}\left(\mathrm{I}_{1}-3\right)+\frac{k_{1}}{k_{2}}\left\{\exp \left[k_{2}\left(\frac{1}{3} \mathrm{I}_{1}-1\right)^{2}\right]-1\right\}
$$

\section{Arachnoid and pia mater (leptomeninges)}

The arachnoid mater is in contact with the dura at its external surface and adjoins pia mater, membrane which recovers the CNS surface. The structure of the arachnoid layer is rather heterogeneous as the subarachnoid space comprises trabecular structures which enhance the fluid transfer with the pia mater and also holds the space in which cerebrospinal fluid (CSF) circulates [84]. The pia mater is a thin connective tissue which surrounds the brain along its entire surface, carefully covering the sulci and gyri [80] that constitute its folded appearance.

\subsubsection{Falx and tentorium}

The falx cerebri is a tough membrane which arises from the dura mater and separates the cerebral hemispheres. Many of the head sinuses are housed in this anatomical structure. In fact, the upper limit of this dural fold is the superior sagittal sinus (SSS), whereas the lower limit is a free edge [85]. A recent study by Walsh et al. (2018) [86] of uniaxial testing on porcine dura mater samples found a significantly greater stiffness of the dural layer in the SSS region (increase of almost a $100 \%$ with respect to the frontal, parietal, occipital and temporal regions). This finding is relevant for the development of head models as it is likely to constrain the deformation and displacement of the SSS-falx complex during brain movement. 


\subsubsection{Blood vessels}

As previously stated, the human head is a highly vascularised structure as all of its constituents require continuous blood supply. Morphology and size of the blood vessels in the human head vary according to its location and function. For instance, the largest vessels, which are the carotid arteries and vertebral arteries, lead the irrigation of the brain, face and scalp. These subdivide in shorter and thinner arteries along the brain lattice from the interior to the periphery.

The same trend occurs in the opposite side of the blood flow. Small veins irrigating the face and scalp fuse in larger vessels (mainly the jugular veins). The blood exchange for the brain and skull is performed by the sinuses, intracranial cavities in which the blood is collected for its drainage. For this reason, there is a large number of vessels traversing the dura mater in order to allow the blood supply of the sinuses. This last vascular complex is conformed by the so-called bridging veins.

\subsubsection{Scalp}

Scalp is a five-layered soft tissue covering the cranial vault. The most internal layer is the pericranium, which is a prolongation of the periosteum. It is followed by a highly vascularised layer of loose connective tissue that enables the movement of the three outer layers. On top of it lies the aponeurosis, a musculo-fibrous layer that controls the facial movements. This layer is covered by a dense and cavernous connective tissue that also holds blood vessels and neural transmissions. Finally, the most external constituent of the scalp is the skin, which is highly irrigated and contains the sensitive terminations.

Therefore, it can be stated that the scalp is a highly complex heterogeneous tissue. Mechanical tests on human (Melvin et al. [87]) and monkey scalp (Galford and McElhaney [30]) were performed in the 1970s, but little information about this tissue can be found in the recent literature. It has been addressed in some studies, focusing on its behaviour under tension (Raposio and Nordström (1997) [88], Falland-Cheung et al. (2018) [89]) most of them obtaining a four-to-five times greater stiffness than regular skin [90]. 


\subsection{Head Injury Criteria}

\subsubsection{Introduction to head injuries}

Head injuries constitute a wide field, making it difficult to obtain well-established categories. The most common head injury typologies are classified as follows [91-93]:

- By mechanism of injury. Two categories are distinguished according to this criterion: open and closed injuries. The first, also known as penetrating injuries, account for the cases in which there is a skull fracture. Closed injuries occur in the absence of skull fracture and can be caused by contact loadings (therefore they become blunt injuries) or without direct contact.

- By morphology. This group is subdivided in three categories: skull fracture, focal injuries and diffuse injuries.

- Skull fractures: According to the topology of the cracks they can be subdivided as cranial vault fractures or basilar fractures. Basilar fractures (BSF) are defined as any fracture that begins or propagates to the base of the skull and their normal consequences are the leakage of cerebrospinal fluid (CSF) or haemorrhages and if located close to the foramen magnum they can even result in instantaneous death [94]. Fractures in the cranial vault can lead to different injury outcomes depending on their pattern, location and severity. They can be further classified as linear, depressed or stellated although frequently these fracture patterns can appear together.

- Focal injuries: caused by contact loads and resulting in different outcomes like contusions, subdural and epidural hematoma. These imply damage to the vascular intracranial tissues, and their classification is normally related to the location of the teared blood vessels or the location of the blood leakage. When the haemorrhage takes place between the head membranes it remains normally isolated and contained by them.

- Contusions: this injury type results from direct mechanical loading to the brain immediately after an impact event. The clinical manifestation is normally damage in the vascular tissue embedded in the subarachnoidal space and in the cortical surface of the brain. When 
the contusion is located at the impact site it is identified as a coup injury, whereas if it is located at the site opposite to the loading it is named contrecoup injury. These two lesion types also result in different injury outcomes. Specifically, contrecoup mechanisms and their injury potential are not fully understood yet, although there is certain belief that cavitation phenomena are likely to occur under these conditions.

- Subdural Hematoma (SDH): It is caused by the rupture of the bridging veins that connect the cerebral cortex with the sinuses [92] and is normally associated with severe degrees of TBI. Its location is set between the dura matter and the arachnoid when they separate as a result of a trauma event [95]. SDH may affect the brain in different ways: creating and increasing pressure loads on the cerebral surface, brain swelling and even triggering mechanisms of Diffuse Axonal Injury (DAI).

- Epidural Hematoma (EDH): consists on localised bleeding in the interface skull-dura matter, causing the detachment of the membrane of the inner skull table. Commonly its prognosis is more favorable than the one for SDH.

- Diffuse injuries: they appear when the brain is subjected to accelerations or decelerations, that can arise from contact or non-contact loading of the head. DAI and concussion are the principal manifestations of this kind of injury outcome.

- Concussion: it refers to a post-traumatic outcome (normally recoverable) which involves loss of conciousness, confusion and amnesia, among other less-common symptoms [96]. Nonetheless, if permanent damage is imparted to the brain, long-term psychological and physical consequences may arise.

- Diffuse Axonal Injury (DAI): It is one of the most common manifestations of TBI, which arises from the stretching and tearing of the white matter axons during short-pulse head accelerations [97].

Despite its different mechanisms, it is frequent that both kinds of injuries (focal and diffuse) appear simultaneously [98]. 
- By severity. According to this classification, injuries can range from mild to severe and are often labelled with a score according to the different existing injury scales. [99]

A scheme of the global classification of head injuries is shown in Fig. 2.9.

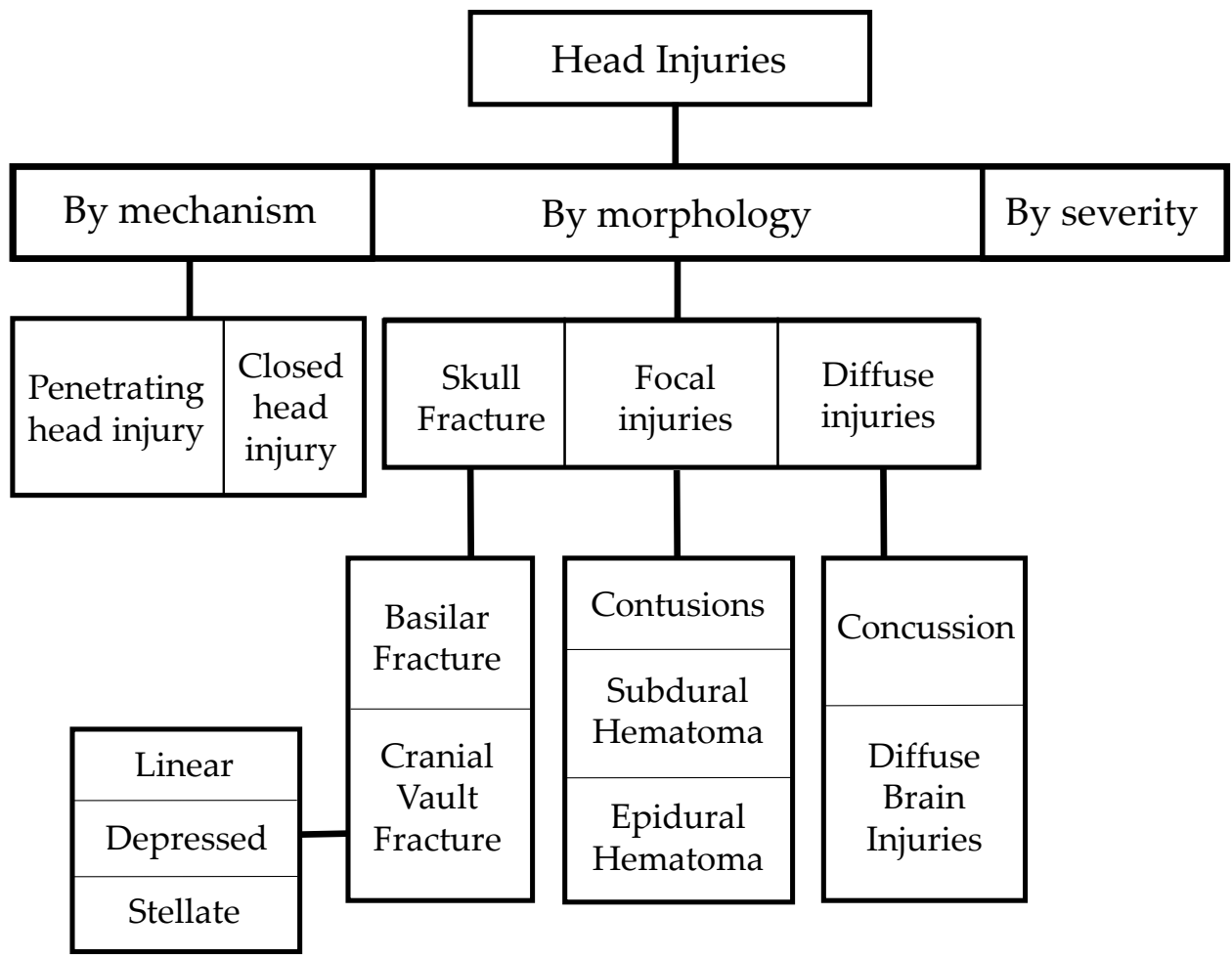

Figure 2.9: Classical classification of head injuries

This thesis will focus on Traumatic Brain Injury (TBI) which is the name given to any brain lession which occurs as a result of an external force on the head. Knowledge of the development of head injuries in terms of mechanical phenomena allows the prediction of lesions by means of several techniques like experimental testing with surrogates, or finite element analysis (FEA). Some of the most relevant findings in this field are summarised in the following pages. 


\subsubsection{Head injury criteria}

\section{Pioneering criteria}

One of the first studies providing head injury tolerance curves was performed by Gurdjian et al. [100] as a compendium of previous works [101, 102]. Impact experimental data from both anesthetized dogs [101] and human post-mortem subjects were gathered to set the frontiere between severe injury outcome and lower injury risk levels. In the tests involving dogs, impact conditions were applied by means of pressure pulses of different peak magnitudes and durations. They observed that more severe concussions were found either when the peak pressure was high or when the pulse width was wider. From this basis, they drew an intracranial pressure-time tolerance curve, whose extrapolation to human head injury prediction was not established due to anatomical differences between humans and dogs. Their second tolerance curve was accelerationbased and obtained from experimental impact tests on PMHS. The threshold outcome to determine severe injury was chosen to be the observation of linear skull fractures. The resulting curve, which is the basis of the well-known Wayne State Tolerance curve (WSTC, Fig.2.10), showed the asymptotical tendency that will be found in all posterior published works on human tolerance on translational acceleration.

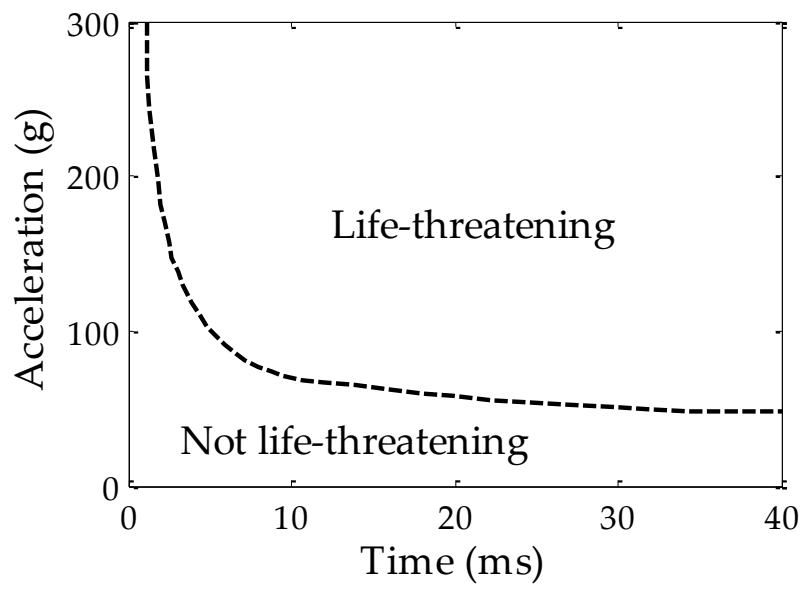

Figure 2.10: Wayne State Tolerance curve.

According to this injury prediction metric, the human head can withstand 
great acceleration values (around $250 \mathrm{~g}$ ) if pulse durations are short (lower than $2 \mathrm{~ms}$ ). Analogously, longer duration pulses can be sustained without severe concussion if the peak acceleration value decreases. This tolerance curve, however, was developed from impacts on the frontal site and therefore the prediction of injuries sustained in other regions is limited due to the inherent differences in skull bone tolerance.

Based on the principles of the WSTC, Gadd (1966) [103] proposed a Severity Index, taking into account the deceleration history of the head, which is analytically characterised as an exponential law whose weight factor is suggested to be 2.5. The general expression of the Gadd Severity Index is shown in Eq. 2.22.

$$
\mathrm{GSI}=\int_{0}^{\tau} \mathrm{a}(\mathrm{t})^{2.5} \mathrm{dt}
$$

A threshold value of GSI=1000 was proposed to define the transition between the life threatening and the non-threatening events.

Posteriorly, Versace (1971) [104] developed a new injury metric based on the aforementioned criteria, relating injury risk to the translational acceleration history. This metric, the Head Injury Criterion (HIC), was then adopted by the National Highway Traffic Safety Administration (NHTSA) [105] as a certification measure for the automotive and aeronautic safety industry [106]. Its analytical definition is given by:

$$
\mathrm{HIC}=\left\{\left[\frac{1}{\left(\mathrm{t}_{2}-\mathrm{t}_{1}\right)} \int_{\mathrm{t}_{1}}^{\mathrm{t}_{2}} \mathrm{a}(\mathrm{t}) \mathrm{dt}\right]^{2.5}\left(\mathrm{t}_{2}-\mathrm{t}_{1}\right)\right\}_{\max }
$$

where $t_{1}$ and $t_{2}$ correspond to the time values along the acceleration pulse that will maximise the HIC output. The time interval $t_{2}-t_{1}$ is previously specified according to safety regulations. At first, the NHTSA proposed a limit value of $36 \mathrm{~ms}$.

Parallelly, Stalnaker et al. (1971) [107] performed head impact experiments on monkeys whose results were employed to formulate the Mean Strain Criterion (MSC) which is a measure of the ratio between the cranial diameter after and before the impact. Complementing their study with PMHS tests, 
they suggested an extrapolation of the tolerable mean strain for human beings of 0.0061 .

In 1982, the ISO standard for Road Vehicles defined HIC as the safety parameter for evaluating frontal car crash collisions, defining a maximum value of 1500 , based on the experimental tests on brain injury on PMHS carried out by Got et al. (1978) [108] and Tarriere et al. (1982) [109]. However, this value was substantially higher than the one proposed by the Federal Motor Vehicle Safety Standard (FMVSS) 208 and therefore an Advisory Group was created to establish suitable limits to HIC based on biomechanical impact data. As a result, Prasad and Mertz (1985) [110] published a report in which some improvements for HIC were proposed using cadaveric tests from the existing literature. Their approach aimed to establish an adequate tolerance curve able to account both for brain injury and skull fracture prediction. From their experimental database they realised that in some cases brain injuries and skull fractures were sustained for HIC values between 1000 and 1500, which establishes $\mathrm{HIC}=1500$ as a non-conservative value for injury prediction. Additionally, Prasad and Mertz suggested the limitation of the HIC interval to $15 \mathrm{~ms}$, due to the unlikeliness of experimenting head injury for longer pulses with low acceleration levels. Cumulative probability curves based on normal distributions (following the trend of the study by Mertz and Weber (1982) [111]) were then proposed for brain injury and skull fracture risk as functions of the HIC level.

Some posterior works aimed to improve the accuracy of skull fracture prediction using HIC as a variable. For instance, Hertz (1993) [112] took experimental data on tests with PMHS and proposed a skull fracture probability curve which consisted on a lognormal distribution of the HIC, defined by Eq. 2.24 .

$$
p(\text { frac })=\mathrm{N}\left(\frac{\ln \mathrm{HIC}-\mu}{\sigma}\right)
$$

being $\mu=6.96352$ and $\sigma=0.84664$.

These cumulative probability functions were compared by Ruan and Prasad (2001) [113] in a study employing finite element numerical head models with different skull thicknesses. In their simulations, half-sine acceleration pulses were applied to the head models, and they considered that skull fracture occured when the cortical bone reached a von Mises stress value of $100 \mathrm{MPa}$. 
They represented their data together with the cranial fracture probability curves by Prasad and Mertz (1985) [110], Mertz (1996) [114] and Hertz (1993) [112]. The latter curve proved not to fit the data from the numerical simulations, while the one developed by Mertz was in good agreement with them, for all the cranial thicknesses considered.

Posteriorly, Vander Vorst et al. (2003) [115] suggested a new skull fracture predictor under frontal impact conditions based on data from three sources: experimental tests on PMHS from the literature, drop tests on Hybrid III headforms and numerical simulations with a FE head model. With this procedure, they aimed to relate the occurrence of skull fracture from the experimental values with the strain levels recorded at the cranial frontal bone in the simulations. Then, the fracture threshold in terms of bone strain is statistically correlated to the parameters measurable by the Anthropomorphic Test Devices (ATDs). The result of this study is the Skull Fracture Correlate (SFC), defined by Eq. 2.25 as the mean acceleration during the HIC interval.

$$
S F C=\frac{\Delta V_{\mathrm{HIC}}}{\Delta T_{\mathrm{HIC}}}=\frac{\int_{t_{1}}^{t_{2}} \mathrm{a}(\mathrm{t}) \mathrm{dt}}{\left(t_{2}-t_{1}\right)}
$$

They emphasized the usefulness of this parameter due to its good correlation with skull strains that cause frontal linear fractures, and because it is independent of the contact area and the impacting material. A value of SFC lower than $120 \mathrm{~g}$ is related to a skull fracture probability lower than $15 \%$. A later work by the same authors [116] for lateral skull fracture concludes that the same criterion developed for frontal bone in terms of SFC applies to the temporoparietal region.

Nonetheless, posterior research works suggested that rotational acceleration may be more relevant as an injury trigger, and pure traslational acceleration-based criteria like HIC and its derivates were considered unsuitable for predicting several types of head injuries.

\section{Understanding the importance of rotation}

In 1986, the first criterion combining both translational and rotational accelerations was proposed by Newman [117], and was named GAMBIT (generalized 
acceleration model for brain injury threshold). Basing on preceeding experimental tests on post-mortem human and animals, the parameters $n, m, S, a_{C}$ and $\alpha_{C}$ in Eq. 2.26 were adjusted to achieve the final form of the criterion (Eq. 2.27) which defines a straight line.

$$
\begin{gathered}
G(t)=\left[\left(\frac{a(t)}{a_{C}}\right)^{n}+\left(\frac{\alpha(t)}{\alpha_{C}}\right)^{m}\right]^{1 / S} \\
G=\frac{a_{m}}{250}+\frac{\alpha_{m}}{10000} \leq 1
\end{gathered}
$$

The parameters employed to ponderate the translational and rotational accelerations were $250 \mathrm{~g}$ and $10000 \mathrm{rads}^{-1}$, respectively, and were taken from other injury regulations. However, the final GAMBIT expression was not widely employed because it lacked an experimental validation. An improved combined criteria was posteriorly defined by the same autor [118], on the assumption that injury will occur at the maximum rate of change of kinetic energy of the head. This gives as a result a novel criterion, the Head Impact Power (HIP, Eq. 2.28):

$$
\begin{aligned}
\mathrm{HIP} & =4.50 \mathrm{a}_{\mathrm{x}} \int \mathrm{a}_{\mathrm{x}} \mathrm{dt}+4.50 \mathrm{a}_{\mathrm{y}} \int \mathrm{a}_{\mathrm{y}} \mathrm{dt}+4.50 \mathrm{a}_{\mathrm{z}} \int \mathrm{a}_{\mathrm{z}} \mathrm{dt}+ \\
& +0.016 \alpha_{\mathrm{x}} \int \alpha_{\mathrm{x}} \mathrm{dt}+0.024 \alpha_{\mathrm{y}} \int \alpha_{\mathrm{y}} \mathrm{dt}+0.022 \alpha_{\mathrm{z}} \int \alpha_{\mathrm{z}} \mathrm{dt}
\end{aligned}
$$

Departing from this hypothesis, injury risk curves were obtained from the reconstruction of accidental collisions of 24 professional football players by means of Hybrid III headforms. With this dataset, Newman et al. [119] compared HIP with other acceleration-based criteria in terms of statistical indexes. They obtained that the best correlation was obtained with HIP, and proposed a value of $12.8 \mathrm{~kW}$ for a $50 \%$ probability of mild concussion.

Rotational kinematics subsequent to an impact in the human head are meant to be the primary mechanism of diffuse brain injuries. While some studies take rotational accelerations as an injury predictor, other works suggest that the shear response sustained in the brain tissue may give a more specific quantification of the actual injury level. The first study presenting shear as a mechanism of brain injury was the one by Holbourn (1943) [120], 
employing a synthetic brain model (gelatin) protected by a surrogate skull (wax) upon which rotational loads were applied. In his work brain injuries were considered to be caused by two mechanisms: skull deformation and its fracture, and motion-related injuries independent of skull response. In this last mechanism, it was stated that translational accelerations had little contribution to the shear strains in the brain, being most of them caused by rotational forces. Another relevant finding comes from this work: rotational accelerations may be proportional to shear strains for long duration loadings, while for short duration events it is rotational velocity which correlates to injury. Posteriorly, the works by Strich in 1956 [121] and 1961 [122] supported the hypothesis of the injury potential of shear strain by histologically examining white matter tissues of 20 human patients presenting diffuse brain injuries. She found morphological alterations both in the neuronal axons and in blood vessels which were result of rotational forces.

Based on these pioneer studies Peerless and Rewcastle (1967) [123] continued the characterisation of brain injuries. In their work, clinical cases of 37 patients with different symptoms but all of them resulting in death were examined. It was found that all cases presented damage in the splenium of the corpus callosum. Furthermore, axonal dysruptions and small haemorrhages, located mainly in the diencephalon region, were also present in all cases. Observation allowed to detect bulbs and ruptures in the axonal bodies, which were attributed to the presence of shear forces. The hypothesis of DAI being caused by angular acceleration was further confirmed by Ommaya and Gennarelli (1975) [124], who performed pure inertial loading tests on squirrel monkeys. In this case, the stimulus applied was either translational or rotational so the injury outcomes from each acceleration type could be clearly separated. The resulting lesions from the rotated specimens presented all a similar trend, achieving concussion, subdural hematoma and subarachnoid haemorrhages in all cases (loaded with accelerations ranging from 348 to 1025 g). On the contrary, pure translated specimens revealed less injurious patterns after examination. None of these cases achieved concussion and only focal lesions were found in some cases, even if the peak accelerations applied were higher than for the rotations (from 665 to 1230g). Another of their findings was that the most severe rotational injuries coincided with the cases in which the upper brainstem is damaged, postulating that DAI can occur without involving lesions to the brainstem, and that these last only happen when the outer regions of the brain have been extensively injured. 
Additional findings of the rotational contribution to DAI were achieved by Gennarelli et al. (1982) [125] with their experimental tests on primates. Angular acceleration pulses of approximately $200 \mathrm{krads}^{-2}$ were imparted to the head of the 45 specimens, at various axes of rotation. They obtained different levels of injury, which could be quantified by the duration of coma and the post-traumatic abnormalities, which were dependent on the loading direction. The subjects submitted to coronal plane accelerations (lateral rotation of the head) presented the most severe manifestations of DAI, which was microscopically observable by the presence of disperse retraction balls (from the damaged axons) along the white matter. Departing from this last fact, Margulies and Thibault (1992) [126] performed lateral rotation tests (coronal plane) on anesthetized baboons in an extent sufficient to cause coma. Applying scaling techniques and assuming that the critical brain strain to achieve DAI is located at the deep white matter, tolerance curves for humans were built in the shape of peak angular acceleration and peak angular velocity. They suggested that this curve could be extended to different brain mass values. Nonetheless, the critical strain value obtained by the authors was set under certain hypotheses and does not necessarily correspond to the actual stretching of the white matter tissue at which physical damage occurs. The change of this critical value involves different resultant tolerance curves.

These works gave a better understanding of the biomechanics of DAI, and enhanced the characterisation of this rotational-based injury mechanism with numerical human head models. In this context, Bandak and Eppinger (1994) [127] performed explicit FE simulations on a head model under different kinematic boundary conditions, including pure rotations, pure translations or a combination of both. Based on the assumption that the severity of axonal functional injury can be regarded as a cumulative phenomenom, they proposed a new injury metric to assess DAI under impact conditions: the Cumulative Strain Damage Measure (CSDM). This metric is defined as the brain volume ratio that during an impact event has experienced strain levels greater than a certain threshold value. However, experimental data to establish axonal strain tolerance values and CSDM risk curves were not provided and therefore the measure was still not directly appliable in the prediction of DAI through numerical modeling.

Other injury criteria were also drawn by means of finite element models. This is the case of Takhounts et al. (2003) [128], who built a numerical human head model able to predict different injury modes. Apart from employing 
the previously mentioned CSDM for DAI prediction, two additional criteria were proposed for the evaluation of contusions and subdural hematoma, respectively to be applied to FE models. The first one, named the Dilatation Damage Measure (DDM), represents the percentage of brain tissue that during an impact event has withstood pressure levels below $-100 \mathrm{kPa}$, which is approximately the water cavitation pressure. The second injury metric established in [128] is the so-called Relative Motion Damage Measure (RMDM) and employs the relative displacement between pairs of nodes in the brain and the skull to compute both the strain and strain rate of 'fictitious' bridging veins joining both structures.

\section{Addressing axonal disruption}

Posteriorly, using data of football players sustaining different head injury levels, Zang et al. (2004) [129] aimed to develop a predictor for mild TBI. They simulated the impact scenarios of the football players using both an experimental set-up with helmeted Hybrid III dummies and a modification of the head numerical model developed by Zhang et al. [130]. They assessed different injury criteria like the HIC, GSI, peak translational and rotational accelerations, intracranial pressure and shear stress. A regression analysis revealed that the best injury correlator for mild lessions was the shear stess at the upper brainstem (being this region the one with the highest concentration of shear stresses). In this work a shear stress value of $7.8 \mathrm{kPa}$ was proposed to correspond to a $50 \%$ probability of sustaining mild traumatic brain injuries.

On the other hand, obtaining experimental in vivo tissue-level thresholds for the different brain lesions became an important issue due to its likely application to injury predictive finite element models. Shreiber et al. (1997) [131] performed a pioneering work in determining tolerance strain values of the brain tissue for focal (contusive) lesions. To this purpose, they conducted experimental tests on rats, upon which a specific area of the intracranial cavity was depressurized. Damage was quantified through fluorescence of certain particles transpassing the blood vessel barriers. Posterior FE reconstruction of the experiments led to consider maximum principal logarithmic strain as the most robust predictor of cortical injury, setting a threshold value of 0.188 between non-injured and injured cases. Despite accounting for several simplifying assumptions and employing animal specimens, this tolerance value gave an order of magnitude that could be close for the human in contusive lesions. 
The first study to provide quantitative in vivo data for the disruption thresholds of the white matter axonal tissue was performed by Bain and Meaney in 2000 [132]. 70 experimental tests were performed on the optical nerve of guinea pigs by imposing certain displacement values on the ocular balls that implied a specific Lagrangian strain in the axonal body of the nerve. After the tests, the specimens were examined and morphological injury was determined through the use of biomarkers able to detect axonal swelling and the presence of retraction balls, both consequences of DAI. To determine the functional aftermaths of the tests, the electrical potentials carried by the optic nerve were monitored, indicating abnormal functioning when the signal peaks appear delayed from the reference taken before testing. Morphological examination revealed 33 injured cases whose clinical severity was proportional to the prescribed eyeball displacement. The threshold strains for this kind of injury were based on a logistic regression, setting values of $0.14,0.34$ and 0.21 for $5 \%, 90 \%$ and $25 \%$ of injury probability, respectively. Analogously, the strain thresholds for functional impairment in the optic nerve were 0.13 (conservative), 0.28 (liberal) and 0.181 (optimal). In both injury types it was demonstrated that optical nerve strain was a good injury correlator.

Another approach for the stretching tolerance levels of the brain tissue was given later by Morrison et al. (2003) [133] employing an in vitro testing environment. In this case, the test specimen consisted of sliced cultures from the hippocampal region of the rat brain, due to the implication of this structure (the hippocampus) in the loss of certain basic functions under traumatic injury. Biaxial loading of the specimens was performed controlling the maximal Lagrangian strain values and the strain rates. Cell death in the hippocampal region was identified by means of biomarkers which permit to visualise injury inferred to axons. Their results did not give accurate quantitative tolerance data in terms of strain but provided a procedure of recreating TBI in vitro. Some numerical conclusions were also drawn, for example the sensitivity of brain tissue to strain rate, given an increased risk of TBI when increasing this parameter. Furthermore, despite the clearest manifestations of TBI were obtained for large strain values (severe injury conditions) the authors suggested that a reasonable tolerance strain value could be found around 0.2 in terms of lagrangian strain, which falls in the range of the proposed by Bain and Meaney [132]. 
Through finite element reconstruction of 109 accidental impact cases, Sahoo et al. (2016) [134] studied the suitability of different brain injury criteria for estimating DAI. With their Diffusion Tensor Imaging (DTI)-based anisotropic brain model, they proposed the maximum axonal strain as the best injury correlator, setting a threshold of $14.65 \%$ axonal elongation for a $50 \%$ probability of DAI. Their study also validated the criteria proposed by Takhounts et al. [128] based on CSDM, who established that with a threshold strain of $15 \%$ and a CSDM value of the $55 \%$, there is a $50 \%$ probability of DAI. The main difference between both criteria is whether considering the directions of the axonal lattice (direct axonal strain) or conceiving the brain as an homogeneous tissue and therefore employ a cumulative measure (CSDM).

\section{Focal injuries: contusions and haemorrhages}

Monea et al. (2014) [135] employed experimental data from previous publications to establish mechanically-based injury metrics for various head lesions. Their work led to the conclusion that both brain contusion and acute subdural hematoma (ASDH) could be suitably predicted by a measure proportional to the square of the product of the angular acceleration times the pulse duration $(\alpha \Delta t)^{2}$. Furthermore, occurrence of ASDH was determined to be linked to stretching in the bridging veins which communicate the cerebral cortex with the superior sagittal sinus, when their deformation exceeded the $25 \%$. This threshold value was obtained by Delye et al. (2006) [136], and was significantly lower than previous ultimate strains reported in the literature which were established around a $50 \%$ value. Human tolerance to ASDH was also experimentally addresed by Depreitere et al. (2006) [137] performing impact tests on ten unembalmed human cadavers. Rupture of bridging veins, which took place in six of the cases, was determined by means of fluoroscopy. They reported tolerance values in terms of peak rotational accelerations for a given pulse length, setting a threshold value of $10 \mathrm{krads}^{-2}$ for pulses shorter than $10 \mathrm{~ms}$.

Mechanical and failure properties of bridging veins have been investigated in the literature due to their potential link in the mechanisms of subdural hematoma. Nonetheless, differences in their morphology and arrangement along the intracranial space make it difficult to establish robust tolerance criteria based on experimental tests, which normally consist of uniaxial loading of isolated veins from postmortem specimens. Monson et al. performed dif- 
ferent studies on the mechanical properties of the intracranial vasculature. In their work from 2003 [138], in which different vessels obtained from surgery were tested, they obtained that under axial loading condition the brain arteries presented a greater stiffness and strength than veins. Employing the same loading conditions but with specimens harvested from cadavers, a posterior study in 2005 [139] examined a wider sample of intracranial blood vessels of different typologies, including arteries, bridging veins and smaller cortical vessels. The mechanical response of arteries revealed a similar trend if compared to the surgically-obtained analog vessels, the latter showing lower stiffness values and strengths, but withstanding more stretching until failure. Cortical veins showed the lowest stiffnesses (average modulus of $3.46 \mathrm{MPa}$ ) followed by bridging veins $(\approx 6.43 \mathrm{MPa})$ and the elongation to failure differed from 1.72 to 1.5 , respectively. Size of the vessels had a prominent effect on their behaviour, obtaining significantly greater stiffness values for smaller arteries than large ones, whereas in the case of veins the trend was inverted and larger veins (bridging) were much stiffer than smaller ones.

Monea et al. (2014) [140] characterised the mechanical response of bridging veins under tensile stresses up to failure at different loading rates. In their examination of the geometry of 113 specimens, a general morphological pattern was observed: a larger cross section was found in the interface between the vein and the superior sagittal sinus, followed by a neck portion lying on the subdural space and finally a progressive narrowing along the segment inmersed in the arachnoidal region. Mechanical failure was in most of the cases initialised at the mid segment of the vein body, fact that suggests a plausible blood leakage in the subdural space when the vein wall is ruptured. For loading rates up to $60 \mathrm{~s}^{-1}$ the reported average ultimate strain was approximately a $23.8 \%$, while greater rates (up to $200 \mathrm{~s}^{-1}$ ) involved a greater value $(33.5 \%$ ).

Apart from hematomas, other focal injuries such as contusions or haemorrhages in the brain are consequence of pressure gradients, caused by intracranial pressure waves within the skull boundaries. The work by Ward et al. (1980) [141] was one of the first to experimentally demonstrate a correlation between coup intracranial pressures and brain injury. As a result, they proposed a new criterion, the brain pressure tolerance (BPT), which presented the same format of the WSTC but was more restrictive in terms of acceptable head accelerations for the short duration pulses (0 to $3 \mathrm{~ms}$ ). Their proposed coup pressure threshold for severe injury, $234 \mathrm{kPa}$, has been widely used in numerical prediction of head trauma. 
Another risk phenomenom for focal injuries, even though still not fully clarified and quantified, is given when the brain withstands negative pressures below the water vapour pressure as cavitation processes may be triggered $[142,143]$. The first study to address this issue was performed by Gross in 1958 [144] performing experimental impact tests on a simplified head model consisting of an ellipsoidal glass vessel filled with fluid. Even though in this study cavitation bubbles were observed, it remained unclear whether the effect in a real human head would be similar. To bring some light to this question, in 1980 Lubock and Goldsmith [145] conducted a similar study employing fluidfilled PMHS skulls and obtained cavitation bubbles at the countrecoup region. Nonetheless, a second batch of tests were performed with a gelatin filling the intracranial space and no trace of cavitation was found. From these study two hypothesis were drawn: the brain itself does not appear to be susceptible to cavitation phenomena but it may be feasible to affect the intracranial fluids, including the CSF and blood vessels on the brain surface. This last finding suggests the development of focal head injuries from cavitation despite not being backed up by any clinical evidence [146, 147]. 
Chapter 2. Head biomechanics

\begin{tabular}{|c|c|c|c|c|}
\hline Injury type & Criterion & Parameter & Threshold & Ref. \\
\hline \multirow{9}{*}{ Skull fracture } & GSI & $a_{t}$ & 1000 & {$[103]$} \\
\hline & $\mathrm{HIC}$ & $a_{t}$ & 1500 & {$[108]$} \\
\hline & $p($ frac $)$ & $a_{t}$ & measured as probability & {$[112]$} \\
\hline & $\mathrm{SFC}$ & $a_{t}$ & $120 \mathrm{~g}$ for $15 \%$ risk & {$[115]$} \\
\hline & $\mathrm{MSC}$ & skull strain & 0.0061 & {$[107]$} \\
\hline & MPS & $\begin{array}{l}\sigma_{u t} \quad \text { (compact } \\
\text { bone) }\end{array}$ & $90 \mathrm{MPa}$ & {$[148]$} \\
\hline & MPS & $\begin{array}{l}\sigma_{u c} \quad(\text { compact } \\
\text { bone) }\end{array}$ & $-132 \mathrm{MPa}$ & {$[148]$} \\
\hline & MPS & $\sigma_{u t}($ diplöe $)$ & $34.8 \mathrm{MPa}$ & {$[148]$} \\
\hline & MPS & $\sigma_{u c}($ diplöe $)$ & $-24.8 \mathrm{MPa}$ & {$[148]$} \\
\hline \multirow{6}{*}{ DAI } & HIP & $a_{t}$ and $\alpha$ & $\begin{array}{l}12.8 \mathrm{~kW} \text { for } 50 \% \text { risk of } \\
\text { mild concussion }\end{array}$ & {$[117]$} \\
\hline & - & peak $\alpha$ & $\begin{array}{l}1.8 \mathrm{krads}^{-2} \text { for pulses } \\
\text { shorter than } 20 \mathrm{~ms} \text { [149] }\end{array}$ & \\
\hline & CSDM & brain strain & $\begin{array}{l}55 \% \text { CSDM for } 50 \% \\
\text { DAI risk }\end{array}$ & {$[128]$} \\
\hline & - & $\begin{array}{l}\text { shear stress at } \\
\text { brainstem }\end{array}$ & $\begin{array}{l}7.8 \mathrm{kPa} \text { for } 50 \% \mathrm{mTBI} \\
\text { risk }\end{array}$ & [129] \\
\hline & - & $\begin{array}{l}\text { lagrangian } \\
\text { axonal strain }\end{array}$ & 0.2 & {$[133]$} \\
\hline & - & axonal strain & 0.1465 for $50 \%$ DAI risk & {$[134]$} \\
\hline \multirow[t]{3}{*}{ SDH } & - & $\begin{array}{l}\text { strain in bridg- } \\
\text { ing veins }\end{array}$ & 0.25 for acute SDH & {$[136]$} \\
\hline & - & $\begin{array}{l}\text { strain in bridg- } \\
\text { ing veins }\end{array}$ & 0.5 & {$[140]$} \\
\hline & - & peak $\alpha$ & $\begin{array}{l}10 \mathrm{krads}^{-2} \text { for pulses } \\
\text { shorter than } 10 \mathrm{~ms}\end{array}$ & {$[137]$} \\
\hline \multirow{2}{*}{ Contusions } & $\mathrm{DDM}$ & negative ICP & $7.2 \%$ for $50 \%$ risk & {$[128]$} \\
\hline & $\mathrm{BPT}$ & peak ICP & $\begin{array}{l}234 \mathrm{kPa} \text { for severe in- } \\
\text { jury }\end{array}$ & {$[141]$} \\
\hline
\end{tabular}

Table 2.5: Summary of some published head injury criteria. 


\title{
Chapter 3
}

\section{Explicit Finite Element Analysis}

\author{
Understanding is a kind of \\ ecstasy.
}

- Carl Sagan

Given that the purpose of this Thesis is the study of the impact behaviour of living human tissues and protective materials through FEA, it is convenient to present certain features that characterise the preferred computation method for non-linear dynamics: the explicit FEM.

\subsection{Equations of transient dynamics}

Before presenting the actual fundamentals of the explicit FEM, it is necessary to establish some background about the equations governing transient dynamics. Considering a system of $n$ particles, the expression of d'Alembert's principle in the absence of non-conservative forces only depends on the potential (V) and kinetic energy of the particles [150]:

$$
\sum_{i=1}^{n}\left(m_{i} \frac{\mathrm{d}^{2} \mathbf{r}_{i}}{\mathrm{~d} t^{2}}+\frac{\partial V}{\partial \mathbf{r}_{i}}\right) \cdot \delta \mathbf{r}_{i}=0
$$


being $\mathbf{r}_{i}$ the position vector of each particle and $\delta \mathbf{r}_{1}$ its virtual displacement. Decomposing Eq. 3.1, the variation of each term in terms of $\mathbf{r}$ can be expressed as follows:

$$
\begin{aligned}
& \delta V=\sum_{i=1}^{n}\left(\frac{\partial V}{\partial \mathbf{r}_{i}}\right) \cdot \delta \mathbf{r}_{i} \\
& \frac{\mathrm{d}}{\mathrm{d} t}\left[\sum_{i=1}^{n}\left(m_{i} \frac{\mathrm{d} \mathbf{r}_{i}}{\mathrm{~d} t}\right) \cdot \delta \mathbf{r}_{i}\right]=\sum_{i=1}^{n}\left(m_{i} \frac{\mathrm{d}^{2} \mathbf{r}_{i}}{\mathrm{~d} t^{2}}\right) \cdot \delta \mathbf{r}_{i}+\delta \sum_{i=1}^{n} \frac{m_{i}}{2} \frac{\mathrm{d} \mathbf{r}_{\mathrm{i}}}{\mathrm{d} t} \cdot \frac{\mathrm{d} \mathbf{r}_{\mathrm{i}}}{\mathrm{d} t} \\
& =\sum_{i=1}^{n}\left(m_{i} \frac{\mathrm{d}^{2} \mathbf{r}_{i}}{\mathrm{~d} t^{2}}\right) \cdot \delta \mathbf{r}_{i}+\delta T
\end{aligned}
$$

Having introduced both energy terms $V$ and $T$ in Eq. 3.1, it can be rewritten as a function of the Lagrangian potential $L=T-V$ :

$$
\delta L=\frac{\mathrm{d}}{\mathrm{d} t}\left[\sum_{i=1}^{n}\left(m_{i} \frac{\mathrm{d} \mathbf{r}_{i}}{\mathrm{~d} t}\right) \cdot \delta \mathbf{r}_{i}\right]
$$

Performing time integration on both sides of Eq. 3.4 will lead to the basis of the Hamilton's Principle of classic mechanics for a conservative system:

$$
\delta \mathcal{L}=\int_{t_{1}}^{t_{2}} \delta L \mathrm{~d} t=\left[\sum_{i=1}^{n}\left(m_{i} \frac{\mathrm{d} \mathbf{r}_{i}}{\mathrm{~d} t}\right) \cdot \delta \mathbf{r}_{i}\right]_{t_{1}}^{t_{2}}
$$

According to this principle, any displacement of the particles of the system that does not belong to the true path at times $t_{1}$ and $t_{2}$ leads to a configuration which is not possible to achieve. Therefore, the virtual displacements $\delta \mathbf{r}_{\mathrm{i}}$ must be zero. As a result:

$$
\delta \mathcal{L}=\int_{t_{1}}^{t_{2}}(\delta T-\delta V) \mathrm{d} t=0
$$

Denoting by $u_{i}$ the displacement of the system at a certain domain $\Gamma_{u}$, the kinetic energy term can be rewritten as 


$$
\delta T=\int_{\Omega} \rho \dot{u}_{i} \delta \dot{u}_{i} \mathrm{~d} \Omega
$$

where $\Omega$ represents the whole domain of the system upon which body forces $f_{i}$ are acting. Furthermore, considering the presence of boundary forces $g_{i}$ on a region $\Gamma_{s}$, the potential energy term of Eq. 3.5 is expressed as follows:

$$
\delta V=\int_{\Omega} \sigma_{i j} \delta \varepsilon_{i j} \mathrm{~d} \Omega-\int_{\Omega} f_{i} \delta u_{i} \mathrm{~d} \Omega-\int_{\Gamma_{s}} g_{i} \delta u_{i} \mathrm{~d} \Gamma
$$

The first term on the right of Eq. 3.8 can be integrated by parts. If assuming small displacements, it is accomplished that $\dot{\varepsilon}_{i j}=\frac{\left(\dot{u}_{i, j}+\dot{u}_{j, i}\right)}{2}$, and subsequently

$$
\int_{\Omega} \sigma_{i j} \delta \varepsilon_{i j} \mathrm{~d} \Omega=-\int_{\Omega} \sigma_{i j, j} \delta u_{i} \mathrm{~d} \Omega+\int_{\Gamma_{s}} \sigma_{i j} n_{j} \delta u_{i} \mathrm{~d} \Gamma
$$

The expression of Hamilton's principle can then be rearranged as

$$
\begin{aligned}
\int_{t_{1}}^{t_{2}}\left(\int_{\Omega}-\rho \ddot{u}_{i} \delta u_{i} \mathrm{~d} \Omega+\int_{\Omega} \sigma_{i j, j} \delta u_{i} \mathrm{~d} \Omega\right. & \\
& \left.\quad-\int_{\Gamma_{s}} \sigma_{i j} n_{j} \delta u_{i} \mathrm{~d} \Gamma+\int_{\Omega} f_{i} \delta u_{i} \mathrm{~d} \Omega+\int_{\Gamma_{s}} g_{i} \delta u_{i} \mathrm{~d} \Gamma\right) \mathrm{d} t=0
\end{aligned}
$$

An analogous method to assess the equations for transient dynamics of a system is by means of the Galerkin method, that consists of the direct integration of the motion and force equations and the inclusion of certain test functions $v_{i}$ that must fulfil the boundary conditions of the problem. The resulting variational equation is mainly equivalent to Eq. 3.10:

$$
\int_{\Omega}\left(\rho \ddot{u}_{i} v_{i}+\sigma_{i j} v_{i, j}\right) \mathrm{d} \Omega=\int_{\Omega} f_{i} v_{i} \mathrm{~d} \Omega+\int_{\Gamma_{s}} g_{i} v_{i} \mathrm{~d} \Gamma
$$




\subsubsection{Discretisation in space}

The conversion of Eq. 3.11 into a valid equation for finite element analysis in the space domain is straightforward by making the test functions $v_{i}$ nodaldependent, this means, introducing the element shape functions $\Phi_{N}$ for nodal interpolation: $v_{i}=v_{i}^{N} \Phi_{N}$.

$$
\int_{\Omega}\left(\rho \Phi_{M} \Phi_{N} \ddot{u}_{i}^{N}+\sigma_{i j} \Phi_{M, j}\right) v_{i}^{M} \mathrm{~d} \Omega=\int_{\Omega} f_{i} \Phi_{M} v_{i}^{M} \mathrm{~d} \Omega+\int_{\Gamma_{s}} g_{i} \Phi_{M} v_{i}^{M} \mathrm{~d} \Gamma
$$

It is important to point out that the functions $\Phi_{N}$. are only node-dependent and not time dependent. Therefore, the only terms in Eq. 3.12 that possess time dependency are the nodal acceleration $\ddot{u}_{i}^{N}$ and the test functions $v_{i}^{M}$. As these latter are arbitrary functions, they can be simplified from Eq. 3.12, which can therefore be rewritten in the form of the second's Newton law [151]:

$$
\sum_{N} \underbrace{\int_{\Omega}\left(\rho \Phi_{M} \Phi_{N}\right) \mathrm{d} \Omega}_{M_{M N}} \underbrace{\ddot{u}_{i}^{N}(t)}_{A_{i}^{N}}=\underbrace{-\int_{\Omega}\left(\sigma_{i j} \Phi_{M, j}\right) \mathrm{d} \Omega+\int_{\Omega} f_{i} \Phi_{M} \mathrm{~d} \Omega+\int_{\Gamma_{s}} g_{i} \Phi_{M} \mathrm{~d} \Gamma}_{F_{i}^{M}}
$$

Compacting Eq. 3.13 in matrix notation, we set the basis for the calculation of dynamic problems using the finite element method:

$$
\mathbf{F}=\mathbf{M} A
$$

\subsection{Time dependency: implicit and explicit FEA}

The system of second-order ODEs defined by Eq.3.14 can be solved using different approaches which are generally classified as implicit or explicit methods. Implicit methods require to solve the motion equations at the current time step to calculate the current displacements. On the other hand, explicit 
methods employ step-by-step approaches and do not require to solve the system of equations at every time step, as displacements and its derivatives can be directly obtained from the previous step. This feature makes explicit methods more efficient when dealing with large numbers of degrees of freedom and highly nonlinear problems.

\subsubsection{Implicit schemes}

The most widely employed algorithms for implicit calculation are based on Newmark's methods for integration, whose main equations for velocity and displacement computation are

$$
\begin{gathered}
\dot{\mathbf{u}}^{(i+1)}=\dot{\mathbf{u}}^{(i)}+\Delta t\left((1-\gamma) \ddot{\mathbf{u}}^{(i)}+\gamma \ddot{\mathbf{u}}^{(i+1)}\right) \\
\mathbf{u}^{(i+1)}=\mathbf{u}^{(i)}+\Delta t \dot{\mathbf{u}}^{(i)}+\Delta t^{2}\left(\left(\frac{1}{2}-\beta\right) \ddot{\mathbf{u}}^{(i)}+\beta \ddot{\mathbf{u}}^{(i+1)}\right)
\end{gathered}
$$

and

$$
\beta=\frac{1}{4}\left(1-\alpha^{2}\right), \quad \gamma=\frac{1}{2}-\alpha, \quad-\frac{1}{2} \leq \alpha \leq 0
$$

The law of variation of the acceleration at every time step is determined by the constants $\beta$ and $\gamma$. The parameter $\alpha$ is often suitable in implicit calculation of dynamic problems as introduces certain damping and removes the high frequency noises [152]. For instance, in Abaqus/Standard, the default value is $\alpha=-0.05$. When $\alpha=0$, there is no damping and $\beta=\frac{1}{4}$ and $\gamma=\frac{1}{2}$ this special case is known as the average acceleration method.

\subsection{The explicit scheme}

Even though there exist other integration schemes, the explicit finite element method is normally based on the integration of the equations of motion employing the central difference method, and this will be the procedure explained in this chapter as it is the one implemented in Abaqus/Explicit. The equations of motion with the central difference method are the following: 


$$
\begin{gathered}
\dot{\mathbf{u}}^{\left(i+\frac{1}{2}\right)}=\dot{\mathbf{u}}^{\left(i-\frac{1}{2}\right)}+\frac{\Delta t^{(i+1)}+\Delta t^{(i)}}{2} \ddot{\mathbf{u}}^{(i)} \\
\mathbf{u}^{(i+1)}=\mathbf{u}^{(i)}+\Delta t^{(i+1)} \dot{\mathbf{u}}^{\left(i+\frac{1}{2}\right)}
\end{gathered}
$$

where $\dot{\mathbf{u}}$ and $\ddot{\mathbf{u}}$ represent velocity and acceleration, respectively, and $i$ is the increment number. It is relevant to note that in Eqs. 3.18 and 3.19 a variable time increment $\Delta t$ is assumed. As explained by Belytschko et al. [153], if a system is highly nonlinear, a varying time increment should be considered.

The explicit scheme using the central difference method can be summarised as follows (taken from Belytschko et al. [153]):

1. Set the initial conditions

$$
\mathbf{u}^{0}=\mathbf{u}(0), \quad \dot{\mathbf{u}}^{\frac{1}{2}}=\dot{\mathbf{u}}(0), \quad \mathbf{T}^{0}=\mathbf{T}(0), \quad i=0
$$

2. Displacements updating

$$
\mathbf{u}^{i+1}=\mathbf{u}^{i}+\Delta t^{i} \dot{\mathbf{u}}^{1+\frac{1}{2}}
$$

3. Computing the internal force matrix

(a)

$$
\mathbf{V}_{i j}=\mathbf{B}_{i j} \dot{\mathbf{u}}^{(e) i+\frac{1}{2}}, \quad \mathbf{D}_{i j}^{i+\frac{1}{2}}=\frac{1}{2}\left(\mathbf{V}_{i j}+\mathbf{V}_{j i}\right), \quad \mathbf{W}_{i j}^{i+\frac{1}{2}}=\frac{1}{2}\left(\mathbf{V}_{i j}+\mathbf{V}_{j i}\right)
$$

(b) Insert the material's constitutive law (in this case, based on a weak formulation)

$$
\begin{gathered}
\mathbf{T}^{\nabla i+\frac{1}{2}}=\mathbf{C}\left(\mathbf{T}^{i}, \mathbf{E}^{i}, \mathbf{D}^{i+\frac{1}{2}}\right) \mathbf{D}^{i+\frac{1}{2}} \\
\dot{\mathbf{T}}^{i+\frac{1}{2}}=\mathbf{T}^{\nabla i+\frac{1}{2}}+\mathbf{W}^{i+\frac{1}{2}} \mathbf{T}^{i}+\mathbf{T}^{i}\left(\mathbf{W}^{T}\right)^{i+\frac{1}{2}}
\end{gathered}
$$

(c) Update the stress tensor for each element

$$
\mathbf{T}^{i+1}=\mathbf{T}^{i}+\Delta t^{i} \dot{\mathbf{T}}^{i+\frac{1}{2}}
$$


(d) Compute $\omega_{\max }^{(e)}$ to obtain $\Delta t^{i+1}$

(e) Compute internal forces at the element level

$$
\mathbf{f}_{(e) i n t}^{i+1}=\int \mathbf{B}^{T} \mathbf{T} \mathrm{d} \Omega
$$

(f) Build the total internal forces vector for the system $\mathbf{f}_{(e) \text { int }}^{i+1} \rightarrow \mathbf{f}_{\text {int }}^{i+1}$

4. Compute external nodal forces $\mathbf{f}_{e x t}^{i+1}$

5. Update accelerations

$$
\ddot{\mathbf{u}}^{i+1}=\mathbf{M}^{-1}\left(\mathbf{f}_{\text {ext }}^{i+1}+\mathbf{f}_{i n t}^{i+1}\right)
$$

6. Convert the predicted accelerations at $i+1$ to the current $i$ and apply Eq. 3.18

7. State the initial conditions for the next iteration

$$
\dot{\mathbf{u}}^{i+\frac{1}{2}}=\mathbf{u}^{i-\frac{1}{2}}+\Delta t^{i+\frac{1}{2}} \ddot{\mathbf{u}}^{i}
$$

8. Implement results to step 2

\subsection{Conditional stability}

The explicit method, despite providing the time-saving feature of employing diagonal mass matrices, has the main drawback that is conditionally stable and therefore there exists a stability limit for the time increment employed for the calculation upon which the solution diverges. This time limit can be expressed in terms of the maximum eigenvalue of the system $\left(\omega_{\max }\right)$ as given by Courant's condition:

$$
\Delta t \leq \frac{2}{\omega_{\max }}
$$

and would be described as the lowest transition time required for a dilatation wave to go through any element of the mesh. This magnitude is proportional 
to the ratio of the element length and the wave propagation speed $c_{d}$ given by its material law through Lamé elastic constants $\hat{\lambda}$ and $\hat{\mu}$ :

$$
\begin{aligned}
& \Delta t=\min \left(\frac{\mathrm{L}_{\mathrm{e}}}{\mathrm{c}_{\mathrm{d}}}\right) \\
& \mathrm{c}_{\mathrm{d}}=\sqrt{\frac{(\hat{\lambda}+2 \hat{\mu})}{\rho}}
\end{aligned}
$$

The stability problem can be easily understood by means of an example extracted from $\mathrm{Wu}$ and $\mathrm{Gu}$ [151] of an elastic rod with its both ends sumbitted to a constant velocity $v_{0}$, as illustrated in Fig. 3.1.

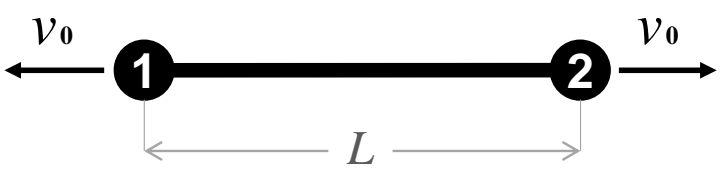

Figure 3.1: Rod element with an initial velocity.

Following the steps of the explicit scheme presented previously, it is immediate to achieve the displacement value of the two nodes of the truss at time $t=2$ :

- Displacement update

$$
\begin{aligned}
& u_{1}^{1}=u_{1}^{0}+\dot{u}_{1}^{0.5} \Delta t=-v_{0} \Delta t \\
& u_{2}^{1}=v_{0} \Delta t
\end{aligned}
$$

where $u_{1}^{0}=0$ and $\dot{u}_{1}^{0.5}=-v_{0}$.

- Force calculation

$$
\begin{aligned}
& \varepsilon^{1}=\frac{\left(u_{2}^{1}-u_{1}^{1}\right)}{L}=\frac{2 v_{0} \Delta t}{L} \\
& \sigma^{1}=E \frac{2 v_{0} \Delta t}{L} \\
& f^{1}=\mathrm{A} E \frac{2 v_{0} \Delta t}{L}
\end{aligned}
$$


- Compute accelerations

$$
\begin{aligned}
& \ddot{u}_{1}^{1}=\frac{f^{1}}{m} \\
& \ddot{u}_{2}^{1}=-\frac{f^{1}}{m}
\end{aligned}
$$

- Calculate the new velocities

$$
\begin{aligned}
& \dot{u}_{1}^{1.5}=\dot{u}_{1}^{0.5}+\ddot{u}_{1}^{1} \Delta t=-v_{0}+\mathrm{A} E \frac{2 v_{0} \Delta t^{2}}{m L} \\
& \dot{u}_{2}^{1.5}=v_{0}\left(1-\mathrm{A} E \frac{2 \Delta t^{2}}{m L}\right)=v_{0}\left(1-\frac{4 E \Delta t^{2}}{\rho L^{2}}\right)
\end{aligned}
$$

- Obtain displacements at the next time step

$$
\begin{aligned}
& u_{1}^{2}=u_{1}^{1}+\dot{u}_{1}^{1.5} \Delta t=-v_{0} \Delta t\left(2-\frac{4 E \Delta t^{2}}{\rho L^{2}}\right)=-v_{0} \Delta t\left(2-\omega^{2} \Delta t^{2}\right) \\
& u_{2}^{2}=v_{0} \Delta t\left(2-\omega^{2} \Delta t^{2}\right)
\end{aligned}
$$

$$
\text { where } \omega^{2}=\frac{4}{\frac{\rho L^{2}}{E}} \text { and } \omega=\frac{2}{\sqrt{\frac{\rho L^{2}}{E}}} \text {. }
$$

Giving arbitrary values to the different constants of the problem, i.e. $E=$ 200, $\rho=8 \cdot 10^{-6}, L=20, A=10$, the resulting natural frequency of the system is 500, and therefore the critical step given by Courant's principle is $\Delta t=4 \cdot 10^{-3}$. Fig. 3.2 displays the solutions to the rod problem setting a time step equal to the critical step $(\Delta t=0.004)$ and a value 10 times lower $(\Delta t=0.0004)$. The first case leads to a divergent solution to the displacement of each node, as the factor multiplying $v_{0}$ increases at each time increment. On the other hand, the lower time step leads to a convergent displacement which follows the shape of the analytical solution. 

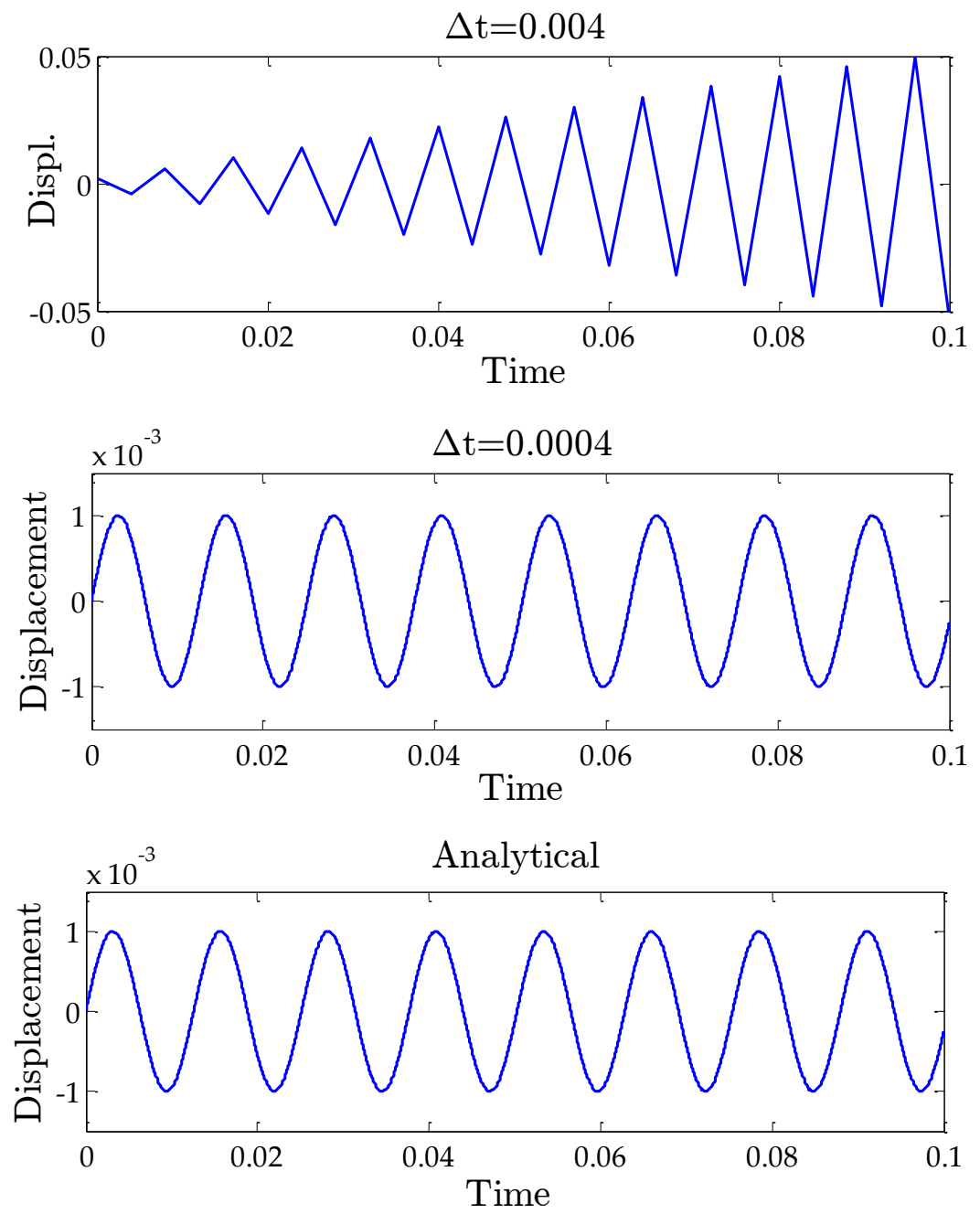

Figure 3.2: Explicit solution of the rod problem using different time step values and analytical solution of the problem. 


\subsection{Viscous damping}

One feature that explicit FEA usually introduces is a certain damping value in order to improve the stability of the calculation. This fact becomes especially beneficial when dealing with high-rate dynamic problems in which high frequency oscillations may arise [154]. Nonetheless, the introduction of this small damping has the drawback of diminishing the stable time increment [155]:

$$
\Delta t \leq \frac{2}{\omega_{\max }}\left(\sqrt{1+\xi_{\max }^{2}}-\xi_{\max }\right)
$$

being $\xi_{\max }$ the fraction of critical damping in the highest frequency mode. By default, Abaqus/Explicit introduces a certain bulk viscosity parameter which generates pressures proportional to the volumetric strain rate of the system and contributes to the energy dissipation from high frequencies.

In Abaqus/Explicit, two forms of bulk viscosity are implemented: linear (Eq.3.29) or quadratic (Eq.3.30) with the volumetric strain rate $\dot{\epsilon}_{\text {vol }}$.

$$
\begin{gathered}
p_{b v 1}=b_{1} \rho c_{d} L_{e} \dot{\epsilon}_{v o l} \\
p_{b v 2}=\rho\left(b_{2} L_{e} \dot{\epsilon}_{v o l}\right)^{2}
\end{gathered}
$$

This second form of viscous damping becomes relevant when dealing with high velocity gradients, which is the case of most impact environments. The fictitious bulk viscosity pressure opposes to the wavefront of the dilatational waves caused by loading and prevent solid elements to acquire zero volumes.

The contribution of the bulk viscosity damping to the critical time step (Eq.3.28) is element-dependent, each of them having a critical damping following the expression

$$
\xi=b_{1}-b_{2}^{2} \frac{L_{e}}{c_{d}} \min \left(0, \dot{\epsilon}_{v o l}\right)^{2}
$$

and the greatest value among the elements of the system will be the one implemented in the calculation of the stable increment (Eq.3.28). 


\subsection{Diagonal mass matrix}

One of the most relevant features of the explicit method is the employment of diagonal mass matrices, as the required procedure for solving the accelerations in the explicit scheme from Eq.3.32 consists of a simple division, whose computational cost is far lower than the required for the inversion of consistent mass matrices (defined by Eq.3.33).

$$
\begin{gathered}
\ddot{\mathbf{u}}^{i+1}=\mathbf{M}^{-1}\left(\mathbf{f}_{\text {ext }}^{i+1}+\mathbf{f}_{i n t}^{i+1}\right) \\
\mathbf{M}_{M N}=\int \rho \Phi_{M} \Phi_{N} \mathrm{~d} \Omega
\end{gathered}
$$

The diagonal mass matrix $\left(\mathbf{M}_{d(\Omega)}\right)_{M N}$ can be formulated by means of the Kronecher delta:

$$
\left(\mathbf{M}_{d(\Omega)}\right)_{M N}=\delta_{M N} \sum_{K} \int \rho \Phi_{M} \Phi_{K} \mathrm{~d} \Omega=\delta_{M N} \int \rho \Phi_{M} \mathrm{~d} \Omega
$$

There are several methods to obtain this lumped mass matrix, being the approach taken in Eq.3.34 the most simple, as a summation along rows or columns is performed. The suitability and convergence rates of diagonal mass matrices in the explicit FEM have been detailedly discussed in the work by $\mathrm{Wu}$ in 2006 [156], concluding that with relatively smooth solutions the same convergence rates in displacements and velocity than with consistent matrices can be obtained.

\subsection{Element controls}

FE codes for dynamic calculations often employ one-point integration due to its reduced computational cost, but this strategy requires the existence of certain controls when hourglassing modes arise (modes of zero energy). In fact, hourglassing is one of the biggest contributor to instabilities in FEA.

Several approaches exist to face this problem, depending on the element type. This section will focus on the eight-node hexahedron, as it will be 
posteriorly applied in the meshing for many impact simulations that will be discussed in this Thesis. Hourglass control becomes even more important when dealing with three-dimensional solid elements, as these modes can result in negative volumes and impossible configurations of the element (see Fig. $3.3)$.
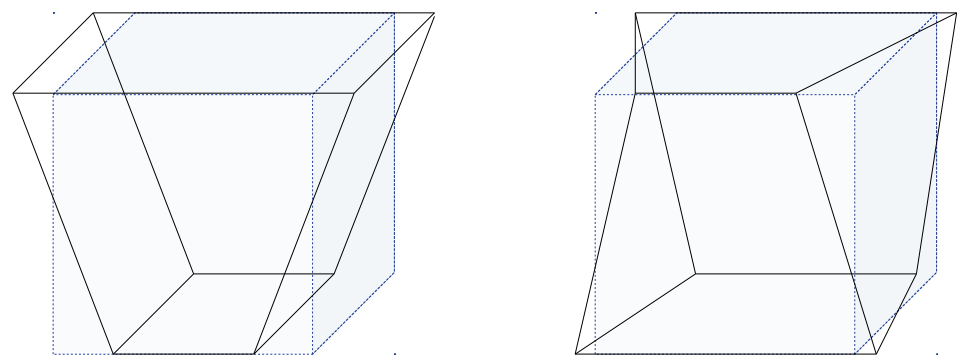

Figure 3.3: Different hourglass modes on 3D solid elements.

Belytschko and Bindeman (1993) [157] proposed a methodology for controlling the hourglass modes on the eight-node hexahedron by employing an assumed strain method and co-rotational coordinate systems. The purpose of an assumed strain field is to overcome the volumetric locking that incompressible or nearly incompressible materials, such as the brain, can experience during loading.

Some previous definitions for the notation are necessary in order to get to the fundamentals of the resolution for the hourglass problem proposed in [157]. From now on, $\mathbf{B}$ will be the matrix containing the derivatives of the shape functions of all nodes in the element. Taking into account an 8-node hexahedral element, the displacements of node $i$ can be expressed as:

$$
u_{i}=a_{0 i}+a_{1 i} x_{1}+a_{2 i} x_{2}+a_{3 i} x_{3}+c_{1 i} h_{1}+c_{2 i} h_{2}+c_{3 i} h_{3}+c_{4 i} h_{4}
$$

being $x_{1}, x_{2}, x_{3}$ the spatial coordinates and $h_{1}, \ldots, h_{4}$ the products of the element referential coordinates and the multipliers are arbitrary constants. 
By performing some transformations detailed in [158] the displacement gradient can be written as:

$$
u_{i, j}=\left(\hat{b}_{j}^{t}+h_{\alpha} \hat{\gamma}_{\alpha}^{t}\right) d_{i}
$$

where

$$
\hat{b}_{i}^{t}=\frac{1}{V} \int_{\Omega_{e}} N_{i}(\xi, \eta, \zeta) \mathrm{d} \Omega
$$

and therefore the resulting $\mathbf{B}$-matrix employing this notation has the following appearance:

$$
\hat{\mathbf{B}}=\left[\begin{array}{ccc}
\hat{b}_{x}^{t}+h_{\alpha, x} \hat{\gamma}_{\alpha}^{t} & 0 & 0 \\
0 & \hat{b}_{y}^{t}+h_{\alpha, y} \hat{\gamma}_{\alpha}^{t} & 0 \\
0 & 0 & \hat{b}_{z}^{t}+h_{\alpha, z} \hat{\gamma}_{\alpha}^{t} \\
\hat{b}_{y}^{t}+h_{\alpha, y} \hat{\gamma}_{\alpha}^{t} & \hat{b}_{x}^{t}+h_{\alpha, x} \hat{\gamma}_{\alpha}^{t} & 0 \\
\hat{b}_{z}^{t}+h_{\alpha, z} \hat{\gamma}_{\alpha}^{t} & 0 & \hat{b}_{x}^{t}+h_{\alpha, x} \hat{\gamma}_{\alpha}^{t} \\
0 & \hat{b}_{z}^{t}+h_{\alpha, z} \hat{\gamma}_{\alpha}^{t} & \hat{b}_{y}^{t}+h_{\alpha, y} \hat{\gamma}_{\alpha}^{t}
\end{array}\right]
$$

where the subscript $\alpha$ indicates the sum from 1 to 4 .

Back to the assumed strain field, its work principle is the following: in order to build the stiffness matrix, instead of directly employing the displacement gradient, it is projected onto the assumed strain field $\bar{\varepsilon}$ in such a way that $\bar{\varepsilon}=$ $\overline{\mathbf{B}} \mathbf{d}$, being $\mathbf{d}$ the displacement gradient vector $\left[\begin{array}{lll}d_{x} & d_{y} & d_{z}\end{array}\right]^{T}$ and the matrix $\overline{\mathbf{B}}$ is defined as the non-constant part of matrix $\mathbf{B}$ :

$$
\begin{array}{cc} 
& \overline{\mathbf{B}}=\hat{\mathbf{B}}_{c}+\overline{\mathbf{B}}_{n} \\
\hat{\mathbf{B}}_{c}=\left[\begin{array}{ccc}
\hat{b}_{x}^{t} & 0 & 0 \\
0 & \hat{b}_{y}^{t} & 0 \\
0 & 0 & \hat{b}_{z}^{t} \\
0 & \hat{b}_{z}^{t} & \hat{b}_{y}^{t} \\
\hat{b}_{z}^{t} & 0 & \hat{b}_{x}^{t} \\
\hat{b}_{y}^{t} & \hat{b}_{x}^{t} & 0
\end{array}\right] & \hat{\mathbf{B}}_{n}=\left[\begin{array}{ccc}
\hat{X}_{1234}^{t} & 0 & 0 \\
0 & \hat{Y}_{1234}^{t} & 0 \\
0 & 0 & \hat{Z}_{1234}^{t} \\
\hat{Y}_{1234}^{t} & \hat{X}_{1234}^{t} & 0 \\
\hat{Z}_{1234}^{t} & 0 & \hat{X}_{1234}^{t} \\
0 & \hat{Z}_{1234}^{t} & \hat{Y}_{1234}^{t}
\end{array}\right]
\end{array}
$$




$$
\overline{\mathbf{B}}_{n}=\left[\begin{array}{ccc}
\hat{X}_{1234}^{t} & -\bar{\nu} \hat{Y}_{3}^{t}-\nu \hat{Y}_{24}^{t} & -\bar{\nu} \hat{Z}_{2}^{t}-\nu \hat{Z}_{34}^{t} \\
-\bar{\nu} \hat{X}_{3}^{t}-\nu \hat{X}_{14}^{t} & \hat{Y}_{1234}^{t} & -\bar{\nu} \hat{Z}_{1}^{t}-\nu \hat{Y}_{34}^{t} \\
-\bar{\nu} \hat{X}_{2}^{t}-\nu \hat{X}_{14}^{t} & -\bar{\nu} \hat{Y}_{1}^{t}-\nu \hat{Y}_{24}^{t} & \hat{Z}_{1234}^{t} \\
\hat{Y}_{12}^{t} & \hat{X}_{12}^{t} & 0 \\
\hat{Z}_{13}^{t} & 0 & \hat{X}_{13}^{t} \\
0 & \hat{Z}_{23}^{t} & \hat{Y}_{23}^{t}
\end{array}\right] \quad \bar{\nu}=\frac{\nu}{(1-\nu)}
$$

The matrix $\overline{\mathbf{B}}_{n}$ is fundamental in this method as it contains the terms that control the avoidance of volumetric locking for nearly non-compressible materials. The highlighted terms in the matrix from Eq. 3.41 add an isochoric assumed strain field such that the hourglass mode $h_{4}$ is stabilised.

The whole purpose of the method is that the element stiffness matrix contains a stabilization stiffness matrix such that can counteract the effect of the "artificial" hourglass stiffness and fulfills the following condition:

$$
\mathbf{K}_{e}=\mathbf{K}_{c}+\mathbf{K}_{s t a b}=V \hat{\mathbf{B}}_{c}^{t} \mathbf{C} \hat{\mathbf{B}}_{c}+\int_{\Omega_{e}} \overline{\mathbf{B}}_{n}^{t} \mathbf{C} \overline{\mathbf{B}}_{n} \mathrm{~d} \Omega
$$

where $\mathrm{V}$ is the total volume of the element. The application of this method to an explicit code can be performed directly by incorporating the stabilization term as part of the vector of internal forces:

$$
\mathbf{f}^{i n t}=V \hat{\mathbf{B}}_{c}^{t} \sigma+\mathbf{f}^{s t a b}, \quad \mathbf{f}_{i}^{\text {stab }}=\sum_{\alpha=1}^{4} \mathbf{Q}_{i \alpha} \hat{\gamma}_{\alpha}^{t}
$$

which will lead to a modification of Step 3 in the general explicit scheme presented in Section 2.2, which will include a prior coordinate transformation to the co-rotational coordinate system and will end with the opposite transformation in order to obtain the internal force vector in the global coordinate system.

\subsection{Contact in explicit FEA}

Mechanical contact is the physical interaction between different bodies along their boundaries [159]. Since the 1970s, extensive research has been done in 
order to develop contact formulations applicable to the finite element analysis. Contact involves a series of constraint conditions which are often treated by means of variational approaches. Inside the latter, there are two main ways of adressing the contact problem: the penalty methods and the Lagrange multiplier methods [160].

The Lagrange multiplier method may ensure the condition of no-penetration between nodes but the solution of the system of equations is much more complex than with the penalty method, whose implementation is more simplistic.

\subsubsection{The Lagrange multipliers method}

To include contact interactions into a dynamic problem additional terms may be introduced in Eq.3.14. Defining a surface contact displacement constraint matrix $\mathbf{G}$ containing the restrictions in displacements, Eq.3.14 can be modified to

$$
\mathbf{M} \ddot{\mathbf{u}}+\mathbf{F}(\mathbf{u}, \dot{\mathbf{u}})+\mathbf{G}^{T} \lambda=\mathbf{R}
$$

The Lagrange multipliers are arranged in vector $\lambda$ and physically they represent the surface contact forces, which are unknowns. Besides, another condition should be satisfied:

$$
\mathbf{G}\{\mathbf{u}+\mathbf{X}\}=0
$$

where $\mathbf{u}$ is the displacement vector and $\mathbf{X}$ the material coordinate vector. The sum of them is the spatial coordinate vector.

Expressions 3.44 and 3.45 conform a system of equations whose unkowns are both the displacements and the vector $\lambda$. Its discretisation in time leads to:

$$
\begin{aligned}
\mathbf{M} \ddot{\mathbf{u}}_{n+1}+\mathbf{F}\left(\mathbf{u}_{n+1}, \dot{\mathbf{u}}_{n+1}\right)+\mathbf{G}_{n+1}^{T} \lambda_{n+1} & =\mathbf{R}_{n+1} \\
\mathbf{G}_{n+1}\left\{\mathbf{u}_{n+1}+\mathbf{X}\right\} & =0
\end{aligned}
$$

The last system of equations correspond to the contact constraints. As can be noticed from Eq.3.46, this method was initially intended for implicit schemes based on Newmark's integration. Posteriorly, Carpenter et al. (1991) [161] proposed a formulation of contact enforcement by means of Lagrange multipliers compatible with the explicit integration schemes which was named 
the forward increment Lagrange multiplier method. In this, displacement constraints at the current step are dependent on the Lagrange multipliers $\lambda$ at the previous step, resulting in the following equation of motion

$$
\begin{aligned}
\mathbf{M} \ddot{\mathbf{u}}_{n}+\mathbf{F}\left(\mathbf{u}_{n}, \dot{\mathbf{u}}_{n}\right)+\mathbf{G}_{n+1}^{T} \lambda_{n} & =\mathbf{R}_{n} \\
\mathbf{G}_{n+1}\left\{\mathbf{u}_{n+1}+\mathbf{X}\right\} & =0
\end{aligned}
$$

The procedure to solve this system based on the central difference method requires the splitting of the displacements in two components:

$$
\mathbf{u}_{n+1}=\mathbf{u}_{n+1}^{*}+\mathbf{u}_{n+1}^{c}
$$

First, all nodal displacements $\mathbf{u}_{n+1}^{*}$ are calculated for the current time step regardless of contact constraints by means of

$$
\mathbf{u}_{n+1}^{*}=h^{2} \mathbf{M}^{-1}\left(\mathbf{R}_{n}-\mathbf{F}\left(\mathbf{u}_{n}, \dot{\mathbf{u}}_{n}\right)\right)+2 \mathbf{u}_{n}-\mathbf{u}_{n-1}
$$

Once the displacement field is obtained, a search algorithm processes the current spatial coordinates given by $\mathbf{u}_{n+1}^{*}+\mathbf{X}$ and the nodes that have entered contact can be identified for the construction of matrix $\mathbf{G}_{n+1}$. Obtaining the resulting Lagrange multipliers vector is straightforward:

$$
\lambda_{n}=\left[h^{2} \mathbf{G}_{n+1} \mathbf{M}^{-1} \mathbf{G}_{n+1}^{T}\right]^{-1} \mathbf{G}_{n+1}\left(\mathbf{u}_{n+1}^{*}+\mathbf{X}\right)
$$

and finally the displacements associated to the nodes entering contact can be computed for their addition to Eq.3.48.

$$
\mathbf{u}_{n+1}^{c}=-h^{2} \mathbf{M}^{-1} \mathbf{G}_{n+1}^{T} \lambda_{n}
$$

The major benefit of this method is that the contact constraints are fully satisfied and the no-penetration condition is ensured. However, the main drawback of the Lagrange multiplier method is that the size of the system of equations is increased because of the incorporation of the multipliers as unknowns [160]. 


\subsubsection{The penalty method}

Penalty contact methods are also variational approaches of the contact problem, as it was the case with the Lagrange multipliers. Nonetheless, the treatment of the constraint conditions is different between both procedures. While with the Lagrange multipliers approach the no-penetration condition was satisfied as there is an exact constraint enforcement on contact surfaces, in the penalty method these constraints are not fully satisfied as it relies on the occurence of penetration.

Inside the explicit calculation schemes, however, the equation for Lagrange multipliers $\lambda$ can be satisfied at each time step but due to the central difference approach the equation of motion may not be exactly satisfied [156]. This means that the system is still not at equilibrium and the no-penetration cannot be ensured to be a final state, fact that diminishes the suitability of this contact approach inside an explicit calculation scheme. The penalty method, on the other hand, appears to fit better inside this context.

Physically, the penalty method can be regarded as the placement of springs of a certain stiffness between the surfaces susceptible to contact. The contact forces, therefore, instead of being considered unknowns of the problem (as it was the case in the multipliers method) are considered proportional to the amount of surface penetration [160]. This proportionality is introduced by means of the penalty parameter, whose choice determines the resulting accuracy of the contact calculation. The major benefit of this procedure is its implementation and calculation simplicity, as there are no additional unknowns in the final system of equations and the system matrix is positive definite [160].

As an example of implementation of the penalty method into an explicit scheme for nonlinear dynamic contact problems the work by Wu (2009) [162] is taken. Departing from Eq.3.11 and assuming contact can occur over a portion of the boundary $\Gamma_{c}$ the following formulation of the variational principle for the dynamic contact problem is achieved:

$$
\begin{array}{r}
\int_{\Omega}\left(\rho \ddot{u}_{i}\left(v_{i}-\dot{u}_{i}\right)+\sigma_{i j}\left(v_{i}-\dot{u}_{i, j}\right)\right) \mathrm{d} \Omega-\int_{\Gamma_{c}} \sigma_{i j} N_{i j}\left(v_{i}-\dot{u}_{i}\right) \mathrm{d} \Gamma= \\
\int_{\Omega} f_{i}\left(v_{i}-\dot{u}_{i}\right) \mathrm{d} \Omega+\int_{\Gamma_{s}} g_{i}\left(v_{i}-\dot{u}_{i}\right) \mathrm{d} \Gamma
\end{array}
$$


The next step is to introduce penalty terms into Eq.3.52. First, a penalty force should be created in order to compensate or "push" the points of the system that have penetrated an obstacle. This last condition is fulfilled when the velocity $\dot{u}_{N+}>0$ and the penalty force has the expression:

$$
\frac{1}{\varepsilon_{\nu}} \dot{u}_{N+} v_{N}
$$

Secondly, it is required to know the place where penetration has occured in order to properly apply the penalty force. Instead of employing an iterative search, a point-wise check of the penetration condition is performed along the entire $\Gamma_{c}$ domain based on the geometrical condition $(\mathbf{X}-\mathbf{Y}(\mathbf{X})) \cdot \mathbf{N}>0$. If $\mathbf{X}$ is any point belonging to $\Gamma_{c}, \mathbf{Y}(\mathbf{X})$ is the projection of this point along its normal on the obstacle surface. A penalty term is derived from this condition analogously to Eq.3.53:

$$
\frac{1}{\varepsilon_{x}}(\mathbf{X}-\mathbf{Y}(\mathbf{X}))_{N+} v_{N}
$$

Coefficients $\frac{1}{\varepsilon_{\nu}}$ and $\frac{1}{\varepsilon_{x}}$ represent viscosity and stiffness, respectively. Therefore, the inclusion of the penalty method on Eq.3.52 yields:

$$
\begin{array}{r}
\int_{\Omega}\left(\rho \ddot{u}_{i}\left(v_{\varepsilon}\right)_{i}+\sigma_{i j}\left(v_{\varepsilon}\right)_{i, j}\right) \mathrm{d} \Omega \\
+\int_{\Gamma_{c}}\left(\frac{1}{\varepsilon_{\nu}} \dot{u}_{N+}\left(v_{\varepsilon}\right)_{N}+\frac{1}{\varepsilon_{x}}(\mathbf{X}-\mathbf{Y}(\mathbf{X}))_{N+}\left(v_{\varepsilon}\right)_{N}-\sigma_{T i}\left(v_{\varepsilon}\right)_{T i}\right) \mathrm{d} \Gamma= \\
\int_{\Omega} f_{i}\left(v_{\varepsilon}\right)_{i} \mathrm{~d} \Omega+\int_{\Gamma_{s}} g_{i}\left(v_{\varepsilon}\right)_{i} \mathrm{~d} \Gamma
\end{array}
$$

Rearranging terms in order to obtain the second Newton's law in terms of the nodal shape functions and the lumped mass matrix, the nodal force vector can be identified as the sum of the internal and external forces plus the penalty force component.

$$
\begin{gathered}
\left(\mathbf{F}_{n}\right)_{i}^{M}=-\int_{\Omega}\left(\sigma_{i j} \Phi_{M, j}\right) \mathrm{d} \Omega+\int_{\Omega} f_{i} \Phi_{M} \mathrm{~d} \Omega+\int_{\Gamma_{s}} g_{i} \Phi_{M} \mathrm{~d} \Gamma+\mathbf{F}_{M i}^{\mathrm{P}} \\
\mathbf{F}_{M i}^{\mathrm{P}}=-\left(\frac{1}{\varepsilon_{v}}(\dot{u})_{N+}+\frac{1}{\varepsilon_{x}}\left(\mathbf{x}_{M}-\mathbf{y}_{M}\right)_{N+}\right)\left(\mathbf{N}_{x_{M}}\right)_{i} \\
-\left|\mathbf{F}\left(\mathbf{x}_{M}\right)_{t}\right|\left(\mathbf{t}_{x_{M}}\right)_{i}
\end{gathered}
$$


As it can be seen in the expression of the penalty force, it is dependent on the nodal velocities of the penetrating nodes. For this reason, this term is coupled to the explicit scheme after the step of the velocity update. This means that the accelerations are first computed without taking into account the contact forces. After that, the penetration condition $(\mathbf{X}-\mathbf{Y}) \cdot \mathbf{N}$ is evaluated at each node and then, once the penalty forces are calculated using Eq.3.57, the resulting motion variables are recomputed:

$$
\begin{gathered}
\mathbf{A}^{P}=\mathbf{M}^{-1} \mathbf{F}^{P}=\mathbf{M}^{-1}\left(\mathbf{F}_{N}^{P}+\mathbf{F}_{T}^{P}\right) \\
\mathbf{A}_{n}^{h}=\mathbf{A}_{n}^{h}+\mathbf{A}^{P} \quad \mathbf{V}_{n+\frac{1}{2}}^{h}=\mathbf{V}_{n+\frac{1}{2}}^{h}+\mathbf{A}^{P} \Delta t
\end{gathered}
$$

where $\mathbf{F}_{N}^{P}$ and $\mathbf{F}_{T}^{P}$ represent the normal and frictional forces in the onset of contact and $\mathbf{A}$ and $\mathbf{V}$ represent acceleration and velocity, respectively. If assuming a Coulomb friction model, the second term depends only on the first one by means of the friction coefficient $\left|\mathbf{F}_{T}^{P}\right| \leq \mu\left|\mathbf{F}_{N}^{P}\right|$.

In conjunction with the other variables of the explicit scheme, the contact forces are recalculated at each time increment. Given that the step size in explicit dynamic problems is small enough to ensure stability, the magnitude of the penetration does not normally reach high values and this prevents from sudden changes in the penalty forces [156].

The main problem that arises when employing the penalty contact formulation is the choice of proper penalty coefficients. Whereas in implicit analyses this selection is quite straightforward, it becomes an issue in dynamic problems as large values of the contact parameter can lead to excessive oscillations that may affect the global solution [160]. Wu [162] pointed out that, for high speed impact problems, better convergence is achieved when decreasing the values of the penalty coefficients. 


\section{Chapter 4}

\section{Impact behaviour of protective materials}

A major part of this Thesis has been devoted to the numerical study of the behaviour of personal protections and, more specifically, helmets, under impact conditions. The purpose is to predict the aftermaths that a ballistic impact can cause to a human head protected with a composite helmet.

To perform this study using Finite Element Analysis, three conditions need to be fulfilled:

- To develop a human head FE model capable of capturing the real behaviour of the different tissues under impact conditions.

- To develop a material model for the combat helmet that is able to represent the different composite damage mechanisms at the lowest computational cost as possible.

- To couple both models and recreate a ballistic impact environment.

This section will focus on the second bullet point. A methodology for the characterisation of composite plates under different impact conditions is presented. In order to finally achieve an accurate constitutive model representing the impact response of these panels, two experimental approaches have been taken: low-velocity impact tests and the Digital Image Correlation (DIC) technique. Five different impact energies have been tested in order to capture all possible failure mechanisms of the composite plate. 


\subsection{Overview}

In this section, damage caused on composite panels by low-energy impact is going to be addressed. This type of loading has the peculiarity that, even if no external observable damage can be attained, the low velocity impact can cause internal matrix cracking and delamination [163].

Several studies in the literature have conducted experimental low-velocity impact tests on composites (specifically CFRP) plates. Most of them have focused on the low impact energy range (up to 10-15J) [164, 165], in which many of the principal damage mechanisms can be observed: intralaminar fiber and matrix cracking and interlaminar delamination [164-166]. Nonetheless, many of the impact studies on CFRP plates employ unidirectional plies for their lay-up, whose effect is meant to be detrimental under such loading conditions. Instead, woven-fabric (WF) composites present a greater impact resistance as the transverse tensile strength is increased [167]. In fact, recent studies suggest that the predominant damage mechanisms under low-velocity impact differ from woven to non-woven composites $[168,169]$, being matrix cracking more relevant for the first and delamination for the second.

Naik et al. (2000) [167] compared different unidirectional and cross-ply laminates with WF laminates and reported a lower in-plane failure function for the latter, which was claimed to be the cause of their improved impact resistance. Ullah et al. (2012) [170] studied the impact behaviour of woven CFRPs by means of experimental pendulum tests at energy levels up to 2 J. Their specimens were four-layered laminates constituted by woven carbon fibers on a thermoplastic polyurethane polymer matrix. The authors reported an energy level of $0.6 \mathrm{~J}$ as sufficient to cause sample fractures whereas impact energies of $0.5 \mathrm{~J}$ led to non-significant damage initiation. Greater impact energy values were considered in the experimental work by Murat and Rahman (2017) [171] on woven CFRP plates. Matrix microcracks were detected by means of SEM at an energy level of $1 \mathrm{~J}$, whereas fibre breakage required at least $5 \mathrm{~J}$.

Regarding constitutive modeling of composite plates, Zhang et al. (2010) [172] proposed the use of continuum damage mechanics (CDM) theory as a tool to analyse progressive damage. Its main principle is that the occurrence of micro damage and micro cracks in the composite material are constitutively translated to a loss of stiffness of the material. It is considered a suitable method for determining both damage initiation and evolution in composite 
materials. Because of this continuous degradation of the material properties, convergence problems in the FE analysis may arise. For this reason, explicit algorithms are preferred for this type of low velocity problems. Furthermore, proper numerical study of the impact damage on composite materials should include these non-linearities and also the dynamic effects of the problem [163]. This approach, which has been extensively used in several FE studies on composite plates undergoing impact $[164,165,170,173,174]$, will be adopted for the numerical simulation of the low-velocity tests conducted in this study.

\subsection{Experimental tests on composite plates}

In this section the experimental impact testing of composite plates will be presented. The specimens employed for the tests are woven (twill) carbon fiber panels with an epoxy matrix of an average size of 10x16 cm. The panel lay-up consists of 10 woven layers, constituting a total thickness of the plate of $2.1 \mathrm{~mm}$.

The test apparatus is a Charpy pendulum Ceast ${ }^{\circledR} 6545$ upon which a modification has been performed in order to enhance blunt impact testing of non-notched samples. To that purpose, a spherical anvil has been mounted on the pendulum, placed in alignment with the center of mass of the system. Additionally, the base that holds the samples has been modified in order to support specimens of greater dimensions than the ones commonly employed for Charpy testing. Two lateral supports have been built to maintain the sample plates in a vertical position while constraining the movement on two of the plate's edges. Both horizontal and vertical positions of the plate can be adjusted in order to ensure a centered perpendicular impact of the spherical anvil. These vertical grips have been painted matt black to avoid possible reflections of the aluminum structure on the sample under the high-illumination conditions required to record with a high-speed camera. The overall setup for the experimental tests is illustrated in Fig. 4.1.

After preliminary testing, it was observed that negligible damage was sustained by the sample when the angle of the pendulum with respect to the vertical was lower than $90^{\circ}$. Angles greater than $90^{\circ}$ led to minor plastic damage on the impacted surface and the apparition of cracks on the outer layers and delamination. Nonetheless, complete failure was only achieved when impacting the plate five times. 


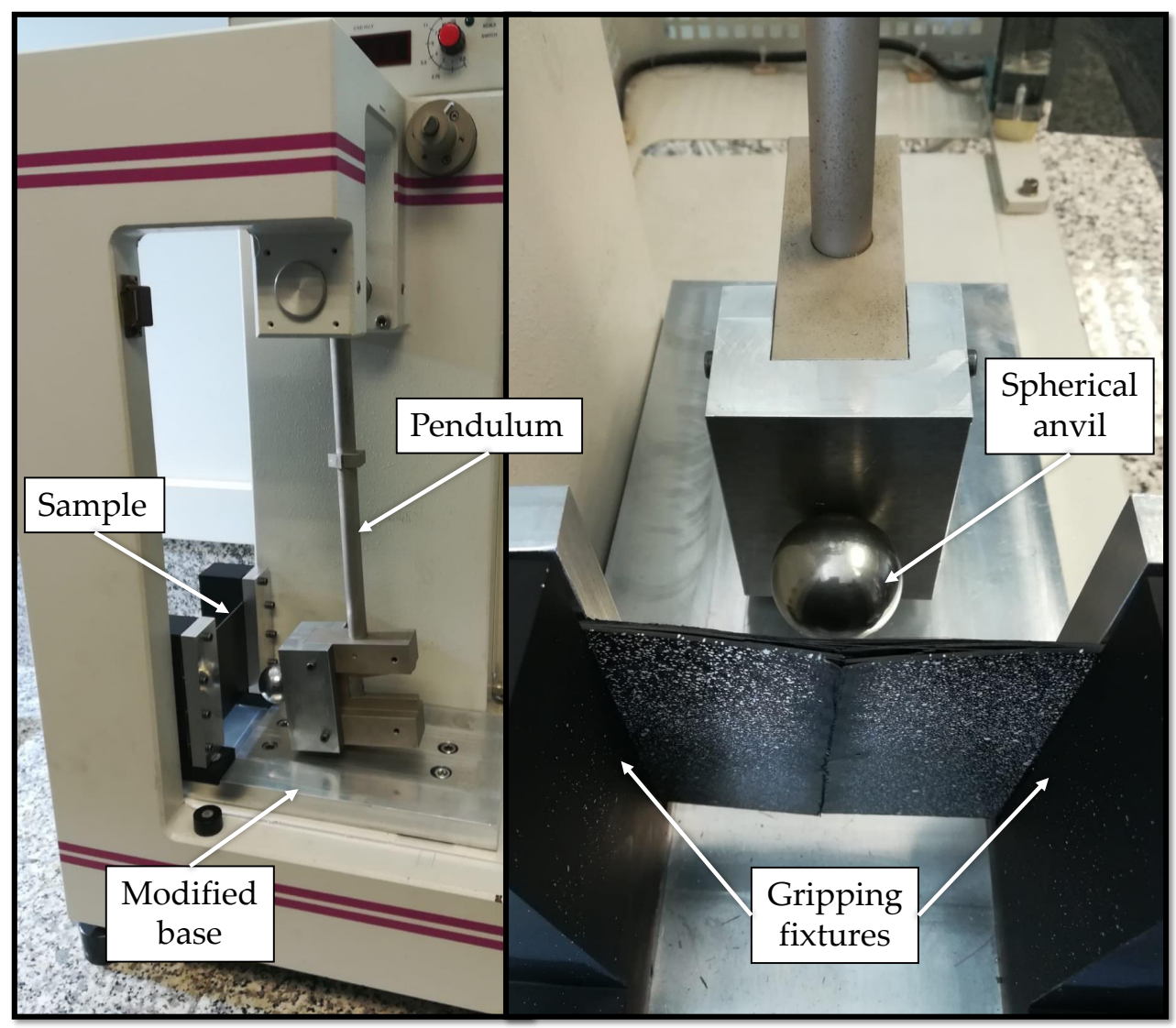

Figure 4.1: Experimental setup for blunt impact tests on composite samples.

All the impact tests were performed releasing the pendulum from its maximum angle with respect to its equilibrium position $\left(175^{\circ}\right)$. From this initial position, the velocity of the pendulum head at the impact stage is $3.7 \mathrm{~ms}^{-1}$. In order to relate the main damage phenomena of CFRP panels with the magnitude of the impact suffered, the mass of the pendulum has been varied by adding or removing weight attachments. As a result, five initial kinetic energies have been tested: 23.61, 29.09, 33.98, 57.84 and $68.11 \mathrm{~J}$. The matrix of experimental tests performed is deployed in Table 4.1.

The energy absorption of the panels has been computed by means of the rebounding velocity of the pendulum, which is measured employing medium- 
speed image acquisitions with a camera placed perpendicular to the pendulum's plane.

\begin{tabular}{llll}
\hline $\begin{array}{l}\text { Pendulum } \\
\text { mass }(\mathrm{kg})\end{array}$ & $\begin{array}{l}\text { Number of } \\
\text { samples }\end{array}$ & $\begin{array}{l}\text { Velocity } \\
\left(\mathrm{ms}^{-1}\right)\end{array}$ & $\begin{array}{l}\text { Kinetic } \\
\text { Energy }(\mathrm{J})\end{array}$ \\
\hline 3.45 & 2 & 3.7 & 23.61 \\
4.25 & 2 & 3.7 & 29.09 \\
4.95 & 3 & 3.7 & 33.98 \\
8.45 & 1 & 3.7 & 57.84 \\
9.95 & 1 & 3.7 & 68.11 \\
\hline
\end{tabular}

Table 4.1: Experimental test matrix for the pendulum blunt impact on CFRP plates.

\subsubsection{Experimental results}

The absorbed energy calculated for each panel and the damage features visible to the naked eye on each of their external surfaces have been summarised in Table 4.2. The energy absorbed by the plates in the two cases with greater impact energies could not be measured due to set-up limitations but will be predicted from posterior FE modeling.

Delfosse and Poursartip [175] reported similar values for the energy absorbed by CFRP plates subjected to drop tower impacts. The plate dimensions in [175] are very similar to the ones employed in the experimental tests presented in this section except for the thickness value, which is approximately twice as thick $(4.65 \mathrm{~mm})$ in the work by Delfosse and Poursartip. Three of their tests were performed at impact energies of 30,34 and $58 \mathrm{~J}$, which are essentially comparable to the cases presented here of $29.09,33.98$ and $57.84 \mathrm{~J}$. It can be seen that for impact energies of 30 and $34 \mathrm{~J}$ the energy absorbed by the plates in [175] (16 J and $27 \mathrm{~J}$, respectively) is about the same magnitude of the values reported here (14.68 J and $23.22 \mathrm{~J})$. Woven CFRP of similar thickness to the ones employed in [175] were drop-tower tested by Grasso et al. [169] at a maximum energy level of $28.80 \mathrm{~J}$. Their reported $E_{a b s}$ at this loading condition was $13.12 \mathrm{~J}$, slightly lower than that obtained in the present study but still in the same range. 


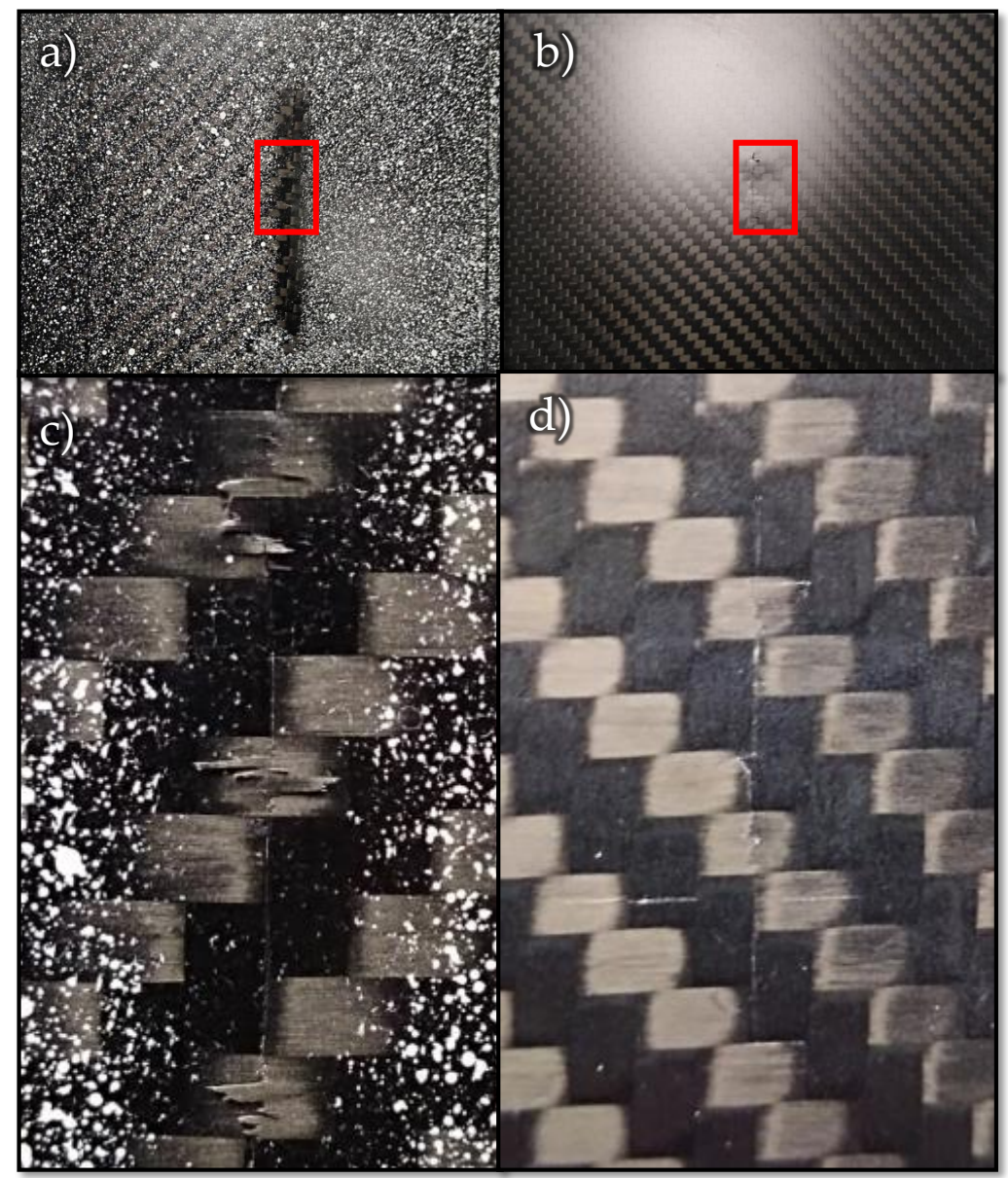

Figure 4.2: External (a) and striking surface (b) of a plate tested at an impact energy of 29 J. Superficial damage at the exterior (c) and interior layers (d).

For the lowest impact energy group, no visible damage is achieved in the external surface of the plate and a minimal clivage is detected in the surface in contact with the anvil. Impact energies of 29J do lead to macroscopic failure, as illustrated in Fig. 4.2. Comparing the appearance of the external surface (a) and the striking surface (b) of a tested sample at this energy level, it is noticeable that in the latter, a small circular shaped-region is damaged in contrast to the longitudinal crack that characterises the failure in the external layer. Fig. 4.2 (d) is a detailed view of the permanent indentation left in the 
striking plate surface. It can be distinguished a longitudinal vertical crack of approximately $20 \mathrm{~mm}$ length initiated at the first contact point of the plate with the sphere. Beyond this starting point, failure propagates through the transition regions between the fill and warp bundles and causes breakage of the fibers oriented horizontally. On the opposite face of the plate (Fig. 4.2 (c)) the failure trend is analogous with a larger extent of broken fibres and a final crack length of $50 \mathrm{~mm}$.

\begin{tabular}{|c|c|c|c|}
\hline $\begin{array}{l}\text { Kinetic } \\
\text { Energy } \\
(\mathrm{J})\end{array}$ & $\begin{array}{l}\text { Absorbed } \\
\text { Energy } \\
(\mathrm{J})\end{array}$ & External surface damage & Striking surface damage \\
\hline 23.61 & 11.15 & No visible damage & $\begin{array}{l}\text { Minor permanent indenta- } \\
\text { tion. }\end{array}$ \\
\hline 29.09 & 14.68 & $\begin{array}{l}\text { Superficial vertical crack of } \\
50 \mathrm{~mm} \text { in length with fibre } \\
\text { breakage in warp direction. }\end{array}$ & $\begin{array}{l}\text { Permanent indentation of } 20 \\
\text { mm diameter. Minor longi- } \\
\text { tudinal crack confined in this } \\
\text { area. }\end{array}$ \\
\hline 33.98 & 23.22 & $\begin{array}{l}\text { Vertical crack of } 60 \mathrm{~mm} \text { in } \\
\text { length with fibre breakage in } \\
\text { warp direction. }\end{array}$ & $\begin{array}{l}\text { Permanent indentation of } 20 \\
\text { mm diameter. Longitudinal } \\
\text { crack confined in this area. }\end{array}$ \\
\hline 57.84 & - & $\begin{array}{l}\text { Complete vertical through- } \\
\text { thickness failure of } 55 \mathrm{~mm}\end{array}$ & $\begin{array}{l}\text { Slight delamination of the in- } \\
\text { ner layers with crack initia- } \\
\text { tion on one free edge. }\end{array}$ \\
\hline 68.10 & - & $\begin{array}{l}\text { Full-height longitudinal fail- } \\
\text { ure. Delamination at the } \\
\text { free edges. }\end{array}$ & $\begin{array}{l}\text { Permanent deformation and } \\
\text { shear failure in the contact } \\
\text { area. }\end{array}$ \\
\hline
\end{tabular}

Table 4.2: Summary of the experimental results from the impact tests.

A certain increase in energy, up to $34 \mathrm{~J}$, results in the triggering of other visible failure modes than the ones achieved at $29 \mathrm{~J}$. Whereas fibre breakage in the $29 \mathrm{~J}$ case was confined to the most external layer, at the next energy level the fibrous lattice is damaged in half of the plies in the through-thickness direction. At the interface between the last ply broken due to bending and the first unbroken one an interlaminar delamination is found. 


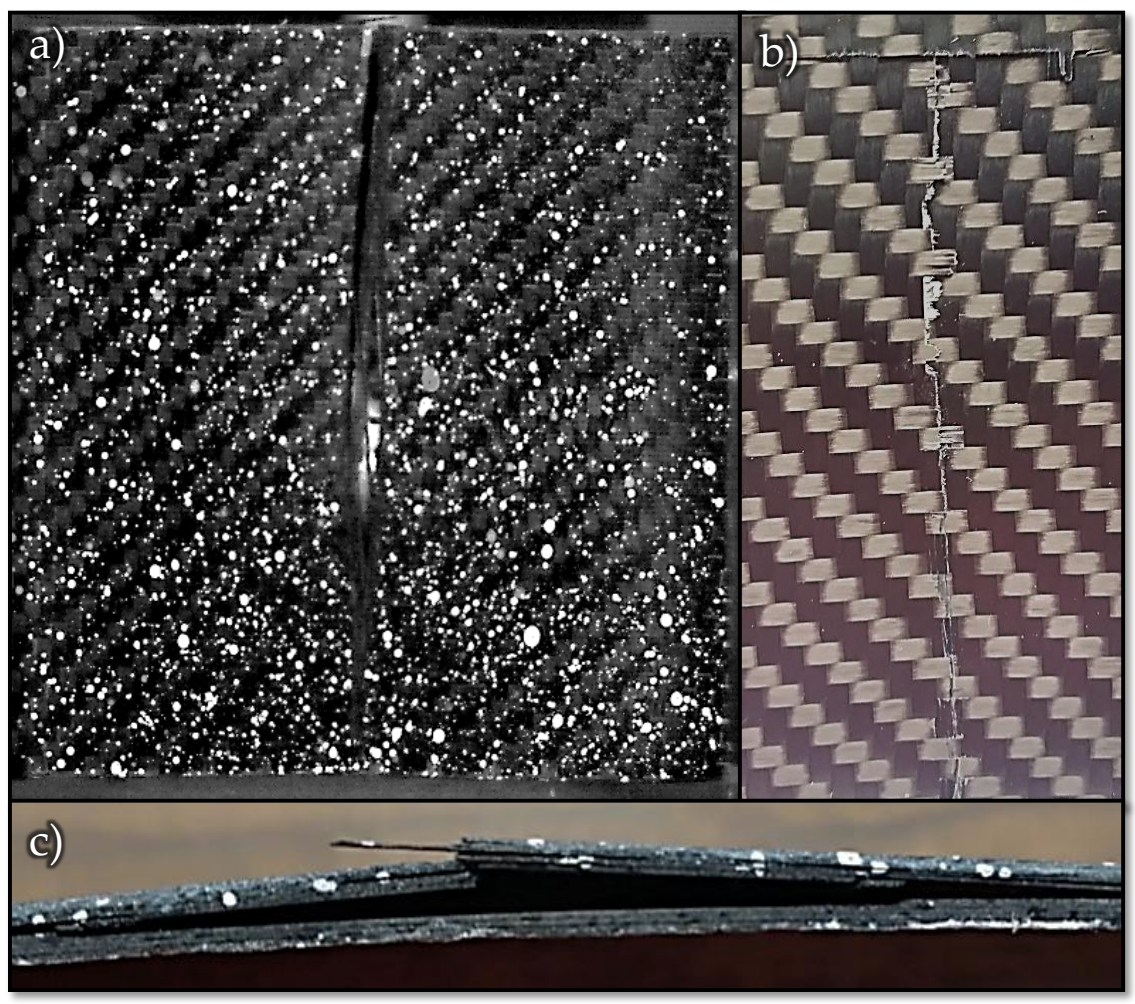

Figure 4.3: External (a) and striking surface (b) of a plate tested at an impact energy of $68.10 \mathrm{~J}$. (c) Detail of the delamination at the free edge.

Increasing the impact energy results in the increase of damaged and failed regions. For instance, full-height failure of the external layer is achieved at an impact energy of $68 \mathrm{~J}$. As it can be observed in Fig. 4.3 (a), the spherical anvil penetrates the central region of the plate and its two lateral halves debond completely. The affected area decreases in thickness towards the free edges of the plate.

\subsection{High-speed camera measurement}

In order to obtain a quantification of the displacement fields along the composite samples during impact, the experimental procedure defined in the previous section has been recorded using a high-speed camera Photron Fastam Mini 
UX100. The device was placed perpendicular to the sample surface in order to allow posterior image postprocessing. A capture rate of 8000 fps was selected to acquire the images of the impacts as it proved to be sufficient to detect initiation and evolution of the damage at the external face of the plate without compromising the resolution of the image files.
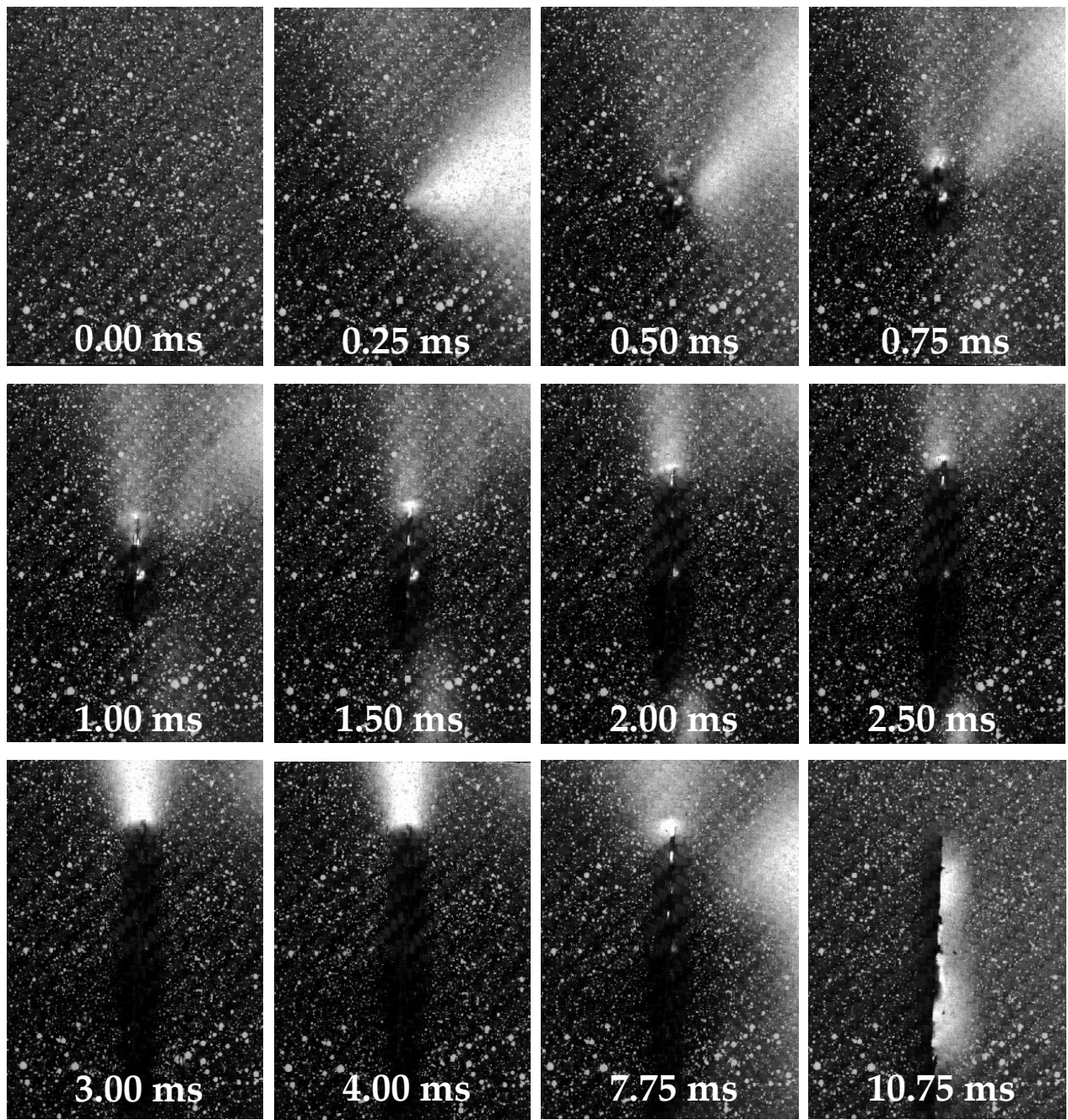

Figure 4.4: Time sequence of crack growth during impact recorded with highspeed camera at a frame rate of $8000 \mathrm{fps}$. 
One issue that arises when dealing with high-speed recording is the progressive darkening of the scene as the frame rate is increased. This is caused by the aperture of the lens. Therefore, for capturing instantaneous events there is a high need of an optimal intense illumination of the area to film. Two spotlights equipped with cloth diffusers were employed to avoid excessive light reflection on the sample surface during loading.

One of the sequences of the image recording is illustrated in Fig. 4.4. It is observable how the out-of-plane displacements can generate brightness changes in the pictures. This does not constitute a major issue if the purpose of the acquisition is to simply identify fracture propagation at a macroscopical level. Nonetheless, corrected illumination protocols have been adopted in order to obtain images as uniform in brightness as possible in order to enhance further image correlation.

\subsubsection{Digital Image Correlation (DIC)}

After the acquisition of the test images from the high-speed camera, these have been processed by using a Digital Image Correlation (DIC) software. Specifically, the commercial software Vic-2D has been employed.

DIC is a non-invasive measurement technique based on the correlation between specific sets of points belonging to images that present different loading states of a certain specimen. Commonly, these points for correlation are obtained from a randomly distributed speckle along the sample. Because of its apparent simplicity, the DIC technique has been extensively used as an experimental procedure which complements the results from traditional strain/motion-measurement devices. Even though originally employed for other purposes such as feature recognition, microscopy or medicine, some of the first DIC applications for mechanics took place in 1982 from the hand of Peters and Ranson [176]. Posteriorly, the first image correlation algorithms which gave birth to what today is known as 2D-DIC were presented by Sutton et al. (1983) [177]. Some works from the literature have directly applied this technique to the assessment of the mechanical response of composite materials. For instance, Bouvet et al. (2012) [164] visualised and quantified the permanent indentation on CFRP plates after impact using three-dimensional DIC (based on stereography). This methodology was also employed in a recent work by Tuo et al. (2019) [165] to characterise out-of-plane displacements in CFRP panels during compression after impact (CAI). 


\section{Fundamentals of image correlation}

As DIC algorithms are based on the matching of specific patterns within images, a key point in order to achieve a good correlation is the application of a randomly, widely distributed speckle pattern. Normally it is painted over the surface of the tested specimen and therefore it moves and deforms in conjunction with it. Its randomness is relevant as it ensures a unique solution when the subset (or window containing a region from the reference pattern) iterates through the whole region of interest (ROI) looking for intensity correspondence. For this same reason, homogeneous plane surfaces are also not desirable as there are not distinguishable features for pattern matching [178].

Considering a intensity distribution of a certain object as $G(x, t)$ which varies unidimensionally with time, the simplest approach for motion estimation assuming small displacements will be based on small differences. Therefore, $G(x, t)$ can be approximated as a first-order Taylor series:

$$
G(x+\Delta x, t)=G(x, t)+\frac{\partial G}{\partial x} \Delta x
$$

If considering a constant velocity along $x, \dot{u}$, the previous expression can be reformulated as

$$
\Delta G=G(x-\Delta x, t)-G(x, t) \quad \Delta x=\dot{u} \Delta t
$$

After performing simple correspondences and appliying the limit $\Delta t \rightarrow 0$, the following expression is achieved:

$$
\frac{\partial G}{\partial t}+\dot{u} \frac{\partial G}{\partial x}=0
$$

The extension of Eq. 4.3 to the two-dimensional case results in the homogeneous system of differential equations:

$$
\frac{\partial G}{\partial t}+\mathbf{v} \cdot \nabla G=0
$$

Eq. 4.4 can be discretised and therefore be applied to a number $N$ of particular image points along a specific domain. The resulting over-determined system from Eq.4.6 can be solved using a least-squares approach for the average motion $\Delta \overline{\mathbf{x}}$ of this set of N-points from one image to another (Eq.4.7).

$$
\Delta \mathbf{x} \cdot \nabla G=-\Delta G \rightarrow \mathbf{G} \Delta \overline{\mathbf{x}}=-\mathbf{g}
$$




$$
\begin{gathered}
{\left[\begin{array}{cc}
\frac{\partial G^{1}}{\partial x} & \frac{\partial G^{1}}{\partial y} \\
\frac{\partial G^{2}}{\partial x} & \frac{\partial G^{2}}{\partial y} \\
\vdots & \vdots \\
\frac{\partial G^{N}}{\partial x} & \frac{\partial G^{N}}{\partial y}
\end{array}\right]\left[\begin{array}{c}
\Delta \bar{x} \\
\Delta \bar{y}
\end{array}\right]=-\left[\begin{array}{c}
\Delta G^{1} \\
\Delta G^{2} \\
\vdots \\
\Delta G^{N}
\end{array}\right]} \\
{\left[\begin{array}{c}
\Delta \bar{x} \\
\Delta \bar{y}
\end{array}\right]=-\left[\begin{array}{cc}
\sum\left(\frac{\partial G}{\partial x}\right)^{2} & \sum\left(\frac{\partial G}{\partial x} \frac{\partial G}{\partial y}\right) \\
\sum\left(\frac{\partial G}{\partial x} \frac{\partial G}{\partial y}\right) & \sum\left(\frac{\partial G}{\partial y}\right)^{2}
\end{array}\right]^{-1}\left[\begin{array}{l}
\sum \frac{\partial G}{\partial x} \Delta g \\
\sum \frac{\partial G}{\partial y} \Delta g
\end{array}\right]}
\end{gathered}
$$

Nonetheless, as based on a first-order Taylor series expansion, this differential method is intended for problems of small displacements. This, and other limitations of the presented method were overcome with the introduction of the Lucas-Kanade algorithm [179], which keeps the mathematical simplicity of Eq. 4.7, with the system to solve being the following:

$$
\left[\begin{array}{c}
\Delta x \\
\Delta y
\end{array}\right]=\left[\begin{array}{cc}
\sum\left(\frac{\partial G}{\partial x}\right)^{2} & \sum\left(\frac{\partial G}{\partial x} \frac{\partial G}{\partial y}\right) \\
\sum\left(\frac{\partial G}{\partial x} \frac{\partial G}{\partial y}\right) & \sum\left(\frac{\partial G}{\partial y}\right)^{2}
\end{array}\right]^{-1}\left[\begin{array}{c}
\sum \frac{\partial G}{\partial x}(F-G) \\
\sum \frac{\partial G}{\partial y}(F-G)
\end{array}\right]
$$

Functions $F$ and $G$ from the last term of Eq. 4.8 are the key features of this method. $F$ is a subset taken from an image, and acts as a template that is to be found in the image after displacement, $G$. It means, $F$ will iterate along $G$ until it finds the position in $G$ where the grey value difference between them is the minimum, i.e. the region of $G$ that best resembles the template $F$. For this reason this method is named Template Matching and its objective is to find a displacement vector $\overline{\mathbf{d}}$ such that minimises the term $\sum|G(\mathbf{x}+\overline{\mathbf{d}})-F(\mathbf{x})|^{2}$.

\section{Performance of the correlation}

\section{- Region of interest (ROI)}

The region of interest (ROI) is a window contained in the speckled images upon which the correlation computation will be performed. The ROI should enclose an area with proper speckle distribution and free of singularities (such as holes or surface changes). 


\section{- Subset and step size}

These two parameters are fundamental in the template matching method upon which DIC is based. The subset determines the dimensions (in pixels) of the template patch $F$ (following the notation of the Lucas-Kanade description) and therefore the size of each area that grids the ROI. The optimal magnitude of the subset depends on the problem type, but it has always to be sufficient to gather a representative piece of the region to analyse. This means, a certain number of speckle points should be enclosed in the subset in order to avoid false template matches.

The step parameter determines the spatial increment of the displacement vector at each iteration. This means, given a step value of 1 , the subset $G(\mathbf{x}+\overline{\mathbf{d}})$ to be compared with the template $F(\mathbf{x})$ will shift one pixel to the right at the next iteration. It implies that correlation will be performed over all the pixels in the ROI. Intuitively, smaller steps comprise less correlation error, but also the computational expense is increased by a power of 2 .

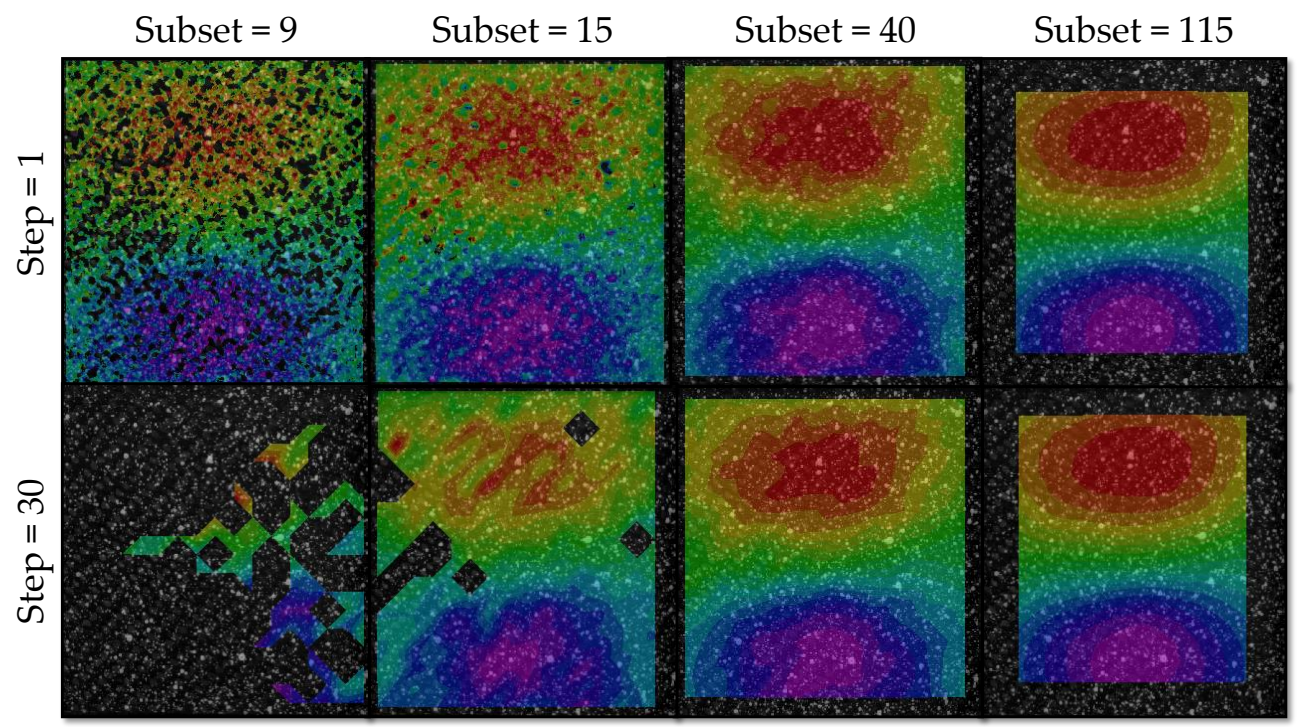

Figure 4.5: Variation of the step and subset values and its influence on DIC using Vic 2D.

Fig. 4.5 represents the influence of these two parameters in obtaining a 
good correlation. The contour plot shown is the displacement field in the vertical direction (in the image plane) at the beginning of impact. A subset size of 9 pixels, taking into account the speckle size of the plate, only encloses one or two speckle points and therefore after convergence there appear areas where the correlation was not achieved (that are visible in Fig. 4.5 as black regions embedded in the contour plot). Increasing subset sizes diminishes this particle-like appearance of the contour plots and make them more uniform and with better defined boundaries. The influence of the step size is noticeable for relatively low subset values, whereas it does not really affect the solution substantially for subset sizes greater than 40 pixels.

\section{- Correlation criteria}

As it was introduced when explaining the high-speed image acquisition, sudden changes in lighting in the region of interest between frames affect the success of the subset correlation, as the intensity values of the pattern may have changed and it is therefore no further recognisable. To overcome this undesired event there are different methods, or correlation criteria, to minimise the effect of brightness changes.

The simplest and least time consuming criterion is the squared differences. Nonetheless, it is sensitive to any changes in the intensity levels due to lighting and only accurate for uniform-brightness problems. Another criterion, which is the employed by default in Vic 2D, is the normalized squared differences. In the latter, changes in light intensity are supported if they are quantifiable by a scale factor with respect to the reference pattern. Finally, the last criteria implemented in Vic 2D is the zero-normalized squared differences, which is the most insensitive of the three to light changes as it takes into account both proportional (scale) and independent (offset) lighting shifts.

Fig. 4.6 shows the performance of these correlation criteria when applied to the images acquired from the Charpy impact tests. The frame taken as an example corresponds to the beginning of the impact, where the center of the plate bends outwards and the right side quarter of the image (highlighted in white in Fig. 4.6) presents greater reflectivity. Therefore, this region is the most susceptible to underachieve correlation, as it is the case when applying the squared differences and the normalized squared differences criteria (Fig. 4.6 (left) and (center), respectively). It can be observed that several pixels within this area fail to converge and a discontinuity in the results arises. Nonetheless, when choosing the zero-normalized squared difference criterion, 
pattern matching is achieved in the whole ROI.
Squared differences
Normalized squared differences
Zero-normalized squared differences

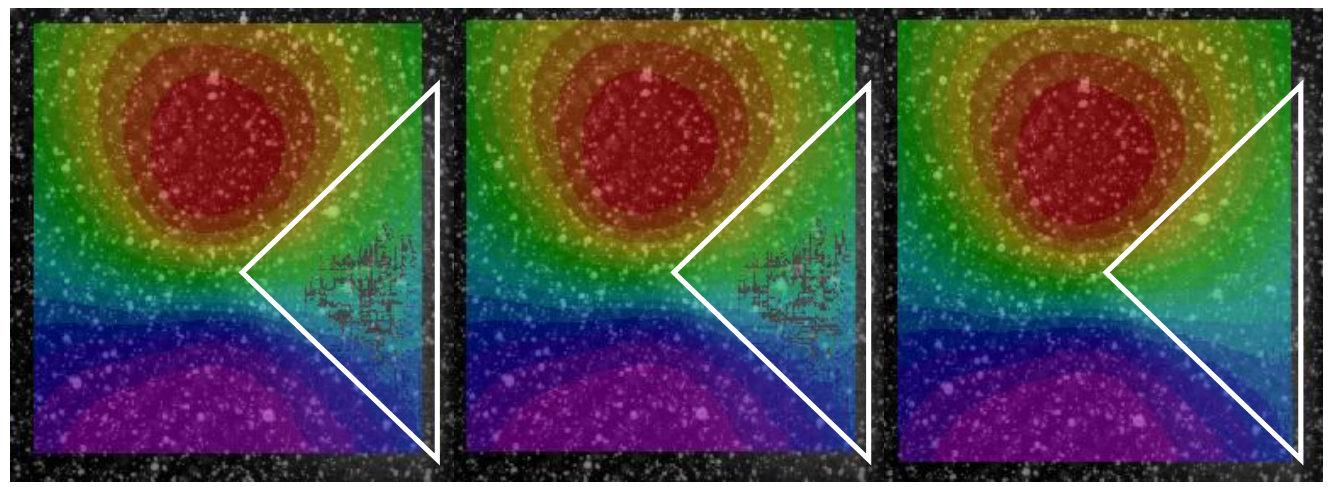

Figure 4.6: Output displacement fields employing three different correlation criteria in Vic 2D.

\subsection{FE explicit simulation}

\subsubsection{Model description}

A numerical replication of the experimental impact tests has been carried out in the finite element software Abaqus/Explicit. The experiment has been simplified to its two main parts: the composite plate and the spherical anvil.

The modeling approach adopted for the CFRP panel is based on its mesoscale. This means that the main constituents (fibers and matrix) are not explicitly modeled as separated materials but gathered together in homogenised solid elements. The associated computational expense is dramatically reduced and the correspondence with experimental tests leads to high correlations, as presented in previous studies on composites submitted to impact $[174,180$ 182]. At the same time, it is necessary a certain discretisation along the thickness direction of the plate, as the inter-ply interaction plays a relevant role in energy absorption and structural integrity. As a result, the composite 
plate has been modeled as a 10-layered solid of a total thickness of $2 \mathrm{~mm}$. It has been meshed with reduced integration 8-node hexahedra (type C3D8R in Abaqus) reaching a total of 295360 elements. The mesh is refined in the center of the plate, the area where the impact will take place. The spherical anvil has been simplified to a hemisphere in order to save computational expense, and is meshed with the same element type as the plate. In order to be consistent with the dynamics of the experimental test, the mass set to the hemispherical anvil is the equivalent of the whole pendulum system. Fig. 4.7 illustrates the complete model for the Charpy test simulation.

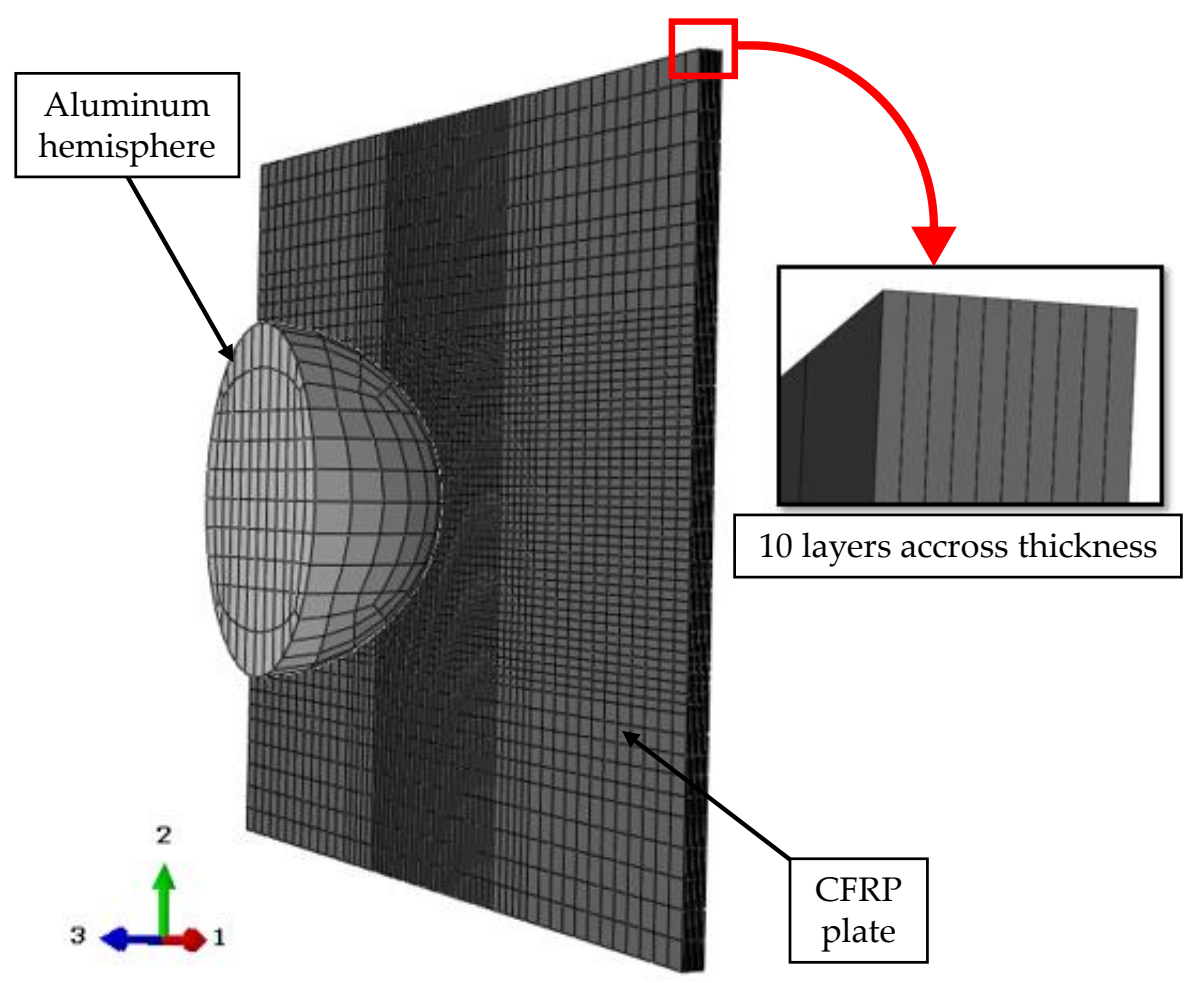

Figure 4.7: Abaqus/Explicit setup of the impact tests. 


\section{Prescribed boundary conditions}

The composite plate is constrained on two of its edges, restricting the movement in the impact direction. An initial velocity value, whose magnitude is calculated from the drop in potential energy of the pendulum during testing, is set to the hemisphere. This velocity has a constant value of $3.7 \mathrm{~ms}^{-1}$ in all the simulated cases whereas the impactor mass is varied in order to achieve the different experimental impact energies.

A general contact algorithm is chosen to simulate the interaction between the plate and the anvil, whereas a specific surface-to-surface interaction is set between the adjacent composite plies of the plate. This allows the formulation of a cohesive behaviour between layers, that enhances the modeling of the delamination phenomenon. Details on this specific interaction will be presented in the next section that deals with material modeling. On the other hand, a friction coefficient of 0.25 has been set for the interaction between the hemispherical anvil and the plate [165]. In all the simulations performed, the artificial strain energies, which are a measure of the extent of hourglass being experienced in the model, are monitored and kept to a maximum level of the $3 \%$.

\section{Material properties}

An orthotropic material behaviour has been chosen to model the CFRP plate, with the material constants summarised in Table 4.4. Fiber and matrix failure are implemented to the material by means of a VUMAT subroutine (following the scheme presented in Fig. 4.8) based on Hashin's failure criteria for fabrics. Six damage modes are evaluated, four corresponding to fiber damage and two regarding matrix failure:

- Fiber tensile failure in direction $1, \sigma_{1}>0$ :

$$
d_{f t 1}=\left(\frac{\sigma_{1}}{X_{1 t}}\right)^{2}+\left(\frac{\tau_{12}}{S_{12}}\right)^{2}+\left(\frac{\tau_{13}}{S_{13}}\right)^{2}
$$

- Fiber compressive failure in direction $1, \sigma_{1}<0$ :

$$
d_{f c 1}=\left(\frac{\sigma_{1}}{X_{1 c}}\right)^{2}+\left(\frac{\tau_{12}}{S_{12}}\right)^{2}+\left(\frac{\tau_{13}}{S_{13}}\right)^{2}
$$


- Fiber tensile failure in direction $2, \sigma_{2}>0$ :

$$
d_{f t 2}=\left(\frac{\sigma_{2}}{X_{2 t}}\right)^{2}+\left(\frac{\tau_{12}}{S_{12}}\right)^{2}+\left(\frac{\tau_{23}}{S_{23}}\right)^{2}
$$

- Fiber compressive failure in direction $2, \sigma_{2}<0$ :

$$
d_{f c 2}=\left(\frac{\sigma_{2}}{X_{2 c}}\right)^{2}+\left(\frac{\tau_{12}}{S_{12}}\right)^{2}+\left(\frac{\tau_{23}}{S_{23}}\right)^{2}
$$

- Matrix tensile failure, $\sigma_{3}>0$ :

$$
d_{m t}=\left(\frac{\sigma_{3}}{X_{3 t}}\right)^{2}+\left(\frac{\tau_{12}}{S_{12}}\right)^{2}+\left(\frac{\tau_{13}}{S_{13}}\right)^{2}+\left(\frac{\tau_{23}}{S_{23}}\right)^{2}
$$

- Matrix compressive failure, $\sigma_{3}<0$ :

$$
d_{m c}=\left(\frac{\sigma_{3}}{X_{3 c}}\right)^{2}+\left(\frac{\tau_{12}}{S_{12}}\right)^{2}+\left(\frac{\tau_{13}}{S_{13}}\right)^{2}+\left(\frac{\tau_{23}}{S_{23}}\right)^{2}
$$

A scheme of the functioning of the VUMAT subroutine inside the explicit analysis is illustrated in Fig. 4.8. At each iteration, the stress tensor of the material is computed and the failure subcriteria contained in the VUMAT script are evaluated. If any of the damage modes has been reached, the software performs a degradation of the stiffness matrix of the material by means of the damage parameter $d$ corresponding to the failed mode. When the stress in an element has exceeded the threshold value for fracture, the element is deleted from the simulation and is not included in the algorithm for further iterations. 


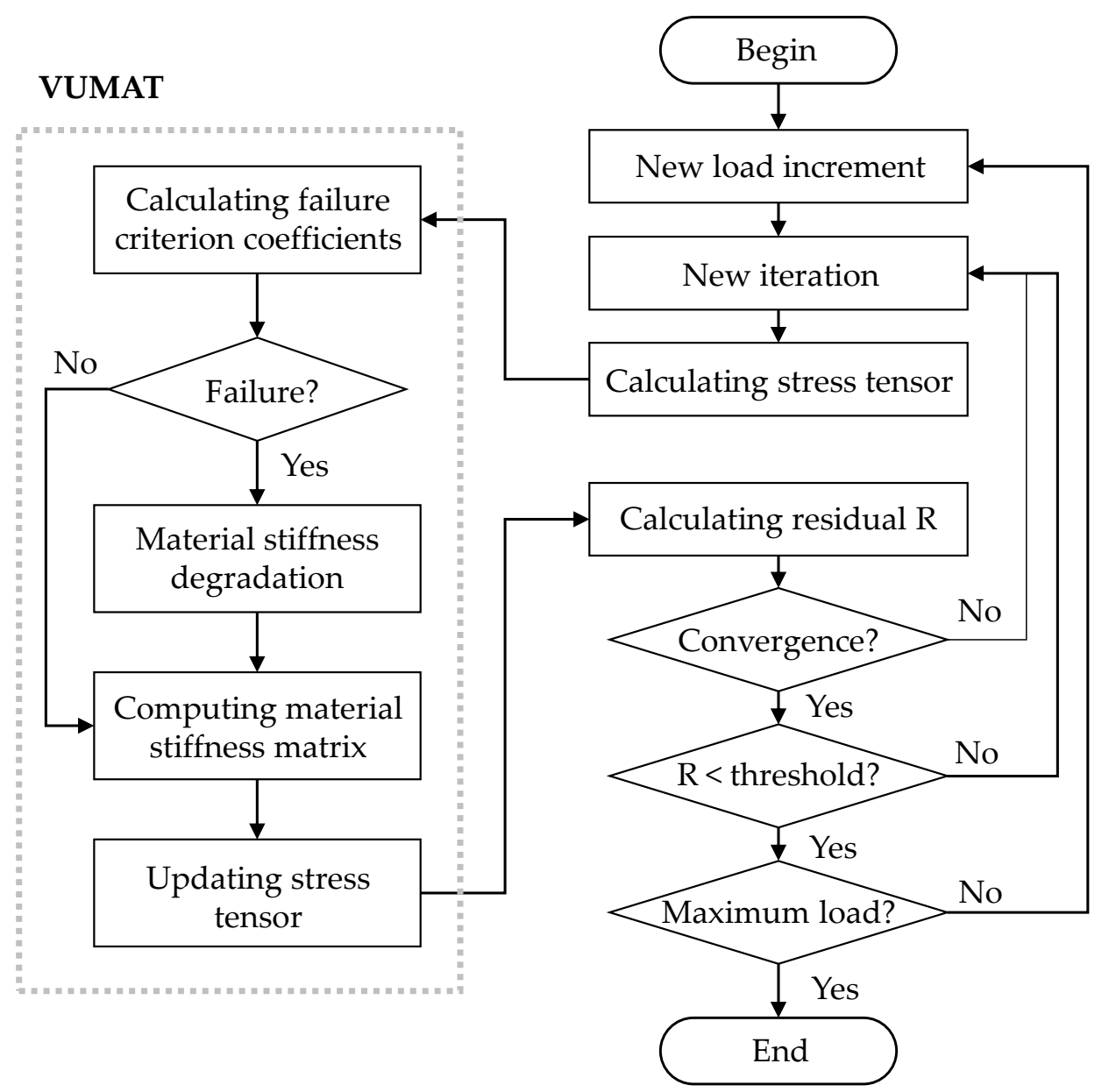

Figure 4.8: Diagram of an explicit procedure including a VUMAT subroutine for damage evolution. Extracted from Wang et al. (2014) [183].

\subsubsection{Interlaminar damage using CZM}

Interlaminar damage is included in the model through the contact interaction of cohesive surfaces previously mentioned. In Abaqus/Explicit, this type of interaction assumes a linear elastic traction-separation law until the initiation of damage, and then the cohesive stiffness at the interfaces is degraded following a specified path until a limit on which the contacting nodes between 
adjoining surfaces separate and thus the cohesive bond breaks.

Damage initiation has been characterised by means of a quadratic stress criterion, whose expression is the following:

$$
\left\{\frac{\left\langle t_{n}\right\rangle}{t_{n}^{0}}\right\}^{2}+\left\{\frac{t_{s}}{t_{s}^{0}}\right\}^{2}+\left\{\frac{t_{t}}{t_{t}^{0}}\right\}^{2}=1
$$

where $t_{n}, t_{s}$ and $t_{t}$ are the normal and the two shear tractions of the nominal traction stress vector [184]. $t_{n}^{0}, t_{s}^{0}$ and $t_{t}^{0}$ are the peak stress values in each direction at which damage initiates.

The degradation of the cohesive stiffness has been characterised with an exponential law whose independent variable is the energy released once damage has initiated. A mixed-mode evolution is chosen, which is based on the relative proportions of normal and shear stresses:

$$
m_{1}=\frac{G_{n}}{G_{T}} \quad m_{2}=\frac{G_{s}}{G_{T}} \quad m_{3}=\frac{G_{t}}{G_{T}}
$$

being $G_{T}=G_{n}+G_{s}+G_{t}$. To define complete failure, the energy dissipated in failure $G^{C}$ is employed. In the case of a mixed-mode damage evolution, this critical value is computed from the fracture toughness of each of the three modes. Specifically, the expression for $G^{C}$ selected for the cohesive interaction in the CFRP plate corresponds to the Benzeggagh-Kenane (BK) criterion:

$$
G^{C}=G_{n}^{C}+\left(G_{s}^{C}-G_{n}^{C}\right)\left\{\frac{G_{s}+G_{t}}{G_{T}}\right\}^{\eta}
$$

A graphical representation of the mixed-mode interaction is found in Fig. 4.9. The shear mode considered here accounts for the contribution of modes II and III: $G_{s h}=G_{s}+G_{t}, \tau^{0}=\sqrt{\left(t_{s}^{0}\right)^{2}+\left(t_{t}^{0}\right)^{2}}$. The normal and shear modes conform the boundaries if the traction-separation behaviour and the whole cohesive response is mapped based on the damage initiation law (the quadratic stress criterion) and the damage evolution law (BK). In the case illustrated in Fig. 4.9, cohesive stiffness degradation is considered as a linear function for simplicity. Nonetheless, an exponential damage evolution law will be employed in the material model of the CFRP plate. 


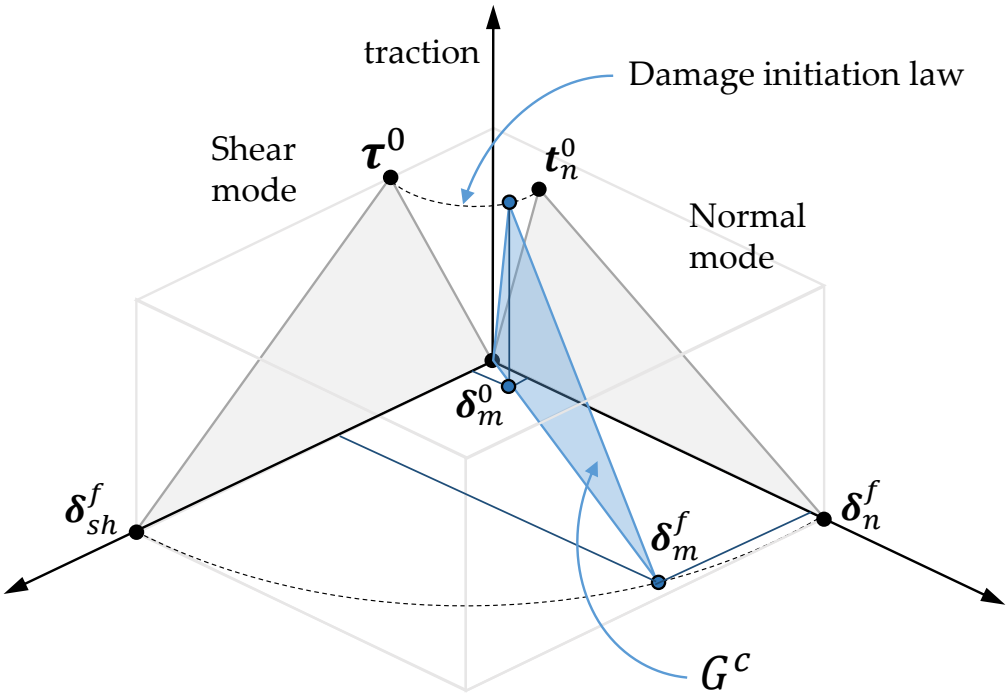

Figure 4.9: Graphical representation of the mixed-mode cohesive interaction implemented in Abaqus/Explicit.

The material parameters to characterise interlaminar damage in the CFRP plate in Abaqus/Explicit have been extracted from Tuo et al. [165] and are gathered in Table 4.3.

\begin{tabular}{cccc}
\hline \multicolumn{4}{c}{ Damage initiation } \\
\hline Criterion & $t_{n}^{0}(\mathrm{MPa})$ & $t_{s}^{0}(\mathrm{MPa})$ & $t_{t}^{0}(\mathrm{MPa})$ \\
\hline Quad. stress & 30 & 80 & 80 \\
\hline \multicolumn{4}{c}{ Damage evolution } \\
\hline Criterion & $G_{n}^{C}\left(\mathrm{Jm}^{-2}\right)$ & $G_{s}^{C}\left(\mathrm{Jm}^{-2}\right)$ & $G_{t}^{C}\left(\mathrm{Jm}^{-2}\right)$ \\
\hline Energy & 560 & 920 & 920 \\
\hline
\end{tabular}

Table 4.3: Mechanical parameters set to model the cohesive interaction between the composite plies. 
Elastic properties

\begin{tabular}{cccccc}
\hline$E_{11}(\mathrm{MPa})$ & $E_{22}(\mathrm{MPa})$ & $E_{33}(\mathrm{MPa})$ & $\nu_{12}$ & $\nu_{13}$ & $\nu_{23}$ \\
\hline 53100 & 53100 & 8000 & 0.074 & 0.055 & 0.074 \\
& & & & \\
$G_{12}(\mathrm{MPa})$ & $G_{13}(\mathrm{MPa})$ & & $G_{23}(\mathrm{MPa})$ & \\
\hline 3700 & 3700 & & 3790 & \\
\hline
\end{tabular}

Strength properties

\begin{tabular}{cccccc}
\hline$X_{11}(\mathrm{MPa})$ & $X_{22}(\mathrm{MPa})$ & $X_{33}(\mathrm{MPa})$ & $S_{12}(\mathrm{MPa})$ & $S_{13}(\mathrm{MPa})$ & $S_{23}(\mathrm{MPa})$ \\
\hline 565 & 565 & 420 & 66 & 66 & 66 \\
\hline
\end{tabular}

Table 4.4: Material properties applied to the woven CFRP plies of the plate in the numerical model.

\subsubsection{Results}

The objective of the FE reconstruction of the Charpy tests is to achieve an optimal set of mechanical properties such that the CFRP behaviour in the simulation matches the experimental. The latter has been characterised in terms of the energy absorbed by the plate (computed as the difference in kinetic energy in the pendulum), the macroscopic damage and the measurement of the strain fields sustained at the external surface at the first stage of impact (using high-speed recording and DIC). A comparison of the numerical and experimental outcomes for each of these features is presented along this section.

\section{Absorbed energy}

The achievement of an accurate numerical response in terms of energy dissipated from impact is conditioned by a large number of user-defined parameters such as constraints or contact definitions. Above all, the constitutive and damage laws implemented to the CFRP plate play the major role in the energy 
absorption. $E_{a b s}$ includes the contribution of the energy stored elastically, the energy dissipated in fibre and matrix breakage, and the energy from delamination. There is also some additional dissipation due to vibrations and friction, but its magnitude is quasi-negligible with respect to the other terms.
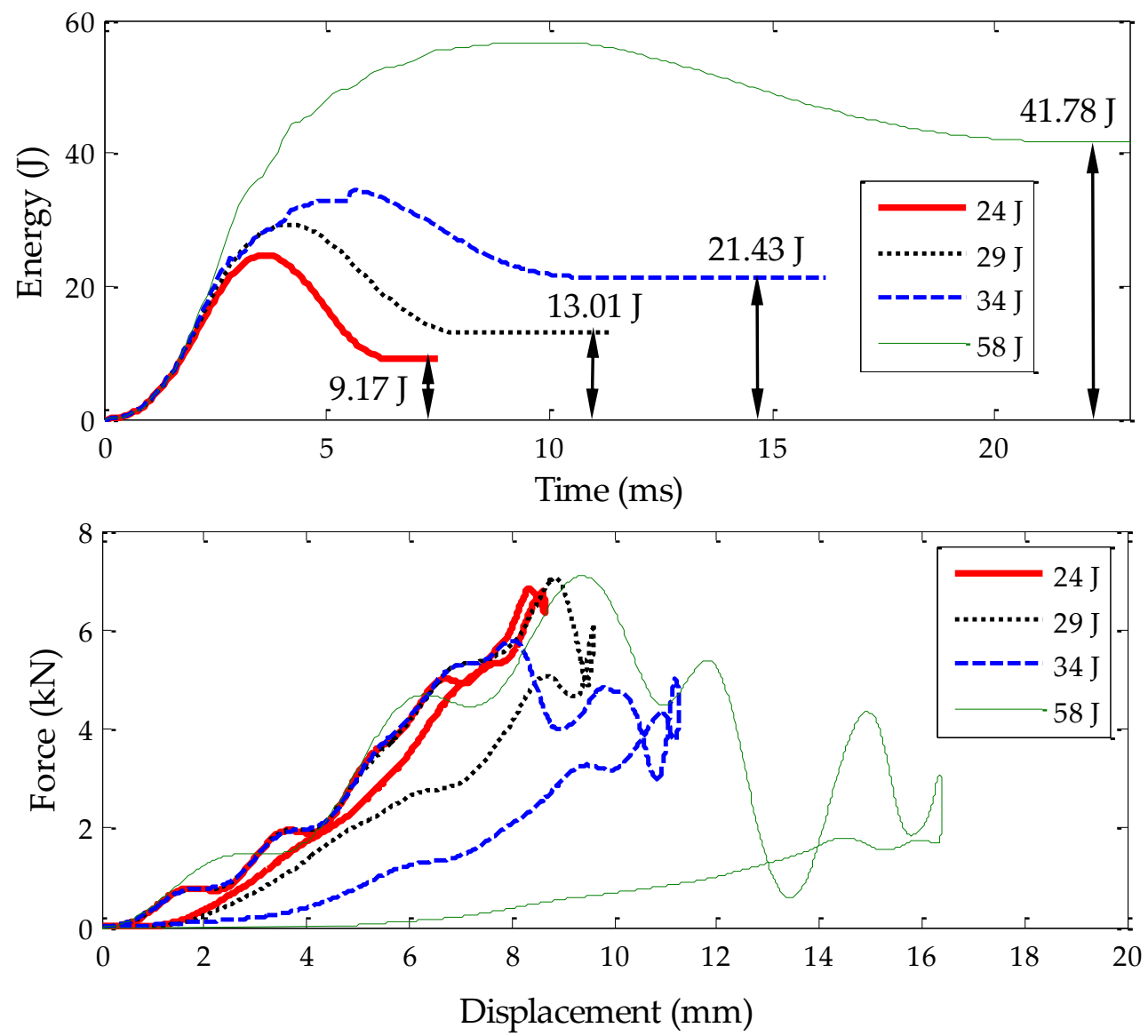

Figure 4.10: Absorbed energy $E_{a b s}$-time and force-displacement curves for the simulated impacts on the CFRP plate.

The time evolution in energy of the composite plates during impact has been extracted from the numerical simulation of each input energy. These curves are gathered in Fig.4.10 (top). All of them present the same trends, 
which are consistent with those reported in the literature on low-velocity impact at CFRP plates [165, 169, 185]. During the first stage of impact (approximately until $1.5 \mathrm{~ms}$ ), all the curves collapse forming a toe region that coincides with the beginning of contact. After that, there is a linear rise in the energy values, whose slope depends on the input impact energy, that terminates when this later value is reached. At this point of maximum energy in the plate both the elastic and damage contributions are present. This maximum is followed by a linear decrease whose rate becomes slower as the plate results more damaged. If the plate has undergone severe damage, this linear part of the curve is shorter or inexistent. Finally, once all the elastic energy stored in the composite plate has been transferred to the impactor for its rebound, the energy-time curve reveals a plateau that stabilises at a determined value, which is the impact energy absorbed by the plate.

Comparing the values of $E_{a b s}$ for each energy level obtained with FE simulation and experimentally, it can be observed that the first tends to underpredict this parameter for the lower energy cases. For impact energies of 24 and $29 \mathrm{~J}$ the numerically predicted $E_{a b s}$ are 9.17 and $13.01 \mathrm{~J}$, respectively. These values are a $17.7 \%$ and $11.38 \%$ lower than that measured from the impact tests. Nonetheless, this difference becomes $7 \%$ for the impact energy level of $34 \mathrm{~J}$, showing a decrease in the error of the numerical prediction as the input energy of the impact delivered to the plate increases. The percentage of absorbed energy increases with the input magnitude as there is more dissipation in the damage mechanisms. For the $24 \mathrm{~J}$ case, most of this energy is stored elastically by the plate and only a $39 \%$ is dissipated through delamination. Nonetheless, when additional failure modes such as fiber and matrix cracking arise, the extent of absorbed energy reaches the $72 \%$ (for the $58 \mathrm{~J}$ case).

The force-displacement curves measured for the impactor are represented in Fig.4.10 (bottom) for the different energies. Results from the simulation of the experimental test at $68 \mathrm{~J}$ have been omitted as there is partial penetration of the impactor through the plate, leading to excessive maximum displacements. Peak force values are in agreement with experimental and numerical tests reported in the literature on similar impact conditions (Bouvet et al. [164] obtained a peak force of $8 \mathrm{kN}$ for a $25 \mathrm{~J}$ impact). All the represented cases reveal a maximum force of about $7 \mathrm{kN}$ which corresponds to impactor displacements around $8.5 \mathrm{~mm}$. The combined experimental and numerical work by Lopes et al. [180] reports peak forces in the range of $6-7 \mathrm{kN}$ for impact energies from 9.1 to $29.7 \mathrm{~J}$. The area enclosed by the force-displacement 
curves increases with the energy of impact, analogously to the absorbed energy. For the lowest impact energies, the loading and unloading of the plate follow almost the same path as the elastic properties of the material remain mostly undamaged. Conversely, at higher energies, these properties are partially or completely degraded and the unloading response is less stiff.

\section{Damage in the CFRP plates}

It has been observed in the previous section that energy dissipation during impact is enhanced by the activation of the damage modes in the plate. A second step to evaluate the validity of the developed numerical model is to compare the macroscopic damage and failure sustained by the plate at the different energy levels with the patterns obtained experimentally. Both interlaminar and intralaminar damage mechanisms will be addressed.

An example of the evolution of delamination with time is illustrated in Fig. 4.11. The case taken for demonstration corresponds to an impact energy of $34 \mathrm{~J}$, but interlaminar damage evolution follows the same pattern for all the impact cases simulated. During the first stage of contact between the anvil and the plate, the first ply of the striking surface experiences interface stresses overpassing the damage initiation threshold in the region just above the contact area (represented in red in Fig. 4.11 (top)). Bouvet et al. [164] observed this same behaviour and claimed that the beginning of delamination was mainly dependent on mode I. As the bending stresses increase in the plate, interlaminar damage expands axially, towards the non-impacted surface of the plate, and transversally, leading to a damaged region confined above the impactor. It is clearly noticeable in Fig. 4.11 that the middle section of the plate remains undamaged, forming the delamination cone reported in other studies [164] that is meant to concentrate matrix cracks caused by out-of-plane stresses. This behaviour has been observed in all the impacts simulated in this study, taking place during the first stages of contact.

The evolution of cohesive damage and failure appears to be dependent on the impact energy in view of the results obtained. In the cases of impact energies of $34 \mathrm{~J}$ or higher, when the tensile stresses at the non-struck surface approach the fiber tensile strength value, sudden cohesive damage initiation is found in the interface between the most external (from the impact site) layers. Conversely, for lower impact energies there is not fiber failure at the 
non-striking layers and the delaminated area grows after the impacted ply leading to complete detachment along the vertical symmetry axis of the plate.
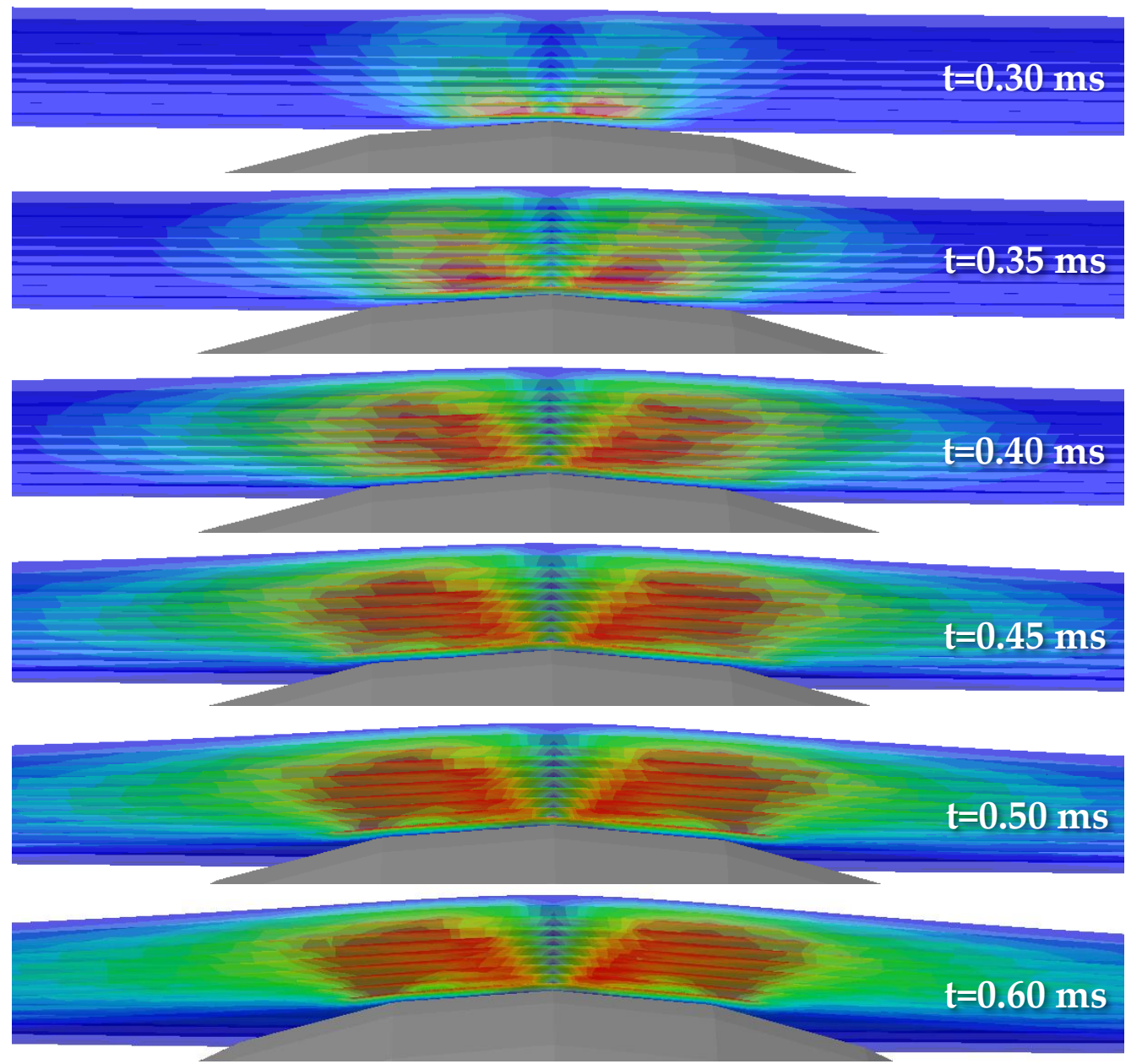

Figure 4.11: Simulated interlaminar mixed-mode failure in the composite plates. Red-colored regions indicate that damage between plies is initiated. The illustrated sequence corresponds to the $34 \mathrm{~J}$ case.

Intralaminar damage has also been characterised in the numerical simulations. Apart from discussing its contribution to the energy balance in the 
previous section, macroscopic failure in the plate plies has been examined and compared to the experimental results. Fig.4.12 shows the impacted and nonimpacted surfaces of the CFRP after impact attenuation obtained in the FE analysis.

For the lowest impact energy $(23.61 \mathrm{~J})$ the external surface remains undamaged, as obtained experimentally, while the impacted site presents a permanent indentation of about $20 \mathrm{~mm}$ of diameter. This indentation is also present in the physical tested plate, but this presents also a small and very superficial crack that could not be predicted with the numerical model maybe due to the element size at the impact region and the variation of the material properties considered. At the next impact energy level (29.09 J) superficial cracks are found in the external and internal plies both in the experimental tests and in the numerical simulations. This finding means that in the impact energy interval between 23.61 to $29.09 \mathrm{~J}$ there is a threshold beyond which fibre breakage is enhanced.

When the impact energy is $33.98 \mathrm{~J}$, a good agreement between the numerical and experimental macroscopical damage is achieved at both surfaces of the plate. At the non-impacted surface, a $50 \mathrm{~mm}$ vertical crack grows from the center of the plate, and a combination of permanent indentation and fiber cracking is observable at the impacted surface, being the indented region deeper and wider than the corresponding to the impact of $23.61 \mathrm{~J}$.

For the highest energy level $(68.11 \mathrm{~J})$ a vertical crack is developed along the full height of the plate. Approximatedly half of this crack propagates through the entire thickness, being this fractured area more noticeable at the impact site. Bending stresses cause fiber breakage at both the inner and outer surfaces of the plate and after impact the tested samples reveal a permanent deflection. Fiber failure in the striked surface does not extend vertically across the full plate, as the free ends remain unbroken. Conversely, these regions on the upper and lower edges of the plate are highly delaminated, achieving complete detachment between adjacent layers at the mid-thickness. This propagation of the delaminated area towards the plate edges is also observable for an impact energy of $58 \mathrm{~J}$. 


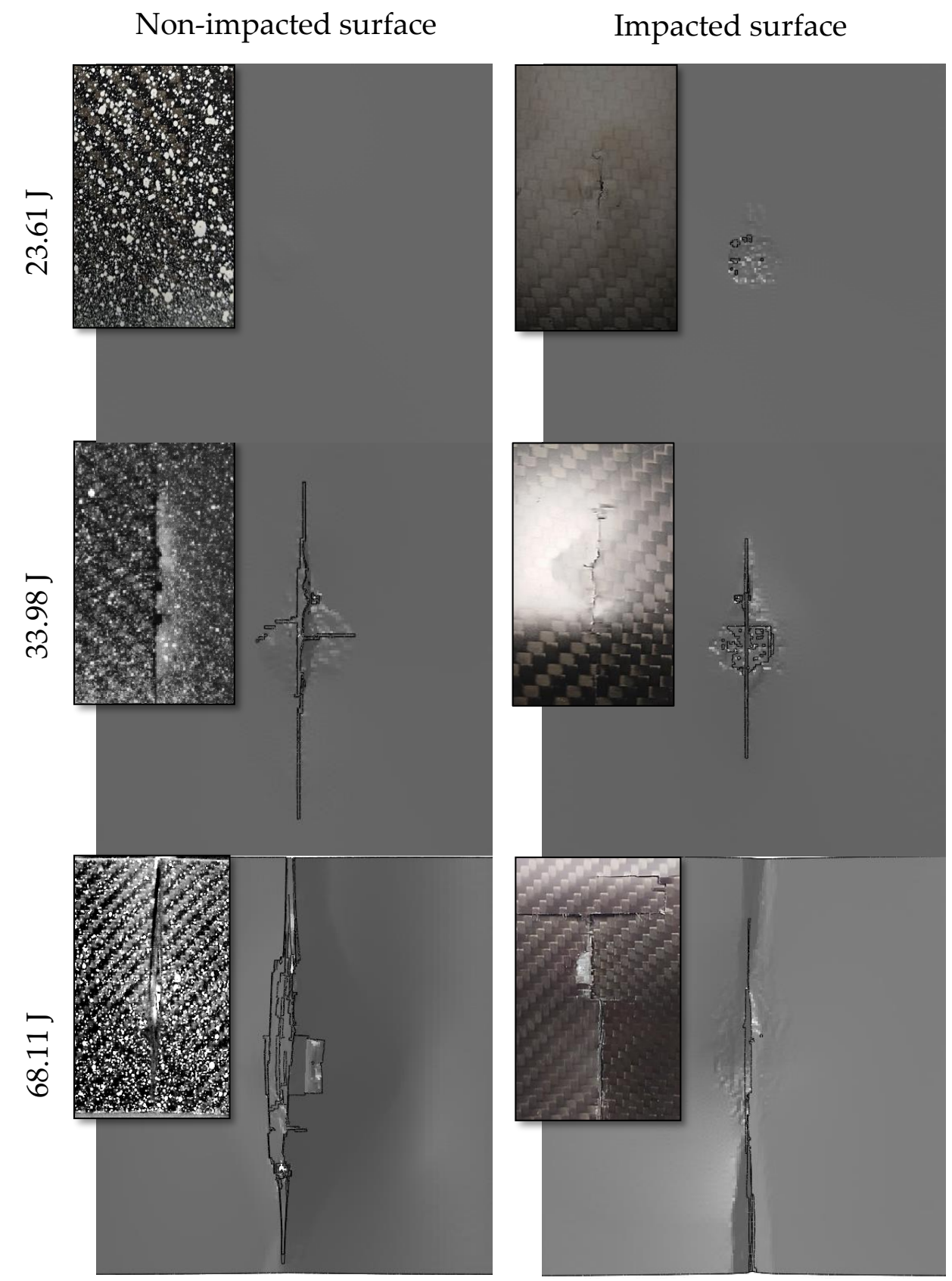

Figure 4.12: Experimental damage at the composite plate at the striking and non-striking surfaces and comparison with numerical simulations at different energy levels. 


\section{Comparison of the strain fields with DIC}

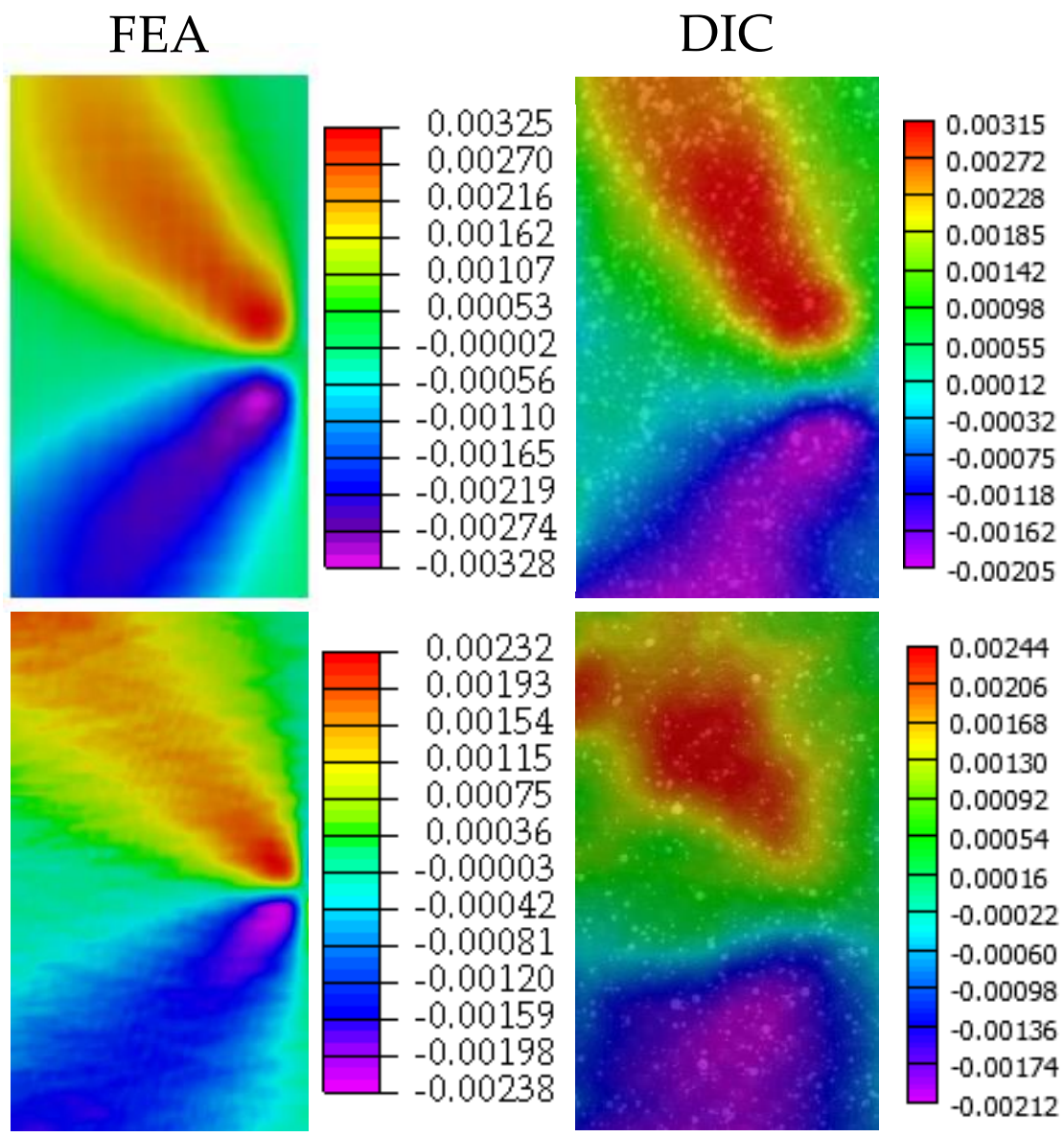

Figure 4.13: Comparison of the strain fields obtained by means of FE simulation and DIC for an impact energy level of $23 \mathrm{~J}$ (top) and $68 \mathrm{~J}$ (top). The variable represented is the shear deformation $\varepsilon_{x y}$. Due to symmetry, only the left half of the plate is represented.

DIC has been implemented to the collection of images recorded by the high speed camera during the charpy impact tests. Because of the changes in lighting that arise when the plate starts to bend in the impact direction, the 
right half of the ROI in the visible surface of the plate suffers from intensity gradients that reduce the accuracy of the method at that region. For this reason, only the left half of the plate will be studied, as there is symmetry in both the loading and boundary conditions along its vertical middle axis.

After damage initiation, surface cracks cause alterations to the speckle pattern and in the failed areas correlation can no longer be achieved. Furthermore, the fact of employing one single camera for the recording and not stereoscopy limits the accuracy of the method as the deflection of the panel cannot be captured and all the strain phenomena are assumed to be bidimensional. These drawbacks can be avoided if focusing only on the first stage of impact, when the damage and deflection of the plate are still low enough to neglect the tridimensionality of the impact. Results shown in Fig. 4.13 do correspond to a time of $0.2 \mathrm{~ms}$ since the initiation of contact between the anvil and the plate.

When comparing the strain fields obtained from this method and the ones achieved through numerical simulation, several similarities in distribution and peak values are found (Fig. 4.13). The top row of Fig. 4.13 illustrates the shear strain fields at the beginning of the impact of $23.61 \mathrm{~J}$ of energy. The peak positive strains present very close values for both methods, whereas peak negative values are lower with DIC. The case represented at the bottom row of Fig. 4.13 corresponds to an impact energy of $68 \mathrm{~J}$. Even though the predicted maximum and minimun shear strain values are similar between FE and DIC, in this case image correlation does not provide an accurate distribution of the field. These discrepancies may be due to the larger deflection expected for impacts with greater input energies, as the tridimensionality issue stated previously will be aggravated.

\subsection{Accuracy of the methodology}

In view of the results presented in the previous section, several conclusions can be drawn. First, the experimental impact tests performed on CFRP plates with a charpy pendulum result in damage patterns and energy absorption values consistent with similar studies on the literature for the different impact energies tested. The experimental study has two main limitations: the tests were not recorded both on the frontal and lateral sides of the set-up (as only one high-speed camera was available) and there is no posterior X-ray or CT 
examination of the damaged plates. The data would have been useful for the verification of the obtained results.

Secondly, an adequate material model for the composite plate requires a proper establishment of damage initiation and evolution criteria. The employed VUMAT subroutive based on the Hashin criteria for fabrics in conjunction with the continuum damage mechanics (CDM) approximation leads to an accurate numerical modeling of the macroscopic damage observable in the plates at the different energy levels. Besides, the employment of a quadratic stress criterion for damage initiation and the energy-based Benzeggagh-Kenane criterion for failure enhances a realistic modeling of the delamination phenomena. In fact, some characteristic features of low velocity impact on CFRP plates such as the delamination cone have been predicted with the numerical model presented in this section.

The digital image correlation (DIC) technique has been applied to measure the strain fields across the non-impacted surface of the plate during loading. The accuracy of this approach decreases as the out-of-plane deflection of the plate increases and therefore the most reliable results are obtained at the beginning of the impact. This limitation could be overcome in the future by employing two high-speed cameras recording the plate from different angles, as the three-dimensional reconstruction of the deformed area would be enhanced. 



\section{Chapter 5}

\section{Numerical modeling of a human head}

\subsection{Head FE models in the literature}

Given the exposed limitations of experimental testing on human heads or surrogates, both analytical and numerical models have been developed since approximately the middle of the twentieth century. The complexity in geometry and material modeling has progressively grown with the increasing capacity of computational systems.

Therefore, pioneering models were restricted by the computational expense and the existing FEM algorithms. For this reason, the most feasible approaches consisted of two-dimensional models comprising just one or two tissues in order to study some specific phenomena. In this Thesis, the focus will be set on three-dimensional human head numerical models and their application to impact environments. Nonetheless, more detailed literature reviews of the evolution of FE head models on different applications can be found in Voo et al. (1996) [186], Raul et al. (2008) [187], Samaka and Tarlochan (2013) [188] and Tse et al. (2014) [189].

One of the first published 3D head FE models was presented by Ward and Thompson (1975) [190], and posteriorly improved. Nahum et al. (1977) [191] employed this model for the validation of their experimental tests on intracranial pressures. Despite being an early model, its level of detail was similar to the current models. 
In 1993, one of the best known head models, developed by the Wayne State University, was presented in Ruan et al. [192,193]. This FE model was divided in seven tissues. Three of them (scalp, falx cerebri and dura mater) were modeled with membrane elements whereas the rest (compact bone tables, diplöe, CSF and brain) employed 8-node solid elements. This prior model was updated by Zhang et al. (2001) [194], and became the Wayne State University Brain Injury Model (WSUBIM). The improved features comprised the differentiation of the fluid-filled structures (sagittal and transversal sinuses and ventricles) and the brain lattice was divided into the gray and white matter, brainstem and cerebellum. Also, the membranes pia mater and tentorium were included and the whole mesh was refined. The final version of the model came in 2004 in Zhang et al. [129], where greater overall element sizes were taken and the material properties of some tissues like the brainstem or white/gray matter accounted for the anisotropic behaviour of the CNS.

In parallel, in 1997, Kang et al. [195] developed the first version of the University Louis Pasteur Finite Element Head Model (ULP FEHM), which was improved in 1999 by Willinger et al. [196]. This second version was built from MR images and was constituted by six tissues: skull bone, falx, tentorium (with shell elements) and subarachnoid space, brain hemispheres and brainstem (using brick elements). The brain is modeled as a viscoelastic material whereas the remaining tissues are considered as linearly elastic. The ULP has been repeatedly improved in order to cope with different head injury mechanisms. For instance, the version presented in 2003 by Willinger and Baumgartner [197] was able to predict skull fracture by establishing a failure criterion based on maximum stresses for the cranial bone layers. In the work by Deack and Willinger in 2009 [198], the model was renamed as Strasbourg University Finite Element Head Model (SUFEHM), and it allowed the prediction of further intracranial injuries such as DAI or SDH. The latest version of the SUFEHM [134] included the anisotropy of the CNS by means of an averaging of the principal axonal directions from DTI images.

Another finite element head model that is still being employed up to date was firstly developed by Kleiven and Hardy (2002) [199]. It included the scalp, skull (differenciating between compact and trabecular bone), brain (separated gray and white matter), ventricles, meninges, CSF and a representative set of parasagittal bridging veins (as truss elements). Several skull-brain interface types were tested against experimental data, achieving the best results with tied nodes between a CSF layer and the adjacent membranes, even though pos- 
terior versions consider sliding interfaces. The hyperelastic material properties of the brain tissue have been updated along time, first from an homogeneous approach, and finally by including white matter anisotropy [200] through the voxelisation of DTI data.

The University College of Dublin also developed a FE head model based on CT images, the UCDBTM. It was firstly published in 2003 by Horgan and Gilchrist [201], with a level of differenciation of ten tissues: scalp, compact bone, diplöe, cerebrum, cerebellum, brainstem, membranes (pia mater, dura mater, falx and tentorium) and CSF. For the latter tissue, hybrid elements from Abaqus were employed to avoid the difficulties of dealing with nearly incompressible materials. Nonetheless, this element type was replaced by solid elements with a sliding interaction with the meninges in the most recent versions of the model [202].

A recently developed FE head model was presented by Tse et al. (2014) [203] and developed from the segmentation of CT images of a middle-aged man. Their model, meshed up with linear tetrahedral elements, included the following tissues: skull, upper neck, teeth, nasal cartilage, scalp/skin, gray/white matter, brainstem, cerebellum, CSF, midbrain and the ventricular system. The CSF layer was model as a solid with fluid-like properties and sliding boundary conditions on its interface with the skull and brain. This head model has then been employed by its developers to evaluate helmet protection against blast threats [204].

\subsection{Model development}

\subsubsection{CT image segmentation}

The base for the development of our numerical model is a set of computed tomography (CT) images from a male anonymous subject provided by Hospital Universitari i Politècnic La Fe (Valencia). The head dimensions of the subject (width, breadth) are 19.7 and $15.8 \mathrm{~cm}$, respectively, which lie within the average measures for the $50^{\text {th }}$ percentile of an adult male [205].

Image segmentation has been carried out by means of the software ScanIP $\mathrm{v} 4.2$ [206], which allows the definition of masks and their further transformation to geometric solids and finally to finite element mesh. Two main considerations have been taken into account during the segmentation process: 
- Topological features should be accurate enough to enhance geometryspecific responses, which is one of the main advantages that provides the segmentation of medical image.

- Tissue differentiation, though preferrable to analyse specific injury mechanisms, involves a large amount of uncertainty in the model and takes a great computational effort. As the purpose of the head model developed in this section is the achievement of a proper material modeling of the different living tissues to match experimental data, the number of variables should be limited to enhance a better comprehension of the behaviour of each tissue.
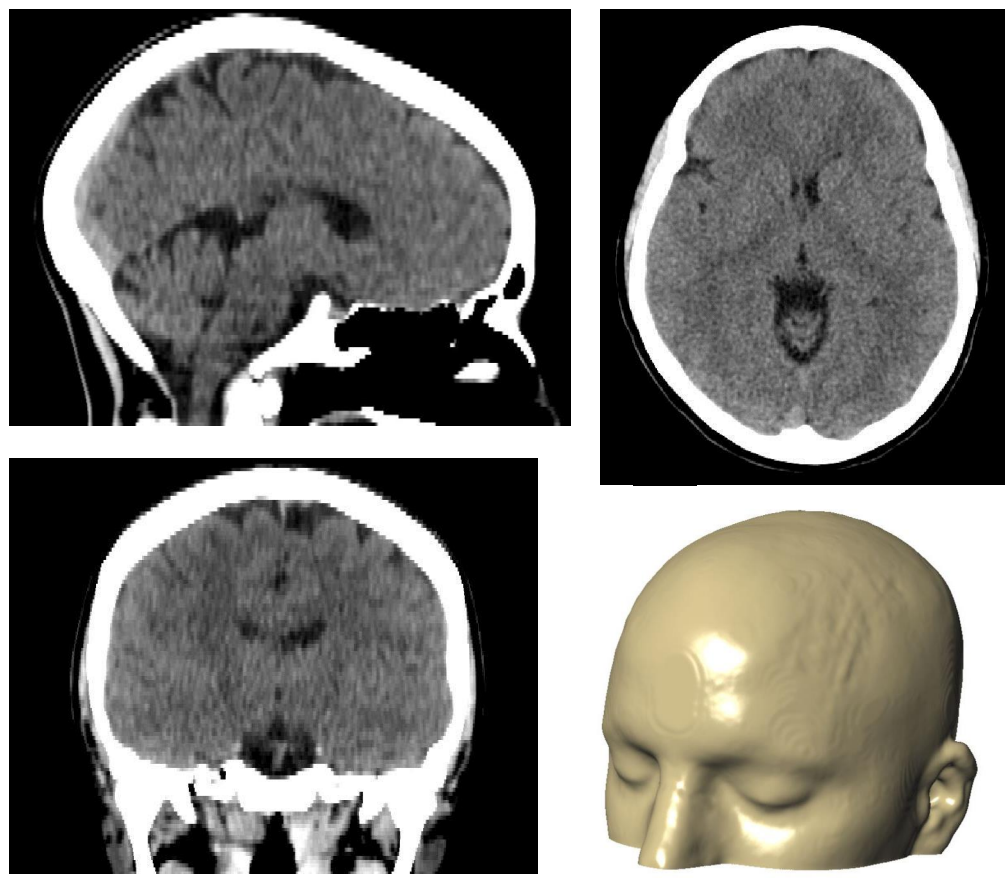

Figure 5.1: CT images of the head of a human subject in different views: sagittal (top left), horizontal (top right) and coronal (bottom left). Threedimensional rendered reconstruction performed with ScanIp (bottom right).

One inherent limitation of the procedure is the resolution of the CT im- 
ages. As opposed to other imaging techniques such as magnetic resonance (MR), which are more costly in both time and budget, computed tomography is a less expensive technique medically useful for rapid diagnostic [207]. Nonetheless, even though this technique allows an adequate distinction between bone, fluid and soft tissues, the latter cannot be easily differenciated from one to another as most of the intracranial contents posess similar densities. As a result, the grayscale representing these tissues is limited and the boundaries between intracranial regions are not perceivable. This fact hinders the automatic segmentation (by, for example, the thresholding technique) of the different brain structures.

Therefore, a manual segmentation of some of the head tissues has been performed. In order to achieve an anatomically coherent model, the dimensions of the manually constructed parts have been based on physical reported values. For instance, as can be seen in Fig. 5.1, the skull bone is clearly differenciated from the soft tissues but the porosity of the trabecular bone conforming the diplöe layer is not captured. As one of the objectives in this study is the assessment of cranial fracture, it is relevant for the numerical head model to have a distinction between the different bone layers as many fracture mechanisms cannot be fully explained by considering the skull as an homogenised tissue. Hence, an internal cranial layer has been segmented based on the geometrical features reported by Boruah et al. (2015) [208] (from a MRI study) and Voie et al. (2014) [209] (combining CT and transcranial focused ultrasound (TcFUS)) in order to ensure a reallistic distribution of the skull thickness between the inner cortical, diplöe, and outer cortical layers.

It is relevant to clarify that this segmentation approach for the skull is based on the macro-scale level under the assumption of an homogeneous tissue for each of the compact bone tables and diplöe. Indeed, the cranial bone is highly heterogeneous along all the hierarchical levels [10]. Compact bone, at the mesoscale, can be regarded as a biphasic heterogeneous material with pores and a mineralised collagen matrix. Increasing the detail level, at the nanoscale, the solid phase reveals an arrangement of spatially oriented collagen fibers, which group in different structural units (osteons) while the pores are traversed by vascular tissue [210]. The cancellous bone is composed by non-homogeneously arranged collagen-based structures (trabeculae) leaving an important volume of voids that can contain red or yellow marrow.

An analogous approach has been taken to model the subarachnoid space. 
The CT images from Fig. 5.1 reveal a certain boundary around the brain tissue in some regions but in most of them it appears to be attached to the neighbouring skull bone. Nonetheless, as described in Chapter 2 of this Thesis, there is a whole complex structure forming the brain-skull interface, constituted by the three meninges (dura mater, arachnoid and pia mater). The dura and arachnoid are adjacent membranes. The latter is separated from the pia mater by the subarachnoid space, which mainly consists of a fluid cavity of CSF with numerous embedded blood vessels connecting the different layers. An explicit model of this natural geometry would be extremely complicated to develop and computationally expensive. For this reason, the space between the skull's inner surface and the brain has been segmented as a single layer with homogenised properties of the involved tissues, in order to ensure a certain stiffness while allowing the relative brain-skull displacement. To simulate the displacement of a fully immersed body, the whole brain surface is assumed to be surrounded by the CSF layer. In accordance with previous numerical models [211], the average thickness of this layer has been set to approximately $2 \mathrm{~mm}$.

As a result of the segmentation process, a total of six masks have been defined, each corresponding to one living tissue: the cranium (divided into its two compact bone layers and the diplöe core), facial bones, CSF, brain and scalp.

\subsubsection{Mesh}

The resulting masks have been automatically meshed using ScanIp. Linear tetrahedra (labelled as C3D4 in Abaqus) have been selected for the meshing, obtaining a total of 379550 elements with an average size of $3 \mathrm{~mm}$. The resultant mesh, with its different material constituents, is illustrated in Fig. 5.2 .

There are several benefits and restrictions regarding the element type chosen. On the one hand, a mesh of fully integrated tetrahedral elements can be automaticaly obtained from ScanIp and it allows to maintain the geometry of the initial masks, since it is specially complex to use manual meshing techniques for the cranial bones. Additionally, this element type does not suffer from hourglass, which becomes a relevant issue when involving impact problems. On the other hand, tetrahedral elements can experience volumetric 
locking when dealing with nearly-incompressible materials, which is the case of many human soft tissues. The issue of volumetric locking has been extensively addressed in the literature [212-216] and several formulations have been proposed to overcome this problem in FEM solid mechanics. Nonetheless, a common and effective technique to avoid this undesired phenomenom is to employ higher order elements with reduced integration [216].

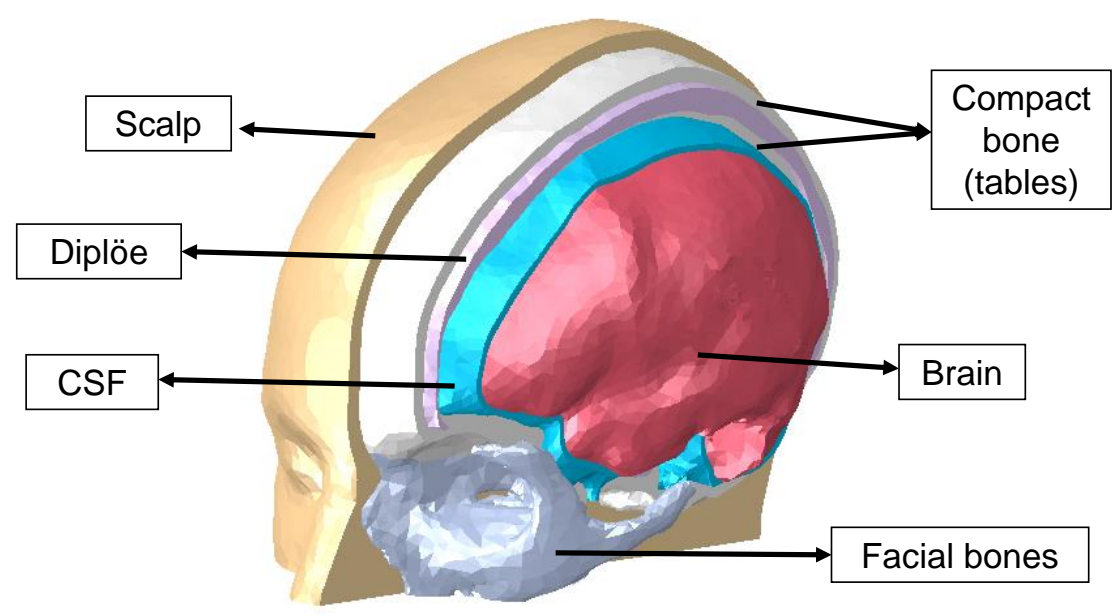

Figure 5.2: Resulting finite element mesh from CT image segmentation. The six principal tissues included in the model are illustrated.

A comparison study between linear and quadratic tetrahedral elements has been performed in order to analyse the criticality of employing linear elements on the accuracy of the results, specifically in soft tissues. This comparison is based on the simulation of Nahum's experiment [191], which is described in detail in Section 5.4.2, with two FE models: one meshed with linear (C3D4) tetrahedral elements and another with quadratic (C3D10) tetrahedra. After the simulation of the impact test in both cases, the pressure distribution along the brain tissue is analysed. It was observed that the the variation in the pressure contours is minimal, whereas simulation time of the quadratic mesh is almost an order of magnitude greater than the simulation time required for the linear mesh. Therefore, the linear tetrahedral mesh that constitutes the baseline model, in the cases to be studied, does not exhibit an accused 
suffering from volumetric locking and will be employed due to its reduced computational cost.

The mesh is continuous, with shared nodes between adjacent masks in order to avoid setting contact interactions or constraints between surfaces and thus save computational expense and calculation errors.

\subsection{Material modeling}

Given the wide dispersion in material constitutive models found in the literature for the different head tissues, either based on experimental or numerical studies, achieving an accurate tissue definition is a complex target. Taking into account the level of tissue differentiation of the head numerical model presented here, mechanical properties of similar FE models from the literaure have been taken as a reference to validate its overall impact response. As a result, five sets of mechanical properties for the head structures have been arbitrarily chosen from the literature. The modeling strategy and material constants for each of them are summarised in Table 5.1 (each set of material models will be denoted henceforth as MP and a number indicating the work it has been extracted from).

A work by Gilchrist and O'Donoghue (2000) [217] is denoted as MP1, in which a human FE model is developed to assess its impact response. Brain is modeled as a linear elastic material without time-dependency. The material properties of the scalp are not specified in the article. Label MP2 is employed for the material properties from the head model by Ruan et al. [192], which introduces additional viscoelastic properties to the brain tissue.

MP3 refers to the properties employed by Kleiven (2006) [218] in the numerical head model developed by the Kungliga Tekniska Högskolan (KTH, Stockholm). From the numerical study on motorcycling accidental reconstruction conducted by Kang et al. (1997) [195] the set of material properties has been taken and denoted as MP4. In this case, brain tissue is considered as a fluid-saturated solid and its constitutive characterisation is based on the properties of water, defining its elastic behaviour in terms of its bulk modulus $K=\frac{E}{3(1-2 \nu)}$. 


\begin{tabular}{|c|c|c|c|c|c|}
\hline & $\begin{array}{r}\text { MP1 } \\
{[217]}\end{array}$ & $\begin{array}{l}\text { MP2 } \\
{[192]}\end{array}$ & $\begin{array}{c}\text { MP3 } \\
{[218]}\end{array}$ & $\begin{array}{l}\text { MP4 } \\
{[195]}\end{array}$ & $\begin{array}{r}\text { MP5 } \\
{[219]}\end{array}$ \\
\hline \multicolumn{6}{|c|}{ Compact bone } \\
\hline$\rho\left(\mathrm{kgm}^{-3}\right)$ & 3000 & 3000 & 2000 & 1800 & 1210 \\
\hline$E(\mathrm{MPa})$ & 5465 & 12200 & 15000 & 15000 & 8000 \\
\hline$\nu$ & 0.22 & 0.22 & 0.22 & 0.21 & 0.22 \\
\hline \multicolumn{6}{|l|}{ Diplöe } \\
\hline$\rho\left(\mathrm{kgm}^{-3}\right)$ & 1750 & 1750 & 1300 & 1500 & 1210 \\
\hline$E(\mathrm{MPa})$ & 2864 & 5660 & 1000 & 4500 & 8000 \\
\hline$\nu$ & 0.22 & 0.22 & 0.22 & 0.22 & 0.22 \\
\hline \multicolumn{6}{|l|}{ Facial bone } \\
\hline$\rho\left(\mathrm{kgm}^{-3}\right)$ & 2100 & 2100 & 2000 & 3000 & 1210 \\
\hline$E(\mathrm{MPa})$ & 5000 & 5000 & 15000 & 5000 & 8000 \\
\hline$\nu$ & 0.23 & 0.23 & 0.22 & 0.21 & 0.22 \\
\hline \multicolumn{6}{|l|}{$\mathrm{CSF}$} \\
\hline$\rho\left(\mathrm{kgm}^{-3}\right)$ & 1040 & 1040 & 1000 & 1040 & 1040 \\
\hline$E(\mathrm{MPa})$ & 0.1845 & 1.49 & 1.26 & 0.012 & 1.314 \\
\hline$\nu$ & 0.499 & 0.489 & 0.4999 & 0.49 & 0.4999 \\
\hline \multicolumn{6}{|l|}{ Brain } \\
\hline$\rho\left(\mathrm{kgm}^{-3}\right)$ & 1040 & 1040 & 1040 & 1040 & 1140 \\
\hline Behaviour & Elastic & Elastic & Hyperelastic* & Elastic & Elastic \\
\hline Constants & $\begin{array}{c}E= \\
0.558 \mathrm{MPa} \\
\nu=0.485\end{array}$ & $\begin{array}{c}E= \\
0.307 \mathrm{MPa} \\
\nu=0.4996\end{array}$ & $\begin{array}{l}\mathrm{C}_{01}=62 \mathrm{~Pa} \\
\mathrm{C}_{10}=69 \mathrm{~Pa}\end{array}$ & $\begin{array}{c}K= \\
1.125 \mathrm{GPa}\end{array}$ & $\nu=0.48$ \\
\hline Viscoelastic & & $\mathrm{g}_{1}=0.682$ & $\begin{array}{l}\mathrm{g}_{1}=0.636 \\
\mathrm{~g}_{2}=0.363\end{array}$ & $\mathrm{~g}_{1}=0.659$ & $\mathrm{~g}_{1}=0.682$ \\
\hline terms $* *$ & - & $\tau_{1}=0.0286$ & $\begin{array}{c}\tau_{1}=0.008 \\
\tau_{2}=0.15\end{array}$ & $\tau_{1}=0.0069$ & $\tau_{1}=0.0286$ \\
\hline \multicolumn{6}{|l|}{ Scalp } \\
\hline$\rho\left(\mathrm{kgm}^{-3}\right)$ & & & 1130 & 1200 & 1130 \\
\hline$E(\mathrm{MPa})$ & - & - & 16.7 & 16.7 & 16.7 \\
\hline$\nu$ & & & 0.42 & 0.42 & 0.42 \\
\hline
\end{tabular}

*Hyperelastic constants correspond to a Mooney-Rivlin material in Abaqus [155] **Viscoelastic constants for a Prony Series in Abaqus [155]

Table 5.1: Summary of the mechanical properties of the different head tissues of the five models extracted from the literature. 
Finally, the last set of mechanical properties has been extracted from the medical image-based head model developed by Tse (2013) [219]. There are several similarities with the head model presented in this chapter as both are developed from the segmentation of medical images and employ the same meshing technique and element type. The model in [219] accounts for additional segmentation, subdividing the cerebrum as gray and white matter, brainstem, cerebellum, etc. The material properties set to each of this central nervous system (CNS) constituents are, however, essentially the same so these could be merged as a single tissue. The different skull layers are also separated in the mesh, but the material properties set to each of them are the same, so the whole calvarium is considered as an homogeneous tissue.

\subsection{Calibration of the model}

As it could be seen in the literature review on skull biomechanics in the second chapter of this Thesis, there is a large dispersion regarding its material characterisation. It is not a surprising result given both the intra-subject and inter-subject differences on the human skull. Even when taking into account a single specific head, its mechanical response can vary depending on the cranial region, the nature of the applied load, the loading rate, the boundary conditions, etc. Therefore, it is nearly impossible to interpolate such variables altogether with others like age or sex to draw a proper general constitutive law of the skull bones.

The head model developed in this chapter contains the geometric features of a specific subject (such as skull thickness and dimensions, curvature, scalp thickness) and it is to be expected that its mechanical behaviour will be influenced by them. Nonetheless, in order to achieve a reasonably realistic response of the developed model, a calibration study has been carried out using some experimental impact tests on post mortem human subjects available in the literature. Specifically, low-velocity blunt impact tests by Yoganandan et al. (1995) [21] and Nahum et al. (1977) [191] have been numerically recreated in Abaqus/Explicit using the in-house developed head model.

The first simulation was based on the set-up employed by Yoganandan et al. [21], which will be more extensively explained in the following subsection. As the experimental work deals with dynamic cranial fracture, the aim of this simulation is to calibrate the failure mechanisms of the numerical head model 
for both the skull bones and the scalp layer. To enhance the modeling of skull fracture, two element-based techniques, implemented in a user-defined subroutine (VUSDFLD in Abaqus/Explicit [184]) have been tested: mechanical properties degradation and element erosion. The first technique consists of setting a reduced elastic modulus to the element whose stress level exceeds a certain threshold value. The 'failed' element acquires a stiffness value $95 \%$ lower than the initial one. One benefit of this technique compared to the direct deletion of the element is that there is no mass loss, but the degraded elements may reach unsustainable deformations when dealing with certain loading modes. Both bony constituents of the skull show a brittle behaviour separately, and for this reason a continuum damage mechanics (CDM) approach has been rejected in this first approximation. The Rankine's criterion will be employed to determine the failure of the element for both techniques. According to this maximum normal stress criterion, failure occurs in a multiaxial state when

$$
\sigma_{1} \geq \sigma_{\mathrm{ut}} \quad \text { or } \quad \sigma_{3} \leq \sigma_{\mathrm{uc}}
$$

being $\sigma_{1}, \sigma_{2}$ and $\sigma_{3}$ the maximum, intermediate and minimum principal stresses, respectively $\left(\sigma_{1} \geq \sigma_{2} \geq \sigma_{3}\right) . \sigma_{\mathrm{ut}}$ and $\sigma_{\mathrm{uc}}$ represent the uniaxial tensile and compressive (negative) strengths of the material, respectively.

Once an adequate constitutive law for the scalp and cranial bones as well as a calibrated response to mechanical failure of each of these tissues are determined, the second experimental test from the literature is simulated. This was conducted by Nahum et al. [191] and consisted of blunt impacts performed on cadaveric heads. Intracranial pressures at different locations of the cerebrum were measured. In the context of this Thesis, the simulation of this experimental test is intended to achieve the most suitable constitutive laws for the brain tissue and the CSF layer.

\subsubsection{Dynamic mechanical properties of the cranium and scalp}

As mentioned in Section 1.2, one of the most relevant experimental studies conducted on intact postmortem human heads is the one conducted by Yoganandan et al. (1995) [21]. Twelve unembalmed specimens were submitted to either quasi-static or dynamic loading. These comprised blunt impact tests delivered by a hydraulic piston topped with a hemispherical anvil at different locations and velocities. For the calibration procedure of the numerical model presented here test $\mathrm{n}^{\mathrm{o}} 7$ from [21] has been selected, as the resultant 
force-displacement curve is given. Additionally, the head dimensions of the postmortem subject resemble the numerical model, thus enhancing a better reproduction of the fracture mechanisms.

In the experiment, the anvil impacted the specimen at a constant vertical velocity of $7.2 \mathrm{~ms}^{-1}$ at the vertex region. The test is recreated in Abaqus/Explicit by modeling a rigid hemispherical impactor with the diameter reported in [21] on which a velocity of $7.2 \mathrm{~ms}^{-1}$ is prescribed. The head model is placed in such a way that the impact takes place in the most prominent region of the calvarium, and the vertical displacement of the base of the head is restrained. The replication set-up is illustrated in Fig. 5.3. To model the interaction between the rigid anvil and the head, a surface-to-surface contact algorithm has been chosen.

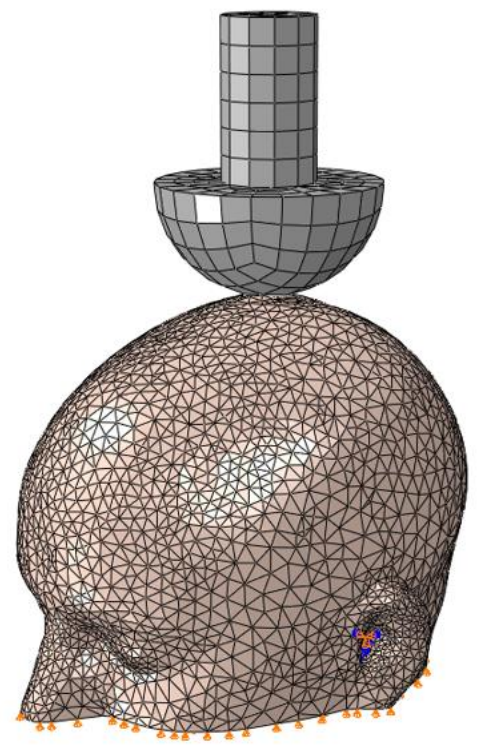

Figure 5.3: Reconstruction in Abaqus/Explicit of the experimental impact test by Yoganandan et al. [21].

\section{Scalp}

The first tissue whose mechanical behaviour will be addressed is the scalp. Skin, as a soft tissue, presents a behaviour such that can be captured by means 
of a hyperviscoelastic constitutive law. This is normally the approach taken in the literature for numerical modeling of surgical interventions. Nonetheless, even though the most external layer of the scalp is skin, there are some differences between them, as discussed in Section 2.3. Mainly, the scalp is the thickest protective layer in the body and it contains a higher volume of blood vessels. Anatomically, it is constituted by five layers of different tissues. Mechanical properties of the scalp as a whole have scarcely been studied in the literature. The tests by Melvin et al. [87] show a typical force-displacement curve, with an initial toe followed by a linear relation and then a non-linear part. The elastic modulus derived from this linear region (until strain levels of $0.3-0.4$ ) is about $16.7 \mathrm{MPa}$. However, there is no information regarding the strength of the material. A recent experimental study by Falland-Cheung et al. [89] reports an average tensile elastic modulus of $22.74 \mathrm{MPa}$ and tensile strengths in the range of $2.75-3.61 \mathrm{MPa}$ depending of the head region. Nonetheless, other recent studies (Pittar et al. ([90]) point to the hypothesis that in impact simulation the hardness of the scalp is the main relevant parameter rather than its tensile properties.

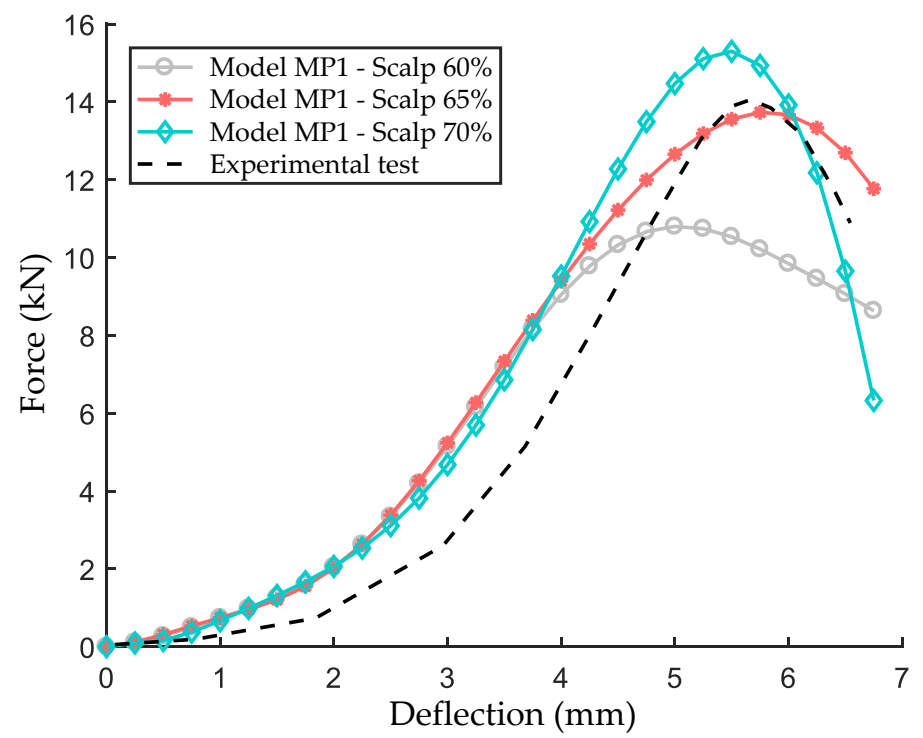

Figure 5.4: Calibration on the ultimate strain for the scalp tissue based on the experiment by Yoganandan et al. [21]. 
The Abaqus/Explicit simulation of the impact test by Yoganandan et al. [21] aims to stablish a proper ultimate stain value for the scalp tissue under dynamic loading for the developed head model. To this purpose, a parametric study has been performed by implementing different $\varepsilon_{\mathrm{u}}$ values in a material user subroutine controling element deletion. The closest results to the experimental response, gathered in Fig. 5.4, were obtained for limit strain values of in the range of $60 \%-70 \%\left(\varepsilon_{u}=0.6-0.7\right)$. Lower ultimate strains led to an unrealistic laceration of the scalp layer. The force-displacement curves illustrated in Fig. 5.4 have been obtained with the set of mechanical properties belonging to MP1, but the same trend was obtained with the remaining four MPs and therefore the selected criterion applies for them all. It can be observed that setting a $\varepsilon_{\mathrm{u}}$ of 0.6 underestimates the peak force by a $40 \%$ and anticipates the failure of the skull. Increasing the threshold strain by a $5 \%$ the predicted peak force is more accurate, but the damage evolution of the head as a whole infers a ductile behaviour which does not resemble the experimental response. Taking into account this considerations, the best fitting is achieved when $\varepsilon_{\mathrm{u}}=$ 0.7 , and this limit strain value will be employed for further calculations.

\section{Cranial bone}

As it was seen in Section 2.1 of this Thesis, some experimental studies addressed the fracture behaviour of the cranial bone by uniaxial or three point bending tests on skull samples. Based on the results presented in some of these, especially the ones by McElhaney et al. [3], Hubbard [5] and Wood [6], some studies in the literature have implemented their fracture threshold values for the construction of finite element head models. The failure criteria adopted in four of these numerical studies, summarised in Table 5.2, have been implemented to the model developed in this section under the impact conditions of Yoganandan et al. [21].

A reduced number of experimental studies have reported the mechanical properties of the cranial layers (tables and diplöe) separately and most of them are referred to the entire through-thickness of the skull. Both cases have been implemented in this study. The failure criteria employed by Raul et al. [220] and Sahoo et al. [148] consider different ultimate stresses for compact and trabecular bone, and also for either tensile or compressive loading. Silver [221], on the other hand, assumed homogeneity at the skull level but distinguished between tensile and compressive strengths. Finally, Evans and Lissner 
[1] provided a combined approach, as their values for compact bone are in fact achieved with the uniaxial loading of three-layered samples, but under the assumption that the diplöe core does not contribute to strengthen the assembly in the longitudinal direction. However, they did provide a compressive strength value of the diplöe tissue isolatedly tested.

\begin{tabular}{lcccc}
\hline & \multicolumn{2}{c}{ Compact bone } & \multicolumn{2}{c}{ Diplöe } \\
& $\sigma_{\mathrm{uc}}(\mathrm{MPa})$ & $\sigma_{\mathrm{ut}}(\mathrm{MPa})$ & $\sigma_{\mathrm{uc}}(\mathrm{MPa})$ & $\sigma_{\mathrm{ut}}(\mathrm{MPa})$ \\
\hline Raul et al. [220] & -145 & 90 & -28 & 35 \\
\hline Sahoo et al. [148] & -132 & 90 & -24.8 & 34.8 \\
\hline Evans and Lissner [1] & $-152.2,-167.4$ & 70.5 & -25.1 & - \\
\hline Silver [221] & -85 & 42.5 & -85 & 42.5 \\
\hline
\end{tabular}

Table 5.2: Ultimate strength values for the cranial bone layers reported in different works from the literature.

$$
\begin{array}{|l|}
\hline \square-\text { Sahoo et al. (2016) } \\
-\smile \text { Raul et al. (2006) } \\
- \text { Silver (1994) } \\
\square-\text { Evans and Lissner (1954) } \\
--\quad \text { Experimental test } \\
\hline
\end{array}
$$
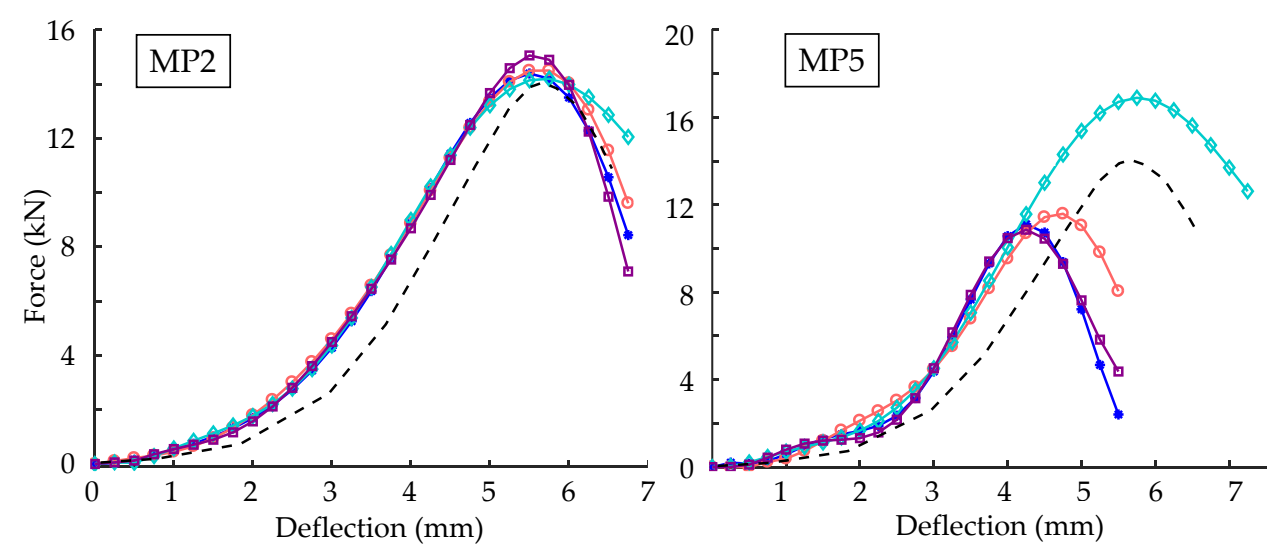

Figure 5.5: Force-displacement response of the in-house numerical head model with different failure criteria for the cranial bone layers. 
Each of the sets of material properties from Table 5.1 will be simulated on the baseline FE head model in conjunction with each of the failure criteria summarised in Table 5.2. As a result, twenty simulations of the impact test from [21] are obtained in order to select the combination of elastic response plus failure criterion for each cranial tissue that resembles most the experimental curve. Simulations dealing with MP1, MP2, MP3 and MP4 reveal consistent results which follow the same trend regarding each failure criteria. In Fig. 5.5 (left) the obtained force-deflection curves for model MP2 have been plotted, even though each of the aforementioned MPs could have been employed as an example. The trend is the following:

- Applying the criteria from Silver [221], the predicted peak force on the head is quite accurate whereas the initiation and evolution of failure is not realistic. Provided that MP1 to MP4 consider cancellous bone as a more compliant material than compact bone and that Silver [221] assumes the same ultimate stresses for both tissues, the strain at failure in the diplöe is from 2 to 15 times greater than the one for the cortical tables. Therefore, fracture propagation under bending loading starts at the inner compact bone layer but does not instantaneously propagate through the porous core but through the outer bone layer, with the diplöe being the last tissue to break. At the force-deflection response level, it can be observed in Fig. 5.5 (b) that the overall force the structure can withstand before its collapse is $4 \mathrm{kN}$ higher than the reported experimentally. This behaviour is not consistent with the brittle behaviour of the cranium.

- In the case of employing the ultimate stress values from Evans and Lissner [1], the resultant peak force is about a $10 \%$ greater than the experiments. This increase is due to the high ultimate compressive stress value assumed for compact bone. However, for this same tissue the $\sigma_{\text {ut }}$ is lower than that employed by Raul et al. [220] and Sahoo et al. [148] and therefore the allowable deformation between damage initiation and collapse is reduced and the skull response appears to be excessively brittle.

- The two remaining approaches, whose limit values (strengths) are very close, lead to similar force-deflection responses, being the criteria from Sahoo et al. [148] the one providing the best aproximation in terms of peak force. The skull's response is slightly more ductile than the experimental with the threshold values by Raul et al. [220] while being 
slightly more fragile with the ones by Sahoo et al. [148]. In order to be conservative, the criteria proposed in the latter work will be employed from now on to characterise the fracture behaviour of the skull bones for the material properties sets MP1 to MP4.

This tendency is not observable in the case of MP5, as illustrated in Fig. 5.5 (right). This finding is coherent as MP5 assumes the skull as a homogeneous entity in terms of mechanical properties. Consequently, when setting low ultimate stresses to the stiffened cancellous bone, which is the case of all the taken criteria except for Silver [221], the whole cranial vault will break prematurely. Even though resulting in the overprediction of the peak head force, the homogeneous approach from Silver is intended for an homogeneous skull model condition. For this reason, for further simulations the material strengths from Silver will be chosen to characterise the fracture response of model MP5.

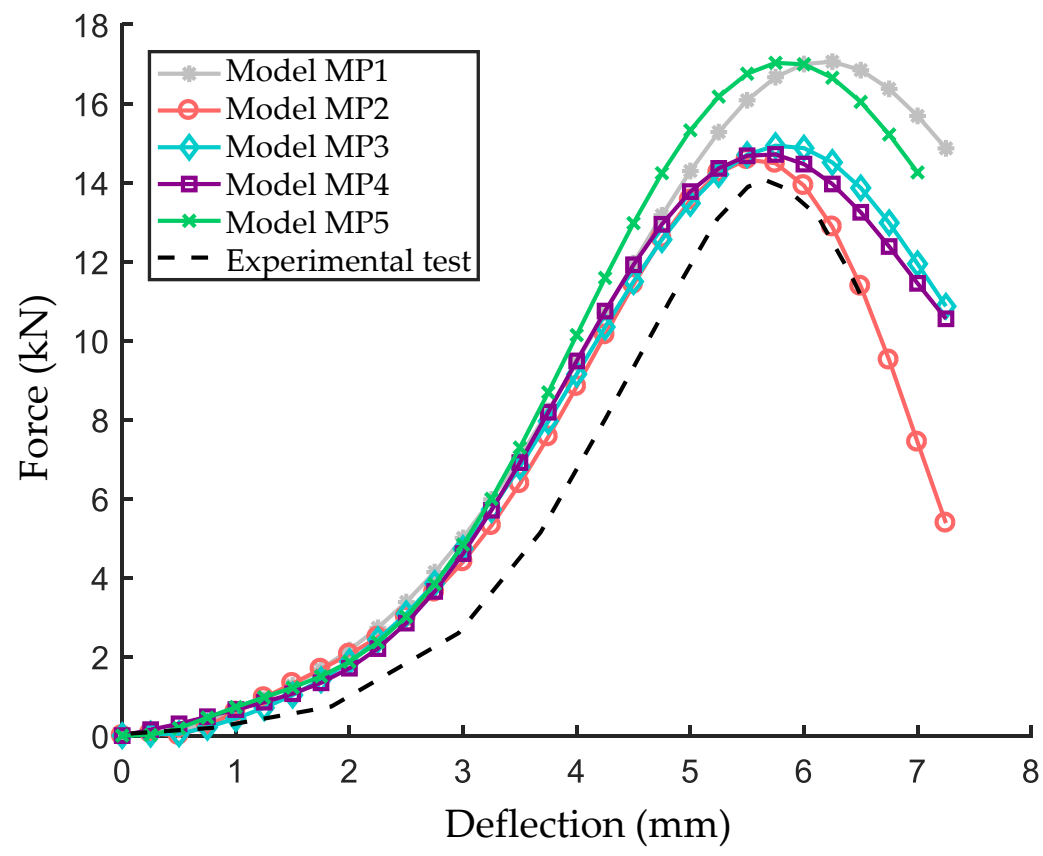

Figure 5.6: Force-deflection response of the head model with each set of mechanical properties against the experimental data by Yoganandan et al. [21]. 
After establishing a certain fracture criterion for the skull bones of each of the MPs chosen and a global one for the scalp tissue, one simulation of the Yoganandan experiment has been again carried out with each resultant MP model. The force-deflection curves obtained for each simulation are depicted in Fig. 5.6. It is noticeable that all curves present the same shape:

- First, a toe region in which the impact force is being attenuated by the scalp layer. As the scalp thickness and material modeling is the same for all the simulated cases, the force-deflection response is identical in this initial segment. A clarification must be made at this point. The scalp thickness from the subject employed at experiment $n^{\circ} 7$ from Yoganandan et al. [21] is $2 \mathrm{~mm}$ greater than the one in the baseline numerical model developed in this section from another specific subject. For this reason, the force attenuation achieved with the model from this work is lower than the achieved in [21]. Numerically, the effect of increasing scalp thickness has been studied employing the baseline geometry from the in-house model, obtaining that greater thicknesses tend to shift the force-deflection curves towards the right of the graph, therefore delaying failure initiation. This finding is in agreement with the experimental work by Delye et al. (2007) [136], in which a positive correlation between scalp thickness at the impact site and maximum displacement is found.

- Second, a linear step in which the cranial bones are sustaining the impact force. From this segment, the overall stiffness of the skull will be posteriorly computed.

- Finally, a damage and failure region determined by the ultimate stresses of the cranial layers. This region is where the greatest discrepancies between material models arise.

By observing Fig. 5.6, the force-deflection response corresponding to two material parameters sets clearly stand out from the average. These correspond to MP1 and MP5. The reason for their disagreement with the rest of the models is the scarce difference in stiffness between the compact and trabecular bone layers of the cranium. In MP5, as it was discussed previously when dealing with fracture thresholds, both bony layers (compact bone and diplöe) own the same elastic properties and this results in an overall skull stiffness higher than the rest. The case MP1 does not consider equal properties between compact bone and diplöe but the Young's modulus of the second is only one 
half of the first. In terms of skull stiffness, the slope of the force-deflection curve falls within the range of the majority, but the peak porce and deflection sustained by the model are a 28.5 and a $15.3 \%$ greater than the experimental, respectively.

The remaining three MPs result in a failure response closer to the experimental. Peak impact forces from MP2 and MP4 are very similar in magnitude (they only differ $0.05 \mathrm{kN}$ between them and $1 \mathrm{kN}$ with respect to the experimental value). Nonetheless, failure initiates earlier in MP2 due to the low ultimate strain value of the diplöe core.

Skull stiffness is one of the experimental values provided by Yoganandan et al. [21] for each of their impact tests and thus the stiffness values derived from the force-deflection curves of each MP have been calculated. All the values are summarised in Table 5.3. Note that the experimental stiffness value presented in Table 5.3 corresponds to the specific case of test $\mathrm{n}^{0} 7$, but the range provided in [21] from all their impact tests is between 2462 and 5867 $\mathrm{N} / \mathrm{mm}$. This means that all the tested MPs would fall within the experimental validity range. Nonetheless, as the antrophometric features from the subject in test $\mathrm{n}^{0} 7$ are similar to the ones of the baseline numerical head model, its specific stiffness value will be taken as a reference to select the optimal material modeling for the cranial bones.

\begin{tabular}{c|cccccc}
\hline & MP1 & MP2 & MP3 & MP4 & MP5 & Experimental \\
\hline Stiffness $(\mathrm{N} / \mathrm{mm})$ & 4938.8 & 5065.3 & 4671.5 & 4932.7 & 5655.9 & 4798.0 \\
\hline
\end{tabular}

Table 5.3: Computed skull stiffness values from each simulation and the experimental value measured by Yoganandan et al.[21]

Both MP3 and MP4 present stiffness values and failure responses close to the experimental. Nonetheless, peak force and the deflection at the peak force are better predicted by MP4 and hence this set of properties will be employed for the following subsection, in which the constitutive behaviour of the intracranial contents will be evaluated. As a brief summary, Table 5.4 presents the constitutive models that will be assumed for the skull bones and the scalp layer, which appear to be the most suitable given the specific geometric features of the numerical model under development. 


\begin{tabular}{l|ccccc}
\hline & Tables & Diplöe & Face & Scalp & CSF \\
\hline$\rho\left(\mathrm{kgm}^{-3}\right)$ & 1800 & 1500 & 3000 & 1130 & 1000 \\
$E(\mathrm{MPa})$ & 15000 & 4500 & 15000 & 16.7 & 1.26 \\
$\nu$ & 0.21 & 0.22 & 0.21 & 0.42 & 0.4999 \\
$\sigma_{\mathrm{u}, \mathrm{c}}(\mathrm{MPa})$ & -132 & -24.8 & - & - & - \\
$\sigma_{\mathrm{u}, \mathrm{t}}(\mathrm{MPa})$ & 90 & 34.8 & - & - & - \\
\hline
\end{tabular}

Table 5.4: Mechanical properties chosen for the scalp and cranial bones of the numerical head model.

\subsubsection{Brain tissue calibration}

After deciding an adequate set of mechanical properties to simulate the elastic behaviour and breakage of the skull bones and the scalp, further experimental data is required in order to address the impact response of the intracranial contents.

To fulfill this requirement, another experimental test on cadaveric human specimens, which has been extensively employed in the validation of FE head models, has been extracted from the literature. The study, conducted by Nahum et al. [191] consisted on blunt impacts performed by a hydraulic piston on the head of the PMHS, which remained seated during the test. The blow was delivered in the frontal head region, in the intersection between the midsagittal plane and a plane with $45^{\circ}$-offset with respect to the Frankfort. Impact force was monitored through a load cell while an accelerometer attached to the centroid of the temporal lobe captured the linear acceleration of the head. Additionally, pressure transducers were inserted through the cranial bones in order to measure the pressure evolution at different locations of the brain tissue, as illustrated in Fig. 5.7.

Experiment 37 from Series I has been taken as a reference and will be recreated in Abaqus/Explicit with the developed head model. The impact scenario in FE is illustrated in Fig. 5.8. The head of the hydraulic piston has been modeled as a stiff material with a foam pad in the striking edge. This padding is employed to damp the force delivered by the impactor and increase the pulse width of the load. The head model is allowed to move freely in the sagittal plane but lateral displacements are constrained, as stated in [191]. 

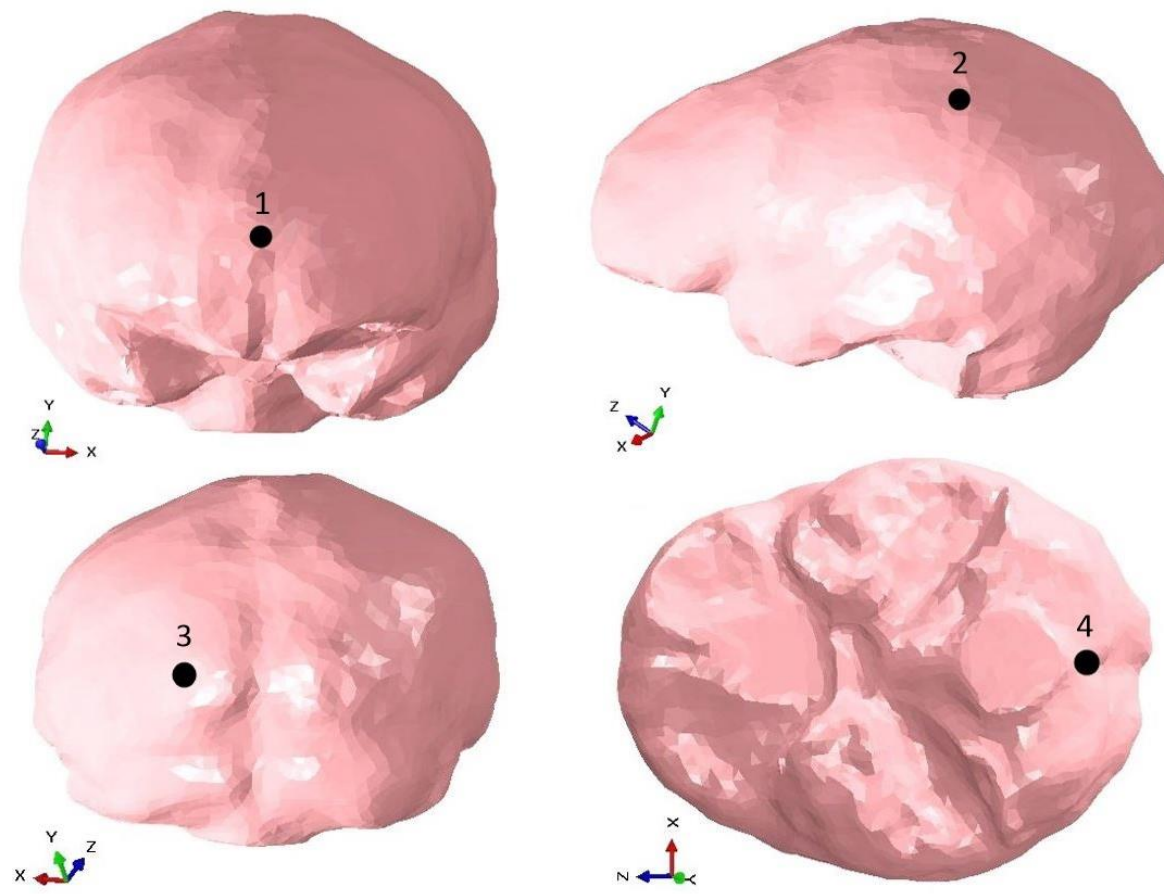

Figure 5.7: Pressure transducers location in the experiment by Nahum et al. [191]: frontal (1), parietal (2), occipital (3) and posterior fossa(4).

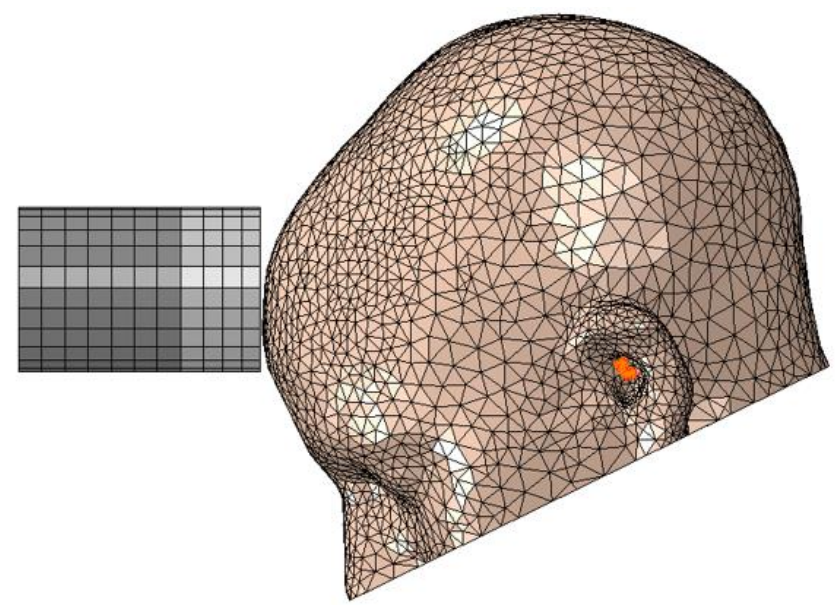

Figure 5.8: Abaqus/Explicit setup of experiment 37 by Nahum et al. [191]. 
Even though the paper by Nahum et al. [191] reports a constant linear velocity of the piston of $9.94 \mathrm{~ms}^{-1}$ during impact, the reported force-time curve cannot be achieved by simply setting this boundary condition. Instead, the stiffness of the padding covering the piston has been adjusted in order to achieve the pulse span from Nahum et al. (depicted in Fig. 5.9 (left)). Once the simulated impact force matches the experimental curve, the linear acceleration at the centroid of the temporal bone is measured and compared to the data given in [191]. As can be appreciated from Fig. 5.9 (right), both curves are properly correlated and the simulation achieves quite accurate peak acceleration values. Therefore it can be concluded that the test conditions of the experiments have been well reproduced in the FE model and properly set and hence the discrepancies in results in terms of intracranial pressures do not arise from the impact setup but from the material modeling itself.
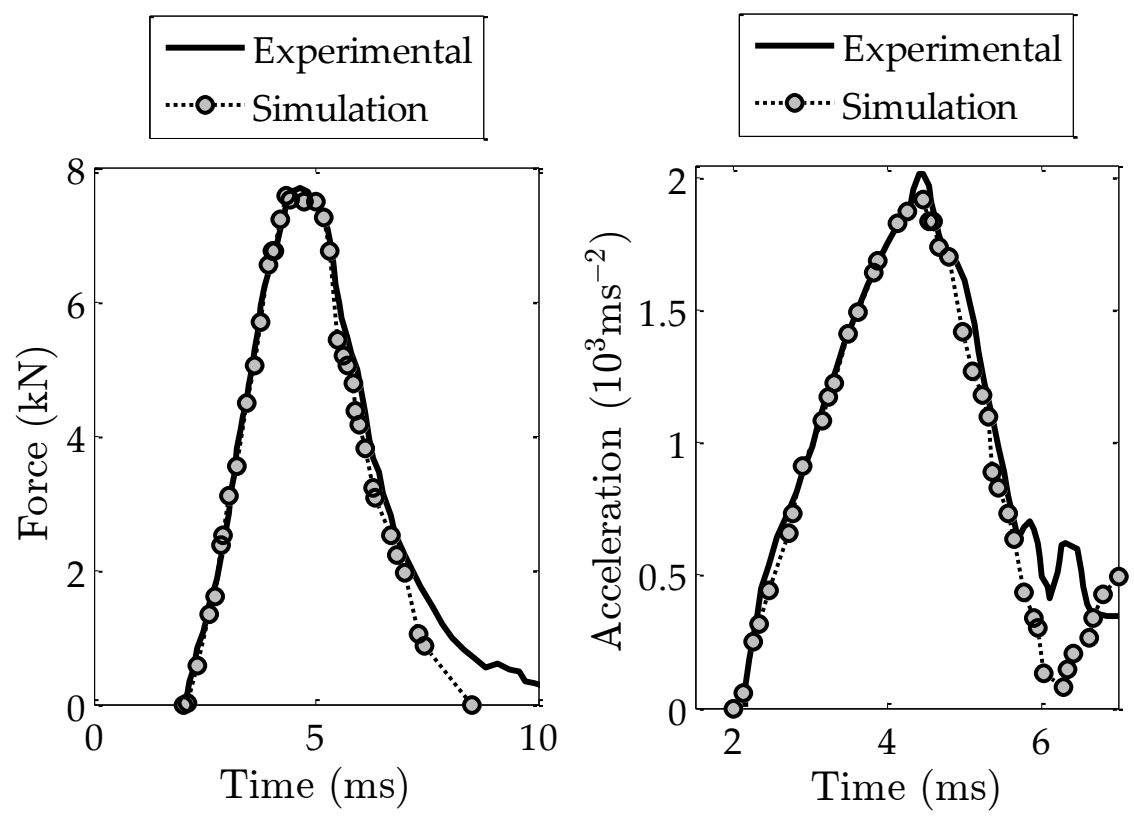

Figure 5.9: Comparison of the force and acceleration histories between the numerical simulation and the experimental curves reported by Nahum et al. [191].

Having defined the mechanical properties of the bones and the scalp in 
the previous subsection, the constitutive models for both the brain and the CSF layers from each of the MP sets summarised in Table 5.1 will be assessed through the simulation of the experiment by Nahum et al. [191]. The pressure histories of the brain tissue measured at the locations defined in Fig. 5.7 will be compared to the experimental curves reported in [191]. The results are represented in Fig. 5.10. Despite the differences in amplitudes, the pressure-time curves corresponding to all MPs follow the same tendency than the recorded experimentally. Namely, positive ICP values (coup) arise in the frontal brain lobe, while negative (contrecoup) pressures appear at the occiput as a result of the wave reflection. Therefore, a pressure gradient is obtained along the brain tissue, having its maximal values at the impact site.

As a reminder, we mention here that MP1 assumed a pure linear elastic behaviour of the brain tissue, while MP2, MP4 and MP5 added a viscoelastic term to the constitutive law. Finally, brain tissue in MP3 was based on an hyper-viscoelastic Mooney-Rivlin material.

Ruan et al. (1994) [193] studied the response of the brain tissue to impact loading based on their human head numerical model. Their work comprised a parametric study varying the bulk modulus $K$ of the cerebrum. One of their findings was the dependency of ICP on the compressibility parameter, obtaining a global shift towards positive pressure values when increasing $K$. This tendency is observable in the results obtained with the head model presented in this section. From the five sets of properties of Table 5.1, MP5 sets the most compressible behaviour of the brain (assuming $\nu=0.48$ ). The resulting simulated intracranial pressures for MP5, depicted in Fig. 5.10 with a green dashed line, tend to underestimate the positive pressure values (at the frontal and parietal locations) while overestimating the magnitude of the negative pressures (in the occipital region and posterior fossa). The same pattern is distinguished for the case MP1 (where $\nu=0.485$ ), but this slight decrease in compressibility reduces the maximum error in ICP estimation from a $86 \%$ (with MP5) to a 7.8\%. As it was discussed in Chapter 2 of this Thesis regarding head injury criteria, intracranial pressure is considered a reliable injury correlator and therefore it is important to achieve an accurate brain response if the purpose of the numerical model is the prediction of head trauma. 

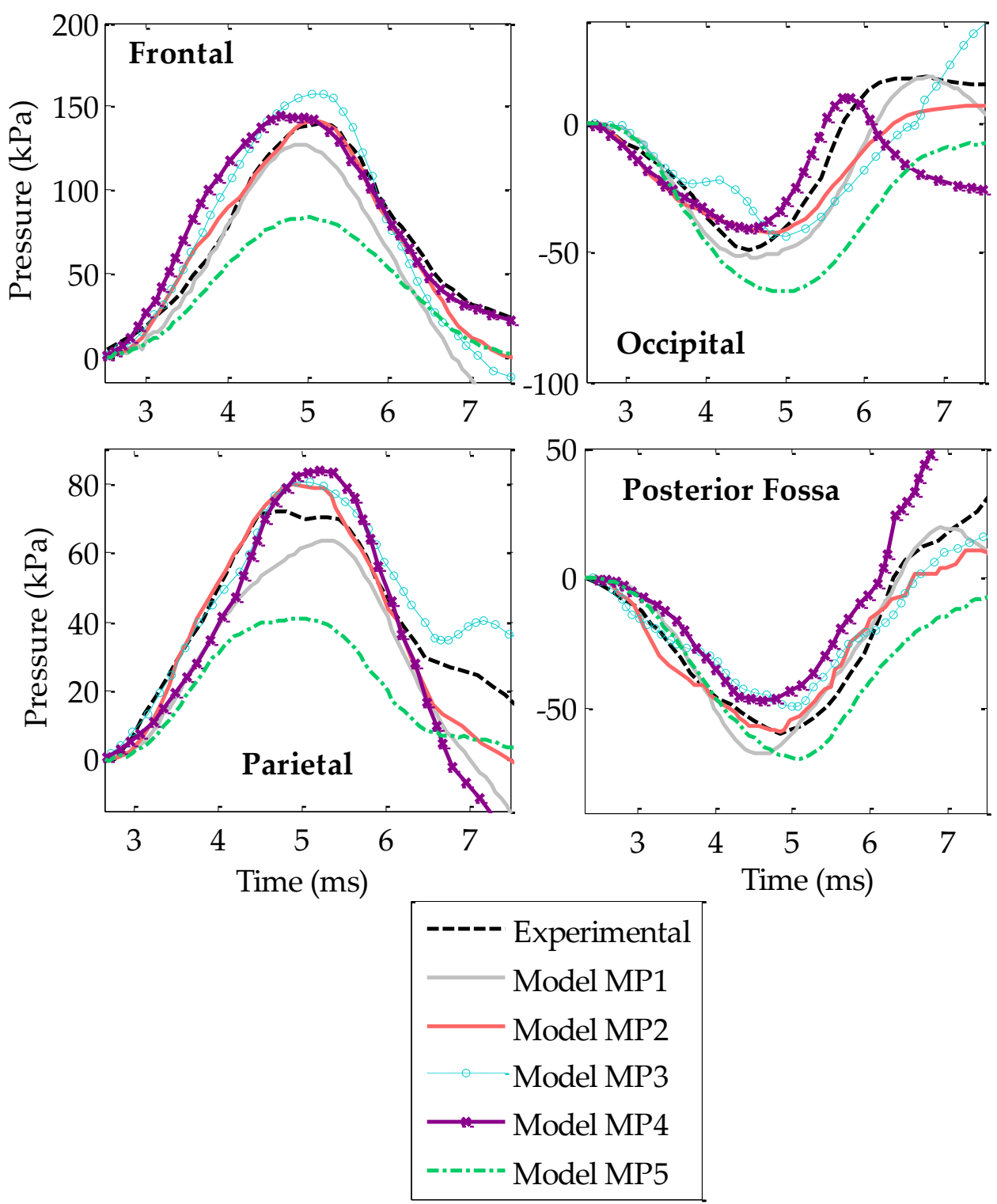

Figure 5.10: Intracranial pressures at different brain locations obtained with numerical simulations and the experimental curves from Nahum et al. [191].

The three remaining MPs involve higher values for the bulk modulus, and 
hence the peak positive values exceed the reported by Nahum et al. [191] and the maximum contrecoup pressures are underestimated. Case MP2, which consists of a non-linear viscoelastic law with a high bulk modulus, presents a good matching with the experimental curves, especially at the frontal and posterior fossa sites. Nonetheless, even though the ICP estimation is reasonably accurate with this simplified material model, most of the latest experimental works on brain tissue have demonstrated that the best characterisation of its constitutive behaviour is through hyper-viscoelastic laws [28, 36, 68, 70]. This feature becomes especially important when dealing with large strain values and strain rates, which is precisely the case of impact loading.
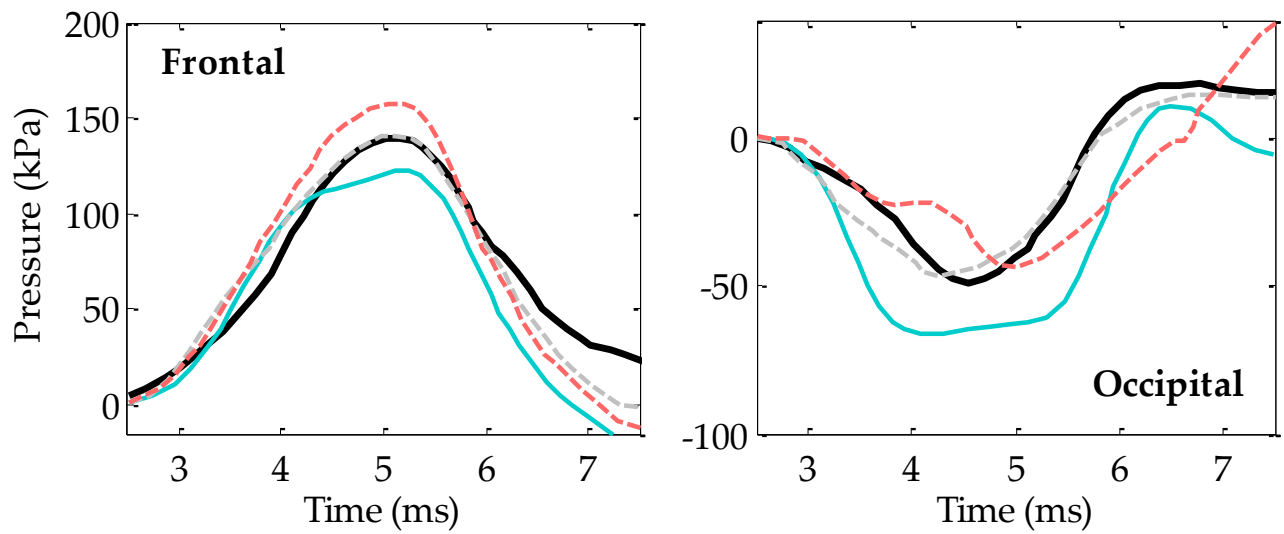

Experimental

$\mathrm{K}=0.021 \mathrm{GPa}$

$\mathrm{K}=0.21 \mathrm{GPa}$

$\mathrm{K}=2.1 \mathrm{GPa}$

Figure 5.11: Effect on intracranial pressure of the variation of the bulk modulus implemented in MP3 under the impact conditions [191].

For this reason, the constitutive model of brain tissue from MP3 is preferred for the numerical head model here presented. As the pressure histories derived from the simulation of the experiment in [191] (represented in Fig. 5.10 with a blue line with markers) present average errors greater than the $20 \%$, a further refinement of the model parameters is necessary to achieve a more accurate response. The initial bulk modulus was set at $2.1 \mathrm{GPa}$ in analogy with water, and it has been decreased by one $(0.21 \mathrm{GPa})$ and two $(0.021$ $\mathrm{GPa}$ ) orders of magnitude. Results from this parametric study are presented 
in Fig. 5.11. It can be seen that setting a compressibility modulus one order of magnitude lower than the one for the water gives the most accurate results (dashed grey lines). This finding is consistent with the statement by Gilchrist and O'Donoghue [217], who suggested that brain tissue may be physically defined more like a gelatin rather than purely water.

This feature comes to be also interesting in terms of computational expense, as the minimum time increment decreases when reaching the incompressibility limit. In Abaqus/Explicit, Mooney-Rivlin hyperelastic materials are defined by the constants $C_{i j}$ and also by the parameter $D_{1}$, whose expression is $2 / K$, being $K$ the bulk modulus of the material. Therefore, the higher the value of $K$, the lower $D_{1}$, and double precision is required for the software in both the preprocessor and the processor.

After determining a proper constitutive model for the brain in the $\mathrm{FE}$ model developed here (whose mechanical parameters are summarised in Table 5.5), the six material constituents considered for the definition of the mechanical behaviour of the human head under impact conditions are characterised. The result is a model validated against low-velocity experimental tests from the literature which can predict intracranial pressure gradients, skull fracture and scalp laceration.

\begin{tabular}{lll}
\hline Properties & $\begin{array}{l}\text { Hyperelastic con- } \\
\text { stants }\end{array}$ & $\begin{array}{l}\text { Viscoelastic } \\
\text { constants }\end{array}$ \\
\hline$\rho=1040\left(\mathrm{kgm}^{-3}\right)$ & $\mathrm{C}_{01}=62 \mathrm{~Pa}$ & $\mathrm{~g}_{1}=0.636$, \\
& & $\mathrm{g}_{2}=0.363$ \\
& $\tau_{1}=0.008$, \\
& $\mathrm{C}_{10}=69 \mathrm{~Pa}$ & $\tau_{2}=0.15$ \\
\hline
\end{tabular}

Table 5.5: Hyper-viscoelastic properties of brain tissue.

\subsection{Validation against high velocity impact condi- tions}

One of the purposes of the head numerical model developed along this chapter is to be able to reproduce a wide range of impact conditions. Specifically, it is intended to be employed in the evaluation of personal protections submitted 
to ballistic threats, which involves loading rates greater than $100 \mathrm{~ms}^{-1}$ as opposed to the presented low/medium velocity tests (Yoganandan et al. [21], Nahum et al. [191]) that lie one order of magnitude less.

For this reason, an experimental study from the literature, performed by Sarron et al. (2004) [222] is recreated in Abaqus/Explicit employing the head model developed in this thesis. The experiments conducted in [222] consist of ballistic impacts with $9 \mathrm{~mm}$ caliber full metal jacket (FMJ) bullets on both dry human skulls and cadaveric heads. The target specimens were protected by a protective plate, whose material varied between tests, which was placed between the projectile and the subject at a certain distance. In all cases the impact site was the parietal region of the head. The analysis performed in this section will account for three of the experiments from [222] using cadaveric whole heads. These tests have been selected for numerical simulations for various reasons: different protective materials are employed (aluminum and aramid), the plates are placed with different separation values (0 or $8 \mathrm{~mm}$ ) and the injury outcome in the head tissues also varies between tests. Therefore, being able to capture the dependency of the numerical head model upon these variables would suggest its adequate application for further high-speed environments.

The set-up conditions for each of these simulated experiments are sumarised in Table 5.6 and Fig. 5.12 illustrates both the initial configurations before (left column) and after (right column) the impact. The first test (\#27) employs aluminun as the protective material, with a plate thickness of $12.5 \mathrm{~mm}$ whereas the other two (\#68 and \#72) use $9.7 \mathrm{~mm}$-thick aramid plates. The plate diameter is the same in all cases $(250 \mathrm{~mm})$ and in the first and second experiments no separation is left between the plate and the specimen's head (stand-off distance $=0 \mathrm{~mm}$ ) whereas in the third case a distance of $8 \mathrm{~mm}$ is set.

The influence of material type and stand-off distance on head injury has been numerically corroborated and the qualitative results obtained in this fashion are in agreement with the findings by Sarron et al. The aluminum plate suffers less deformation under the bullet impact with respect to the aramid one at a zero-stand-off distance level. Even if the thickness is just slightly higher in the metal case, a substantial difference in the observable injuries is found between these two cases. The same situation is drawn when comparing the results between the two aramid-plate cases with different separations with 
respect to the head.

In the aluminum-plate simulation (\#27), the protection attenuates the major part of the impact energy and the cranial vault remains unaltered. Only a significant deformation is manifested in the scalp without leading to its rupture. For this same case, Sarron et al. report skin injuries but no skull fracture. The discrepance is reasonable as the developed head model considers scalp tissue as a linear elastic material for computational savings, and no deep study has been performed in order to establish proper failure thresholds able to account for the smallest injury manifestations (for example, lacerations). Nonetheless, scalp damage by itself, especially when it is minor or moderate, does not imply further lesions to the skull and intracranial contents and for this reason the fact of not accounting for such events appears an assumable limitation.

Test \#68, with an aramid plate in direct contact with the head, leads to the most unfavorable situation. In fact, the simulated results reveal that the bullet almost perforates the plate. Consequently, a depressed circular fracture is obtained in the head model at the impact site accompanied by a relevant scalp laceration, as can be observed in Fig. 5.12 (middle row and right column). This coincides with the injury severity reported in [222], which labels the lessions as severe for both tissues.

Finally, the reconstruction of test \#72 results in an injury pattern somewhere between the previous two experiments: there is a significant scalp breakage but of lower diameter than in case \#68 and a highly-localised cranial vault fracture located just below the laceration. As opposed to \#68, the skull fracture does not extend beyond the damaged scalp area. In [222], these injuries are classified as moderate, a level which we can consider in agreement with the numerical results presented here.

In order to obtain a quantitative approach of the correspondance between experiment and simulation, the numerical cisternal pressures were measured for each case (which are reported in Table 5.6), and found to be consistently close to the experimental values. 

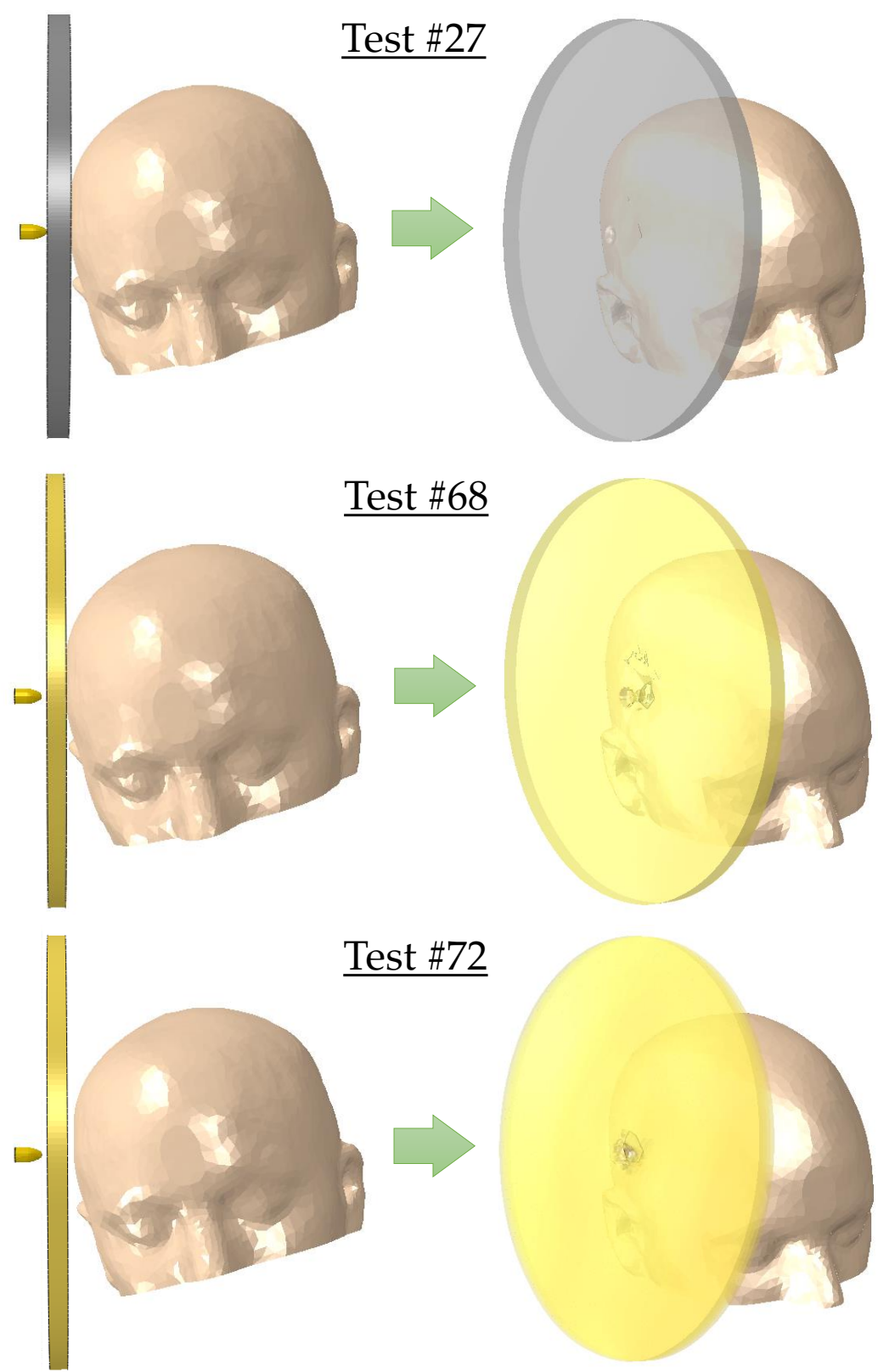

Figure 5.12: Initial and final states in the Abaqus/Explicit simulation of the experimental ballistic tests (number \#27, \#68 and \#72) from Sarron et al. [222] on human cadaveric heads. 


\begin{tabular}{lllllll}
\hline $\begin{array}{l}\text { Test } \\
\text { ID }\end{array}$ & $\begin{array}{l}\text { Plate-head } \\
\text { distance } \\
(\mathrm{mm})\end{array}$ & $\begin{array}{l}\text { Protective } \\
\text { material }\end{array}$ & $\begin{array}{l}\text { Plate } \\
\text { thickness } \\
(\mathrm{mm})\end{array}$ & $\begin{array}{l}\text { Bullet } \\
\text { velocity } \\
\left(\mathrm{ms}^{-1}\right)\end{array}$ & $\begin{array}{l}\text { Experiment } \\
\text { cisternal } \\
\text { pressure } \\
(\mathrm{kPa})\end{array}$ & $\begin{array}{l}\text { Model } \\
\text { cisternal } \\
\text { pressure } \\
(\mathrm{kPa})\end{array}$ \\
\hline 27 & 0 & Aluminum & 12.5 & 383 & 40 & 52 \\
68 & 0 & Aramid & 9.7 & 388 & 193 & 180 \\
72 & 8 & Aramid & 9.7 & 386 & 25 & 26 \\
\hline
\end{tabular}

Table 5.6: Test conditions for the simulated experiments from Sarron et al. [222] and predicted intracranial (cisternal) pressures after impact by the numerical model from this Thesis.

\subsection{Model limitations}

Along this chapter a finite element human head model has been developed and validated against certain experimental tests from the literature. The purpose of this numerical model is, however, not to reconstruct experimental data but to predict the behaviour of the different head tissues under certain impact conditions. Specifically, the trauma associated to ballistic impact on a helmeted head will be studied. Nonetheless, before carrying out further analyses it is necessary to identify the current limitations of the model and therefore be able to be critical about the subsequent results and do not draw uncertain conclusions.

- First, as explained in the introduction of the chapter, the model developed considers a division of the human head into six homogeneous tissues. For the scalp and cranial bone layers, even being all highly nonhomogeneous in their microstructure, at the macroscale it is reasonable to assume they behave as isotropic materials. Nonetheless, initiation and propagation of failure, which are highly localised phenomena at the micro level, cannot be determined with this macro approach. 
- A similar limitation applies for the CSF structure and the brain, which are constituted by different substructures with distinct geometries and material properties. It is true, as discussed in the introduction, that there is a high dispersion in the mechanical properties of gray and white matter reported in the literature and the event of white matter anisotropy due to axonal alignment is not fully demonstrated. Although the model developed here has demonstrated good ICP prediction, it remains unclear that other variables, such as strain, can be accurately evaluated.

- An inherent limitation of the model is that its geometry is specific from one subject. Therefore, parameters such as skull dimensions, thickness of each tissue layer or head mass may lead to different results. For instance, a thicker skull would have shown a stiffer response when simulating the experiment by Yoganandan et al. [21]. 



\section{Chapter 6}

\section{Head and helmet coupling under ballistic testing}

The study of personal protections is increasingly relying on numerical models to assess the safety performances of the different devices. As discussed on previous chapters, finite element models allow one to overcome the limitations of experimental testing, which include:

- Dealing with post mortem subjects, that involves both an ethical conflict and also a loss of accuracy of the actual behaviour of the living tissues as a result of both dehydration and cease in blood flow.

- Employment of human surrogates equiped with a certain number of sensors, normally accelerometers or load cells. Even though these surrogates (dummies) are constantly being improved in order to resemble a real human head, the simulant materials employed are still not able to fully mimic the response of the human living tissues. Additionally, the set of measurable output signals is reduced due to limitations in the acquisition systems, and normally only acceleration data (and contact pressure in the newest devices) can be extracted from the experiments, which makes it difficult to establish adequate biomechanical tolerances for helmet performance.

- A high economical cost is involved with this kind of procedures. An important infrastructure is necessary to conduct ballistic or blast experiments, including devices to generate the threat (shots, pressure waves) 
and the equipment and sensoring to gather the results (accelerometers, load cells, high-speed cameras, etc).

This part of the Thesis is dedicated to the study of the protection provided by combat helmets under ballistic loading conditions. Specifically, the focus will be set on a specific injury type which is the Behind Helmet Blunt Trauma (BHBT), caused by the blow delivered from the inner part of the helmet shell (called backface deformation (BFD)) to the head [223-225]. This nonpenetrating injury is the consequence of the large deformations that composite combat helmets can sustain without allowing bullet perforation.

In this field, there is a reduced amount of published experimental works due to the above mentioned limitations. Nonetheless, FEA studies are increasingly appearing to fill this void. Some of the most relevant works dealing with BHBT have been published along this century. As detailed in the previous chapter, in 2004, Sarron et al. [222], studied the trauma from a lateral ballistic impact on human postmortem skulls and unembalmed heads. Instead of coupling a helmet to the test specimen, plates made up with different materials were placed between the projectile and the subject, leaving a certain offset (stand-off distance). The ammunition employed were 9 mm-caliber FMJ bullets at velocities about $400 \mathrm{~ms}^{-1}$. BHBT on the parietal head side and using the same ammunition was posteriorly studied by Rafaels et al. (2015) [226]. To this purpose, they employed seven PMHS wearing ultra-high molecular weight polyethylene (UHMWPE) helmets. Their ballistic tests at bullet speeds between 404.9 and $459 \mathrm{~ms}^{-1}$, were classified in three velocity ranges, obtaining minor or non injury for the lowest velocities, mild lesions at intermediate velocities, and mild-to-severe injury at the fastest.

Frontal blunt ballistic injury was assessed by Freitas et al. (2014) [227] using human head surrogates which combined PMHS refreshed skulls and synthetic soft tissues. The protection employed consisted on Lightweight Combat Helmets (LWH) with two different distributions of the internal cushioning, either with 5 pads (4 lateral and one superior) or 7 pads (adding one at the frontal and occipital sites). Their experimental tests comprised different projectile types, one of them being the $9 \mathrm{~mm}$ FMJ that will be employed in this study. For the latter, no relevant injury was found at their test conditions (approximately $438 \mathrm{~ms}^{-1}$ ) with the 7-pad configuration, whereas moderate cranial fractures were obtained when the frontal pad was absent. 
Many of the BHBT studies employing FEA are focused more on blast loading rather than ballistics. Despite some injury mechanisms can be similar between both threats, only the works dealing with bullet impact will be taken into account for this chapter. In this category, one of the first numerical-based studies was conducted by Aare and Kleiven (2007) [228] employing the human head model from Kleiven and Von Holst (2002) [229]. The head model was protected with a combat helmet with the geometrical features of the Personnel Armour System Ground Troops (PASGT), even though the stiffness of the composite shell was varied in order to study its influence on head trauma. The FMJ bullets were modeled as a rigid body with an imposed initial velocity of $360 \mathrm{~ms}^{-1}$ and different angles of incidence. One of their relevant findings on BHBT was that impacts with the bullet perpendicular to the helmet surface led to higher skull stresses while at a certain angle of incidence the shear strains at the brain tissue were increased. Also, it was inferred that stiffer helmet shells lowered the stress levels in the skull but increased brain strains.

One issue some studies have focused on is the acceptance protocols for combat helmets, due to its relevance on ensuring a certain certified level of protection. Following this line, Li et al. (2015) [230] simulated the National Institute of Justice (NIJ) testing protocol for combat helmets employing an aramid-based Advanced Combat Helmet $(\mathrm{ACH})$. This same approach was taken by Rodríguez-Millán et al. [231]. On both works the helmet model is mounted on a headform with a clay core, as stated by the standard [232]. Further details about this testing procedure will be given along the following sections of this chapter. In a recent work by Li et al. [233], the dummy headform was replaced by a human head numerical model developed by Kleiven (2007) [234] in order to quantify the response of the head tissues when varying different constructive parameters of the helmet model.

\subsection{Helmet model}

\subsubsection{Helmet composite shell}

Based on the geometry of an Advanced Combat Helmet (ACH), a baseline numerical model consisting of four homogenised composite layers is developed, as depicted in Fig. 6.1. Eight-node hexahedra (C3D8 in Abaqus) are employed for the meshing process, resulting in a 2578-element mesh. 


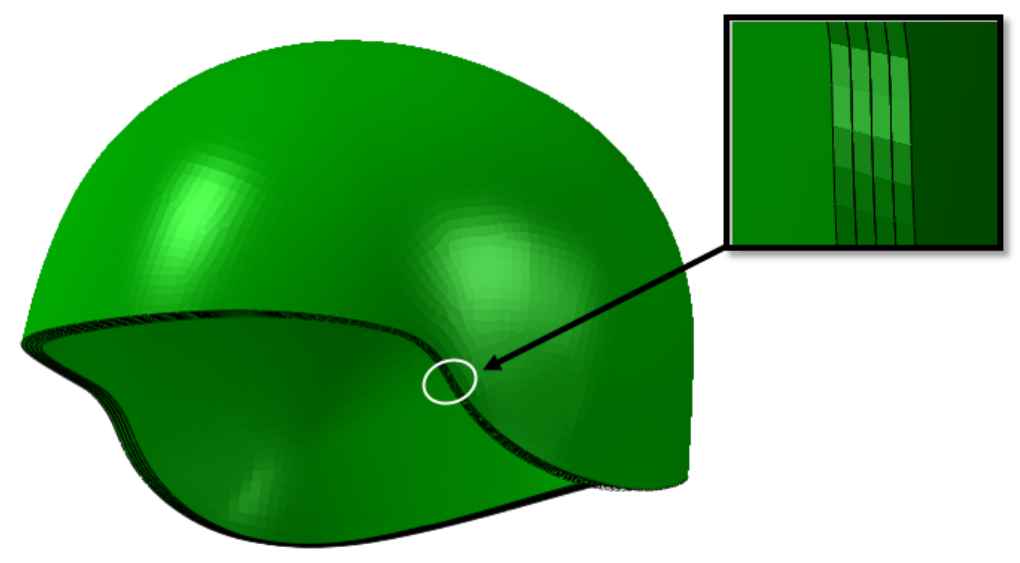

Figure 6.1: Four-layered numerical model of the helmet shell employed in this study.

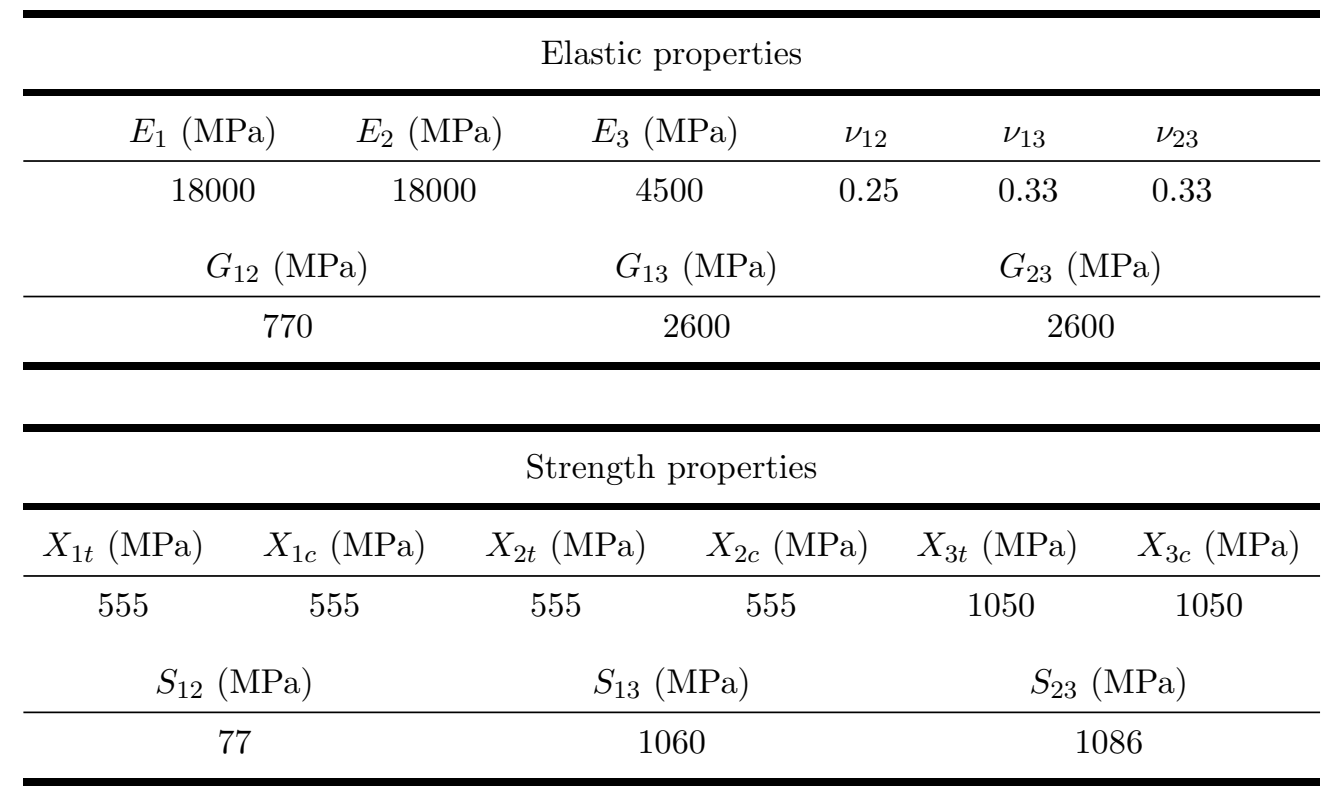

Table 6.1: Summary of the mechanical properties of the aramid fabric layers conforming the helmet shell. 
An aramid woven-fabric material behaviour is applied for each of the shell layers. Its mechanical and resistant properties are summarised in Table 6.1.

The mechanical behaviour of the helmet shell will be governed by a userdefined material through a Fortran subroutine implemented in Abaqus (VUMAT). The algorithm updates the material properties of each element at each iteration according to its stress level. Damage and failure of the composite shell are therefore assessed through the Hashin criterion for fabrics, defining six failure modes, four for the fibers and two for the matrix. The equations governing this failure behaviour are reported in Chapter 4 of this Thesis, as well as the scheme of the VUMAT subroutine into the explicit simulation. Interlaminar damage is assessed by means of a cohesive surface behaviour, whose criterion for initiation is based on the quadratic stress defined in Eq.4.15.

\subsubsection{Interior padding system}

A cushioning system consisting of seven foam pads has been modeled employing the dimensions and geometrical features stated by the Technical Requirements Manual for Combat Helmets. One circular pad is allocated in the bottom of the helmet shell, two lateral rectangular pads are positioned at each side wall and one pad is protecting the frontal region and another the occipital one, as can be appreciated in Fig. 6.2. The meshing is performed by means of the Abaqus software and 6008 (C3D8) type hexahedra are obtain for the full system.

According to the aforementioned Spanish standard for combat helmets, only one shell type is manufactured, with fixed dimensions. To overcome the problem of natural variability in head size between subjects, two different thicknesses of the padding system are stated by the standard, distinguishing between two 'helmet' sizes:

- M size: for head circumference values up to $59 \mathrm{~cm}$, the thickness of the foam pads is set to $1.9 \pm 1 \mathrm{~cm}$.

- L size: for head circumference values larger than $59 \mathrm{~cm}$, the thickness is reduced to $1.3 \pm 1 \mathrm{~cm}$.

The material model applied to this cushioning system corresponds to the Low density foam option in Abaqus/Explicit, as it is suitable for highly- 
deformable soft materials and accounts for strain rate dependencies. Stressstrain curves for uniaxial compression and tension at different strain rates are implemented to the material law, and two viscosity parameters are defined as well as a relaxation coefficient. All these parameters match the experimental ballistic response presented in previous works from the literature ([204]).
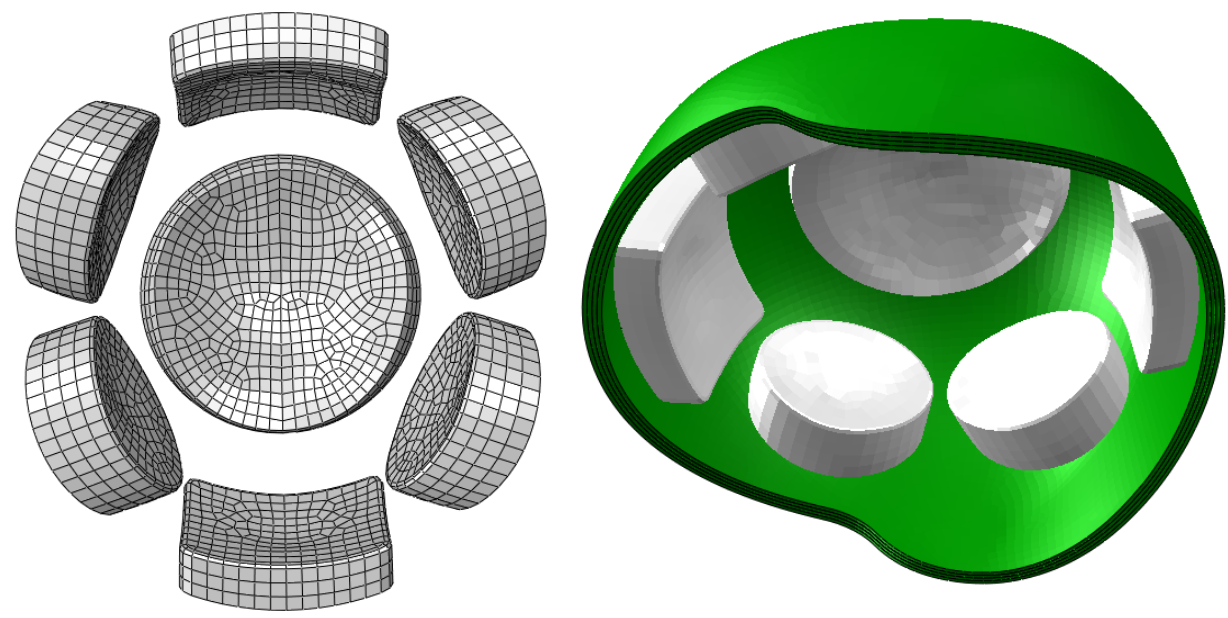

Figure 6.2: Mesh of the modeled foam padding system (left) and its adhesion to the helmet shell (right).

\subsection{Ammunition model}

The ammunition to be employed in the current and the following chapters is a 9 milimeter-caliber bullet of type Full Metal Jacket (FMJ), as illustrated in Fig 6.3 (left). The choice of this kind of ammunition is motivated by the fact that it has been employed in both experimental and numerical works in the literature and therefore it is more suitable to compare results. Furthermore, this bullet is established by many standards as the ammunition to prove ballistic helmet resistance. Specifically, the Spanish standard for the acceptance of combat helmets, which is based on the widely-employed NIJ-STD-0106.01, will be addressed in this study and relies also on the $9 \mathrm{~mm}$ FMJ bullet. 
Based on the geometry of this projectile, a numerical model consisting of a 7777-hexahedra (C3D8) mesh is developed (Fig. 6.3 middle). Two different parts constitute the bullet and are distinguished in the model: a lead core and a brass jacket.
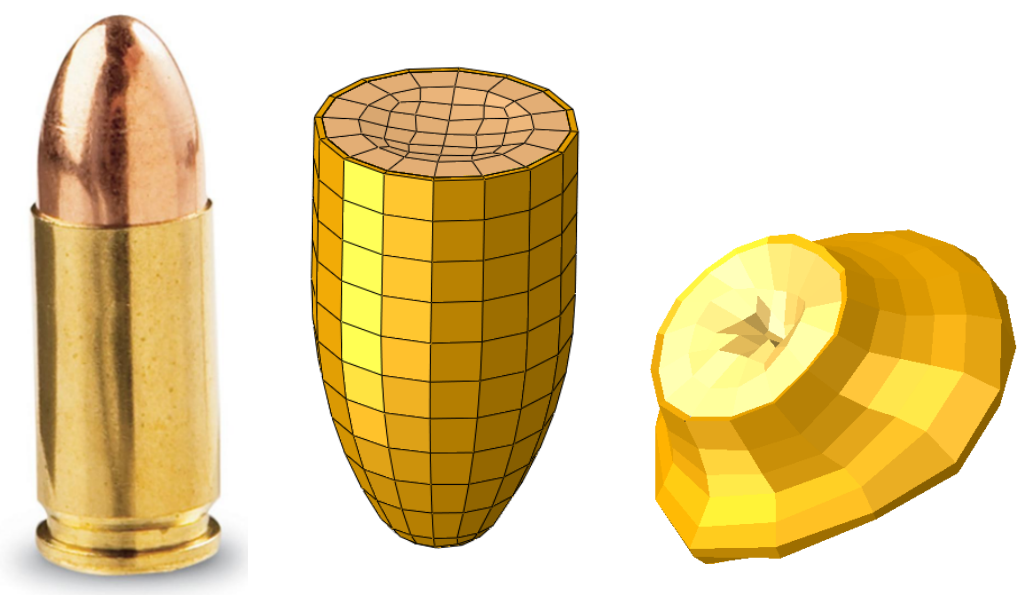

Figure 6.3: Real $9 \mathrm{~mm}$ caliber FMJ bullet (left), mesh for the numerical bullet model including its two parts (middle) and deformed shape after a high-speed impact (right).

The mechanical behaviour of the lead core has been considered to be governed by an equation of state (EOS), whose implementation in Abaqus/Explicit is based on the $U_{s}-U_{p}$ Hugoniot form (Eq.6.2). One assumption of this law is that pressure depends on two parameters: density and the specific internal energy $\left(E_{m}\right)$. This formulation sets a linear relation between the shock wave speed $U_{s}$ and the velocity of the particle $U_{p}$ (Eq.6.1), where $c_{0}$ and $s$ are material-dependent.

$$
\begin{gathered}
U_{s}=c_{0}+s U_{p} \\
p=\frac{\rho_{0} c_{0}^{2} \eta}{(1-s \eta)^{2}}\left(1-\frac{\Gamma_{0} \eta}{2}\right)+\Gamma_{0} \rho_{0} E_{m}
\end{gathered}
$$

All the terms in the right side of Eq. 6.2 are material constants whose 
values for lead are specified in Table 6.2, except for $\eta$, which defines the relation $1-\rho_{0} / \rho$ derived from compressibility effects.

For the brass jacket a viscoplastic material formulation is set. Specifically, a Johnson-Cook law is applied because of its suitability to model the behaviour of metals undergoing high strain rates, and it is complemented with a ductile damage criterion. The formulation is given by Eq. 6.3 and Eq. 6.4 for the Johnson-Cook hardening and damage evolution, respectively. The material constants implemented in Abaqus/Explicit are reported in Table 6.2.

$$
\bar{\sigma}=\underbrace{\left(A+B\left(\bar{\varepsilon}^{p l}\right)^{n}\right)}_{(1)} \underbrace{\left[1+C \ln \left(\frac{\dot{\bar{\varepsilon}}^{p l}}{\dot{\varepsilon}_{0}}\right)\right]}_{(2)} \underbrace{\left[1-\left(\frac{T-T_{a}}{T_{m}-T_{a}}\right)^{m}\right]}_{(3)}
$$

$(2)$

$$
\bar{\varepsilon}_{D}^{p l}=\left(D_{1}+D_{2} \mathrm{e}^{-D_{3} \eta}\right)\left[1+D_{4} \ln \left(\frac{\dot{\bar{\varepsilon}}^{p l}}{\dot{\varepsilon}_{0}}\right)\right]\left(1+D_{5}\left(\frac{T-T_{\text {trans }}}{T_{m}-T_{\text {trans }}}\right)\right)
$$

where

$\begin{array}{ll}\bar{\sigma} & \text { equivalent yield stress (MPa) } \\ \bar{\varepsilon}^{p l} & \text { equivalent plastic strain } \\ \bar{\varepsilon}_{D}^{p l} & \text { equivalent plastic strain after damage initiation } \\ \dot{\bar{\varepsilon}}^{p l} & \text { equivalent plastic strain rate }\left(\mathrm{s}^{-1}\right) \\ \dot{\varepsilon}_{0} & \text { reference plastic strain rate }\left(\mathrm{s}^{-1}\right) \\ \eta & \text { stress triaxiality } \\ T_{m} & \text { melting temperature of the metal }\left({ }^{\circ} \mathrm{C}\right) \\ T_{a} & \text { ambient temperature }\left({ }^{\circ} \mathrm{C}\right) \\ T_{\text {trans }} & \text { transition temperature upon which the material is } \\ & \text { temperature-dependent }\left({ }^{\circ} \mathrm{C}\right) \\ B, C, m, n & \text { model constants experimentally set }\end{array}$

Term (1) of Eq. 6.3 accounts for the effect of strain hardening, while terms (2) and (3) introduce the strain rate dependency and thermal softening, respectively. 
Johnson-Cook model

\begin{tabular}{cccccccc}
\hline $\mathrm{B}(\mathrm{MPa})$ & $\mathrm{C}$ & $\mathrm{m}$ & $\mathrm{n}$ & $\mathrm{A}(\mathrm{MPa})$ & $\dot{\bar{\varepsilon}}_{0}\left(\mathrm{~s}^{-1}\right)$ & $T_{a}\left({ }^{\circ} \mathrm{C}\right)$ & $T_{m}\left({ }^{\circ} \mathrm{C}\right)$ \\
\hline 505 & 0.009 & 1.68 & 0.42 & 112 & 1 & 20 & 1520 \\
\hline \multicolumn{7}{c}{ Johnson-Cook damage evolution } \\
\hline $\mathrm{D}_{1}$ & $\mathrm{D}_{2}$ & $\mathrm{D}_{3}$ & $\mathrm{D}_{4}$ & $\mathrm{D}_{5}$ \\
\hline 0.54 & 4.89 & 0.014 & 3.03 & 1.12 \\
\hline
\end{tabular}

- Lead

\begin{tabular}{cccccc}
\hline$\rho\left(\mathrm{kgm}^{-3}\right)$ & $\mathrm{G}(\mathrm{MPa})$ & $c_{0}\left(\mathrm{~cm}(\mu \mathrm{s})^{-1}\right)$ & $s$ & $\Gamma_{0}$ & $E_{m}$ \\
\hline 11840 & 200 & 0.2006 & 1.429 & 2.6 & 0 \\
\hline
\end{tabular}

Table 6.2: Material constants for the two components of the bullet model.

\subsection{Coupling and simulation conditions}

The baseline model for conducting ballistic simulations consists of the human head model presented in the previous chapter protected with the developed combat helmet. In order to achieve an adequate fit between both parts and ensure full contact between the inner foam pads and the head model, a lowvelocity simulation of the insertion of the helmet into the head is performed in Abaqus/Explicit. This is a realistic approach of a subject putting the helmet on, and it allows to maintain the deformed shape and residual stresses of the foam padding before the ballistic impact. This procedure is schematised in Fig. 6.4.

Contact interactions play a relevant role in this kind of numerical studies and adequate formulations are necessary to avoid undesired effects like interpenetration between elements. Two different contact types from Abaqus are applied in this study:

- The general contact algorithm is chosen to model the interaction between the bullet and the helmet, and between the helmet and the head. 
This choice is justified by the reduction of computational cost with respect to surface-specific interactions, and the fact that self contact in the different parts of the model is taken into account. Furthermore, the general contact algorithm is more robust for the domain discretisation when the calculation is parallelised.

- A surface to surface interaction is set to the interfaces between adjacent helmet layers for which a cohesive behaviour is supposed.

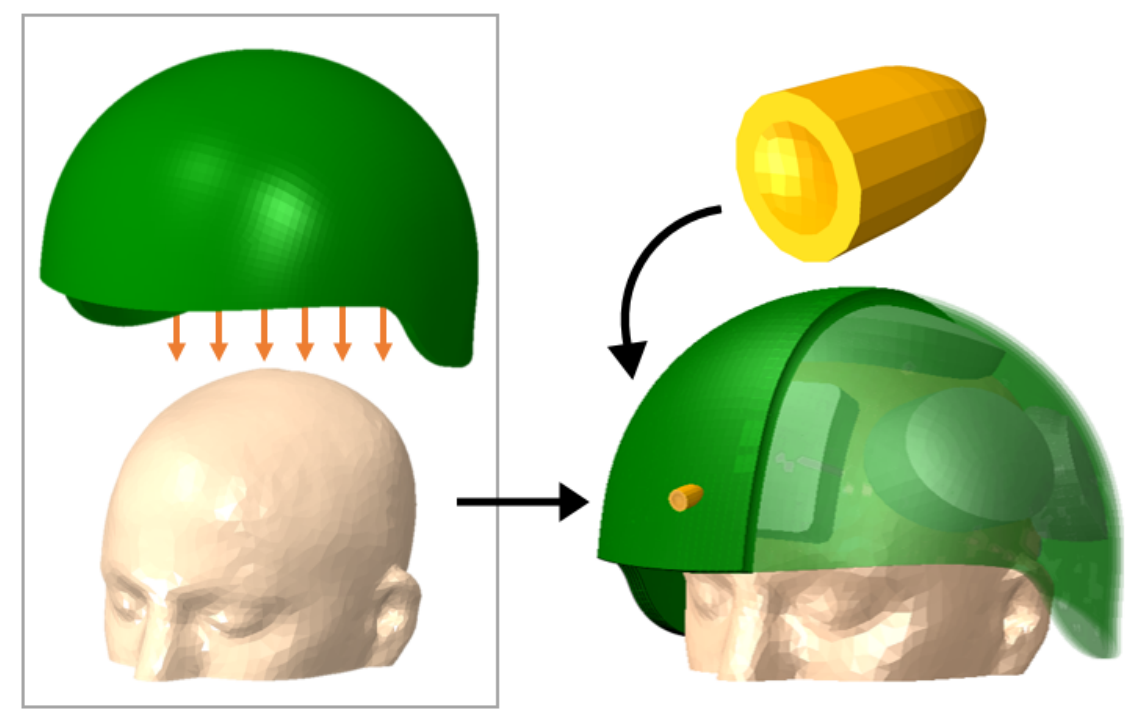

Figure 6.4: Low-velocity insertion of the combat helmet in the head model in Abaqus/Explicit (left), incorporation of the bullet model and final coupled system (right).

Departing from the baseline configuration illustrated in Fig. 6.4 (bottom right), ballistic simulations with different boundary conditions will be performed in order to fulfill three lines of study:

1. The National Institute of Justice (NIJ) standard for the acceptance of combat helmets against conventional ammunition (NIJ-STD-0106.01) will be recreated with the purpose of establishing a relationship between the standard performance requirements and the estimated injury outcome measured from 
the head model. Strengths and deficiencies of this kind of protocols will be put into manifest.

2. A parametric study in terms of the initial velocity of the projectile is carried out in order to assess the evolution of different injury risk indexes when varying the input kinetic energy of the bullet. The protective level of the helmet under these conditions will also be studied.

3. As mentioned in the description of the helmet model, some standards tend to keep a unique helmet shell size and the intersubject differences in head dimensions are contrarrested with the variation of the foam pads thickness. As this fact is likely to affect the performance of the helmet, a study taking two different head model sizes protected with their corresponding helmet is conducted.

In all the simulations performed, the artificial strain energy of the system, which mainly gives a measure of the hourglass energy, is computed and ensured to remain below the $3 \%$ of the total energy.

\subsection{Study of the NIJ Standard (NIJ-STD-0106.01)}

The NIJ standard for combat helmets is one of the most extended regulations for helmet acceptance and many other standards are based on its principles. There are different sets of requirements that a combat helmet has to fulfill regarding various ammunition types and environmental conditions. In this study, only the specifications for helmet resistance against conventional ammunition (9 mm FMJ bullets) will be considered, as this protocol is the one related to head injury prevention. The procedure is the following: the helmet to be tested is mounted on a dummy headform (for which there are a few material possibilities) containing a clay core that has to be in direct contact with the helmet at the striking location. A possible example of this headform is given in Fig. 6.5. The impact response of the headform is restricted in such a way that the only allowable movement is its translation (as a rigid body) in the direction of the shot. Then, ballistic shots at velocities of $358 \pm 15 \mathrm{~ms}^{-1}$ with $9 \mathrm{~mm}$ caliber FMJ bullets are performed in five sites of the head-helmet assembly: one in the frontal and occipital sites, both in the sagittal plane of the head, one at each lateral and one at the vertex position. 


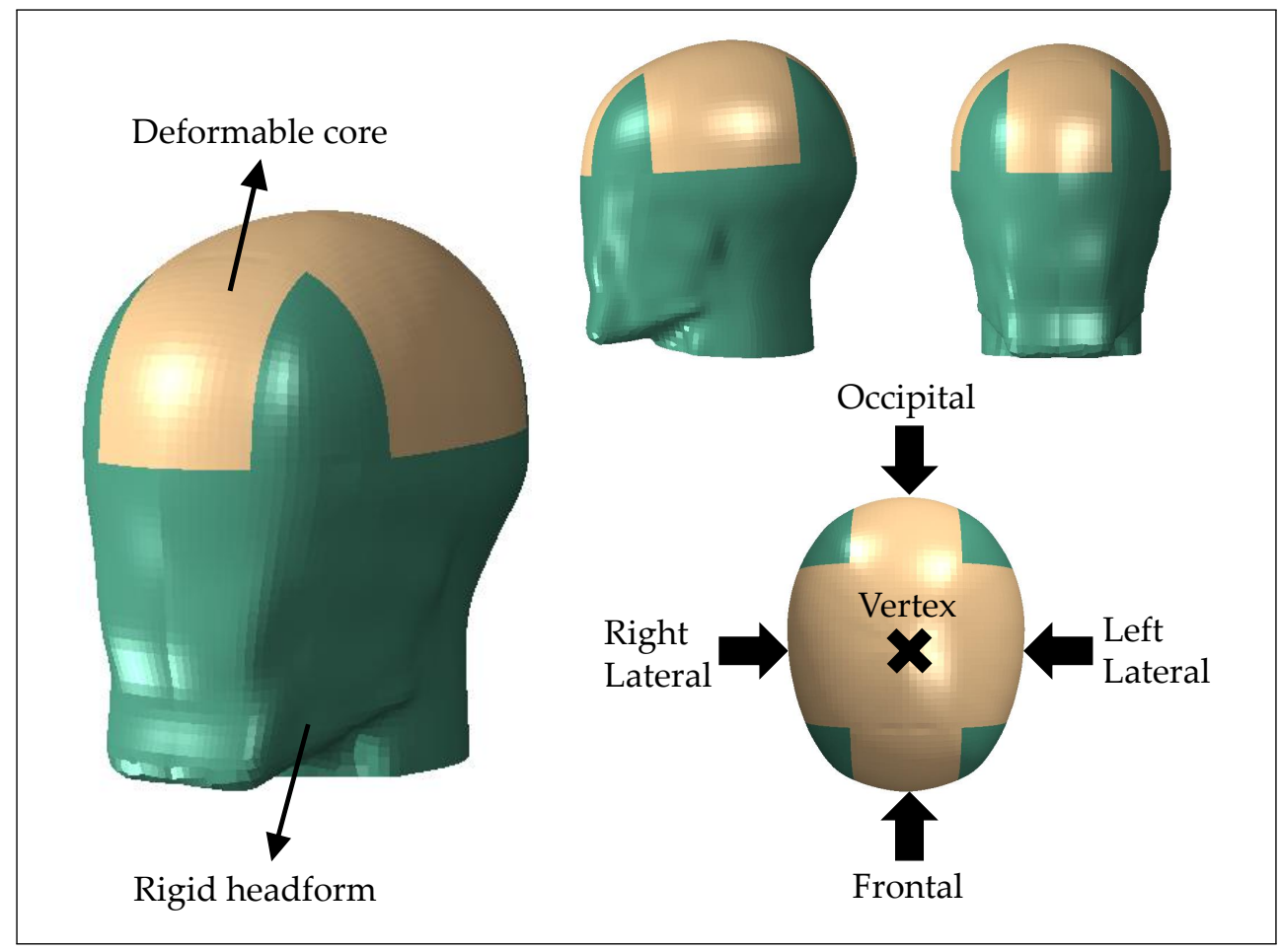

Figure 6.5: Ballistic headform employed for the testing protocol of the NIJ standard. The location of the five compulsory shots with FMJ bullets is schematised in the top view of the headform (bottom right of the figure).

The criterion for acceptance or rejection of the tested helmet is based on the backface deformation (BFD), which is the deformation of the inner layer of the helmet shell at the impact site. It is quantified by the NIJ standard as the permanent deformation left on the clay core of the headform after the shot. Depending on the appliable regulation, the acceptable limit for the BFD varies, although a frequent proposal is about $20 \mathrm{~mm}$. The reason for selecting this parameter as a safety warranty arises from the possible link between the helmet deformation and the magnitude of the blunt blow delivered to the head.

The suitability of this criterion is going to be discussed in this section by means of the simulation of the NIJ protocol conditions on the coupled head/helmet model presented at a previous stage of this chapter. Some head injury metrics will be computed from the developed head model and there- 
fore the correspondence between BFD and head injury risk can be put into manifest.

The baseline assembly for ballistic simulations displayed in Fig. 6.4, which corresponds to a $56 \mathrm{~cm}$-circumference head model protected with a medium size helmet will be employed for the following calculations. A free boundary condition is set to the base of the head model and an initial velocity of 360 $\mathrm{ms}^{-1}$ is applied to the bullet in the instant before the impact.

Fig. 6.6 shows the obtained deformed helmet shell at the moment of maximum BFD value for each of the impact sites stated by the NIJ standard and the computed injury metrics are summarised in Table 6.3. The values highlighted in boldface represent the cases in which the quantity computed from the simulation exceeds the safety threshold proposed in the literature.

\begin{tabular}{lccccc}
\hline & Frontal & Lateral & Occipital & Vertex & Critical values \\
\hline $\mathrm{HIC}_{15}$ & 262.18 & 345 & 285 & 533 & $700[235]$ \\
$\mathrm{SFC}(\mathrm{g})$ & 50.4 & 56.2 & 52.1 & 66.9 & $120[115]$ \\
$\mathrm{p}($ fract $)$ & $5 \%$ & $9.4 \%$ & $6.1 \%$ & $20 \%$ & Measured as \\
& & & & & probability $[112]$ \\
$\mathrm{DDM}$ & $\mathbf{8 9 \%}$ & $\mathbf{9 6 \%}$ & $\mathbf{9 7 \%}$ & $\mathbf{9 9 \%}$ & $7.2 \%[128]$ \\
$\mathrm{CSDM}$ & $0 \%$ & $0 \%$ & $0 \%$ & $0 \%$ & $55 \%[128]$ \\
Peak ICP $(\mathrm{kPa})$ & $\mathbf{2 4 3}$ & $\mathbf{2 5 0}$ & $\mathbf{2 6 0}$ & $\mathbf{4 6 0}$ & $234[141]$ \\
\hline
\end{tabular}

Table 6.3: Obtained values for certain head injury metrics under the simulated ballistic impacts at the locations stated by the NIJ-STD-0106.01. Last column presents critical threshold values taken from the literature.

In view of these results, it is remarkable the difference between the pressurebased indexes and the acceleration-based and strain metrics. The predictor $\mathrm{HIC}_{15}$ has been evaluated along the $15 \mathrm{~ms}$ of the simulation, because it is the lowest time interval for which a certain regulation is established. Nonetheless, the acceleration histories at the center of gravity of the head model only acquire relevant values at about $1 \mathrm{~ms}$ after the beginning of the impact. In fact, after approximately $5 \mathrm{~ms}$ the acceleration values are negligible. This explains the low values of $\mathrm{HIC}_{15}$ and its derivated metrics, as the maximal accelerations 
are averaged over an excessive time lapse. Therefore, this implies that these criteria may not be suitable for the ballistic environment, where the impact effects attenuate more rapidly.

a)

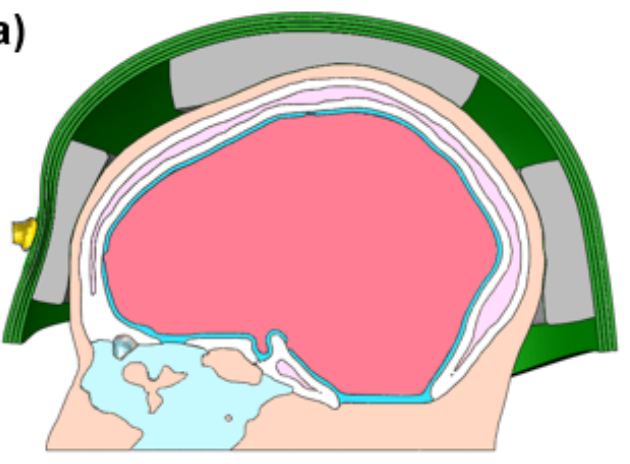

c)

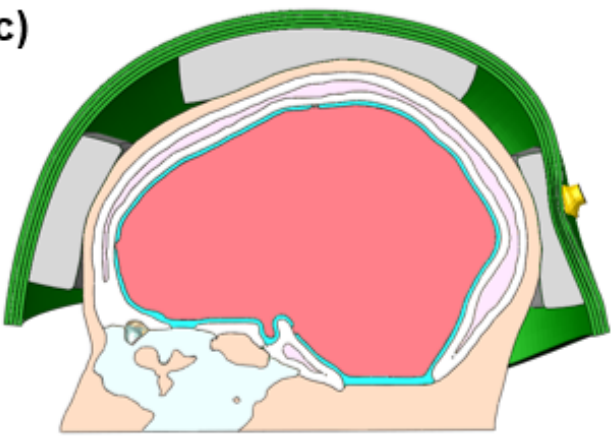

b)

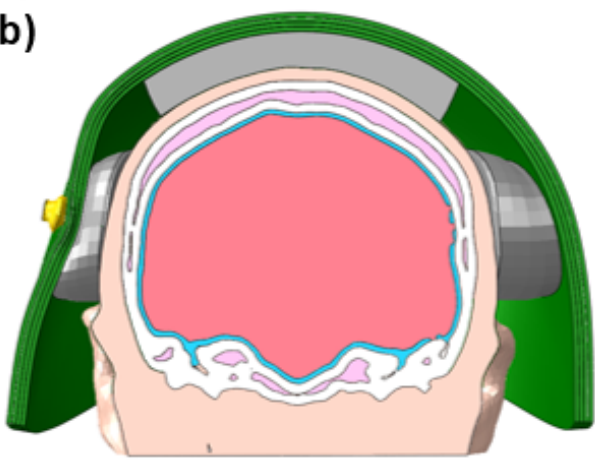

d)

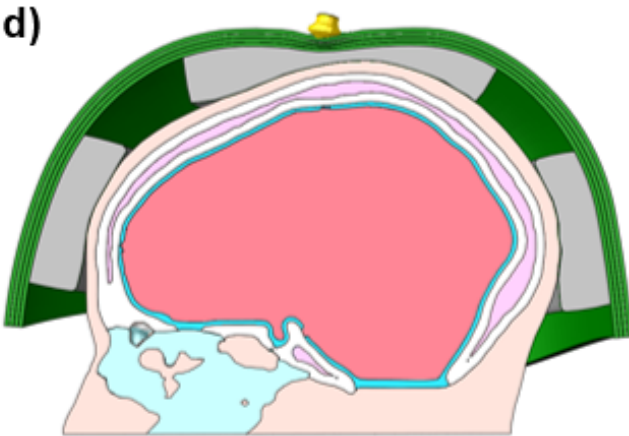

Figure 6.6: Transversal cut in the simulation at the time of maximum BFD for each of the impact sites stated by the standard: a) frontal, b) lateral, c) occipital, d) vertex.

Comparing the acceleration-based metrics for the four impact sites tested, it can be observed that frontal and occipital impacts are less kinematically sensitive than the other two. This assumption applies for the translational kinematics, as the rotational components have not been measured. The vertex appears to be the region subjected to more injury risk, but it is necessary to point out that including a constraint to simulate the effect of the neck may reduce this effect. 
The cumulative strain damage measure (CSDM) is null for the four positions tested. It has been calculated through a Python postprocessing routine, which classifies as injured the elements in the brain tissue exceeding a certain strain threshold value. Once the element has been classified as injured, this condition is irreversible, and in this manner the cumulative volume can be computed. Different strain threshold values have been tested: $20 \%$ (the most commonly stated in the literature for axonal injury), $10 \%$ and $5 \%$. In all cases the resulting CSDM values were 0 , even with the lowest threshold value. This fact suggests that the strain fields in the brain developed from the ballistic impacts stated by the NIJ standard, whose peak values remained in the order of $10^{-3}$ (strain units), are far from reaching injury conditions.

The same methodology employed for computing the CSDM has been followed for the dilatation damage measure (DDM) selecting pressure as the critical parameter. The threshold value has been set in $-100 \mathrm{kPa}$ (approximately the value for cavitation in water). In the four ballistic simulations, the DDM values obtained are one order of magnitude higher than the critical value according to the literature. This implies a risk probability of contusive lessions of almost $100 \%,[128]$. Another issue concerning this results is the possibility of cavitation occuring in the CSF. One limitation of the model employed in this study is that it does not account for such change-of-state mechanisms, and therefore it remains uncertain whether cavitation is occuring as a result of the imparted ballistic impacts. In the case of this phenomenon taking place, it has been suggested in the literature that, when a sufficient volume of liquid has vaporised, cavitation bubbles can form and collapse, affecting the strain levels in the brain tissue. Nonetheless, this 'strain amplification' would not have a determining effect on the brain strain fields obtained here, as an increase of at least one order of magnitude would be necessary for inducing a mild diffuse injury. On the other hand, such high DDM levels are likely to cause damage to the brain vasculature which can lead to intracranial haemorrhages.

Regarding the positive pressures, all computed ICP values are superior than the $234 \mathrm{kPa}$ critical level for severe brain injury determined by Ward et al. [141]. Other authors reported similar peak pressure values in experimental ballistic tests (Sarron et al. [222], Freitas et al. [227]) with the same ammunition but different protections, which supports the results obtained here. Therefore, the model predicts severe contusions under the conditions stated by the standard. 
In conclusion, the head-helmet model developed in this chapter has been submitted to the ballistic impacts stated by the NIJ-STD-0106.01 standard for helmet homologation. According to the specifications of the standard in terms of maximum BFD values, the tested combat helmet accomplishes this requirement in all the impact sites tested. The purpose of this section was to determine the potential injury risk that an accepted combat helmet can impart to the human head. In view of the results presented, the protection against skull fracture and acceleration-derived injuries is adequate, and the risk of sustaining diffuse axonal injuries can be considered as null. The opposite condition is obtained for the ICP-based metrics, fact that reveals a great risk for the occurrence of brain contusions and haemorrhages.

This study aims to give some insight to the combat helmet testing protocols and their strengths and limitations. It has been demonstrated that whereas a helmet can satisfy the BFD requirements, it is not necessarily capable of preventing all possible head injury mechanisms. Specifically, the inclusion of pressure-based criteria to these standards is recommended.

\subsection{Effect of projectile initial velocity}

The previous section studied the response of a human head protected with a combat helmet under ballistic simulations. Nonetheless, the impact scenario was limited to the one stated by the NIJ standard. In this section, the evolution of some of the injury mechanisms in the head as well as the response of the combat helmet will be studied employing different initial velocities for the projectile.

In BHBT, the aftermaths of a ballistic impact are dependent on the magnitude of the blow delivered to the head by the helmet shell when it deforms. Therefore, increasing the velocity of the projectile will increase the kinetic energy reaching the head of the subject, but the relation of muzzle speed-injury risk is not so straightforward. The deformation and fracture pattern of the helmet shell will play a determinant role on BHBT, as the energy distribution and dissipation along its layers will affect the resultant area getting in contact with the head.

Simulations will be performed on the $56 \mathrm{~cm}$-circumference head model covered with the M-size helmet. Ballistic shots of $9 \mathrm{~mm}$ FMJ bullets will be 
delivered to the frontal and lateral sites at velocity values ranging from 425 $\mathrm{ms}^{-1}$ up to the limit velocity that causes the helmet shell perforation.

\subsubsection{Performance of the helmet shell}

For each ballistic impact, the maximum BFD of the helmet shell has been recorded. Figs. 6.7 and 6.8 illustrate these deformed configurations by means of a cut in the sagittal and coronal planes, respectively, in order to show a more detailed view of the damage evolution taking place in the composite shell with the increasing bullet speed.

In the case of frontal shot, the maximum BFD value remains less than the stand-off distance between the helmet and the head up to projectile velocities about $500 \mathrm{~ms}^{-1}$. In this range, damage in the composite shell occurs at the site of impact and a permanent deformation is left at the striking surface, but no macroscopical fiber failure is reached. Beyond this point, the helmet layers begin to break, starting from the inner to the outer surface.

After the failure of the first inner layer, the relation between BFD and impact velocity becomes steeper until the complete perforation of the shell, which is achieved at a bullet velocity of $635 \mathrm{~ms}^{-1}$.

In the lateral case, the stand-off distance is greater, and there is no helmethead contact up to a projectile velocity of about $540 \mathrm{~ms}^{-1}$. 


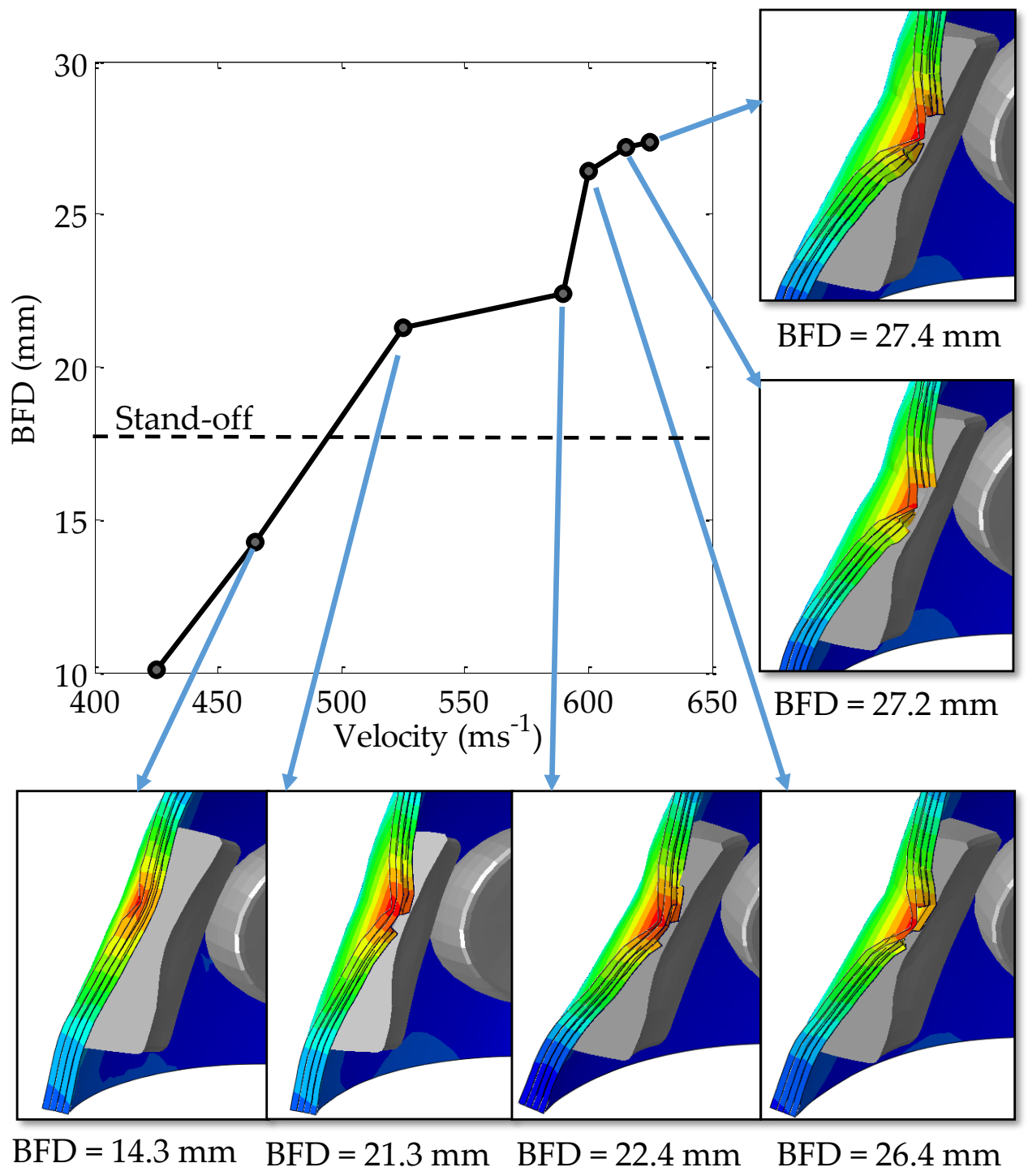

Figure 6.7: Evolution of the maximum BFD registered in the numerical helmet model at different projectile velocities under frontal impact conditions. 


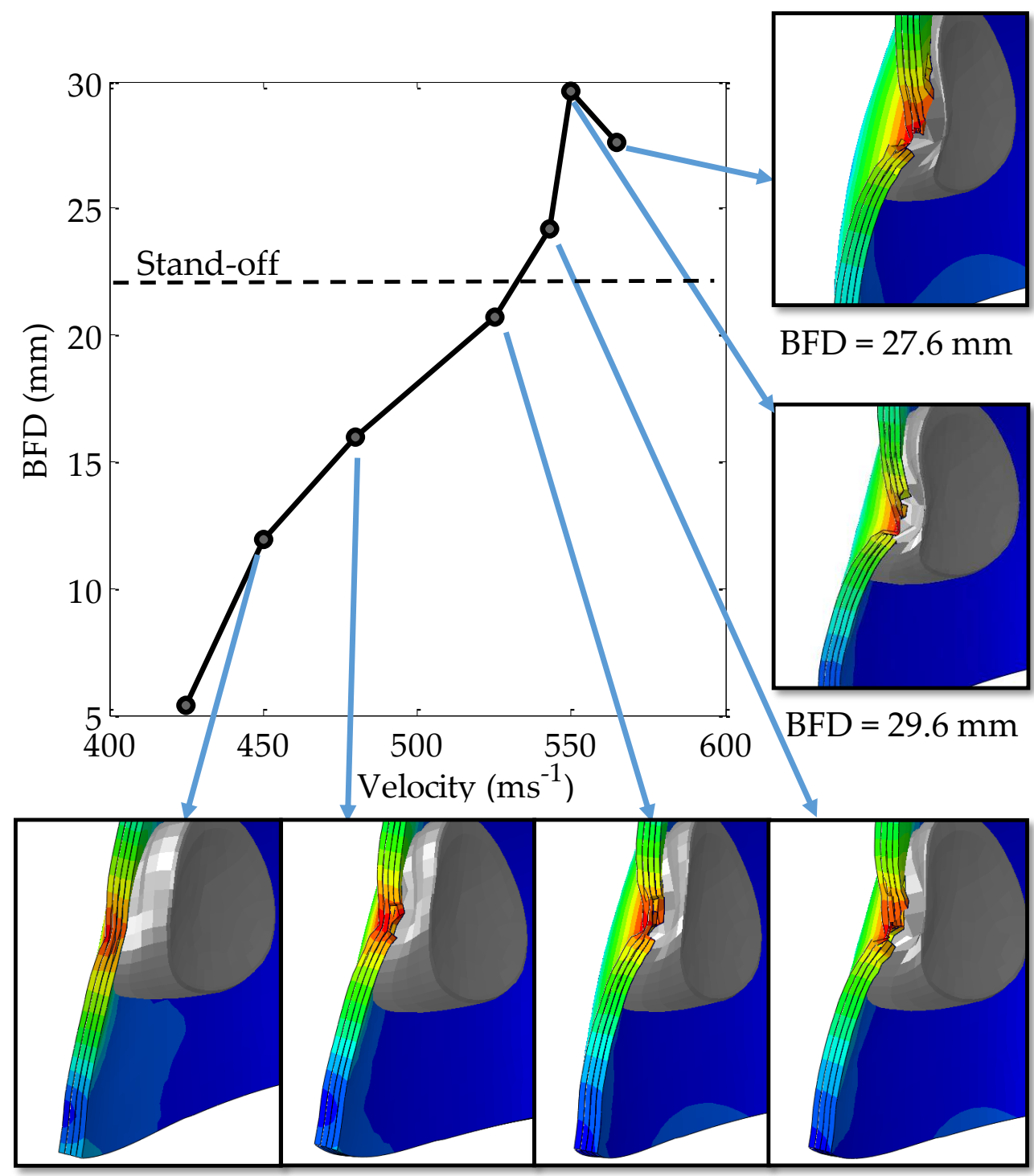

$\mathrm{BFD}=11.9 \mathrm{~mm} \quad \mathrm{BFD}=16.0 \mathrm{~mm} \quad \mathrm{BFD}=20.7 \mathrm{~mm} \quad B F D=24.2 \mathrm{~mm}$

Figure 6.8: Evolution of the maximum BFD registered in the numerical helmet model at different projectile velocities under lateral (temporoparietal) impact conditions. 


\subsubsection{Assessment of head trauma}

After discussing the response of the helmet shell depending on the impact velocity and location, the resulting aftermaths on the head model have been analysed.

The most noticeable trauma type is skull fracture, and in this section it will be taken as the main risk indicator. Several parameters related to this phenomenon (stresses and strains in the cranial layers) have been examined in order to obtain a metric to relate impact velocity with cranial failure. Good correlation was achieved taking as metric the internal energy (IE) of the skull. This measure is obviously related to the amount of kinetic energy absorbed by the helmet and, therefore, its performance. The peak IE for each velocity is represented in Fig. 6.9 for the frontal (left) and lateral (right) shots.

\section{Crack initiation}
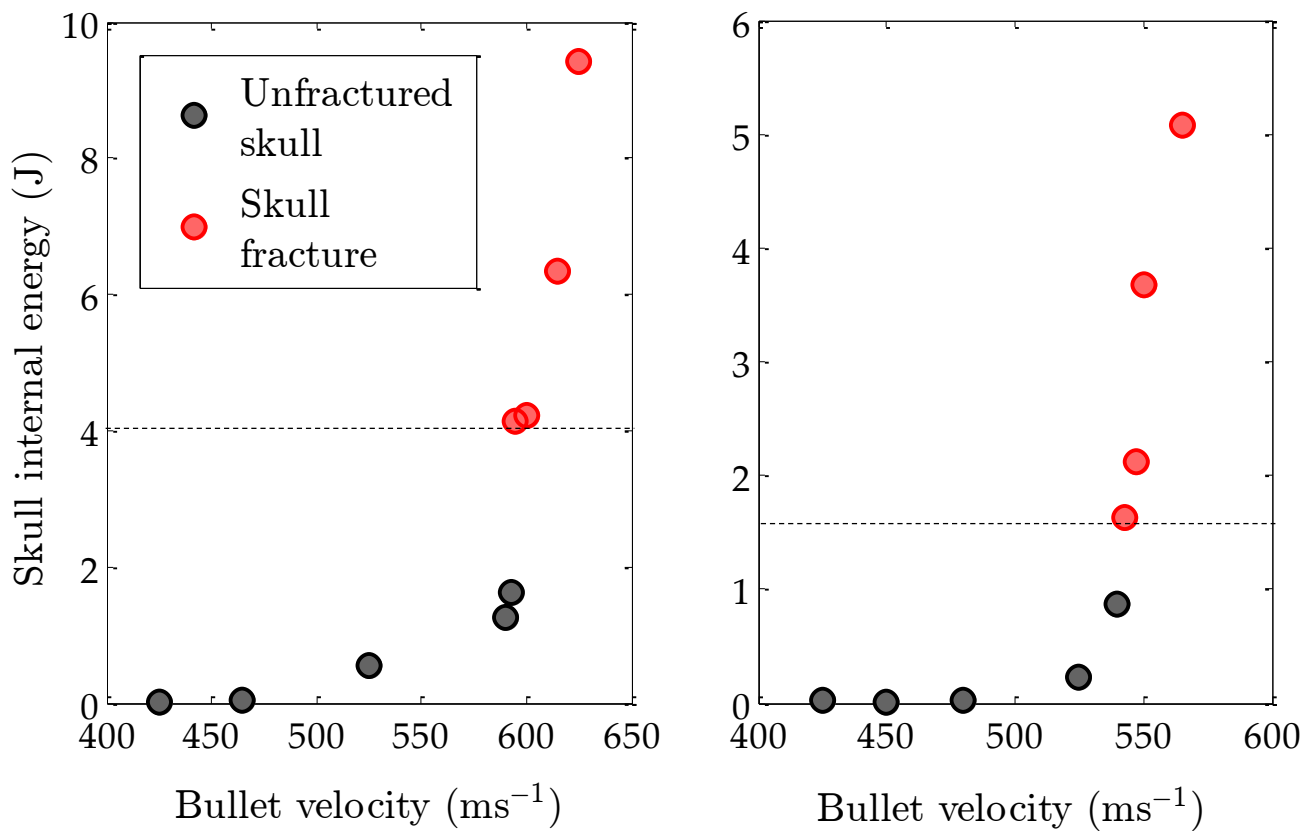

Figure 6.9: Values of the skull internal energy obtained for each simulation at a given initial projectile velocity for frontal (left) and lateral (right) ballistic impacts. 
The internal energy of the skull has previously been considered a suitable parameter to predict fracture, for instance in the work of Sahoo et al. (2016) [148]. As it can be seen in Fig. 6.9, the relation IE-velocity approximates to an exponential law, where the steepest part is initiated in the transition between the cases where the skull was fractured and where it remains unharmed. This finding suggests the existence of a crack initiation energy thershold in this inflection stretch in the curve. For frontal bone, this threshold value lies in the range between 2 and $4 \mathrm{~J}$, whereas for the temporoparietal region it is relatively lower $(1-1.8 \mathrm{~J})$ though in the same order of magnitude.

\section{$V=595 \mathrm{~ms}^{-1}$}

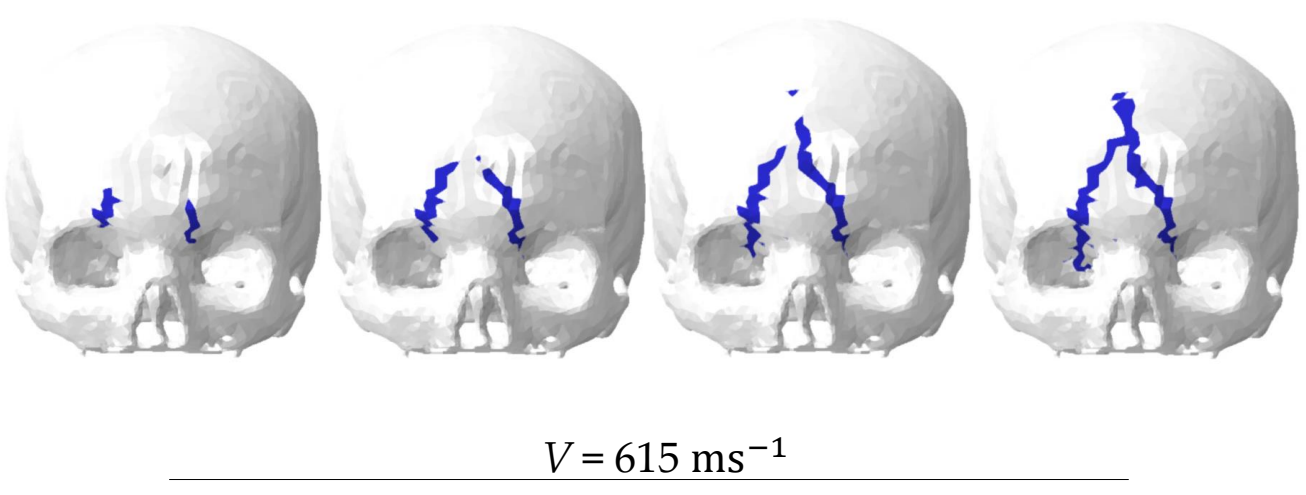

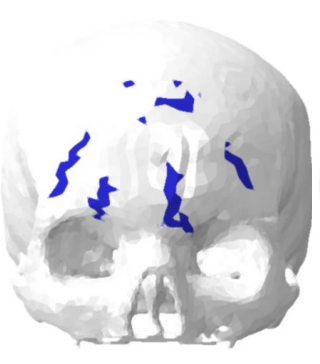

$t=0.21 \mathrm{~ms}$

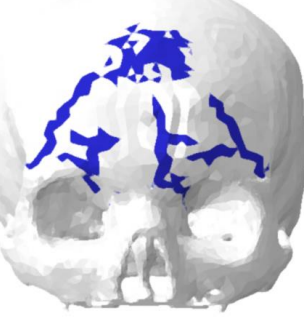

$t=0.24 \mathrm{~ms}$

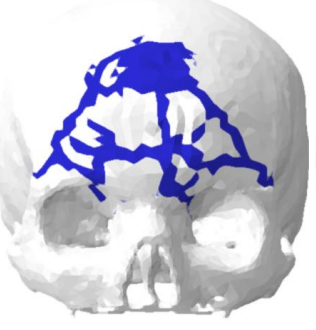

$t=0.28 \mathrm{~ms}$

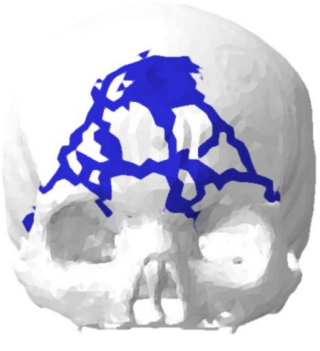

$t=0.42 \mathrm{~ms}$

Figure 6.10: Time evolution of a linear skull fracture at the frontal head site from an impact velocity of $595 \mathrm{~ms}^{-1}$ (top) and stellate fracture caused by an impact at $615 \mathrm{~ms}^{-1}$ (bottom).

These energy values obtained from the ballistic simulation differ by an or- 
der of magnitude from other studies in the literature. For instance, Sahoo et al. [148] suggested a threshold value for cranial fracture of $453 \mathrm{~mJ}$ based on the finite element reconstruction of accidental falls. This discrepancy appears reasonable given the difference in both boundary conditions and loading rate. In fact, no previous study proposes an energy threshold fot skull fracture derived from a behind helmet blunt ballistic impact. Yoganandan et al. [21] and Delye et al. [22] gave mean values for the energy absorbed by the cranial bones under dynamic loads until failure in experimental testing. Their results were, in both cases, in the range between 22 and $24 \mathrm{~J}$, one order of magnitude above the results from the present study. In their works, the impacted cadaveric head was not allowed to move and therefore results are not directly comparable as an unrestrained skull may behave differently, and once again the loading rate is still far from the ballistic range.

In the frontal shot case the transition velocity at which skull fracture is achieved lies about $595 \mathrm{~ms}^{-1}$, and its physical manifestation is the one characteristic for a linear skull fracture, as the cracks begin at regions distinct from the impact site and advance towards the point of maximum loading. This phenomenn can be seen in the temporal evolution of the frontal fracture pattern extracted from the simulation (Fig. 6.10). Because of the steep relation in the asymptotic part of the IE-velocity curve, small increments in the initial velocity of the projectile lead to more severe head trauma. As it can be seen in Fig. 6.10 (bottom row), an increase in speed from 595 to $615 \mathrm{~ms}^{-1}$ triggers a change in the resultant fracture pattern, from a linear to a stellate fracture.

This last finding is relevant when it comes to brain trauma assessment, as a severe breakage of the cranial vault can give rise to other mechanisms of injury affecting the intracranial contents. Even though the occurrence of severe brain injuries does not necessarily imply the presence of skull fractures [94, 236], it is true that the loss of integrity in the cranial vault, especially in depressed skull fractures, can cause additional deformation in the brain tissue. In fact, in the high-velocity fracture cases simulated in this section, CSDM levels (related to strain at the brain tissue) rise with respect to the non-fractured cases from 0 to $8 \%$. Even though not being a critical value, the increase in brain strains may cause the disruption of localised axonal bodies and therefore the risk of minor-to-mild DAI should not be ignored. 


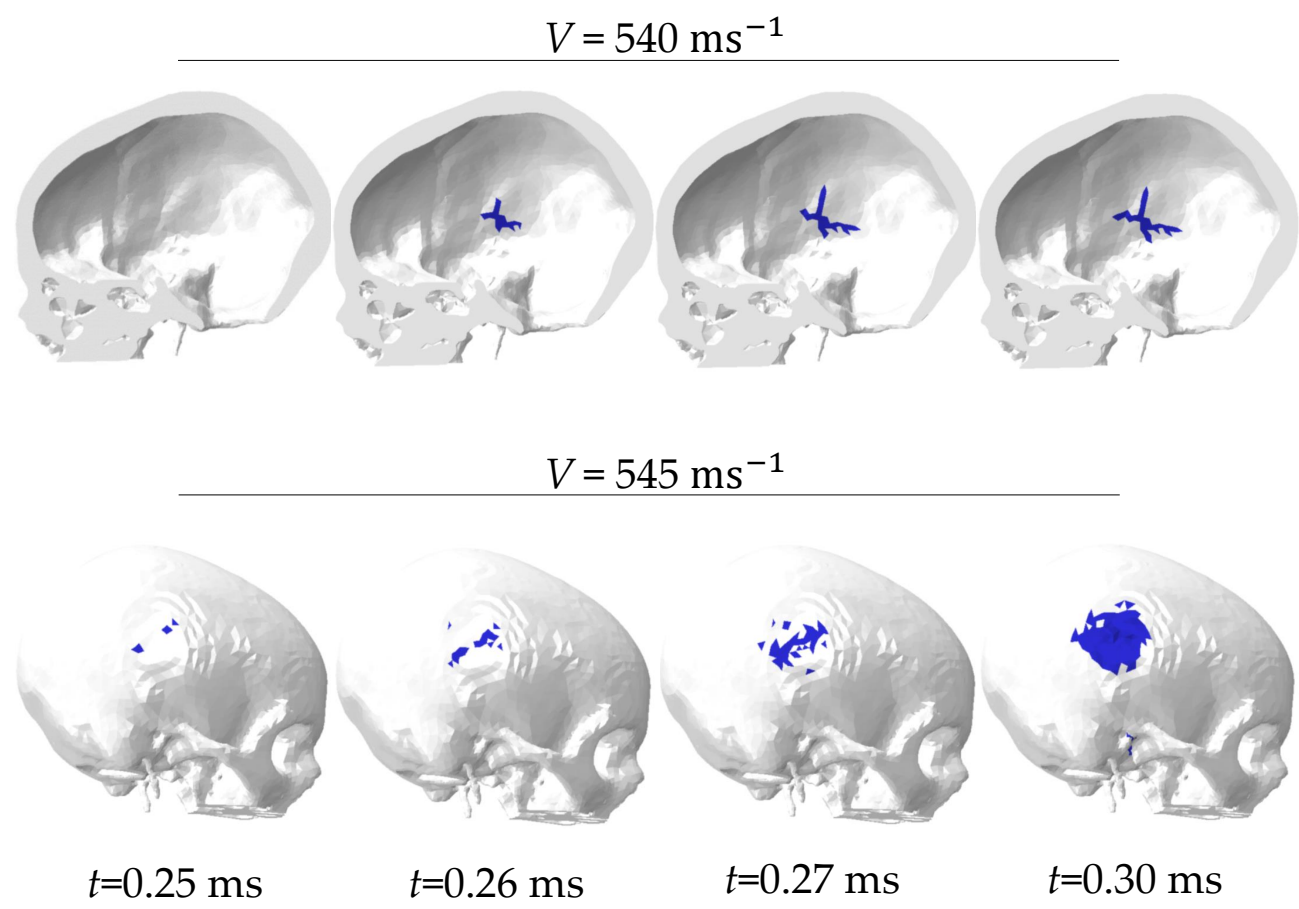

Figure 6.11: Linear cracks at the inner surface of the cranial vault at the temporoparietal region resulting from an impact at $540 \mathrm{~ms}^{-1}$ (top) and depressed skull fracture at the impact site for a velocity of $545 \mathrm{~ms}^{-1}$

As can be appreciated in Fig 6.11, the first internal cracks after the lateral impact start when the skull reaches an energy value of about $1.63 \mathrm{~J}$. This behaviour is more brittle than the observed in the frontal bone, and may be probably explained by the reduction in the thickness of the cancellous bone layer at this lateral region.

The lower resistance of the skull bone in the temporo-parietal region was previously suggested by Zhang et al. [130] in their numerical study on head impact mechanics. They reported greater strain values in these lateral regions with respect to the frontal bone under the same loading conditions. In the ballistic simulations of this study, the critical projectile velocity causing fracture in the skull is about $540 \mathrm{~ms}^{-1}$. At this energy level, the severity of the fracture is minor (Fig. 6.11, top row) and only affects the inner compact bone 
table and the thin diplöe layer, which fail due to bending stesses at the impact site. Analogously to the frontal ballistic impact cases, small projectile velocity increments lead to major trauma consequences. In the lateral case this phenomenon is even more noticeable. As can be observed in Fig 6.11 (bottom), a $5 \mathrm{~ms}^{-1}$ velocity increment causes the occurrence of a depressed fracture that affects the whole cranial thickness whereas minor superficial cracks are found at the middle-posterior part of the skull base. Similar lateral fracture patterns were reported by Sarron et al. [222]. Even though in [222] some of the circular comminuted fractures were accompanied by remote linear fractures, the discrepancy is admissible given the use of different protective materials and stand-off distances.

\subsection{Effect of helmet size}

In the previous sections, the relevance of the stand-off distance has been highlighted. Currently, due to logistic reasons, some helmet manufacturers only produce one type of helmet shell with specified dimensions to cover the full range of head dimensions of the potential customers. In order to gain a better adjustment between head and helmet, the thickness of the padding system is often adapted for different head sizes. As an example, the Spanish standard for helmet manufacturing establishes two sizes ( $M$ and $L$ ) which differ in the thickness of the foam pads, as exposed in the description of the interior padding system model.

Two helmeted-head models will be employed for this study, which are depicted in Fig. 6.12. The top one corresponds to the baseline model employed in the previous two sections, with a head circumference value of $56 \mathrm{~cm}$ and a stand-off distance of $17.8 \mathrm{~mm}$, whereas the model depicted at the bottom corresponds to an L size helmet configuration. The later consists of a head circumference of $61 \mathrm{~cm}$ and a stand-off distance of $12.2 \mathrm{~mm}$. The simulation conditions will be the same from the previous two sections: first, the two helmets will be submitted to the NIJ acceptance protocol and then a parametric study in terms of the initial bullet velocity will be performed. The objective is to compare the different responses of the same helmet shell when the distance to the head is reduced, as it is likely to affect both helmet performance and head injury risk. 


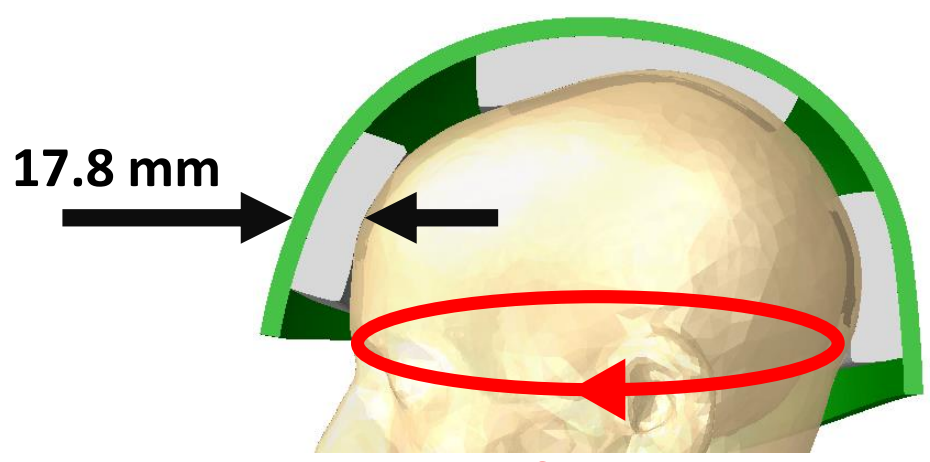

$56 \mathrm{~cm}$

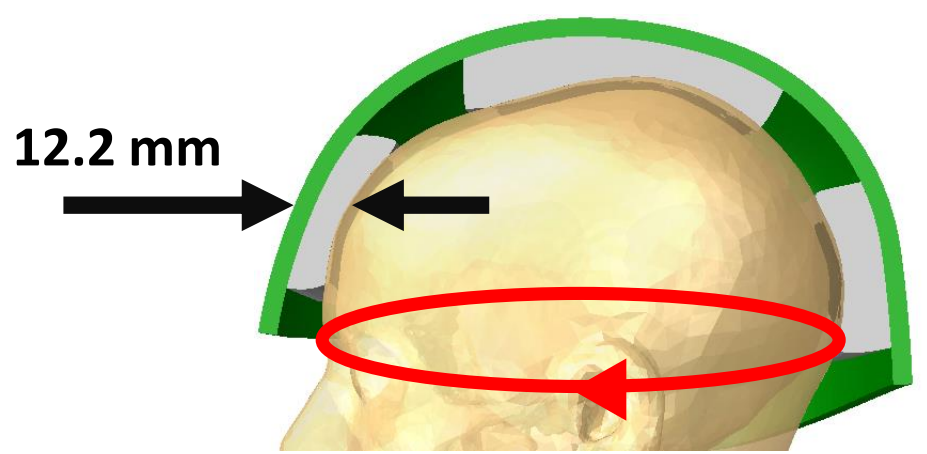

$61 \mathrm{~cm}$

Figure 6.12: M size helmet coupled to a $56 \mathrm{~cm}$ circumference head model (top) and L size helmet protecting a head model whose circumference is $61 \mathrm{~cm}$.

From the simulation of the frontal shot from the NIJ standard, the injury metrics presented in Section 5.4 have been computed and summarised in Table 6.4. As expected, all the risk parameters present greater values when the impact is sustained by the L-size helmet. Regarding CSDM, it remains at a $0 \%$ value as none of the elements in the brain model has exceeded the strain threshold value. However, when measuring the average strains, there is a certain increase in the L-size case with respect to the medium one. 


\begin{tabular}{lccc}
\hline & M-size helmet & L-size helmet & Critical values \\
\hline HIC $_{15}$ & 262.18 & 659.5 & 700 \\
$\mathrm{SFC}(\mathrm{g})$ & 50.4 & 72.8 & 120 \\
$\mathrm{p}($ fract $)$ & $5 \%$ & $28.9 \%$ & $(-)$ \\
$\mathrm{DDM}$ & $89 \%$ & $95 \%$ & $7.2 \%$ \\
$\mathrm{CSDM}$ & $0 \%$ & $0 \%$ & $55 \%$ \\
Peak ICP $(\mathrm{kPa})$ & 243 & 340 & 234 \\
$\mathrm{~V}_{\mathrm{b}}$ for skull fracture $\left(\mathrm{ms}^{-1}\right)$ & 595 & 523 & \\
\hline
\end{tabular}

Table 6.4: Comparison of the injury metrics computed from the FE simulation of the NIJ testing protocol for a medium and a large size configuration.

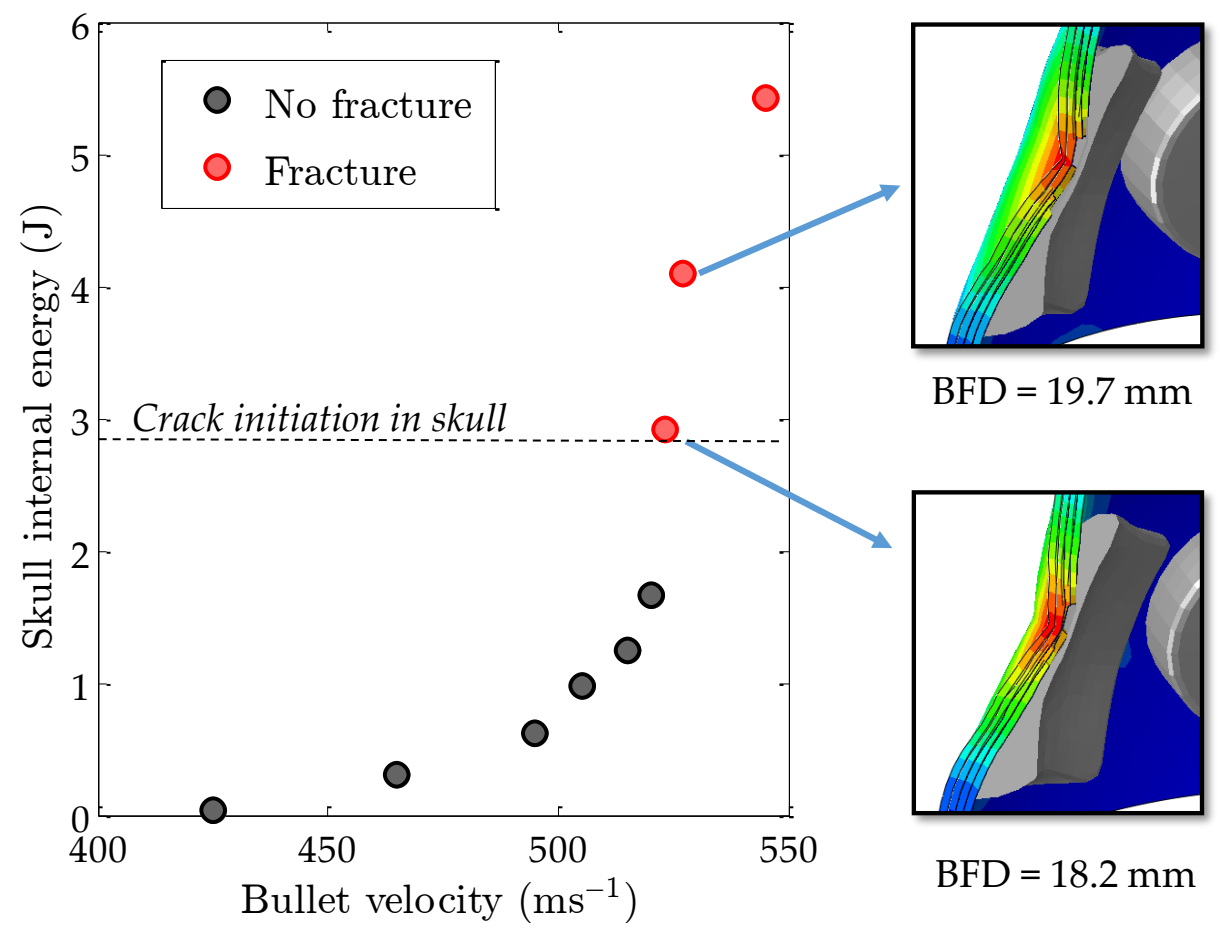

Figure 6.13: Evolution of the internal energy of the skull model for different impact velocities in a frontal shot using an L-size protection. Maximum BFD configurations are represented at the right side. 
Results obtained from the simulations when varying the projectile initial velocity are gathered in Fig. 6.13. The internal energy values to cause skull fracture are consistent with the ones obtained for the frontal shot in the medium size helmet (Fig. 6.7 (left)). Nonetheless, fracture is reached at a lower bullet velocity. Therefore it can be stated that a $30 \%$ padding thickness reduction (corresponding to an $\mathrm{L}$ size with respect to an $\mathrm{M}$ size), with its consequent decrease in the stand-off distance, diminishes by a $13 \%$ the minimal impact velocity to trigger skull fracture. When paying attention to the helmet deformed configurations represented in Fig.6.13, it can be seen that the helmet shell is barely damaged and the BFD values are lower than the obtained for similar velocities with the M-size helmet. This finding suggests the inadequacy of this risk metric as the only criterion for helmet acceptance. As the separation between head and helmet is reduced, the composite shell has less spatial margin to deform without blowing the head and therefore the energy transfer from the helmet to the subject occurs sooner and is greater. This has a double-sided negative effect: it limits the energy absorbing capacity of the helmet and increases the velocity of the BFD region at the time of blunt impact.

The mentioned energy exchange and the fact that at the time of blunt impact the BFD area in contact with the head is greater both have an effect on the resulting fracture characteristics. The temporal evolution of the cranial cracks for bullet velocities of 523 and $530 \mathrm{~ms}^{-1}$ are illustrated in Fig. 6.14. In the first case, the energy level reaching the skull is not sufficient to enhance a severe fracture propagation. Nonetheless, the cracks are located along the base of the skull, causing a basilar skull fracture (BSF), whose criticality is higher than the frontal linear fractures obtained with the $\mathrm{M}$ helmet, as it is related to brainstem injuries and haemorrhages and can cause sudden death [237]. For increased bullet velocities the skull fracture begins at the impact site (frontal bone) and peripheral linear cracks appear at the frontal region and grow towards the adjacent bony areas, some of them propagating from the nasal bone to the base of the skull (Fig. 6.14, bottom). This fracture pattern, clinically named a frontobasal fracture, is specially undesirable due to the possibility of causing, between other aftermaths, coma and sensorial deficiencies [238]. 


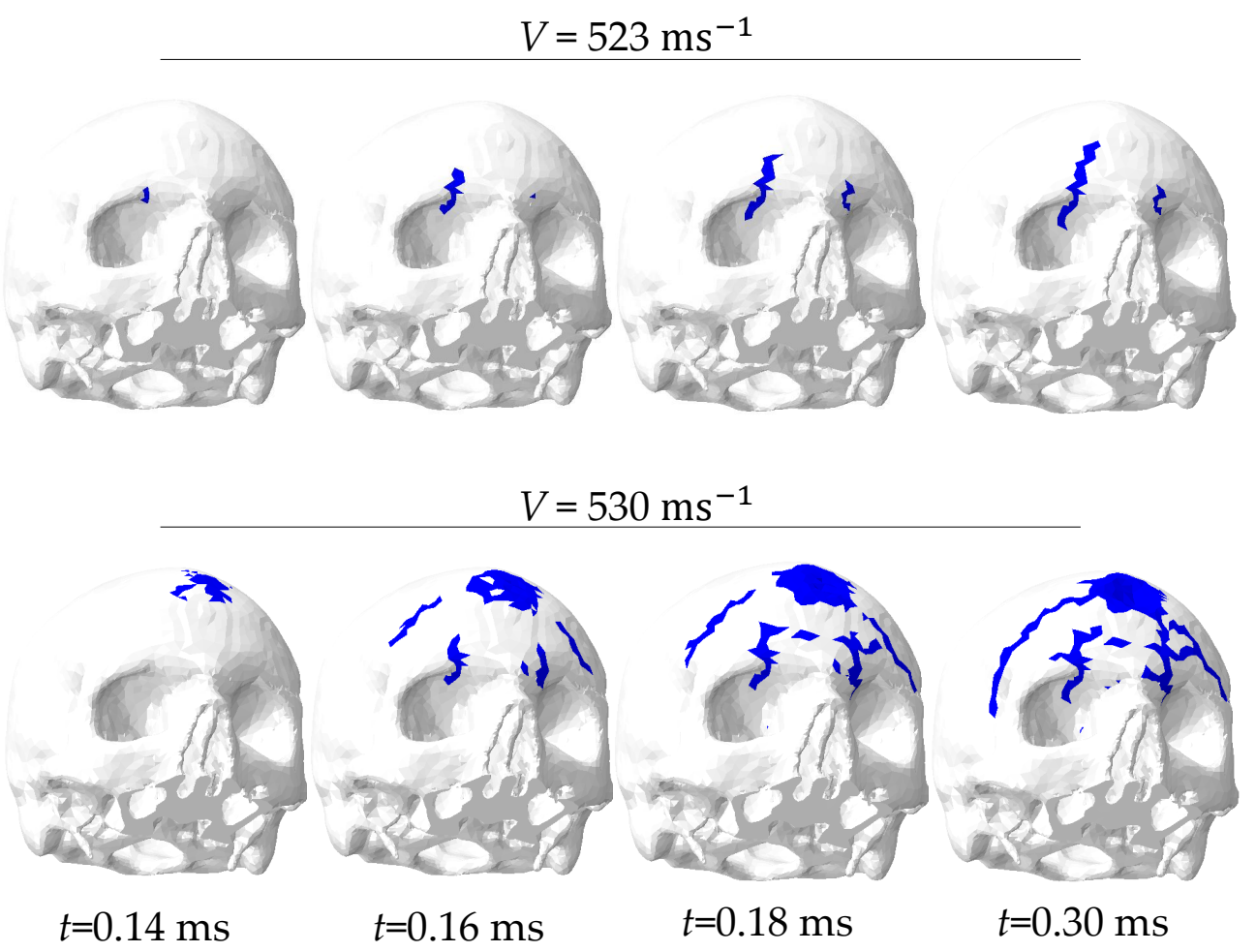

Figure 6.14: Mild (top) and severe (bottom) frontobasal skull fractures caused by frontal ballistic impacts on an L-size helmet configuration at 523 and 530 $\mathrm{ms}^{-1}$, respectively. 


\section{Chapter 7}

\section{Effect of stack configuration of the helmet shell}

Weight reduction is an achievement which has already been desired in composite structures. In fact, this is the focus in many engineering disciplines such as automotion and aeronautics and there is a continuous research and manufacturing effort in order to incorporate lighter materials with good performance.

For instance, this is the case of Ultra High Molecular Weight Polyethylene (UHMWPE), whose strength-to-weight ratio doubles the one of other fibers like carbon. The good energy absorption capability of this material has led to its increasing success in the soft body armour industry, including ballistic helmets $[239,240]$. In fact, it has been claimed in some studies (Karthikeyan et al. (2016) [241]) that UHMWPE panels subjected to ballistic impact can provide a better level of protection in terms of either BFD or $\mathrm{V}_{50}$, depending on their stacking sequence. Thermoplastic polymer-based composite helmet shells, like the one conforming the current Enhanced Combat Helmet (ECH), have proved to have a higher resistance to penetration (normally evaluated through ballistic impacts with low-mass fragments) $[239,241]$ with respect to the aramid-based advanced combat helmet $(\mathrm{ACH})$ helmet. Also, it is said that UHMWPE fibers possess a high wave propagation speed due to their mechanical properties along their longitudinal direction that dissipate the strain waves over wider areas of the material [242]. Nonetheless, as a result of the low shear strength of these laminates, their deformation under the projectile is greater. 
Therefore, the opposite trend to the resistance to penetration is found when evaluating the resulting backface deformation, whose minimisation is a key factor for BHBT prevention.

Achieving a compromise between both ballistic performance parameters has been intended in previous works by combining different ply orientations in the UHMWPE layup. The most commonly commercialised polyethylenereinforced composite panels (such as Dyneema ${ }^{\circledR}$ HB25, HB26) consist of hotpressed layers of unidirectionally aligned fibers at $0^{\circ} / 90^{\circ}$. Even though this configuration enhances a good level of resistance to penetration, alternative orientations of the helmet layers leading to a quasi-isotropic behaviour provide a considerable decrease of BFD [239, 241, 243].

The most recent trends point to the future incorporation of hybrid intralaminar composites, in which bundles of different fibers are woven together in a single ply. For example, UHMWPE/carbon fiber fabrics are regarded to be promising as they enhance a better impact resistance that is not achievable by carbon fiber reinforced materials by themselves [244].

Since the 1980s, several works have studied the impact behaviour of hybrid composites, both experimentally and numerically [245-249]. These works comprise the most widely employed ballistic materials: carbon, glass, aramid and UHMWPE fiber composites. Nonetheless, in most of these studies plate geometries are employed as they are less complex to test experimentally and to validate numerical models. The curved geometry of combat helmets might, however, affect the overall performance of the protective laminate under highspeed impact conditions. Additionally, even though ballistic plates are an adequate approach to estimate the backface deformation of thorax protective panels (on which the curvature is less pronounced), there is a lack of information regarding the protective capacity of hybrid ballistic helmets.

This feature has been taken into account in the study developed in this section. Specifically, the purpose is to evaluate the protective performance of different combinations of the composite materials conforming a combat helmet shell. Both the effect of the stacking sequence of the plies and the materials involved will be assessed by means of frontal shot ballistic simulations. Three different materials, commonly present in current combat helmets, have been taken to conform the shells: aramid fabric, woven carbon/epoxy plies and unidirectional UHMWPE fibers on a polyethylene matrix. Also, the orientations of the fabrics/fibers might be varied, as it will be discussed next. 


\subsection{Shell configurations}

Nine shell configurations have been constructed and simulated under ballistic impact in Abaqus/Explicit. They are summarised in Table 7.1, focusing first on the cases of a single material and then on hybrid lay-ups.

The first two shell configurations consist both of 16 plies of aramid fabric and differ in the orientation sequence. In \#A1, all the fabric layers are oriented at $0^{\circ} / 90^{\circ}$, being $0^{\circ}$ parallel to the sagittal plane of the head at the frontal region. On the contrary, in \#A2 each layer oriented at $0^{\circ} / 90^{\circ}$ is preceeded and followed by another at $45^{\circ} /-45^{\circ}$.

Four UHMWPE-based configurations are also tested in order to address the possibility of certain weight reduction of the helmet shell. Unidirectional UHMWPE plies are employed in these four cases, varying the number of plies and their orientations. The first configuration, denoted as \#P1, is constructed by means of the superposition of unidirectional UHMWPE layers with the reinforcing fibers oriented at $\alpha_{i}+15^{\circ}$, where $\alpha_{i}$ is the angle of the fibers in the preceeding layer. The result is a fiber distribution which resembles an helicoidal pattern, illustrated in Fig. 7.1 (left). A possible reinforcement of this configuration (\#P2) would be to consider each composite layer as a Dyneema HB26 stack. The latter, schematically represented in Fig.7.1 (right), is conformed by alternating plies of UHMWPE fibres oriented at $0^{\circ}$ and $90^{\circ}$ which are stacked by means of hot-pressing [243, 250]. This cross-ply structure enables the assumption of a layer-level orthotropic behaviour [250]. The two remaining polyethylene-based shells consist of a uniform lay-up of HB26 panels with the same orientations with respect to each other. The difference between them is the number of layers, being 36 in case \#P3 and 48 for \#P4.

Finally, four hybrid stack sequences have been studied by placing a few reinforcing plies of a different material on configurations \#A2 and \#P3. In the case of the aramid shell, the reinforcing layers are constituted by woven carbon fiber/epoxy and in the case of UHMWPE, aramid fabric is the complementary material selected. In both cases the reinforcing plates will be placed either at the striking surface (the first in contact with the projectile) or the inner surface of the shell. A schematic view of these hybrid shells is shown in Fig. 7.2. 


\begin{tabular}{|c|c|c|c|}
\hline Config. & Description & $\begin{array}{l}\text { Shell } \\
\text { thickness } \\
{[\mathrm{mm}]}\end{array}$ & $\begin{array}{l}\text { Shell } \\
\text { mass } \\
{[\mathrm{kg}]}\end{array}$ \\
\hline \#A1 & $\begin{array}{l}16 \text { aramid-fabric layers, all with the same } \\
\text { orientation }\end{array}$ & 8 & 1.03 \\
\hline \#A2 & $\begin{array}{l}16 \text { aramid-fabric layers, with alternating ori- } \\
\text { entations of } 0^{\circ} / 90^{\circ} \text { and } 45^{\circ} /-45^{\circ} \text { between adja- } \\
\text { cent plies }\end{array}$ & 8 & 1.03 \\
\hline$\# \mathrm{P} 1$ & $\begin{array}{l}36 \text { layers of unidirectional UHMWPE ori- } \\
\text { ented at } \alpha+15^{\circ} \text { forming an helicoidal pattern }\end{array}$ & 8.64 & 0.888 \\
\hline$\# \mathrm{P} 2$ & $\begin{array}{l}36 \text { layers of Dyneema HB26 oriented at } \\
\alpha+15^{\circ} \text { forming an helicoidal pattern }\end{array}$ & 8.64 & 0.888 \\
\hline \#P3 & $\begin{array}{l}36 \text { layers of Dyneema HB26 with the same } \\
\text { orientations }\end{array}$ & 8.64 & 0.888 \\
\hline$\# \mathrm{P} 4$ & $\begin{array}{l}48 \text { layers of Dyneema HB26 with the same } \\
\text { orientations }\end{array}$ & 11.52 & 1.18 \\
\hline$\# \mathrm{HCA}$ & $\begin{array}{l}16 \text { aramid-fabric layers, with alternating ori- } \\
\text { entations of } 0^{\circ} / 90^{\circ} \text { and } 45^{\circ} /-45^{\circ} \text { between adja- } \\
\text { cent plies and } 2 \text { carbon/epoxy layers at the } \\
\text { striking surface }\end{array}$ & 8.8 & 1.156 \\
\hline$\# \mathrm{HAC}$ & $\begin{array}{l}16 \text { aramid-fabric layers, with alternating ori- } \\
\text { entations of } 0^{\circ} / 90^{\circ} \text { and } 45^{\circ} /-45^{\circ} \text { between adja- } \\
\text { cent plies and } 2 \text { carbon/epoxy layers at the } \\
\text { inner shell surface }\end{array}$ & 8.8 & 1.206 \\
\hline \#HAP & $\begin{array}{l}27 \text { layers of Dyneema HB26 with the same } \\
\text { orientations with } 9 \text { aramid-fabric plies in the } \\
\text { striking surface }\end{array}$ & 8.64 & 1.019 \\
\hline$\# \mathrm{HPA}$ & $\begin{array}{l}27 \text { layers of Dyneema HB26 with the same } \\
\text { orientations with } 9 \text { aramid-fabric plies in the } \\
\text { inner shell surface }\end{array}$ & 8.64 & 1.01 \\
\hline
\end{tabular}

Table 7.1: Different helmet shell configurations tested in this work. 


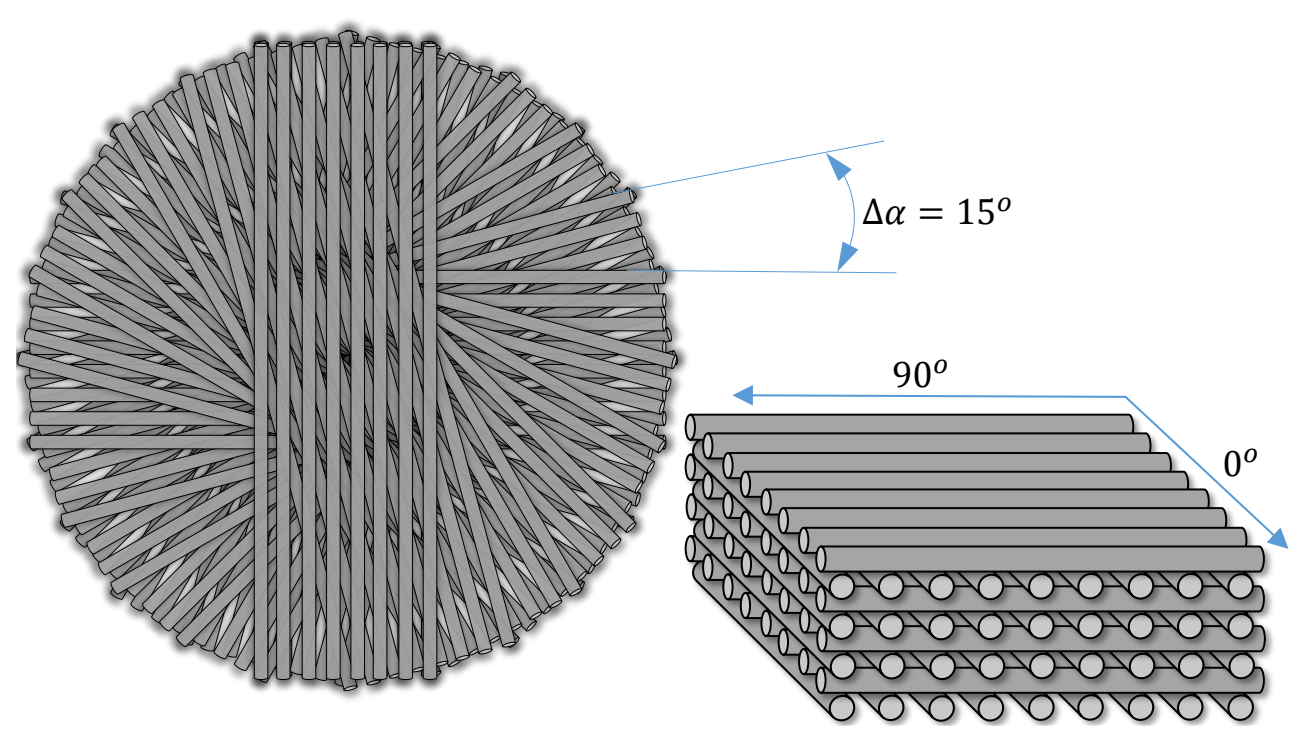

Figure 7.1: Left: helicoidal-pattern lay-up of unidirectional plies for configuration \#P1. Right: scheme of the cross-ply structure of the HB26 stack employed in the modeling of \#P2, \#P3 and \#P4.

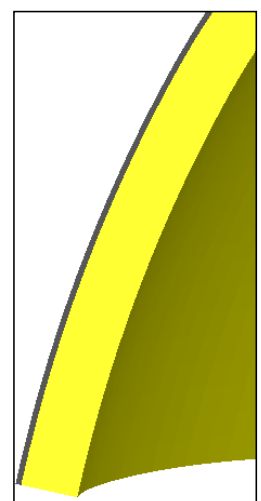

Aramid with carbon fiber
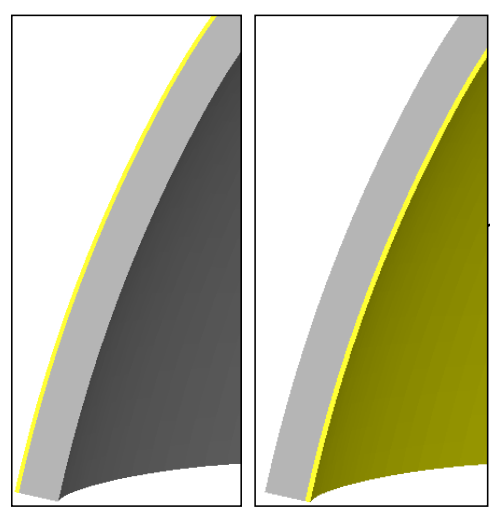

UHMWPE with aramid

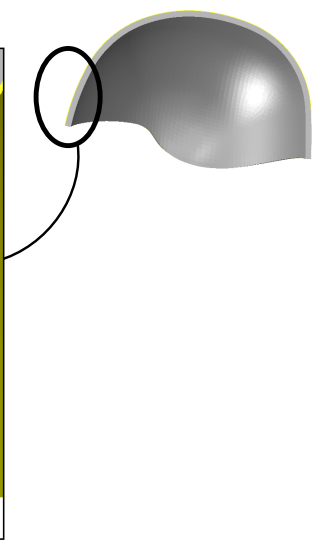

Figure 7.2: Detailed view of the hybrid configurations tested in this study (\#HCA, \#HAC, \#HAP, \#HPA). The reinforcing material is localised whether in the striking or the inner surface. 


\subsection{Material modeling}

The mechanical response of the woven aramid layers is the one explained in the previous chapter, in the description of the ballistic helmet model. It mainly consists of the assumption of an orthotropic material whose elastic and failure properties are listed in Table 6.1. Besides, its failure response is implemented in Abaqus/Explicit by means of a user subroutine based on Hashin's criterion for fabrics, as described in Section 5.1.1.

The CFRP modelled in this section corresponds to a satin fabric carbon fiber/epoxy composite. Its mechanical properties have been obtained from the work by Abot et al. (2004) [251] who reported the mechanical properties summarised in Table 7.2 from impact experimental tests.

Elastic properties

\begin{tabular}{cccccc}
\hline$E_{11}(\mathrm{MPa})$ & $E_{22}(\mathrm{MPa})$ & $E_{33}(\mathrm{MPa})$ & $\nu_{12}$ & $\nu_{13}$ & $\nu_{23}$ \\
\hline 53100 & 53100 & 8000 & 0.055 & 0.074 & 0.074 \\
$G_{12}(\mathrm{MPa})$ & $G_{13}(\mathrm{MPa})$ & & $G_{23}(\mathrm{MPa})$ \\
\hline 3790 & 3700 & \multicolumn{2}{c}{3700} \\
\hline
\end{tabular}

Resistant properties

\begin{tabular}{cccccc}
\hline$X_{11}(\mathrm{MPa})$ & $X_{22}(\mathrm{MPa})$ & $X_{33}(\mathrm{MPa})$ & $S_{12}(\mathrm{MPa})$ & $S_{13}(\mathrm{MPa})$ & $S_{23}(\mathrm{MPa})$ \\
\hline 565 & 565 & 420 & 66 & 66 & 66 \\
\hline
\end{tabular}

Table 7.2: Material properties for the woven CFRP plies of the plate.

The unidirectional UHMWPE layers employed for configuration \#P1 have been modeled based on the work by Yang and Chen (2017) [252]. Their numerical model of the UD laminate is based on the mechanical behaviour of a single ply of the commercial material Dyneema ${ }^{\circledR}$ SB71 which is assumed to be orthotropic and determined by the engineering constants summarised in Table 7.3. Damage and failure are assessed by means of a ductile damage law with an exponential evolution based on energy until complete failure. 
Elastic properties

\begin{tabular}{cccccc}
\hline$E_{11}(\mathrm{MPa})$ & $E_{22}(\mathrm{MPa})$ & $E_{33}(\mathrm{MPa})$ & $\nu_{12}$ & $\nu_{13}$ & $\nu_{23}$ \\
\hline 100000 & 1000 & 1000 & 0.3 & 0.3 & 0.3 \\
$G_{12}(\mathrm{MPa})$ & $G_{13}(\mathrm{MPa})$ & & \multicolumn{2}{c}{$G_{23}(\mathrm{MPa})$} \\
\hline \multicolumn{2}{c}{5000} & 5000 & \multicolumn{3}{c}{500} \\
\hline
\end{tabular}

Damage initiation and evolution

\begin{tabular}{cccc}
\hline Type & Softening & Yield Stress $(\mathrm{MPa})$ & Fracture strain \\
\hline Ductile & Exponential & 1800 & 0.035 \\
\hline
\end{tabular}

Table 7.3: Summary of the mechanical properties of the unidirectional UHMWPE plies from Yang and Chen [252].

The modelling strategy for the UHWMPE panels for \#P2 to \#P4 has been taken from Nguyen et al. [253] as it provides a good agreement between their ballistic tests and their numerical results at a velocity range which includes the impacts studied in this work. As suggested in their work, the material has been discretised in several layers (36) in order to achieve more accurate results both in terms of BFD and energy absorption. The material behaviour of the polyethylene shell is implemented in Abaqus/Explicit using an orthotropic elasticity law in conjunction with an orthotropic plasticity model. Failure is assessed by means of a damage law. In Lässig et al.[250] this same material accounts additionally for an $U_{s}-U_{p}$ equation of state (EOS), but it has not been implemented in the model of this study as the impact velocities remain in the ballistic regime and do not achieve hypervelocity conditions. This assumption was validated in the numerical study by Hazzard et al. (2018) [254]. Therefore, the contribution of the equation of state would be negligible [250]. The material constants for the material description have been taken from Nguyen et al. [253] and are summarised in Table 7.4. Additionally, nine plasticity constants are implemented to the model in order to define the anisotropic yield surface, whose expression is the following [242]: 


$$
\begin{aligned}
f\left(\sigma_{i j}\right) & =a_{11} \sigma_{11}^{2}+a_{22} \sigma_{22}^{2}+a_{33} \sigma_{33}^{2}+2 a_{12} \sigma_{11} \sigma_{22}+2 a_{23} \sigma_{22} \sigma_{33}+ \\
& +2 a_{13} \sigma_{33} \sigma_{11}+2 a_{44} \sigma_{23}^{2}+2 a_{55} \sigma_{31}^{2}+2 a_{66} \sigma_{12}^{2}
\end{aligned}
$$

On the other hand, delamination is modeled as a surface-cohesive interaction where surface debonding is controlled by means of a mixed mode criterion involving failure modes I and II [254]. The parameters defining delamination are the Mode I normal strength $(1.2 \mathrm{MPa})$ and fracture energy $\left(0.544 \mathrm{Nmm}^{-1}\right)$ and Mode II shear strength $(1.8 \mathrm{MPa})$ and fracture energy $\left(1.088 \mathrm{Nmm}^{-1}\right)$. These constants were experimentally obtained from quasi-static tests in the literature and posteriorly Hazzard et al. [254] adjusted the value of the Mode II shear strength to $2.6 \mathrm{MPa}$ in order to achieve a better match with the experimental impact tests at ballistic velocities. This last value is therefore assumed for further simulations in this section.

\begin{tabular}{cccccc}
\hline \multicolumn{7}{c}{ Elastic properties } \\
\hline$E_{11}(\mathrm{MPa})$ & $E_{22}(\mathrm{MPa})$ & $E_{33}(\mathrm{MPa})$ & $\nu_{12}$ & $\nu_{13}$ & $\nu_{23}$ \\
\hline 26900 & 26900 & 3620 & 0 & 0.1 & 0.5 \\
& $G_{12}(\mathrm{MPa})$ & $G_{13}(\mathrm{MPa})$ & $G_{23}(\mathrm{MPa})$ \\
\hline \multicolumn{7}{c}{42.3} & 30.7 & 30.7 \\
\hline \multicolumn{6}{c}{} \\
\hline \multicolumn{7}{c}{ Strength properties } \\
\hline$X_{11}(\mathrm{MPa})$ & $X_{22}(\mathrm{MPa})$ & $X_{33}(\mathrm{MPa})$ & $S_{12}(\mathrm{MPa})$ & $S_{13}(\mathrm{MPa})$ & $S_{23}(\mathrm{MPa})$ \\
\hline 753 & 753 & 1.07 & 35.2 & $\infty$ & $\infty$ \\
\hline
\end{tabular}

Table 7.4: Summary of the mechanical properties of the UHMWPE composite extracted from the work by Nguyen et al [253]. 


\subsection{Comparison of the protective capacity}

Results obtained from the ballistic impact simulation on the ten shells are presented in this section. In order to establish a proper comparison between them, results will be first discussed separatedly for each material group (aramid-only, UHMWPE-only and hybrid) and then altogether in order to draw general conclusions.

\subsubsection{Aramid configurations}

The first group studied is conformed by integral woven-aramid configurations, which consist of 16 plies modeled with solid elements accounting for a total thickness of the helmet shell of $8 \mathrm{~mm}$. The only difference between them is the orientation of each ply: in \#A1, the plies are all oriented at $0^{\circ} / 90^{\circ}$ while in \#A2 the aramid plies alternate between $0^{\circ} / 90^{\circ}$ and $45^{\circ} /-45^{\circ}$.

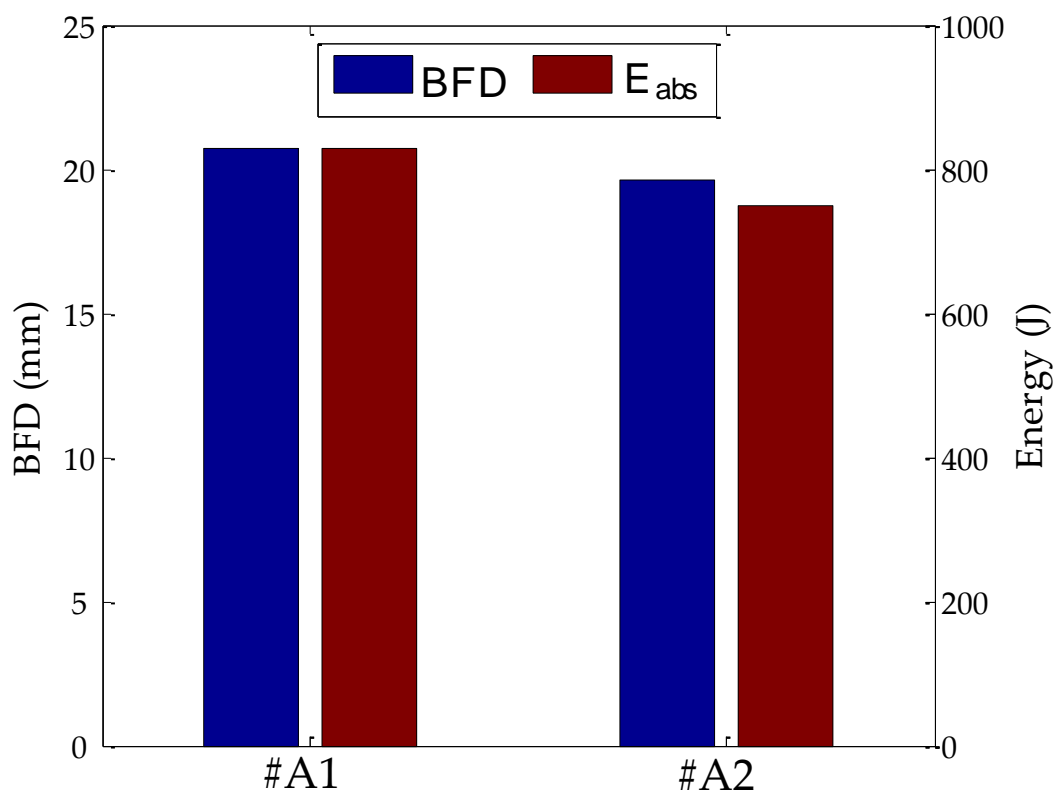

Figure 7.3: Maximum backface deformation and energy absorbed by the two aramid-fabric configurations.

Fig. 7.3 represents the simulated maximum values of the backface deformation and the energy absorbed by the shell for each case. The orientation of 
the plies has a visible effect on both parameters, being more pronounced for $E_{a b s}$, which is a $10 \%$ higher $(830 \mathrm{~J})$ for \#A1 than \#A2 $(750 \mathrm{~J})$. This result would imply a better performance of the first in terms of impact attenuation (ballistic resistance). The penalty for this increased energy absorption is the increase in $\mathrm{BFD}$, of about a $5 \%$ with respect to \#A2.

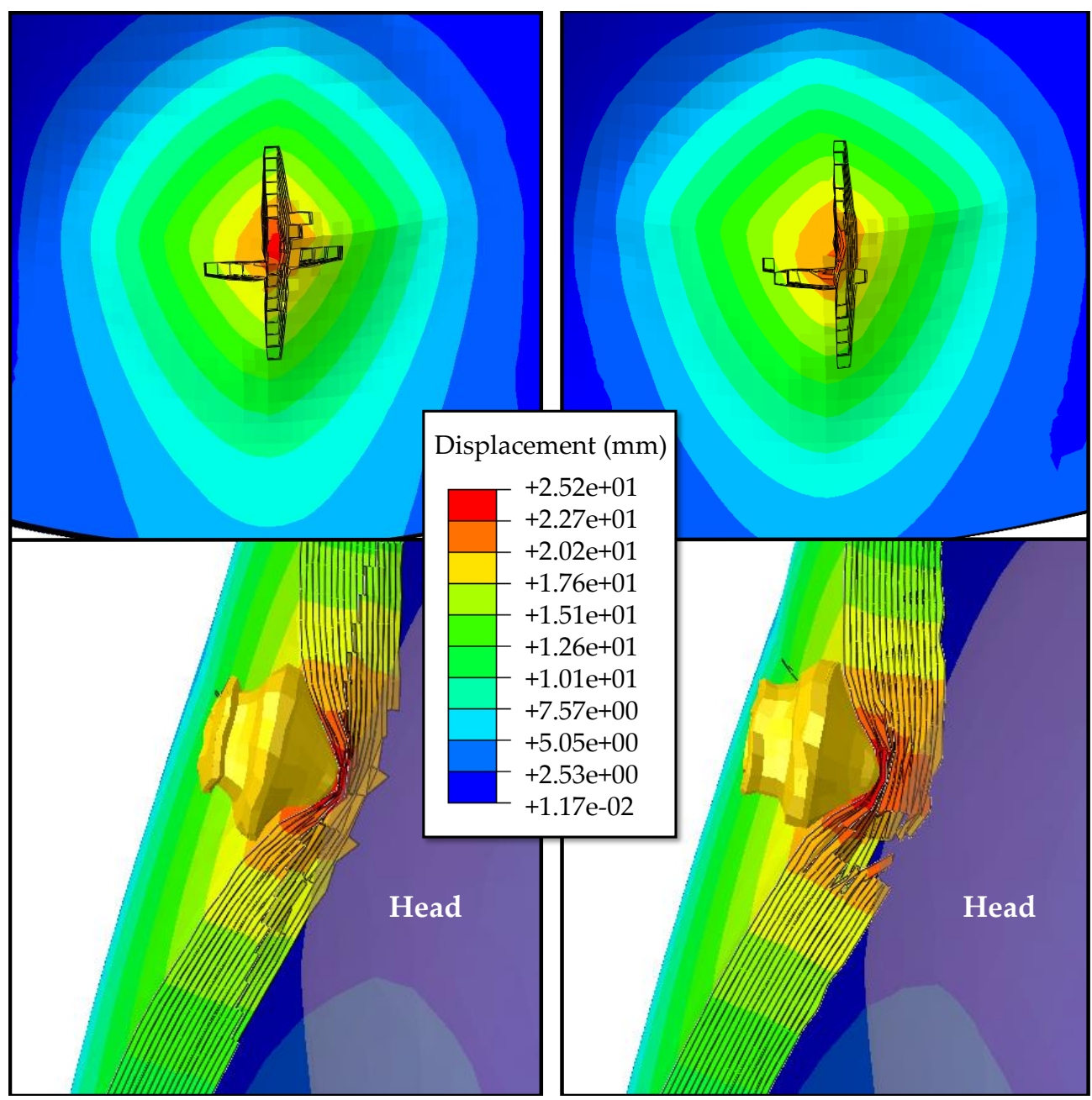

Figure 7.4: First row: displacement contours of the inner impacted region of the helmet shell for \#A1 (left) and \#A2 (right). Second row: cross-section of the deformed shell for \#A1 (left) and \#A2 (right). 
The predominant mechanism that enhances energy absorption is the fiber tensile failure, which is triggered by the bending moment resultant from the impact, and reaches its maximum at the inner layer of the shell. Therefore, fiber breaking starts (in the through-thickness direction) from the deepest layer towards the striking surface. In this surface, elements fail due to compressive loading on the concave region created by the bullet before its rebound.

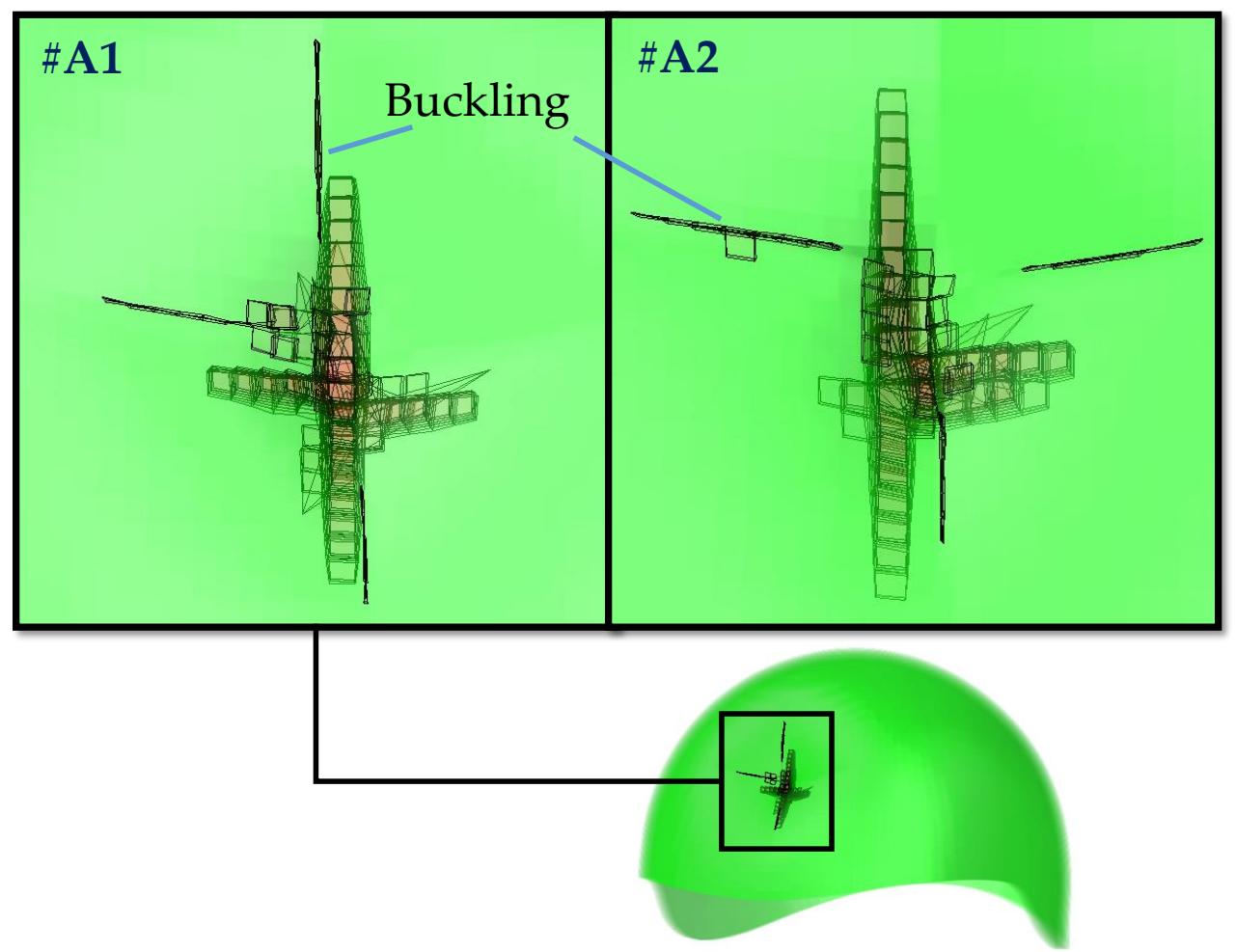

Figure 7.5: Frontal view of the failed elements after the impact. The most clear-cut elements correspond to the exterior surface, while increased translucency means location at the deeper layers.

Damage sustained by the helmet shell at the moment of maximum BFD for each configuration is illustrated in Figs. 7.4 and 7.5. The top row of Fig. 7.4 depicts the displacement contours of the helmet shell at the inner surface. Displacement values are taken as positive in the direction of the projectile. Two main differences can be appreciated between the contours obtained from 
case \#A1 (left) and \#A2 (right). The first is the peak displacement values achieved, which are 2.52 and $2.33 \mathrm{~mm}$, respectively, which in both cases are reached at the contact region between the bullet and the shell surface. Secondly, paying attention to the contour shaping, it can be appreciated that the deformed region in \#A1 is larger in the top-bottom direction (coincident with the head's sagittal plane) while this effect is less severe in \#A2 and contrasted by a widening in the perpendicular direction. It can be inferred that the fact that BFD in \#A2 approaches a more circular-like shape is due to the quasi-isotropy of the material achieved through the alternation of fabric orientations. This attenuates failure concentration on a single fiber direction.

The above discussion can be seen in the bottom row of Fig. 7.4. In these two images (left for case \#A1 and right for \#A2) a transversal cut of the helmet at the instant of maximum BFD is shown. The purple region placed on the right side of each picture represents the external surface of the head model on its undeformed state. The intersection between the deformed helmet shell and the head surface represents the overpass of the stand-off distance. As can be appreciated from the pictures, this penetration into the security zone is more pronounced for configuration \#A1, as the majority of the shell layers have failed at the impact site and only four of them are defeating the advance of the bullet. In the case of \# $\mathrm{A} 2$, there are more undamaged and partially-damaged layers in the through thickness direction to withstand the remaining energy of the bullet.

Fig.7.5 illustrates the total number of failed elements (from a frontal point of view) for each shell after the impact. the shell latice is represented as a translucent green volume in order to distinguish the depth of the failed elements: the most blurred and uncoloured ones are placed at the inner shell surface while the most clear-cut and coloured (salmon) are located close to the striking surface. For configuration \#A1, there are failed elements through almost all the thickness in the vertical direction (coincident with the fibers oriented at $0^{\circ}$ ) and also a relevant number in the perpendicular direction. This cross-shaped failure pattern is expected from an orthotropic material such as \#A1. The evaluation of case \#A2 is not straightforward: most of the failed elements are placed in the vertical direction, with a total length superior to the obtained in \#A1. Nonetheless, these are located in deeper layers of the shell, and no significant failure is found in the proximities of the striking surface. Failure is additionally reached in a certain area in the $90^{\circ}$ direction, but it does not follow a symmetrical pattern. 


\subsubsection{UHMWPE configurations}

In the case of the UHMWPE-based helmet shells, the influence of the stacking sequence is remarkable. As a reminder, configurations \#P1, \#P2 and \#P3 are conformed by 36 layers (with a total shell thickness of $8.64 \mathrm{~mm}$ ) and they differ in the stacking sequence. On the other hand \#P4 comprises the same layup as \#P3 but accounts for 48 layers (which involves a final thickness of $11.52 \mathrm{~mm})$.

Maximum recorded BFD values and the energy absorbed by each configuration are schematised in Fig.7.6. In case \#P1, with a purely helicoidal orientation sequence, the low values reached for both performance parameters is due to a rapid perforation of the bullet. Fig.7.7 represents a detailed view of the through-thickness failure process until penetration. This result is coherent as Karthikeyan et al. [241] achieved perforation on a panel with a similar layup and projectile at much lower velocities $\left(174 \mathrm{~ms}^{-1}\right)$.

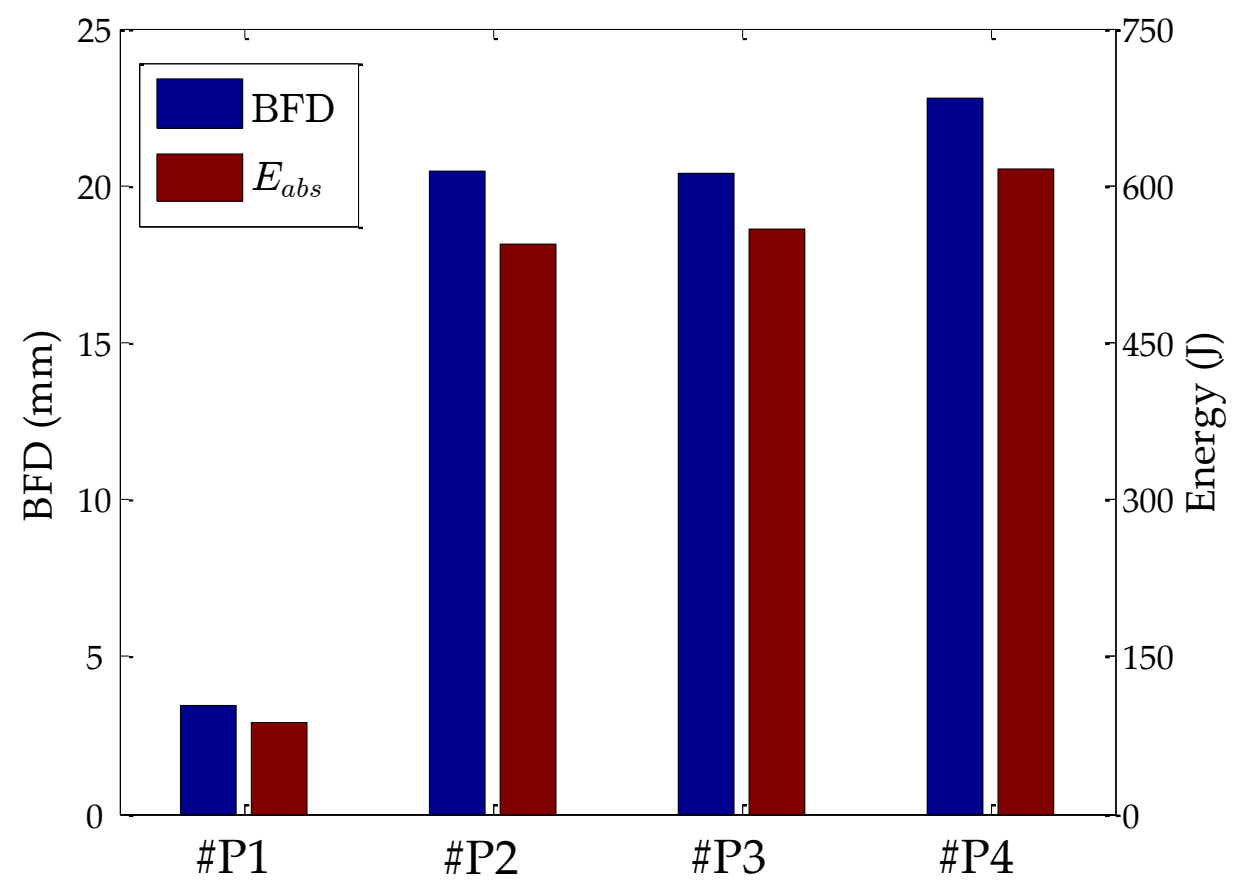

Figure 7.6: Backface deformation and energy absorbed by each of the UHMWPE-based helmet shells. 


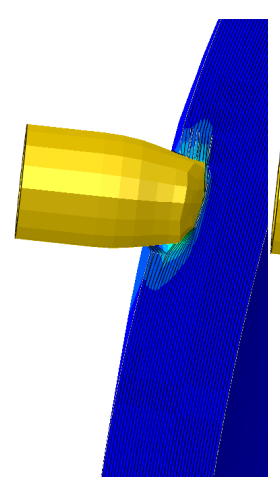

$10 \mu \mathrm{s}$

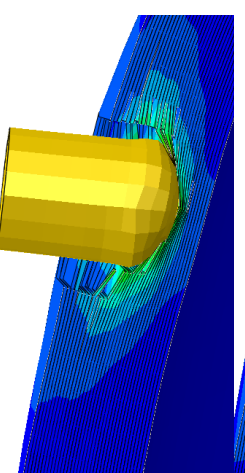

$20 \mu \mathrm{s}$

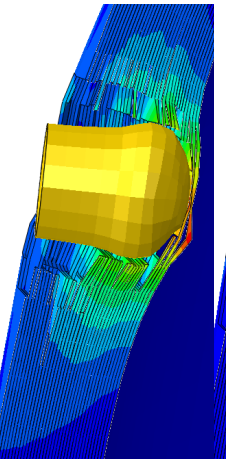

$30 \mu \mathrm{s}$

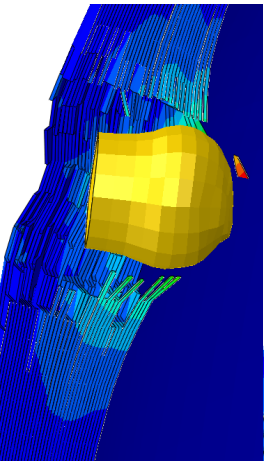

$40 \mu \mathrm{s}$

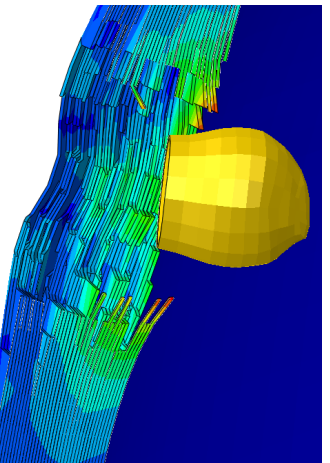

$50 \mu \mathrm{s}$

Figure 7.7: Time sequence of the $9 \mathrm{~mm}$ bullet impacting the \#P1 shell.

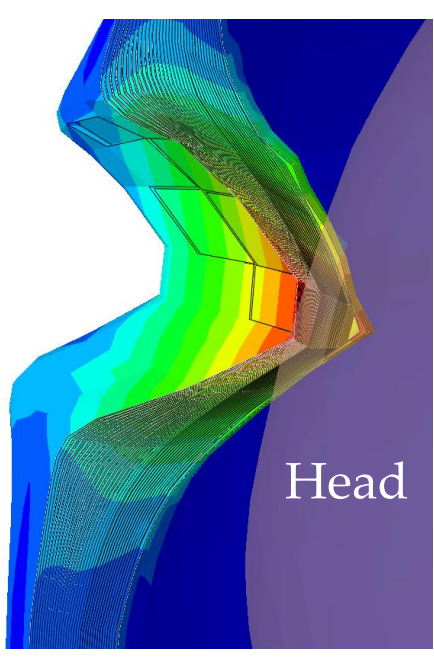

\#P2

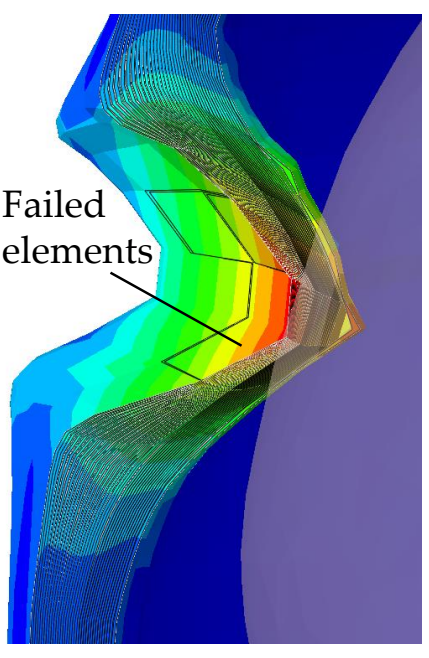

\#P3

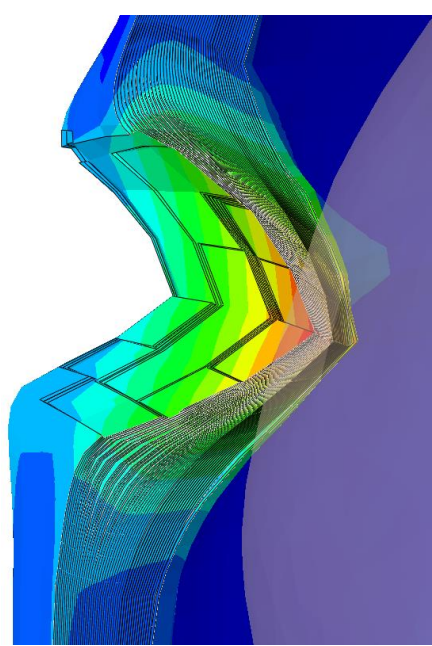

\#P4

Figure 7.8: Deformation of shells \#P2, \#P3 and \#P4 at the region impacted by the bullet.

The remaining three, which account for an orthotropic behaviour at the layer level, present results in the same order of magnitude for both BFD and $E_{a b s}$. In these cases, even though the helmet shell is not perforated, the resulting deformation in the impact region penetrates the skull bone in a 
highly localised area. This phenomenon is illustrated in Fig. 7.8, in which the translucid surface represents the contour of the head model at its initial state. The overlap between this surface and the deformed helmet shell represents the final perforation in the head.

Focusing in cases \#P2 and \#P3, which differ in the ply orientations, no substantial difference in either $E_{a b s}$ or BFD is achieved. In previous works from the literature it was hypothesised that while keeping constant orientation of the layers along the entire lay-up increases the energy absorption, alternating the angles between layers enhances the reduction of the maximum BFD, as the shell tends to behave in a quasi-isotropic manner. In the results presented in this study, the first hypothesis is accomplished, whereas the second reveals an opposite trend. The reason for this result may be due to several factors, such as the shell curvature, the angle step between plies (that has been taken as $15^{\circ}$ ), and the employment of a different ammunition type.

Increasing the thickness of the UHMWPE shell on a 33\% (\#P4) leads to an approximatedly $10 \%$ increase in the amount of energy absorbed with respect to \#P2 and \#P3, reaching a peak level closer to the aramid configurations. Nonetheless, the associated BFD value increases in the same proportion, reaching values beyond the stand-off distance.

These results suggest that the addition of composite layers (and therefore the increase in thickness) will have a positive effect in resistance to penetration but does not represent a solution for the issue of BHBT. Furthermore, the 48layer configuration does lead to an increase in the shell mass $(1.2 \mathrm{~kg})$ with respect to the aramid configurations $(1.03 \mathrm{~kg})$ and therefore the whole point of employing UHMWPE, which is weight reduction, would be lost.

\subsubsection{Hybrid configurations: Aramid and Carbon Fiber}

In a previous subsection the performance of the two aramid-only helmet shells modelled in this study was discussed. In the current section, the purpose is to discuss the benefits of hybridisation, in this case, of the same aramid-fabric employed por \#A1 and \#A2 with a reinforcement of two carbon fiber-reinforced plastic (CFRP) layers. Hybridisation has as an objective to combine the best qualities of different materials in order to achieve a specific improvement in the performance of a system. 
One of the results obtained at the first part of this study suggested that the aramid-only configuration \#A2, with alternating orientations between adjacent plies, offered an adequate protection level in terms of BHBT avoidance. For this reason, in this part of the study the focus will be set on improving the \#A1 shell for blunt trauma prevention by adding a reinforcement of CFRP. This reinforcement, consisting of two layers of woven carbon fibre, will be placed either at the striking surface of the shell (\#HCA) or the inner surface (\#HAC).

After submitting these hybrid shells to the same ballistic analysis as the previous cases, the backface deformation value and the energy absorbed have been computed and schematised in Fig.7.9. For an easier comparison, the values that were recorded from \#A1 have been included in the plot.

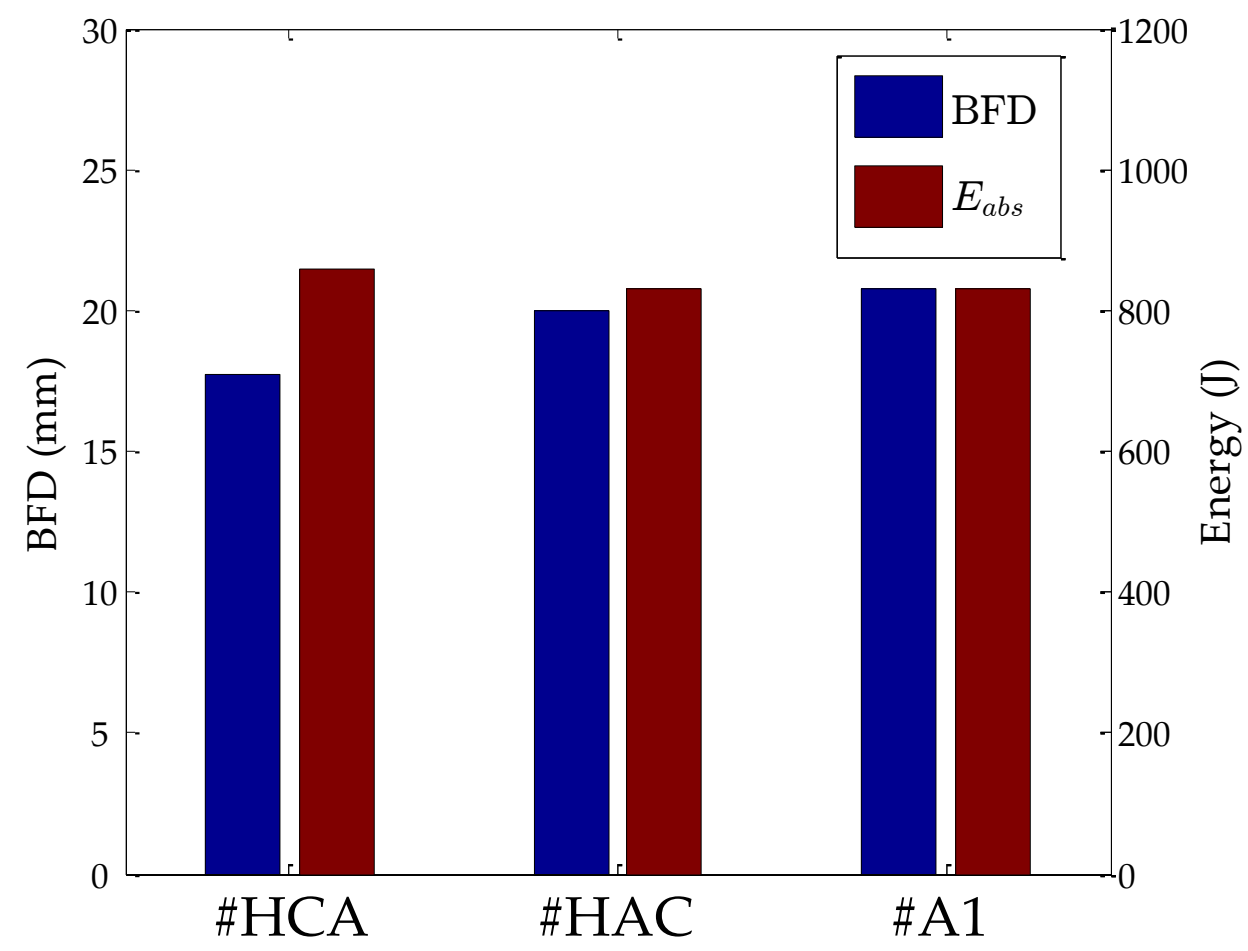

Figure 7.9: Backface deformation and energy absorbed by each of the carbonaramid hybrid shells and the aramid-only configuration \#A1. 
It is noticeable that the addition of the carbon layers have dissimilar effects depending on their through-thickness position. In both hybrid configurations the resulting BFD is lower than the aramid-only (\#A1), which means an improvement in blunt trauma prevention, but in the case of the energy absorption, the tendency is not so straightforward. In fact, despite increasing the total shell thickness by $0.8 \mathrm{~mm}$ with respect to \#A1, configuration \#HAC leads to a slightly lower value of the absorbed energy than the aramid-only shell. On the contrary, configuration \#HCA leads to an increase in $E_{a b s}$ of about $3.6 \%$ while reducing the maximum BFD in a $17.1 \%$.

The relation between the relative position of the carbon reinforcement with respect to the aramid stack and the amount of energy absorbed obtained in this study is in agreement with previous published results on experimental (Hazell and Appleby-Thomas (2009) [248]) and numerical (Bandaru et al. (2015) [249]) works on hybrid Kevlar-CFRP panels. In Hazell and ApplebyThomas [248], ballistic tests are carried out at maximal projectile velocities of $352 \mathrm{~ms}^{-1}$ and the baseline targets are $5.3 \mathrm{~mm}$ thick CFRP panels with Kevlar reinforcements of $0.7 \mathrm{~mm}$. Although the proportion of materials is the opposed to the one assumed in this section, in which aramid is predominant compared to CFRP, [248] did find that placing the Kevlar after the main CFRP panel increased the total absorbed energy by a $100 \%$ with respect to the opposite layup. Bandaru et al. [249] simulated ballistic impacts with geometries and boundary conditions closer to the present study: $7.2 \mathrm{~mm}$ panels with 4 carbon layers and 12 of Kevlar, impacted at a velocity of $400 \mathrm{~ms}^{-1}$ with a $7.45 \mathrm{~g}$ projectile. The same trend found in [248] was obtained but the difference in $E_{a b s}$ was not as pronounced (12-25\%). In the present study, this difference (between \#HCA and \#HAC) is $3.6 \%$, a value that is consistent with [249] given the reduced proportion of CFRP with respect to aramid assumed here.

Nonetheless, the overall behaviour of the shell structure is prone to be influenced by its geometry, which involves sharp changes in curvature, and it is reasonable to think that its ballistic response may differ in some aspects from the one achieved with composite planar plates. 


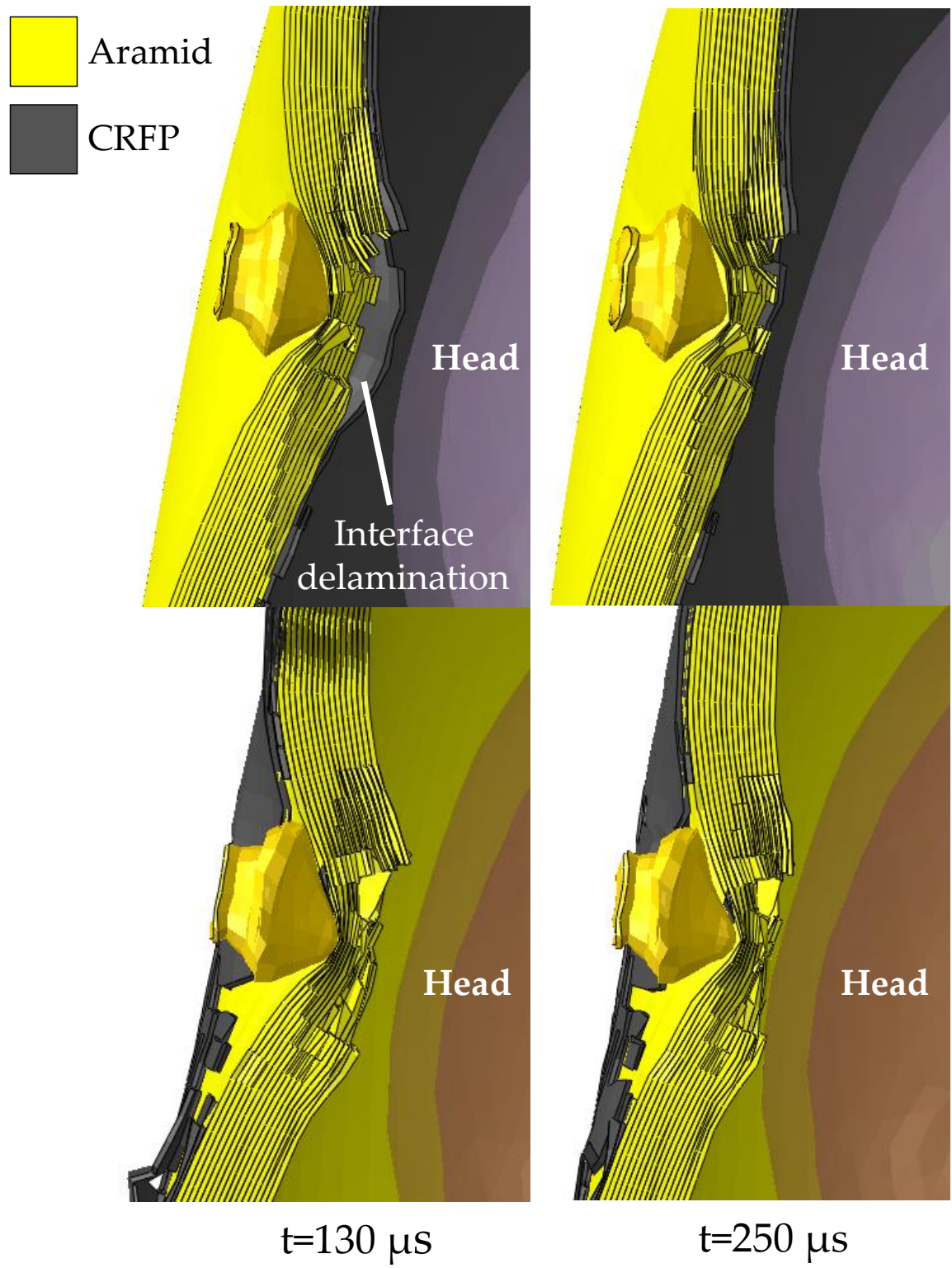

Figure 7.10: Transversal cut of the helmet shell at two different times from the impact simulations on shells \#HAC (top) and \#HCA (bottom).

The reduction of the maximum BFD value is achieved in both hybrid 
configurations with respect to \#A1. A cross-section cut of the impact process for each hybrid shell is illustrated in Fig.7.10. When the CFRP reinforcement is placed at the striking surface (\#HCA), this material absorbs part of the initial bullet kinetic energy and fiber failure is reached not only in the contact area but also in regions remote from the impact point. Therefore, the kinetic energy reaching the aramid stack is attenuated and the subsequent damage caused by fiber tensile failure in the inner surface becomes less relevant than it was without the carbon addition. In fact, it was seen in Fig. 7.4 that the failed region in the \#A1 shell was concentrated along the sagittal direction, and this localised loss of stiffness caused an increased BFD value in this region. Nonetheless, in \#HCA, the total surface subjected to failure is reduced and evenly distributed along all directions, resulting in a BFD decrease of $17.1 \%$.

This last finding is, however, not fully accomplished for the opposite position of the CFRP stack (\#HAC). This reinforcement does in fact contribute to reduce the maximal BFD (with respect to \#A1) and, as can be appreciated in Fig. 7.10 (right column) the maximum displacement of the inner surface at the time of maximal advance of the bullet $(t=250 \mu \mathrm{s})$ is even less than the reached by \#HCA. In this later, the cause of increase in the BFD value is the transient phenomenom of delamination of the carbon layers from the aramid, which takes place at about $100 \mu s$ after the impact. The detached area of CFRP strikes the foam padding towards the head, reaching in this point the maximum $B F D$ value.

In view of the results obtained, the use of CFRP as a reinforcement to the aramid shell \#A1 offers benefits such as an increase in energy absorption and BFD reduction when it is placed on the striking surface of the helmet.

\subsubsection{Hybrid configurations: UHWMPE and aramid}

As discussed in the section dealing with integral UHMWPE shells, the energy absorption of these is considerably lower than the aramid-only. Even though being capable of preventing bullet penetration, the plastic deformation left behind the projectile leads to large values of $\mathrm{BFD}$, causing penetrating injury in the head.

Aramid fabric has been chosen to compensate the highly deformable behaviour of UHMWPE. Maximum BFD is intended to be controlled by placing 9 woven-aramid plies at the striking (\#HAP) or the inner (\#HPA) shell surface. These hybrid configurations both have the same total number of layers 
and orientations as \#P3, and the aramid plies substitute the polyethylene plies instead of being added to the thickness. For this reason, the results of hybridisation will be compared to the non-hybrid \#P3.

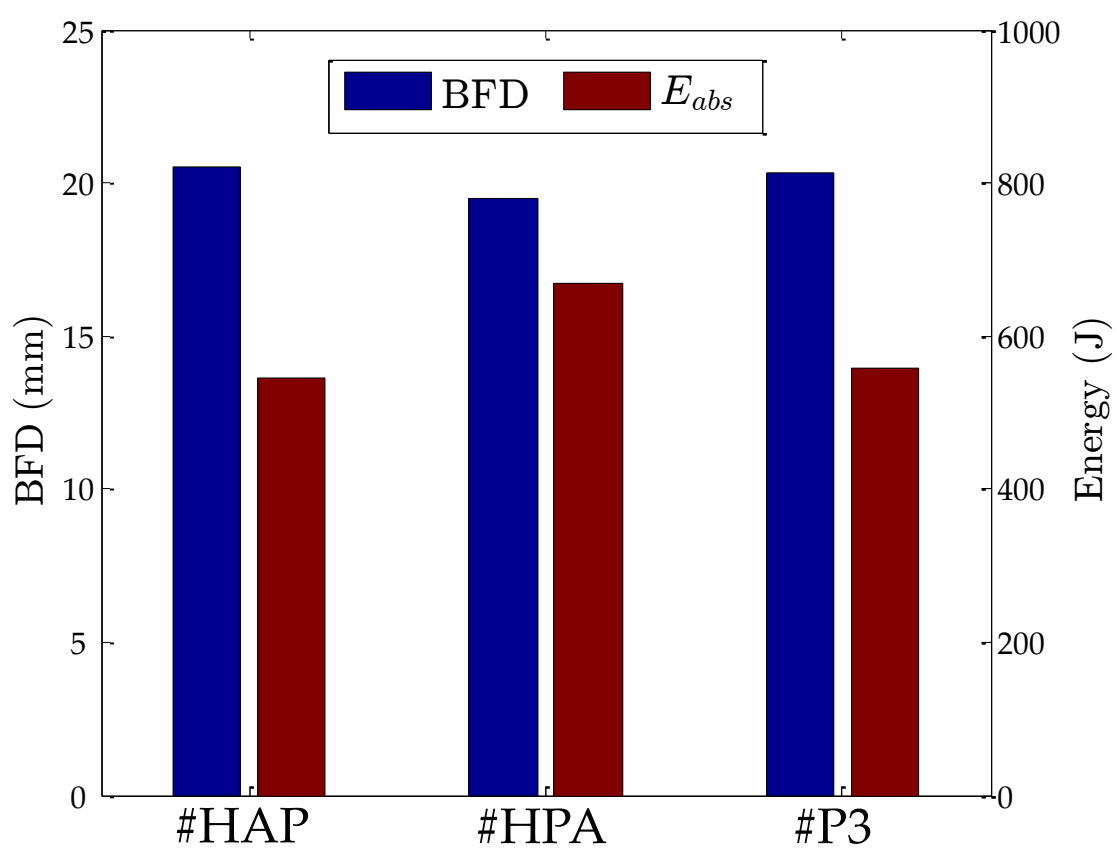

Figure 7.11: Maximum backface deformation and energy absorbed by the hybrid UHMWPE-aramid configurations and the UHMWPE-only shell.

Fig.7.11 schematises the maximum reached BFD and the energy absorbed by the hybrid and non-hybrid UHMWPE-based shells. It can be easily seen that case \#HAP, with the aramid stack placed on the striking surface, does not provide any improvement with respect to \#P3 in terms of both variables. In fact, $E_{a b s}$ is slightly lower and the maximum BFD is $0.2 \mathrm{~mm}$ greater. Nonetheless, when the aramid stack is placed at the inner part, there is a $20 \%$ increase in the energy absorbed and a $4 \%$ decrease in the maximum deformation. Thus, this stack configuration benefits both ballistic resistance and blunt trauma prevention. 


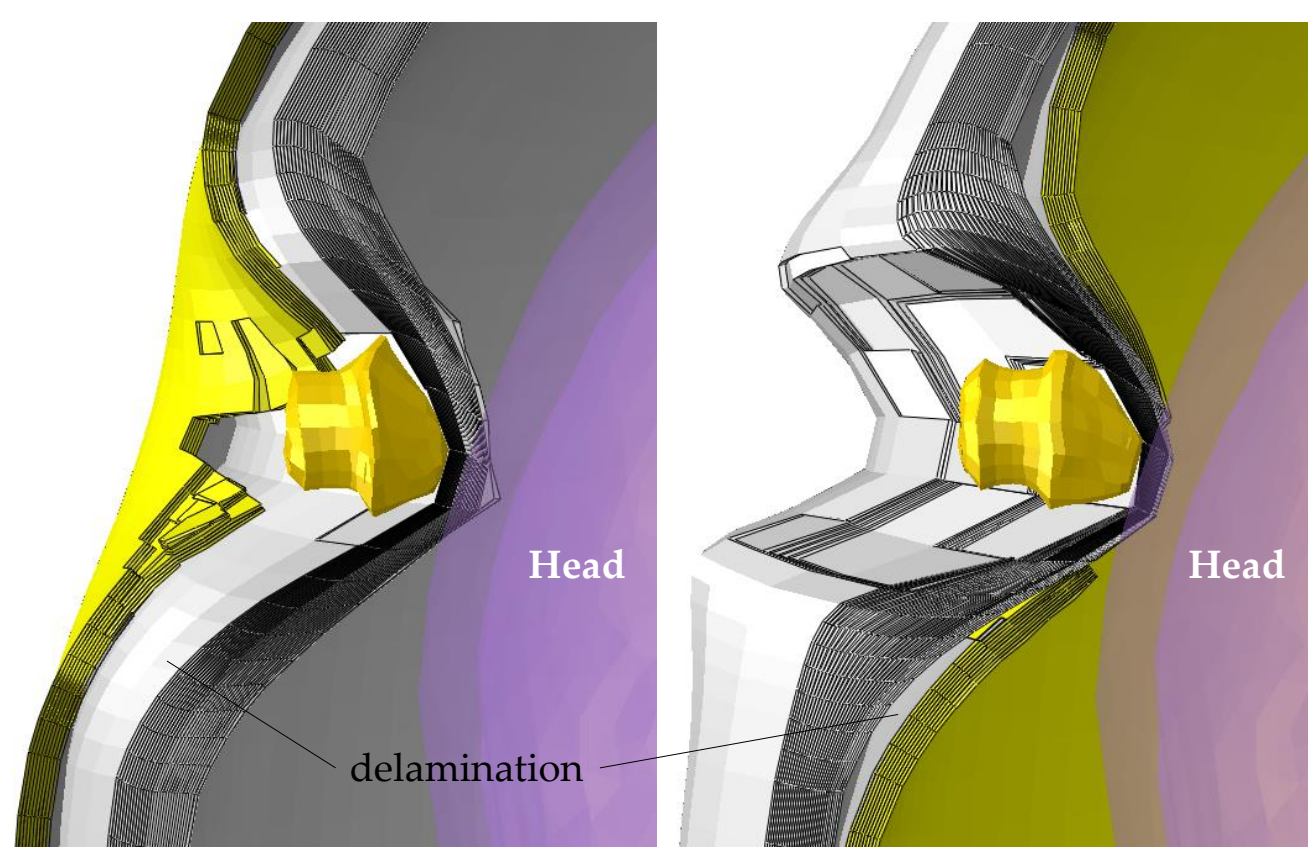

Figure 7.12: Cross section of the deformed shells \#HAP (left) and \#HPA (right).

Fig. 7.12 illustrates a transversal cut of the helmet shell at the moment of maximum BFD. The deformation and damage patterns along the thickness change completely by placing the aramid stack at one location or another. When it is set at the striking surface (\#HAP), the aramid layers absorb part of the initial energy of the impact until their complete failure. When the aramid stack is perforated, the bullet keeps advancing towards the UHMWPE plies. Given the great difference in matrix strengths, a sudden delamination takes places at the interface between the two composites. The detachment occurs at the impact site and along the mid-sagittal plane, as can be appreciated in Fig. 7.13 (left), coinciding with the fabric orientations. Therefore, the UHMWPE stack in contact with the bullet has to deal with the remaining kinetic energy and achieves a large piramidal deformation until penetrating the skull (Fig. 7.12 (left)).

On the other hand, the response in the \#HPA shell is nearly the opposite. Polyethylene being the first material in contact with the bullet, its subsequent deformation is constrained by the presence of the stiffer aramid layers in the 
inner shell. As a consequence, the UHMWPE region surrounding the impact site tends to deform outwards from the surface, in the opposite direction to the projectile (Fig. 7.13 (right)). Simultaneously, extensive fiber failure in the impact site is obtained. This response is acompanied by the local delamination between the most external polyethylene layers, as can be observed in Fig. 7.12 (right). As the interlaminar strength of the aramid stack is greater than for the UHMWPE, local failure at the interface is reached. Nonetheless, unlike the \#HAP case, the two materials remain in direct contact after the delamination, which enhances additional energy dissipation due to the friction between both surfaces. Furthermore, the damaged aramid layers contribute to limit the magnitude and distribution of the deformation behind the projectile. In fact, the BFD region acquires an almost perfectly circular shape. In sum, \#HPA provides a better overall performance than \#P3 despite the $120 \mathrm{~g}$ increase in shell mass.

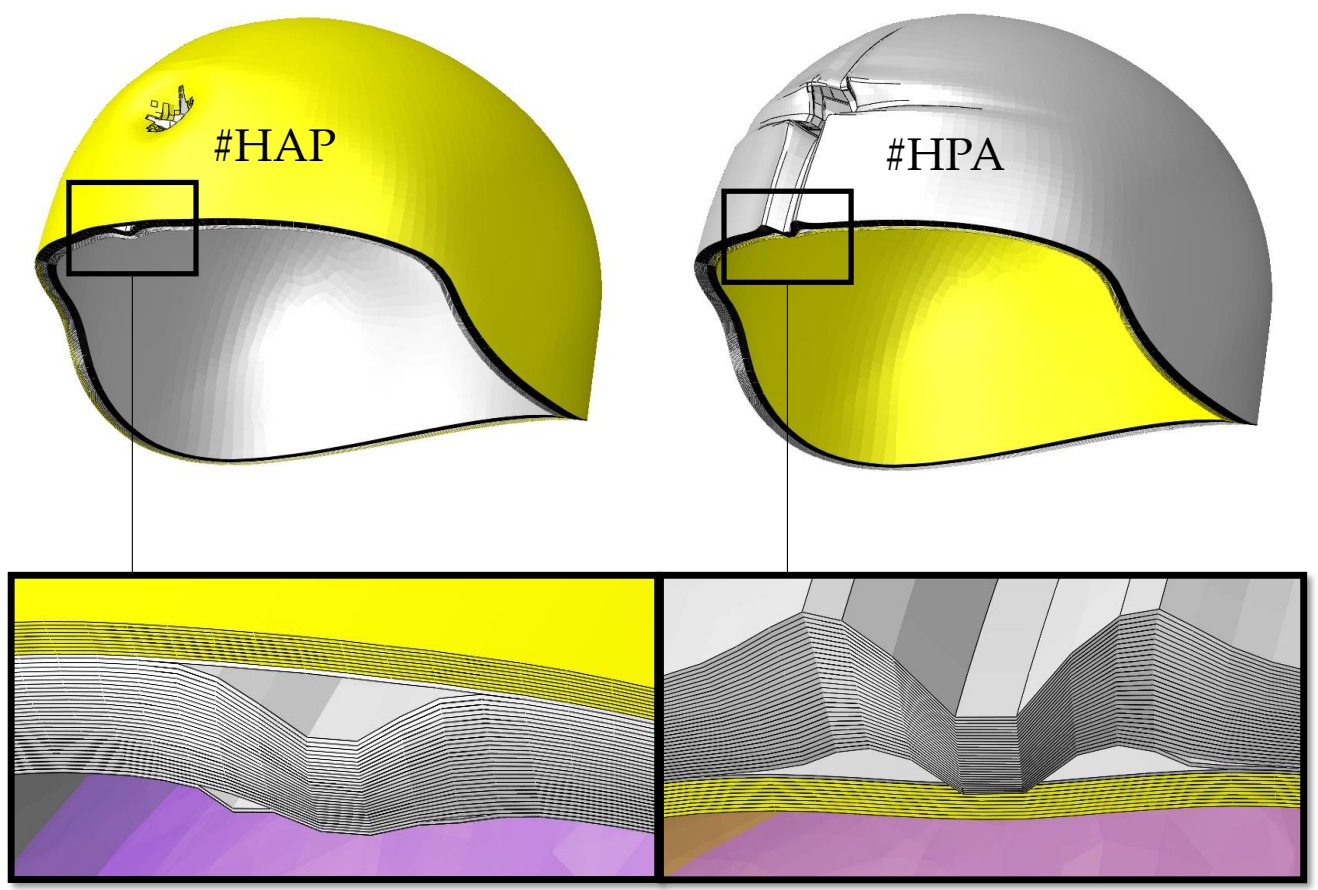

Figure 7.13: Delamination during impact in the \#HAP (left) and \#HPA (right) shells. 


\subsection{Associated head trauma}

After discussing the performance of the different helmet shells simulated against ballistic impact, the aftermaths on the head model have been analysed and are summarised in Table 7.5. Most of the studied cases led either to bullet perforation or the head was penetrated by the BFD itself.

\begin{tabular}{c|c|c} 
Material & Name & Injury outcome \\
\hline Aramid & \#A1 & Basilar skull fracture \\
& \#A2 & No observable damage \\
\hline Polyethylene & \#P1 & Cranium perforated by the bullet \\
& \#P2 & Cranium perforated by BFD \\
& \#P3 & Cranium perforated by BFD \\
& \#P4 & Cranium perforated by BFD \\
\hline Aramid + CFRP & \#HCA & No observable damage \\
& \#HAC & No observable damage \\
\hline Aramid + Polyethylene & \#HAP & Cranium perforated by BFD \\
& \#HPA & Cranium perforated by BFD \\
\hline
\end{tabular}

Table 7.5: Head injury outcome from the different helmet shell configurations simulated.

Aramid-only configurations prevent penetrating fractures but a frontobasal skull fracture arises when employing the \#A1 shell, which kept constant orientations. In all the polyethylene-based cconfigurations skull perforation was achieved, being caused in all cases by the excessive deformation of the inner shell layers except for \#P1 where bullet penetration occurs. Fracture severity is the lowest in \#P4 due to the $33 \%$ increase in shell thickness with respect 
to its analogous. This finding suggests that the addition of more HB26 layers would shift the skull trauma from penetrating to blunt.

Hybridising leads in all cases to less-magnitude injuries, due to greater number of layers and therefore larger thicknesses, but only provide full protection in the cases where aramid is coupled with carbon fiber.

\subsubsection{Conclusions}

In this chapter, several helmet shell configurations, varying in both their material constituents and stacking sequence have been simulated against frontal ballistic impact. It has been explained that two main variables reveal the performance of a combat helmet: energy absorption and backface deformation. The first becomes especially relevant in order to determine the ballistic limit of the helmet, namely, the maximum projectile velocity it can withstand without complete perforation. Normally, the ballistic limit becomes more critical when dealing with small threats like fragments whereas the backface deformation is critical against greater-mass ammunitions. Hence, an optimal performance against multiple threats is a sort of tradeoff between variables. In this chapter the focus is set on the protection against $9 \mathrm{~mm}$ caliber bullets, and therefore BFD is considered as the most critical parameter.

Taking into account several factors (maximum BFD, shell thickness and mass, and, especially, resulting head injury), the best performance is attained from the aramid-only shell \#A2, in which the woven plies are stacked alternatedly at $0^{\circ} / 90^{\circ}$ and $45^{\circ} /-45^{\circ}$. However, this alternating sequence presents the drawback of reducing the energy absorbed with respect to a shell maintaining same orientations throughout the thickness (case \#A1). In order to reduce the magnitude of the blunt trauma achieved in \#A1 but without compromising its energy performance, two hybrid aramid-carbon fiber configurations have been analysed. These consisted of the addition of two woven CFRP layers at either the striking or inner helmet surfaces. Both cases led to an improved performance of \#A1 in terms of $E_{a b s}$ and BFD, being this benefit more relevant in the case \#HCA. In summary, with an increase of $120 \mathrm{~g}$ in mass from the \#A2 configuration, an equivalent level of protection is achieved while boosting the energy absorption by more than a $10 \%$.

UHMWPE-based configurations, despite providing a desirable weight reduction, did not reach the performance levels of the aramid and aramid-carbon 
shells. Their main drawback is their high-deformation capacity, greatly surpassing the stand-off distance and causing the BFD to penetrate the head. If hybridised with aramid woven layers, specially when these are placed in the inner shell surface, maximum BFD decreases slightly and $E_{a b s}$ gets closer to the aramid configurations. Nonetheless, for this improved performance a considerable mass addition is required and therefore the benefits of employing a lightweight material are overshadowed. 



\section{Chapter 8}

\section{Numerical modeling of a human head with further anatomical details}

Chapters 6 and 7 of this Thesis aimed to predict the injury potential of different ballistic impacts on a head protected with a helmet. The head model employed for this purpose was sufficient to capture the injury mechanisms addressed in these chapters, such as skull fracture or accelerations at the center of gravity. The strain and pressure-based criteria assumed for the prediction of DAI and contusions, respectively, were the CSDM and DDM. Both of them are cumulative measures which are intended for an homogeneous brain tissue and therefore suitable for a head model with the detail level presented in Chapter 5 .

Nonetheless, the geometrical and structural complexity of the CNS and other intracranial contents has been repeatedly highlighted along this Thesis. If a deep study of specific injury mechanisms is desired, further subdivision of the tissues in the numerical head model should be performed. For instance, if the purpose is to assess local axonal failure, the anisotropic reinforcement of neurons inside the brain lattice should be taken into account. Nonetheless, this sort of analysis would involve a complexity and computational expense that may be unnecessary depending on the problem to be studied.

The previously employed head model presented a reasonable computational expense and offered an adequate accuracy for the studies performed with it, 
Chapter 8. Numerical modeling of a human head with further anatomical

as they simulated impact conditions similar to the ones employed for its validation. Nonetheless, the omission of certain structures such as the meninges could lead to a non-accurate response on lateral or occipital impact situations. Furthermore, the assumption of homogeneous mechanical properties in the brain tissue does not enable the study of some specific stress concentrations that may occur when the head is subjected to rotational loads.

In order to enhance the study of various injury environments that involve combined impact directions, a numerical head model with further anatomical details has been developed following the same methodology as for the previous one.

\subsection{Model description}

A finite element (FE) model of the human head has been developed from CT images of a real patient, provided by Hospital La Fe from Valencia. The head dimensions of the patient fall within the range of the 50th male percentile. The segmentation of the medical images and the subsequent meshing were performed by means of ScanIp $v 6$ from Simpleware.

A total of 33 masks were differenciated based on image grayscale thresholds and atlas of the intracranial tissues, comprising soft tissues, bones, and fluids. The model comprises the following soft parts: cerebellum, brainstem (divided in medulla oblongata, pons and midbrain), optic tracts, hypothalamus, pituitary gland, left and right thalamus, fornixes and hypoccampus, corpus callosum, white and gray matter and dura matter. The bony structures included are the compact bone and diplöe for the skull, and the facial bone and upper jaw.

- Cerebellum

- Brainstem $\left\{\begin{array}{l}\text { Medulla Oblongata } \\ \text { Pons } \\ \text { Midbrain }\end{array}\right.$

- Left and right optic tracts

- Hypothalamus 
- Pituitary

- Left and right thalamus

- Left and right fornixes

- Left and right hypoccampus

- Corpus callosum

- White matter

- Gray matter

- Ventricles $\left\{\begin{array}{l}\text { Left and right ventricles } \\ \text { Third ventricle }\end{array}\right.$

- Sinuses $\left\{\begin{array}{l}\text { Superior sagittal sinus } \\ \text { Inferior sagittal sinus } \\ \text { Straight sinus } \\ \text { Left and right transverse sinuses }\end{array}\right.$

- Dura matter

- Bony tissues $\left\{\begin{array}{l}\text { Compact bone } \\ \text { Diplöe } \\ \text { Face and upper jaw }\end{array}\right.$

Additionally, the two internal membranes falx celebri and tentorium cerebelli were created and meshed using MATLAB and Python, ensuring that both cerebral hemispheres and the cerebellum remain separate leaving a gap to be filled with CSF. The converging edges of this membranes have been tied (with a nodal constraint) at the level of the straight sinus and the exterior edges have been attached to the dura matter, as both structures are indeed a prolongation of this later. All the described structures are illustrated in Fig. 8.1 and Fig. 8.2. In 8.1, both falx and tentorium are represented in translucent purple, revealing a mesh of four-node membrane elements (type M3D4R in Abaqus).

The image quality for the segmentation was not sufficient to distinguish all the internal brain structures, as computed tomography presents less resolution than, for instance, electromagnetic resonance. Therefore, some tissues like 
Chapter 8. Numerical modeling of a human head with further anatomical 200 details

bone and scalp could be separated by thresholding the grayscale whereas other parts were segmented based on both the medical images and well-established human atlas.

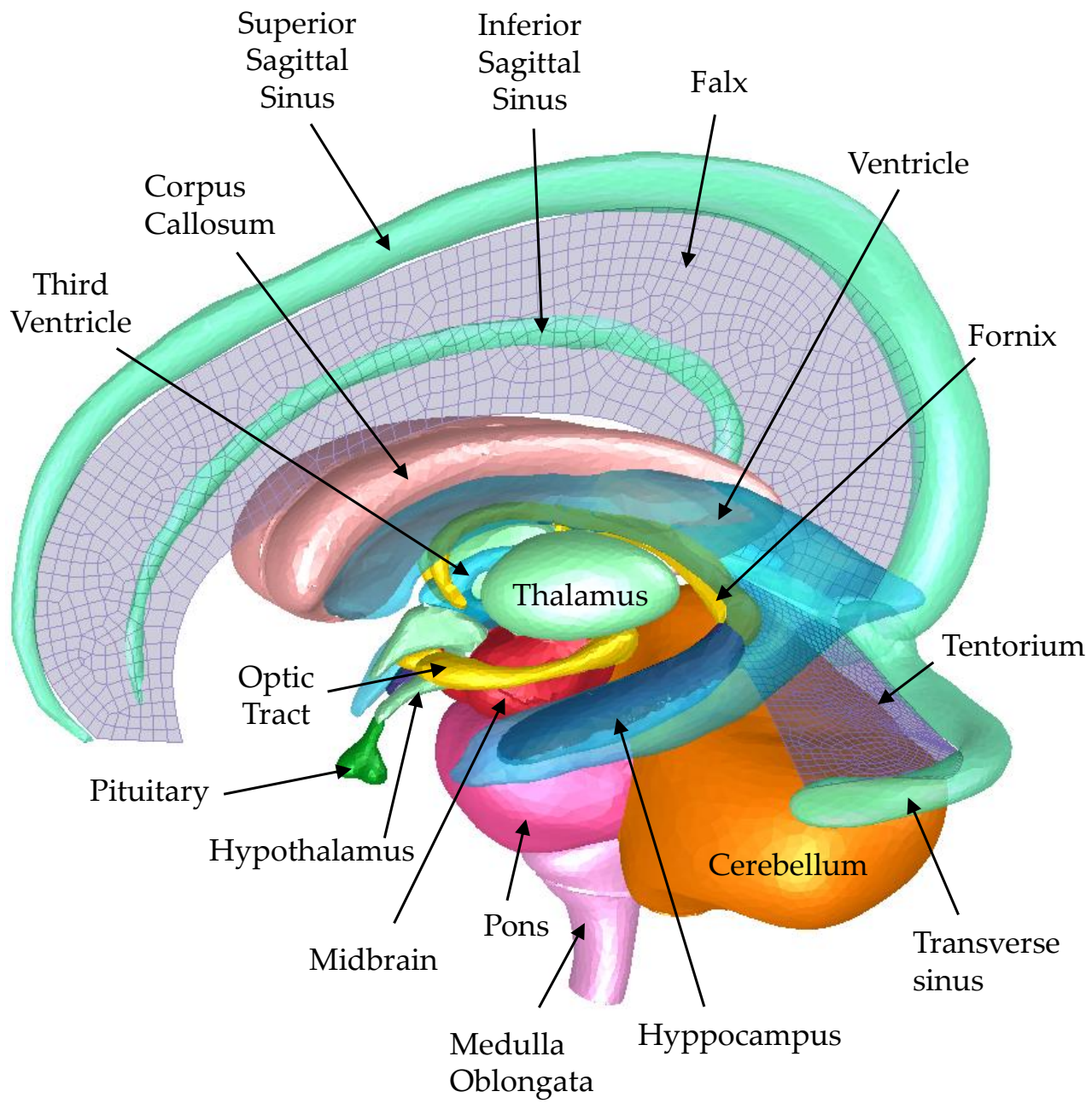

Figure 8.1: Some of the intracranial structures included in the FE head model presented in this Chapter. 

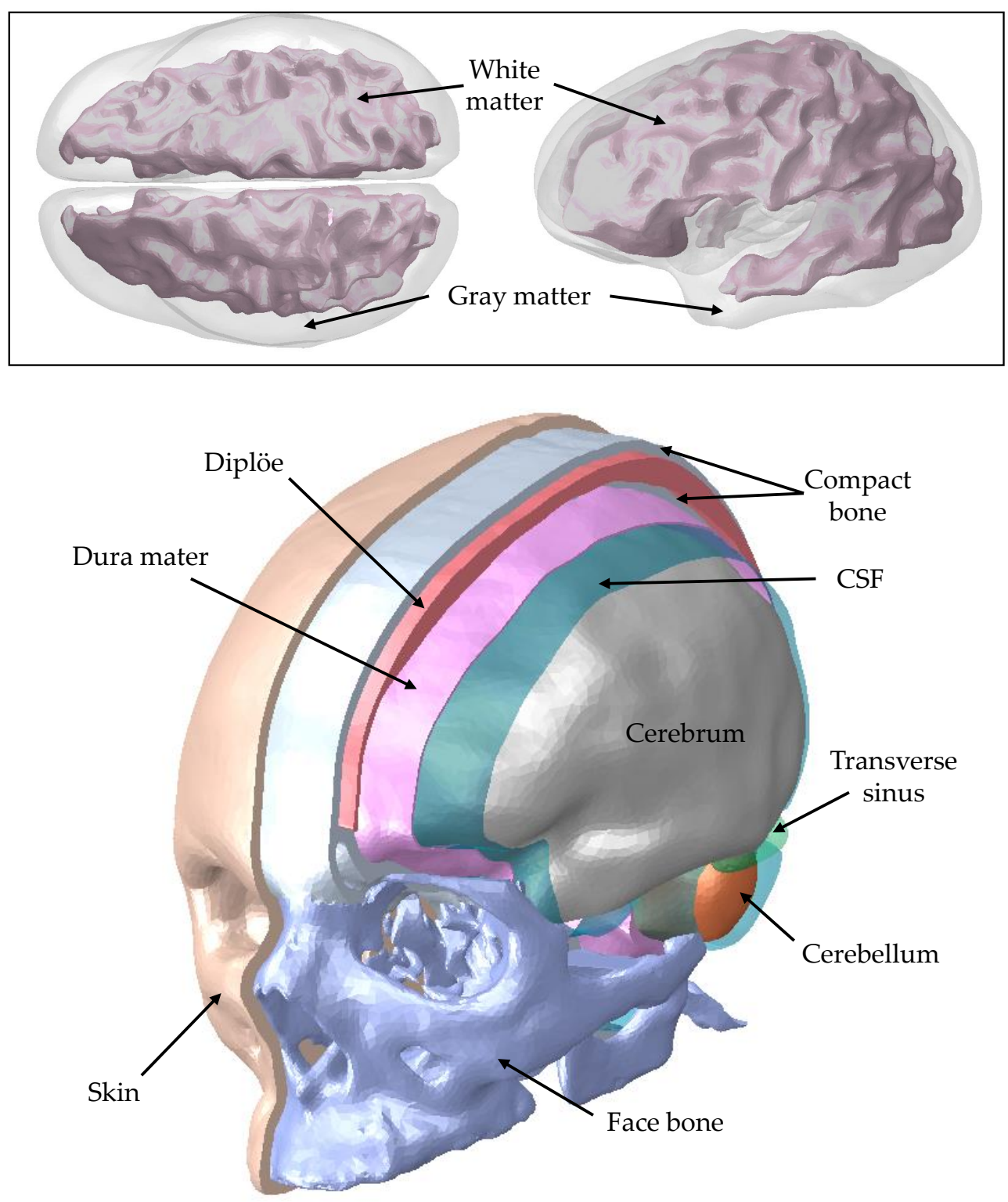

Figure 8.2: Other constituents of the current FE head model. Top: cerebrum divided into white and gray matter. Bottom: bony structures, dura matter and CSF layer. 
Chapter 8. Numerical modeling of a human head with further anatomical 202 details

The less visible parts in the DICOM images were the fluid cavities: ventricles and sinuses. These have been manually modeled and, to ensure an adequate size for each of them, the volume inside each cavity has been measured and compared to experimental data obtained from the literature. Matsumae et al. (1996) [255] measured this volume values from MR images of 49 human subjects and reported a mean ventricular volume of $25 \pm 11 \mathrm{~cm}^{3}$ and $157 \pm 59 \mathrm{~cm}^{3}$ for cranial CSF. In the model developed in this chapter, the sum of volumes of the left, right and third ventricles is $25.63 \mathrm{~cm}^{3}$, inside the range given by [255]. In the case of CSF, the calculated volume amounts to 252.2 $\mathrm{cm}^{3}$, higher than the average in [255]. Nonetheless, this difference may be due to the fact that in the study by Matsumae et al. the CSF volume trapped in the sulci is not included in the cranial CSF volume, whereas in our head model there is no distinction between both. The vasculature has been omitted following the trend of most of the head numerical models employed at present.

\begin{tabular}{lccc}
\hline & $\rho\left(\mathrm{kgm}^{-3}\right)$ & $E(\mathbf{M P a})$ & $\nu$ \\
\hline Compact bone & 1800 & 15000 & 0.21 \\
\hline Diplöe & 1500 & 4500 & 0.22 \\
\hline Facial bones & 3000 & 5000 & 0.21 \\
\hline Scalp & 1130 & 16.7 & 0.42 \\
\hline Falx/Tentorium & 1130 & 31.5 & 0.45 \\
\hline Dura matter & 1130 & 31.5 & 0.45 \\
\hline
\end{tabular}

Table 8.1: Mechanical properties of the head tissues assumed as linear elastic in the numerical model.

The material properties set for some of the head tissues are summarised in Table 8.1. The bony structures are modeled as linear elastic materials, and a user subroutine for element deletion is implemented for the skull layers based on the maximum stress criteria, with ultimate stresses of 90/-132 and 34.8/$24.8 \mathrm{MPa}$ for compact bone and diplöe, respectively. This constitutive laws have been inherited from the previous head model presented in this Thesis. 
Gray matter White matter Brainstem

\begin{tabular}{lccc}
\hline Density $\left(\mathrm{kgm}^{-3}\right)$ & 1004 & 1000 & 1000 \\
\hline \multirow{4}{*}{ Hyperelasticity } & Mooney-Rivlin & Ogden & Mooney-Rivlin \\
& $\mathrm{C}_{10}=7.8 \mathrm{kPa}$ & $\mu=3.56 \mathrm{kPa}$ & $\mathrm{C}_{10}=14 \mathrm{kPa}$ \\
& $\mathrm{C}_{01}=-1.03 \mathrm{kPa}$ & $\alpha=6.101$ & $\mathrm{C}_{01}=-1.9 \mathrm{kPa}$ \\
\hline \multirow{4}{*}{ Viscoelasticity } & Prony series & Prony series & Prony series \\
& $\mathrm{g}_{1}=0.8$ & $\mathrm{~g}_{1}=0.8$ & $\mathrm{~g}_{1}=0.8$ \\
& $\mathrm{~g}_{2}=0.16$ & $\mathrm{~g}_{2}=0.16$ & $\mathrm{~g}_{2}=0.16$ \\
& $\tau_{1}=5 \mathrm{~s}$ & $\tau_{1}=5 \mathrm{~s}$ & $\tau_{1}=5 \mathrm{~s}$ \\
& $\tau_{2}=200 \mathrm{~s}$ & $\tau_{2}=200 \mathrm{~s}$ & $\tau_{2}=200 \mathrm{~s}$ \\
\hline
\end{tabular}

Table 8.2: Mechanical properties of the different constituents of the brain applied to the head model.

The mechanical properties chosen for the constituents of the brain can be found in Table 8.2. Other intracranial structures derived from the spinal chord share the mechanical properties of either the gray or the white matter. For instance, the corpus callosum adopts the properties of the white matter while the thalamus is a gray matter structure. The brainstem, which accounts for a highly oriented axonal lattice in the longitudinal direction, has been considered stiffer than the other white-matter structures.

The sinuses, ventricles and intracranial space have been modeled as filled with fluid by using the fluid cavity option in Abaqus/Explicit. This is a novel approach which avoids meshing the interior of the cavities and the assumption of blood and the CSF as elastic fluid-like materials. The fluid flow and exchange between these regions has not been modeled in order to save computational costs and because its influence may not be substantial for the subsequent calculations. However, a certain initial pressure has been assumed inside these cavities to mimic the real conditions of a living head.

\subsection{Validation}

With the aim of ensuring an accurate response of the developed model in terms of intracranial displacements and brain strains, which are relevant for further 
Chapter 8. Numerical modeling of a human head with further anatomical 204 details

evaluations of axonal injuries, two experimental works from the literature have been taken and reconstructed numerically in Abaqus/Explicit.

The first experimental test for validation has been taken from the work by Hardy et al. (2001) [256] which consisted of applying impacts of variable magnitudes at different locations of postmortem human heads.

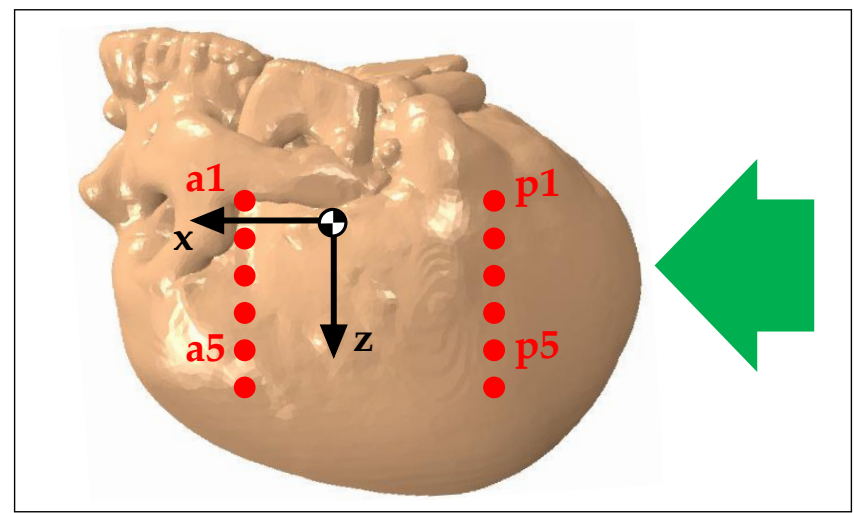

Figure 8.3: Scheme of the experimental test C755-T2 from [256], corresponding to an occipital blow. The NDTs where relative displacement is measured are depicted in red colour.

In the tests from [256], the specimen head is placed upside down and struck by an impactor either at the front or the occiput. The relative motion between the brain and the skull is obtained by recording the position of several Neutral Density Targets (NDTs) placed in two columns at the parietal side of the cerebrum. The location of these targets and the local reference system employed to compute the motion histories are depicted in Fig.8.3. All the relative displacements are recorded according to the inertial reference system illustrated in Fig.8.3, whose origin is the center of gravity of the head and the $\mathrm{Y}$ axis is parallel to the left-right direction, in such a way that the X-Z plane coincides with the sagittal plane. The $\mathrm{X}$ axis points to the front while $\mathrm{Z}$ points to the vertex of the head. Specifically, the test that will be simulated in this section is labelled in [256] as C755-T2 and corresponds to an occipital blow in the antero-posterior direction.

The second experimental work taken for numerical reconstruction is the one conducted by Hardy et al. (2007) [257], which follows the same proce- 
dure as [256] but includes some additional data such as lateral impacts and a measure of the strains substained by the brain tissue. For its recreation in Abaqus/Explicit, the experimental test labelled in [257] as 380-T1 has been taken. It consists of a lateral impact delivered in the upper parietal left region, as depicted in Fig. 8.4. The experimental data from this test will be employed to validate the behaviour of the head model presented in this chapter under lateral loading. It represents an interesting feature in order to validate the representation of the falx cerebri, a membrane which can act as a barrier to the free movement of the brain tissue.

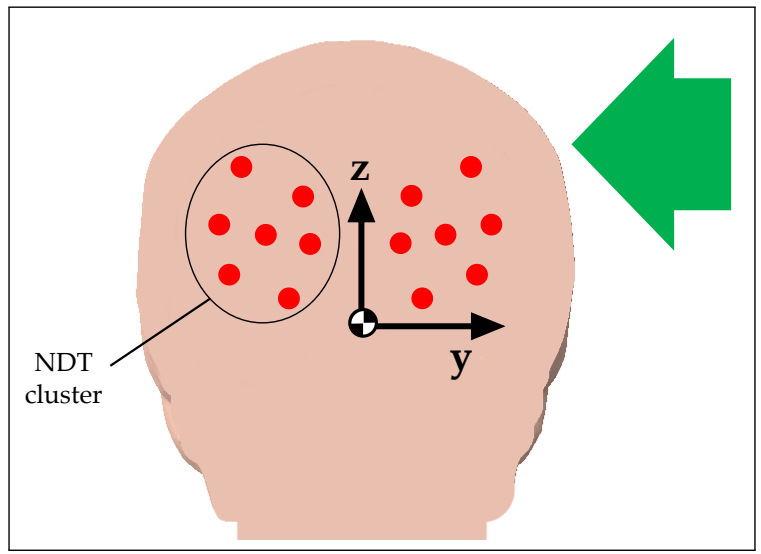

Figure 8.4: Cut in the head's coronal plane with the scheme of the experimental test C383-T1 from [257], corresponding to left parietal blow. The NDTs are clustered in groups of 7 (red dots).

To ensure the match between the kinematics of the experiment and the model, the resultant translational and rotational accelerations obtained in [256] and [257], respectively, (plotted in Fig. 8.5) are set as boundary conditions to the center of gravity of the head model in the local reference system. This method also involves a reduced computational cost as it is not necessary to model the impact environment, which deals with additional boundary conditions, and impacting parts that would require contact interactions. For instance, test $380-\mathrm{T} 1$ is carried out upon a head protected with a helmet, but the interest in this section is focused on the displacements and strains of the brain tissue rather than the effect of impact attenuation of the helmet itself. Therefore, by setting the resultant acceleration histories at the center of grav- 
Chapter 8. Numerical modeling of a human head with further anatomical 206 details

ity of the head as boundary conditions, the problem would be simplified and properly defined.
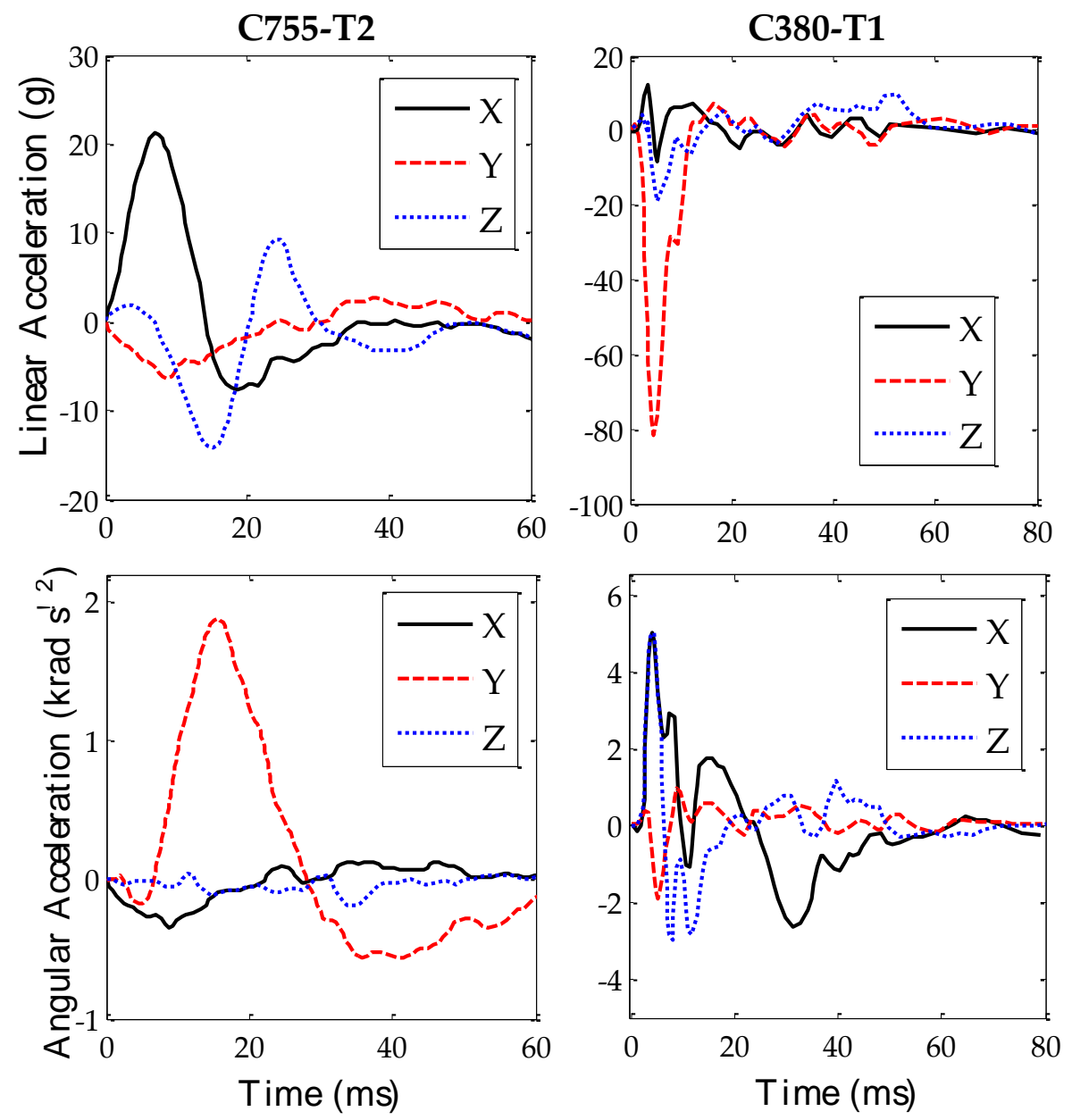

Figure 8.5: Boundary conditions applied to the six degrees of freedom of the head model for experiments C755-T2(top) and C383-T1(bottom) from [256]. 


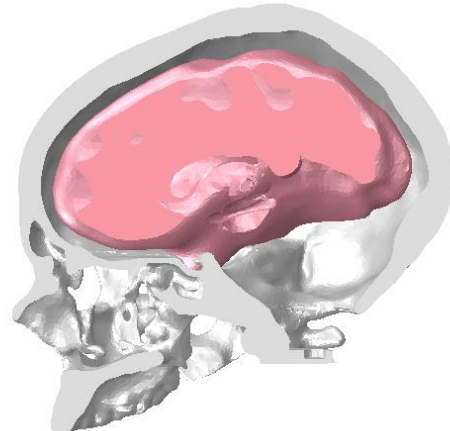

$$
\mathrm{t}=0 \mathrm{~ms}
$$

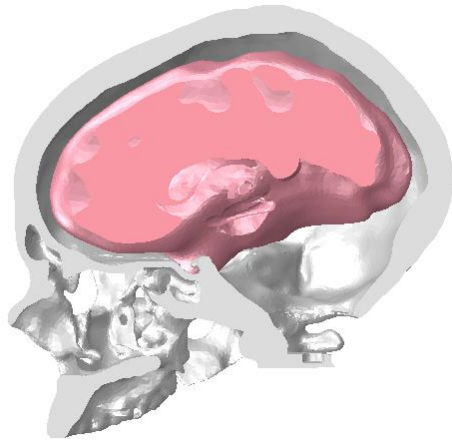

$$
\mathrm{t}=12 \mathrm{~ms}
$$

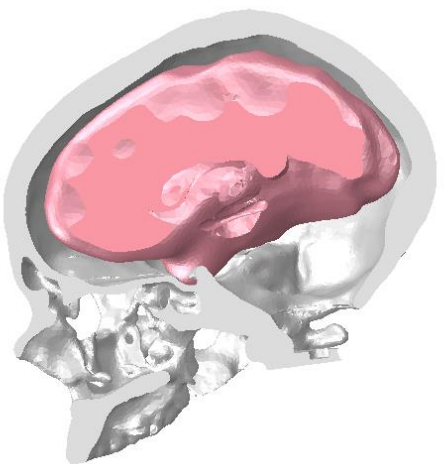

$$
\mathrm{t}=24 \mathrm{~ms}
$$
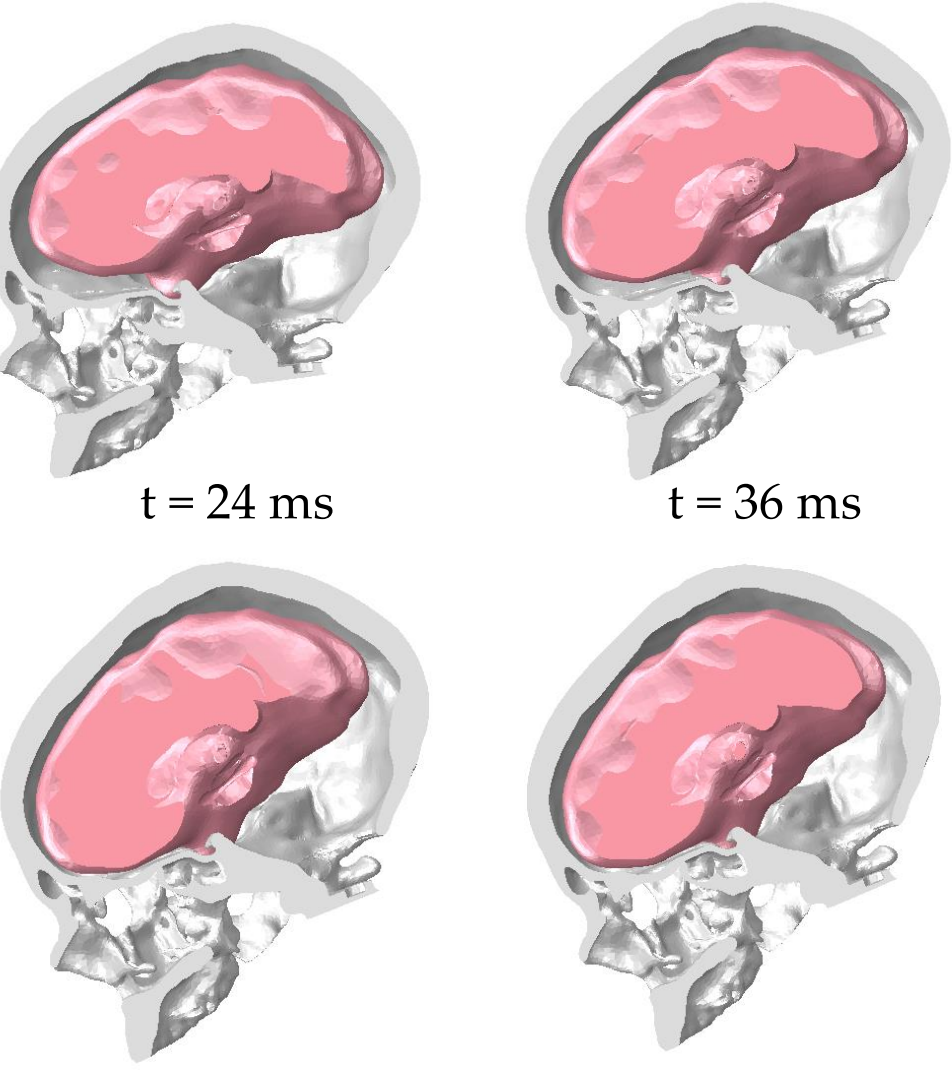

$$
\mathrm{t}=48 \mathrm{~ms}
$$

$$
\mathrm{t}=60 \mathrm{~ms}
$$

Figure 8.6: Relative brain-skull displacement obtained from the FE simulation of test C755-T2 from [256]. 
Chapter 8. Numerical modeling of a human head with further anatomical 208 details

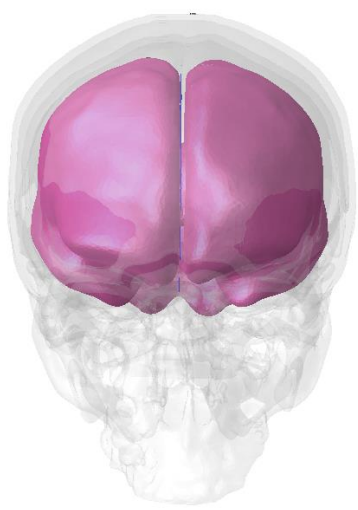

$$
\mathrm{t}=0 \mathrm{~ms}
$$

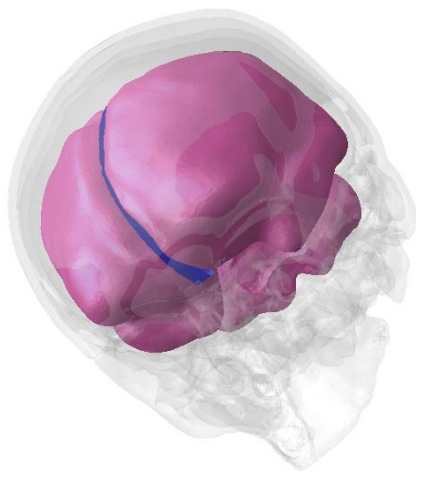

$\mathrm{t}=45 \mathrm{~ms}$

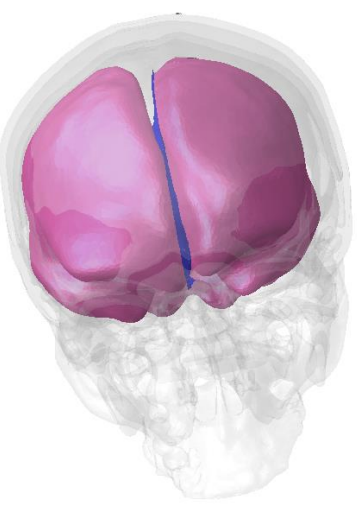

$\mathrm{t}=15 \mathrm{~ms}$

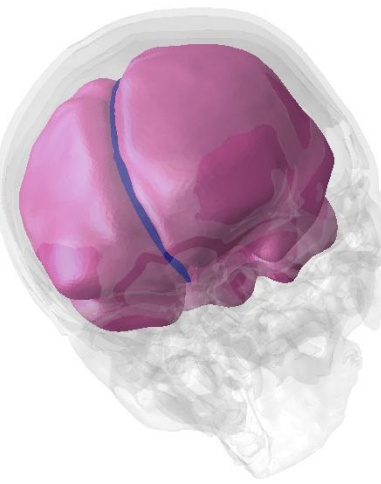

$\mathrm{t}=60 \mathrm{~ms}$

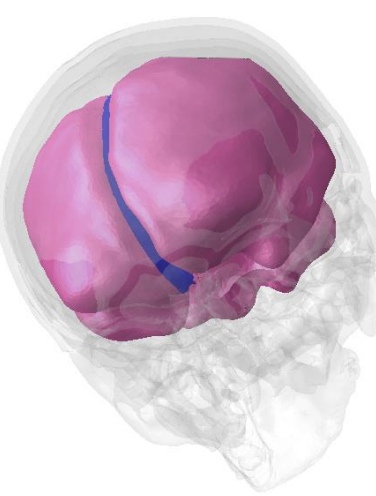

$\mathrm{t}=30 \mathrm{~ms}$

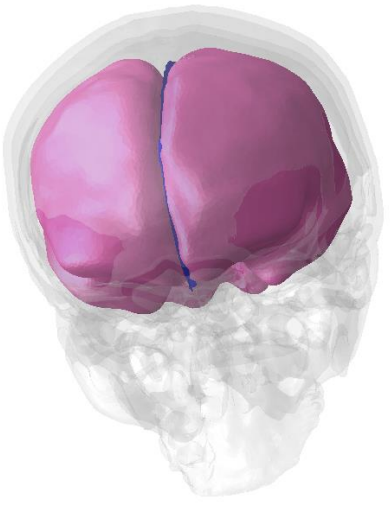

$\mathrm{t}=75 \mathrm{~ms}$

Figure 8.7: Brain motion subsequent to the lateral impact test C383-T1 from [256].

Relative skull-brain displacements calculated from the simulation of test C755-T2 in the $\mathrm{X}$ and $\mathrm{Z}$ directions are plotted in Fig.8.8. Good agreement is found between the experimental and predicted response, with an average maximum displacement overprediction of the $15 \%$, which is assumable given the differences in geometrical features and the complex behaviour of the cerebral tissues. 

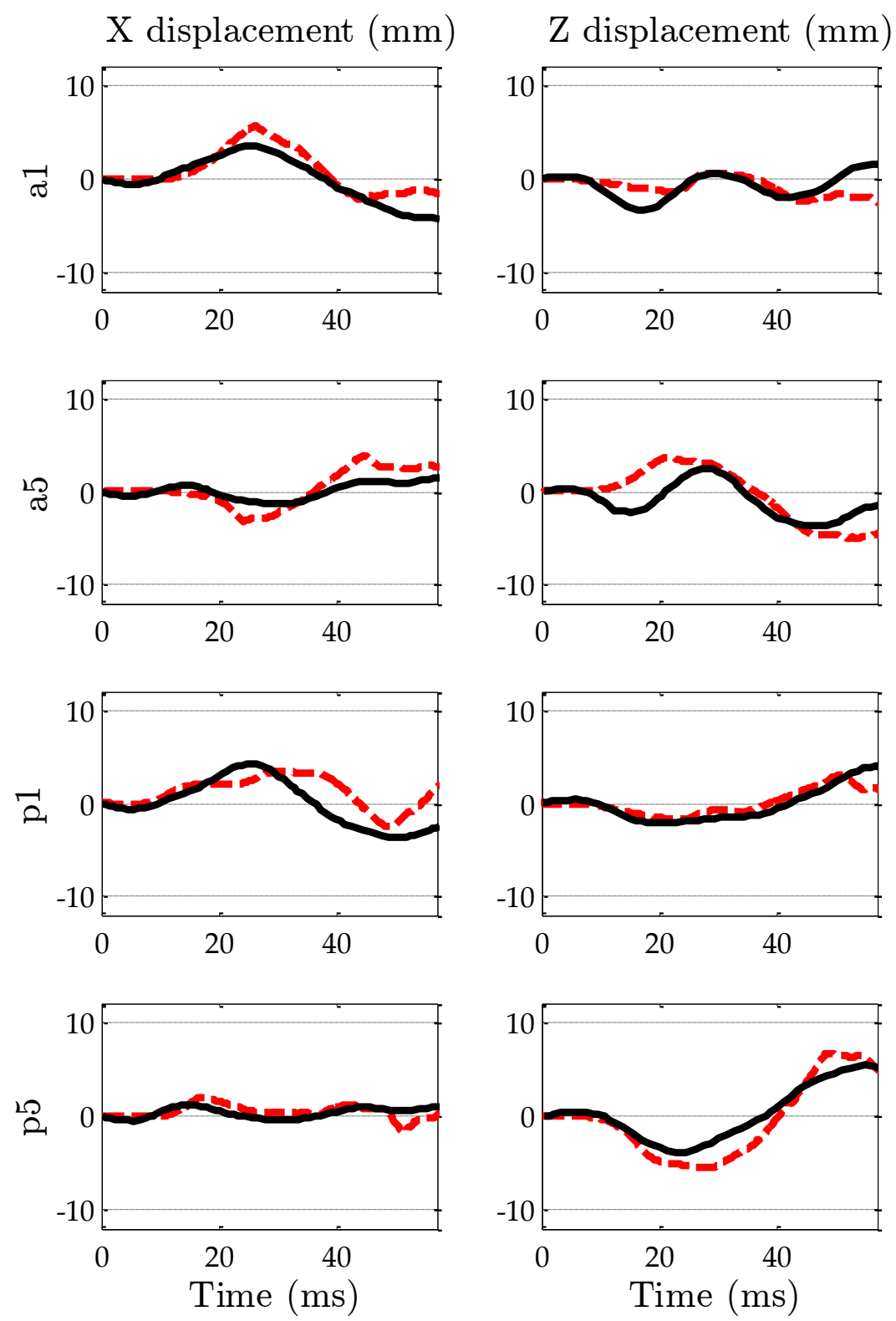

-ーー-Simulated $\longrightarrow$ Experimental

Figure 8.8: Simulated and experimental relative displacements in the $\mathrm{X}$ and $\mathrm{Z}$ directions for the NDTs reported in test C755-T2 from [256]. 
Chapter 8. Numerical modeling of a human head with further anatomical 210 details

The same agreement between the experimental and simulated response has been achieved for test C380-T1 (Fig. 8.9) suggesting a proper modeling of the inner soft tissues involved in the lateral movement response of the head.
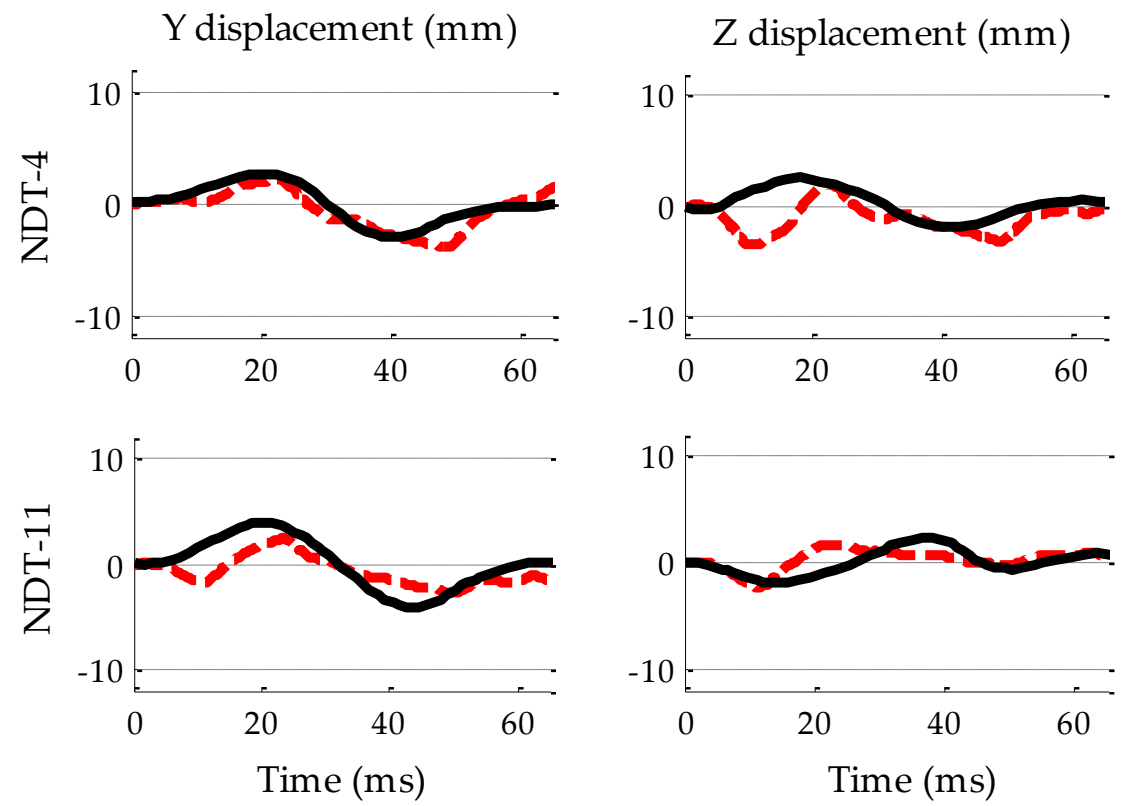

- - Simulated

Experimental

Figure 8.9: Simulated and experimental relative displacements in the $\mathrm{Y}$ and $\mathrm{Z}$ directions for the NDTs reported in test C380-T1 from [256]. 


\subsection{Application to an accident reconstruction}

The head injury risk associated to ballistic impact using personal protections was assessed in this Thesis by means of the head model from Chapter 5. However, the head model presented in this chapter offers additional possibilities of employment. One area in which numerical modeling has emerged as an invaluable tool is the reconstruction of accidental environments. A full understanding of the impact scenario through FE reconstruction can complement the medical diagnostic and the prognosis of the patient.

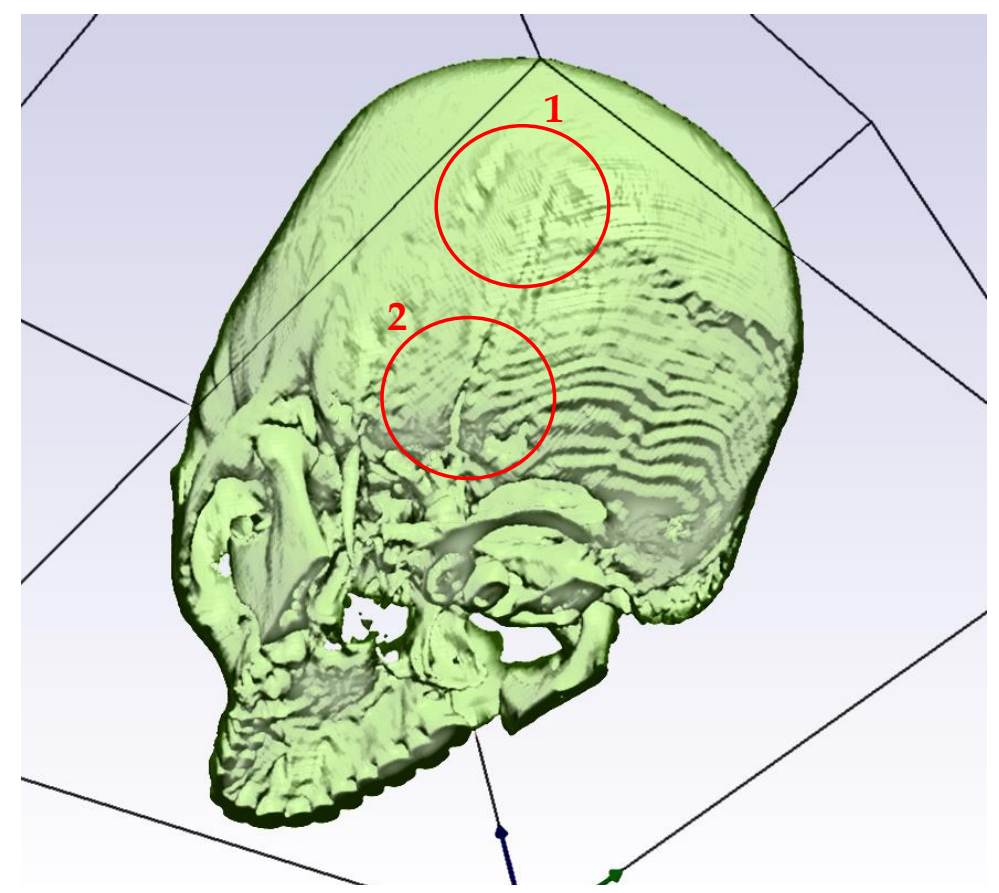

Figure 8.10: Reconstruction of the cranial fractures from the CT images with ScanIP.

A real-case equestrian accident has been simulated in Abaqus/Explicit. By previously knowing the lesions suffered by the patient, it will be possible to infer some mechanical-based criteria for such injury mechanisms. Very little information regarding the environment of the accident was available. The patient presented an acute subdural hematoma (SDH) and a depressed skull 
Chapter 8. Numerical modeling of a human head with further anatomical

fracture in the left temporo-occipital region (Fig. 8.10 (1)) accompanied by a linear fracture which extends towards the base of the skull (Fig. 8.10 (2)). Also, contusive focuses were found in the frontal lobe including subarachnoid haemorrhage $(\mathrm{SAH})$.

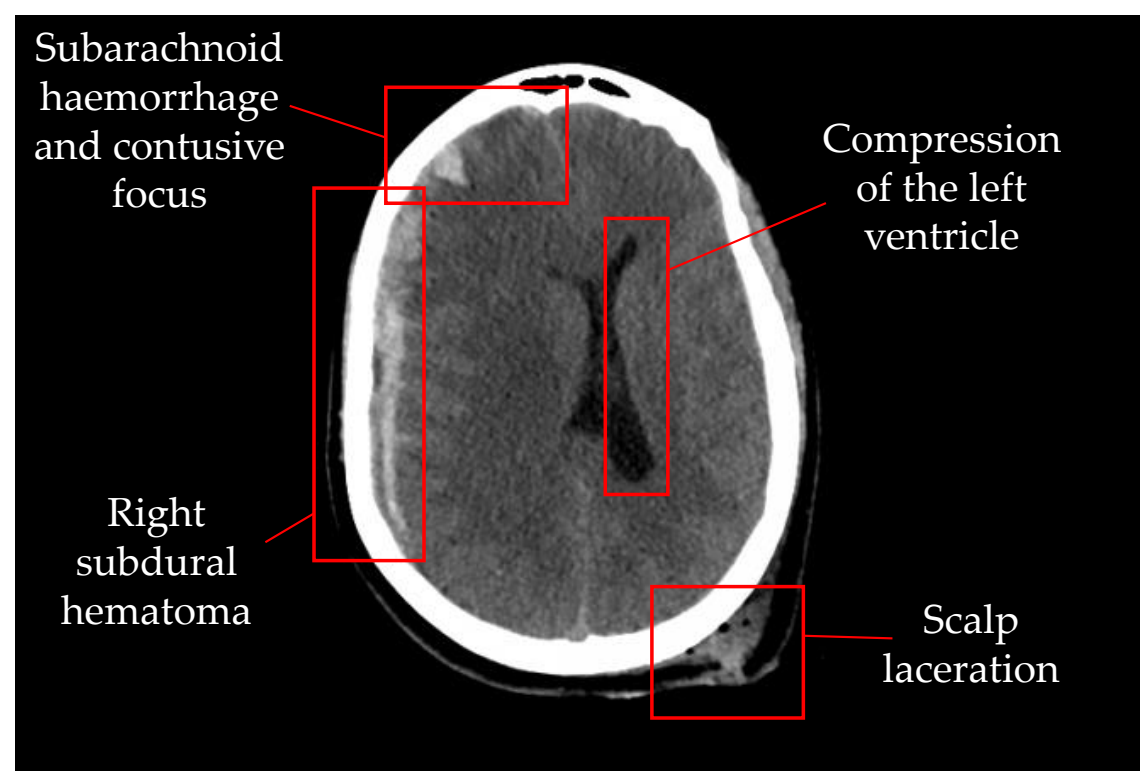

Figure 8.11: Imaging of the intracranial lesions after impact.

A simulation in Abaqus/Explicit is performed recreating the accident by means of a rigid flat surface striking the head model at a given initial velocity. This velocity is estimated based on the approximate height at the beginning of the fall. A boundary condition is set to the first cervical vertebra in order to impose the restrictions that would involve the presence of the neck and the rest of the body. Further adjustments to the initial position and velocity of the plate were performed in order to match the clinically observed scalp laceration and skull fracture pattern. Fig. 8.12 shows the equivalence between the clinical cranial damage and the FE reconstruction. 

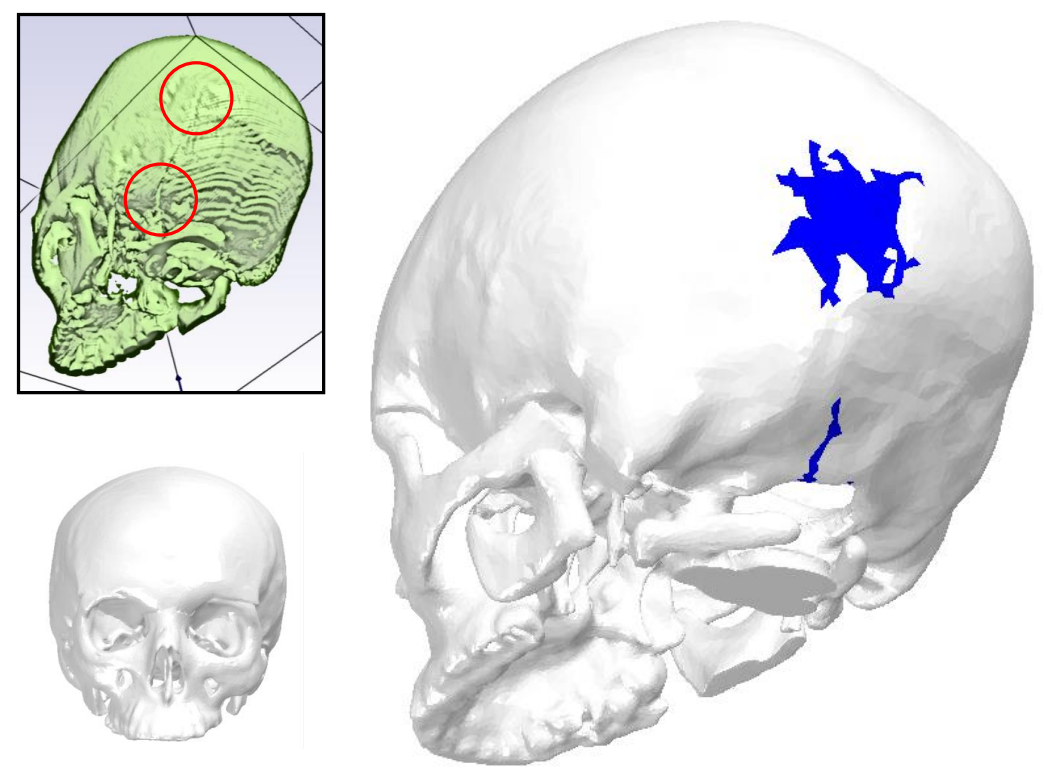

Figure 8.12: Cranial fracture obtained through numerical simulation matching the patterns observed in the original CT scan of the patient.

\subsection{Prediction of subdural hematoma}

As explained in the section regarding head injuries at the second chapter of this Thesis, subdural hematoma (SDH) is considered to be caused by the rupture of the bridging veins (BV) that connect the superior sagittal sinus (SSS) with the surface of the brain. This rupture provokes the drainage of blood inside the intracranial cavity.

In the clinical case studied in this section, a right subdural hematoma is detected. Lee and Haut (1989) [258] suggested that occipital impacts were more prone to cause SDH as the bridging veins, due to their orientation, would undergo tension at the first stage of the impact. From experimental impact tests on rhesus monkeys, Ommaya et al. (1971) [149] found a higher ocurrence of SDH in the anterior half of the brain and stated a high probability of SDH under an impact scenario involving occipital skull fracture. Taking into account the skull fracture pattern from the patient and the scalp laceration, it can be hipotesised that the impact took place at the left-occipital 
Chapter 8. Numerical modeling of a human head with further anatomical 214 details

site of the head. SDH and the contusive regions can be detected by means of CT images but this technique does not permit a detailed knowledge of the injury initiation, as the vascular tissue is hardly distinguishable from the surrounding meninges and brain $[259,260]$. Departing from this initial drawback, a three-dimensional reconstruction of the intracranial contents based on the available CT images from the patient has been performed using the open software 3DSlicer. Fig. 8.13 shows the upper cranial portion including the dura mater, superior sagittal sinus and the most cortical part of the hematoma.

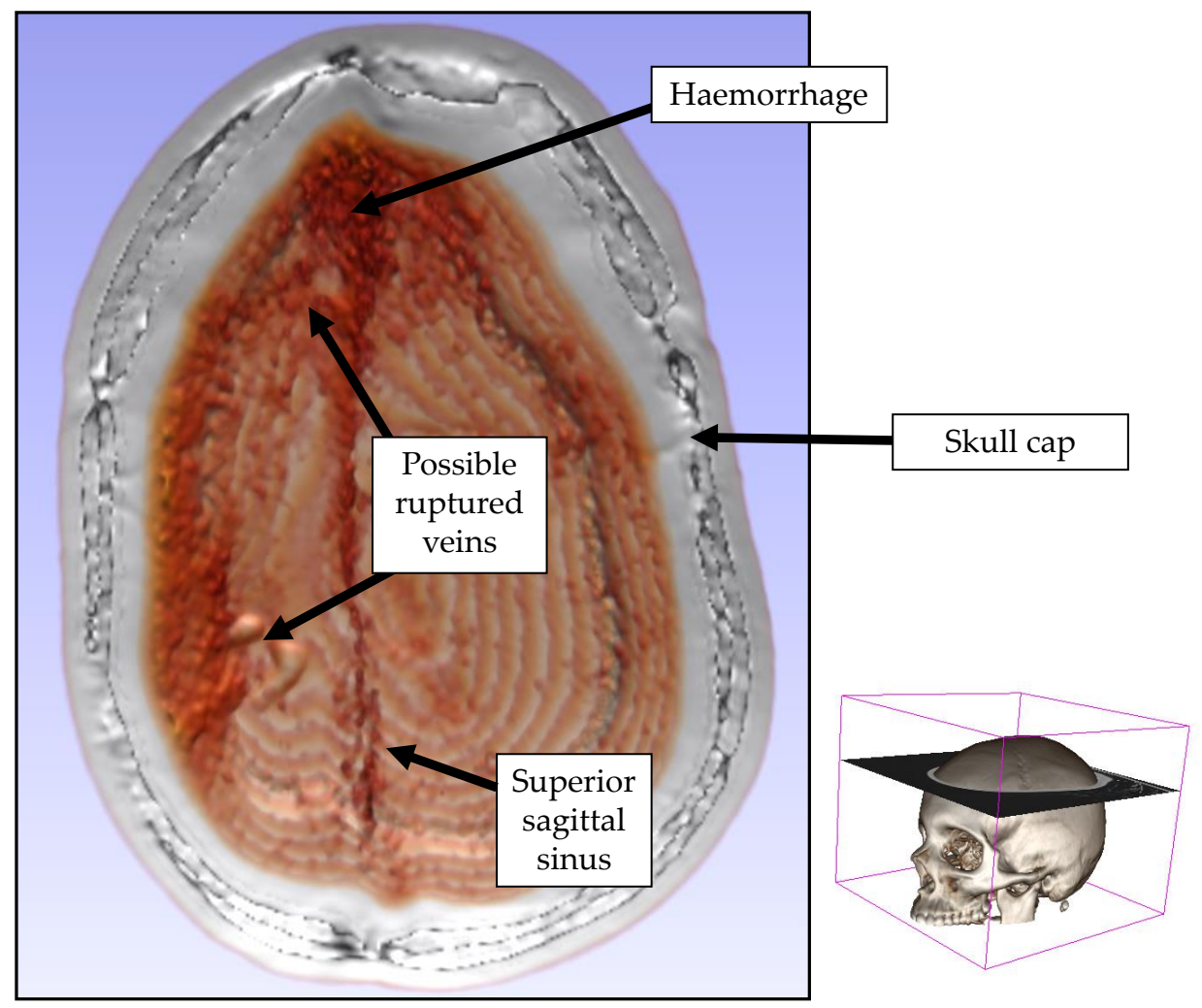

Figure 8.13: Rendered view of the top skullcap from the CT scan. Intense red areas indicate blood accumulation representing the $\mathrm{SDH}$ and $\mathrm{SAH}$.

From this reconstruction two possible sites of blood drainage are visible: the first one, at the frontal site, in one of the antegrade veins, and the second 
one in the parietal site, as the volume reconstruction revealed two protuberances in the dura mater which may indicate the presence of thrombos. Ehrlich et al. (2001) determined from their X-ray examinations of lethal head injuries that frontal bridging veins are the most frequently ruptured in impact, probably due to their entry angle to the SSS. Nonetheless, Knowles et al. (2017) [261] performed a FE study employing the SIMon model and obtained greater strain values in the posterior veins for occipital impacts. Also, it has been extensibly hypotesised in the literature that maximum relative skull-brain displacements tend to be reached at the vertex region due to the constraint to motion at the brain's base (along the posterior fossa) [262, 263].

Some existing head FE models in the literature explicitly model the parasagittal bridging veins complex employing one-dimensional elements [128, $211,261]$. In the present study, the baseline model presented at the beginning of this section does not account for the presence of these vessels. Given that the stiffness of these structures is not negligible and may restrict the relative brain-skull movement, a second model including a simplified representation of these veins has been constructed.

Three simulations of the fall are carried out: first with the baseline model, the second with the model including the bridging veins and a third one with the later model but without including skull fracture. The motivation for this third case is that skull fracture has been considered not to affect the triggering of SDH, which is often regarded as a merely rotation-based injury.

\subsubsection{Inclusion of the bridging veins}

Following the approaches taken in the literature, eleven pairs of beam elements (B31 in Abaqus/Explicit) have been created joining nodes from the dura mater covering the SSS and the brain surface. The orientations, lengths and diameters set to these elements have been extracted from anatomical studies [258, 264]. The mechanical properties set to the beam elements have been taken from the experimental work of Monea et al. (2014) [140], which establishes an average Young's modulus of the vessel of $25.72 \mathrm{MPa}$. A schematic view of the resulting vascularity in the head model is given in Fig.8.14. 
Chapter 8. Numerical modeling of a human head with further anatomical

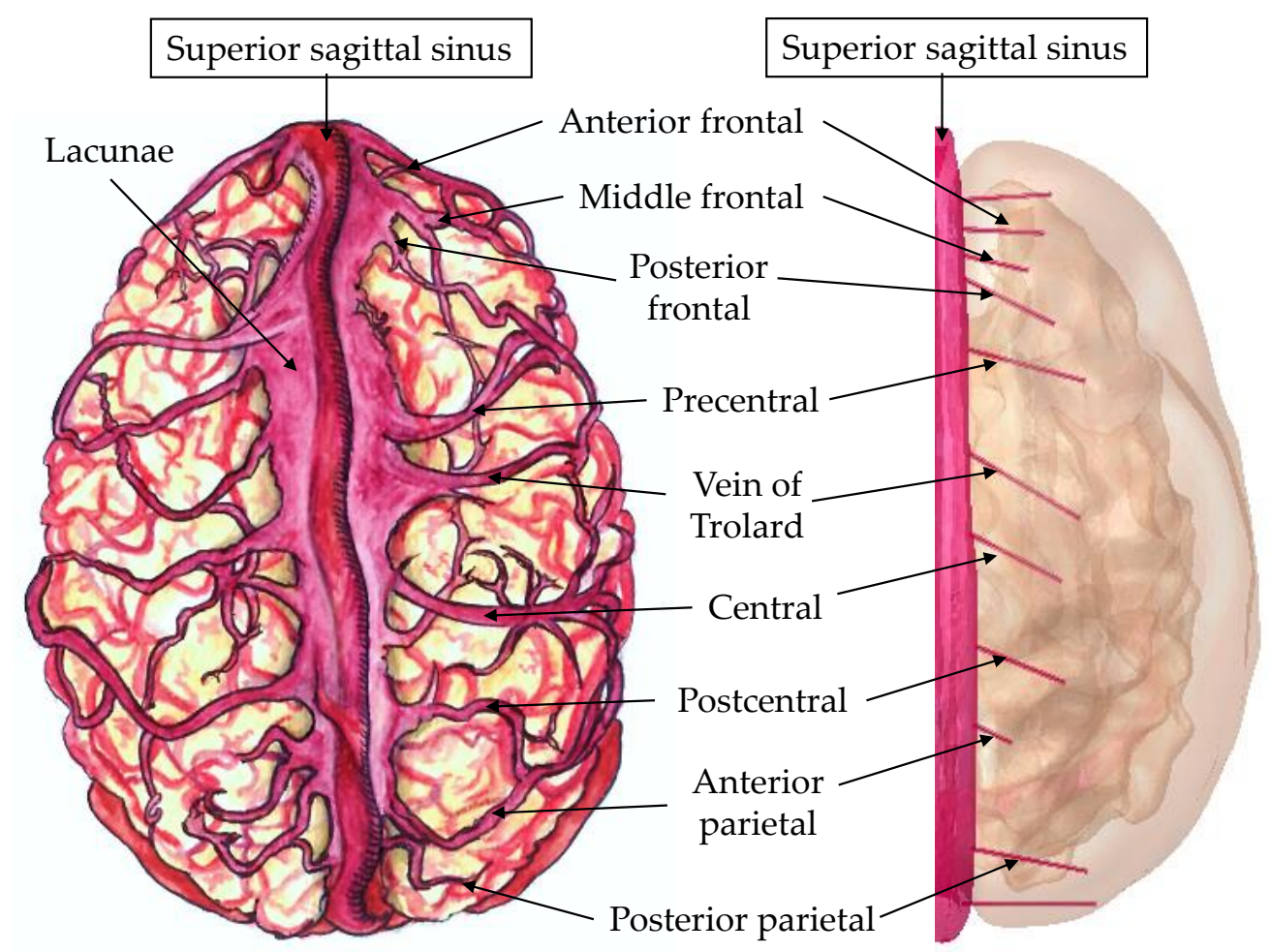

Figure 8.14: Anatomical distribution of the bridging veins in a real head (left) and their simplified architecture for the numerical model of this section (right).

One major point of getting a comparison between both models (with and without BV) is to quantify the influence of including this vasculature into the overall skull-brain movement. After performing both simulations, the displacement fields at the brain at the moment of maximum brain-skull relative displacement have been obtained and represented in Fig. 8.15.

It can be observed that both displacement fields along the brain and SSS present very similar distributions and close maximum values, being the maximum difference of $1.5 \mathrm{~mm}$. The most remarkable variances can be found, as expected, in the brain regions close to the SSS, as the bridging veins are providing additional stiffness in this area of the subarachnoid space. Although the relative displacement brain-skull remains almost identical in the anteroposterior direction in both cases, lateral movement of the brain hemispheres is 
constrained by the traction forces supported by the blood vessels in the model with BV. As a result, in the case studied in this section of left occipital impact, during the acceleration stage of the loading the left hemisphere loads the frontal and central bridging veins axially in tension, and these retain the inertial movement of the cerebrum. Conversely, at the deceleration stage of the impact, the movement of the right hemisphere towards the skull is constrained by the central and posterior vessels.
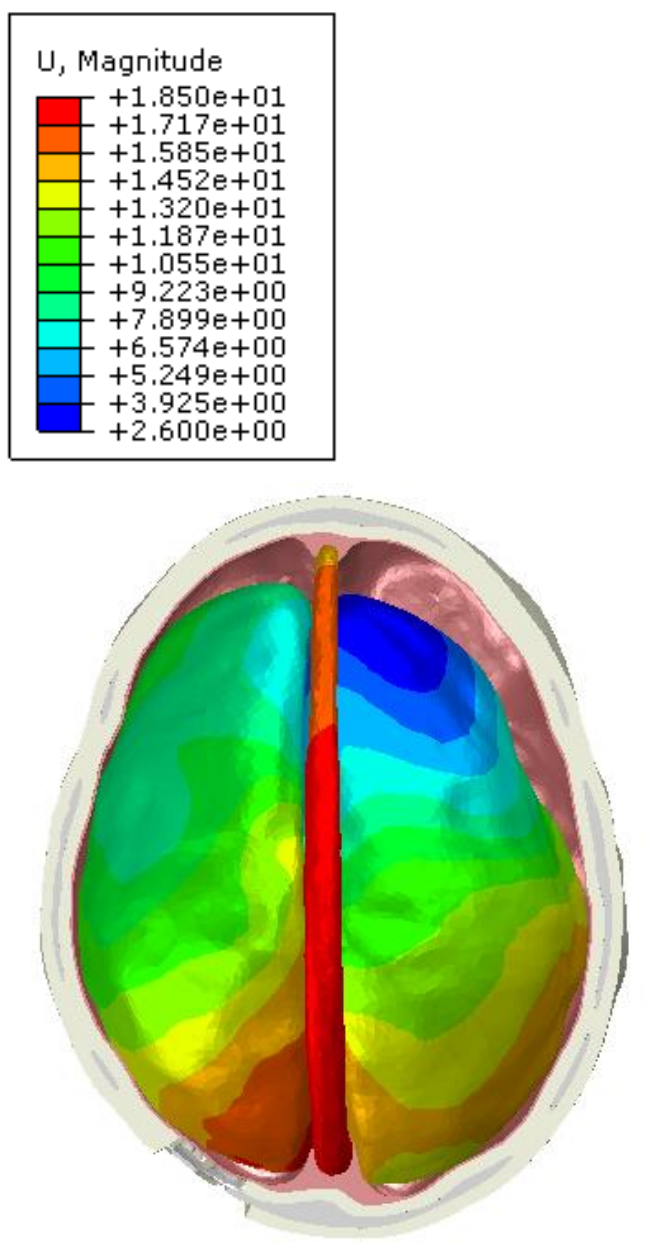
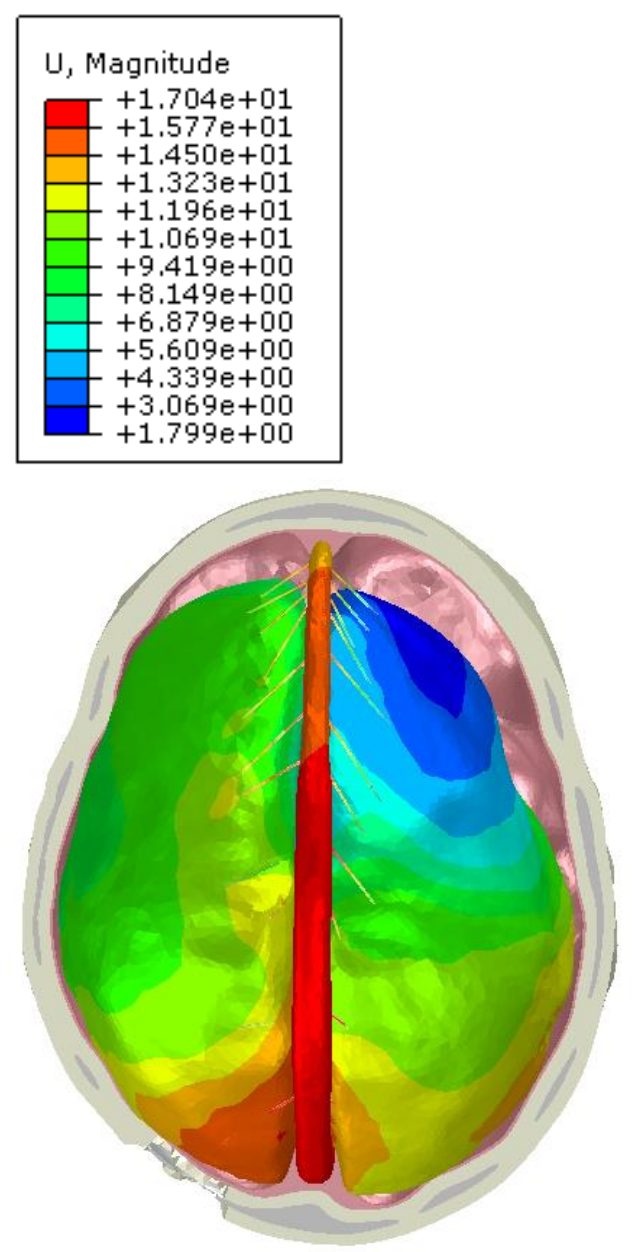

Figure 8.15: Maximum relative displacement between the brain and the SSS from the baseline model (left) and the model including the BV (right). 
Chapter 8. Numerical modeling of a human head with further anatomical

Left hemisphere

Right hemisphere

\begin{tabular}{lccc|ccc}
\hline & Baseline & With BV & Difference & Baseline & With BV & Difference \\
\hline FP & $13.60 \%$ & $2.55 \%$ & $11.05 \%$ & $-8.80 \%$ & $-3.09 \%$ & $-5.71 \%$ \\
\hline AF & $12.59 \%$ & $-3.95 \%$ & $16.54 \%$ & $-12.60 \%$ & $-7.95 \%$ & $-4.65 \%$ \\
\hline MF & $81.41 \%$ & $32.54 \%$ & $48.87 \%$ & $44.89 \%$ & $28.45 \%$ & $16.44 \%$ \\
\hline PF & $60.12 \%$ & $25.14 \%$ & $34.98 \%$ & $35.48 \%$ & $21.70 \%$ & $13.78 \%$ \\
\hline PC & $54.63 \%$ & $27.43 \%$ & $27.20 \%$ & $-1.31 \%$ & $-3.17 \%$ & $1.86 \%$ \\
\hline VT & $46.72 \%$ & $20.37 \%$ & $26.35 \%$ & $11.86 \%$ & $7.09 \%$ & $4.77 \%$ \\
\hline C & $53.13 \%$ & $37.58 \%$ & $15.55 \%$ & $-0.91 \%$ & $-6.93 \%$ & $6.02 \%$ \\
\hline PsC & $47.22 \%$ & $39.41 \%$ & $7.81 \%$ & $8.48 \%$ & $3.53 \%$ & $4.95 \%$ \\
\hline AP & $75.42 \%$ & $67.20 \%$ & $8.22 \%$ & $44.68 \%$ & $31.20 \%$ & $13.48 \%$ \\
\hline PP & $24.13 \%$ & $23.47 \%$ & $0.66 \%$ & $12.96 \%$ & $11.08 \%$ & $1.88 \%$ \\
\hline O & $7.74 \%$ & $3.29 \%$ & $4.45 \%$ & $8.10 \%$ & $7.63 \%$ & $0.47 \%$ \\
\hline
\end{tabular}

Table 8.3: Predicted principal strains on the bridging veins complex during impact loading $(\mathrm{t}=4.5 \mathrm{~ms})$ in the baseline model and the model with $\mathrm{BV}$. $\mathrm{FP}=$ frontopolar, $\mathrm{AF}=$ anterior frontal, $\mathrm{MF}=$ middle frontal, $\mathrm{PF}=$ posterior frontal, $\mathrm{PC}=$ precentral, $\mathrm{VT}=$ vein of Trolard, $\mathrm{C}=$ central, $\mathrm{PsC}=$ postcentral, $\mathrm{AP}=$ anterior parietal, $\mathrm{PP}=$ posterior parietal, $\mathrm{O}=$ occipital.

This reduction in relative displacement has been quantified for discrete pairs of nodes at the surface of the cerebral hemispheres close to the sagittal plane and the dura mater surrounding the superior sagittal sinus, corresponding to the edges of the bridging veins. The resultant virtual strain values for each vessel, calculated as the initial distance between nodes by the distance at time $t=4.5 \mathrm{~ms}$, are reported in Table 8.3.

Despite the geometrical simplicity of the BV model, its introduction to the overall head model does have a constraint effect in the relative movement of the brain hemispheres with respect to the SSS and it seems reasonable to maintain this configuration for the subsequent evaluation of SDH. A recent study by Migueis et al. [265] detailedly modeled the three-dimensional characteristics 
of the BV using solid elements with an elastoplastic behaviour coupled to a ductile damage law. Despite being able to recreate the actual rupture of the vessels, they were mostly located at the mid-body of the vessel, which is not a reallistic approach of SDH initiation, as it develops from ruptures at the SSS entry. This fact suggests that explicit modeling of the BV may not be so accurate in injury prediction to compensate the added computational expense.

In order to achieve a qualitative validation of the behaviour of the beamlike BV in conjunction to the baseline head model for the prediction of SDH, the experimental work by Depreitere et al. (2006) [137] will be employed as a reference. This is, up to date, the only study explicitly addressing BV ruptures on PMHS. Apart from providing the kinematic conditions measured at their experimental tests (angular and translational accelerations and pulse duration), the exact location of the ruptured vessels is achieved by means of fluoroscopy. Some of the tests reported in [137] have been recreated with the head model presented in this section. The kinematic boundary conditions set to each simulations are gathered in Table 8.4.

\begin{tabular}{lllll}
\hline $\begin{array}{l}\text { Test } \\
\text { ID }\end{array}$ & $\begin{array}{l}\text { Peak linear acceler- } \\
\text { ation }\left(\mathrm{ms}^{-2}\right)\end{array}$ & $\begin{array}{l}\text { Peak rotational } \\
\text { acceleration } \\
\left(\mathrm{rads}^{-2}\right)\end{array}$ & $\begin{array}{l}\text { Pulse } \\
\text { duration } \\
(\mathrm{ms})\end{array}$ & $\begin{array}{l}\text { Vein } \\
\text { rupture }\end{array}$ \\
\hline D1 & 4522 & 13411 & 5.2 & left \\
D2 & 2161 & 5777 & 4.5 & none \\
D3 & 1522 & 9445 & 15.4 & right \\
D4 & 3090 & 10607 & 7.8 & bilat \\
D5 & 3676 & 7925 & 12.0 & none \\
D6 & 2354 & 8567 & 7.7 & right \\
\hline
\end{tabular}

Table 8.4: Kinematic boundary conditions from the tests performed by Depreitere et al. [137] simulated in this section.

Before presenting the results for these simulations, it is convenient to mention the work by Musigazi and Depreitere [266], who studied the BV anatomy of 78 patients by means of CT angiography, reporting a large dispersion in both the location and entry angles of the vessels with respect to the SSS. Based on these data, a recent work by Kapeliotis et al. [267] analysed the influence of these variations on the numerical prediction of acute SDH by implementing 
Chapter 8. Numerical modeling of a human head with further anatomical

different configurations of the BV complex to their baseline head FE model from the KTH [234]. In [267], the experimental data taken as a reference is the work by Depreitere [137] which has been employed in this section. After submitting each of their four models to the kinetic boundary conditions from the tests in [137], the authors reported great differences between them in terms of the strain output of the vessels, thus confirming that the onset of $\mathrm{BV}$ rupture is highly subject-specific.

With the numerical model presented in this chapter, the results of the reconstructed experimental tests from Depreitere [137] are the following. For $\mathrm{D} 1$, the vessel sustaining the greatest stress $(3.65 \mathrm{MPa})$ is the left precentral vein, at a time of $7 \mathrm{~ms}$. In case D3, the maximum principal stress in the BV is found at the right vein of Trolard with a value of $3.74 \mathrm{MPa}$ at a time of $4.8 \mathrm{~ms}$, coinciding with the instant of maximum angular velocity $\left(25 \mathrm{rads}^{-1}\right)$. The simulation of experiment D4 results in the greatest stresses in the vessels, reaching a value of $4.1 \mathrm{MPa}$ at the right precentral vein during the acceleration phase and of $4.5 \mathrm{MPa}$ at the left midfrontal vein during deceleration. Simulation D6 leads to a maximum stress of $3.54 \mathrm{MPa}$ at the right precentral vein. In cases D2 and D5, the experiments did not result in SDH. In the simulations performed in this section, the maximum stress values recorded at the BVs are 2.6 and $3.5 \mathrm{MPa}$, respectively. This later value is sufficiently high to cause the breakage of the vessel, even though no ruptures are reported in the experimental database [137]. Nonetheless, this specific test has been also controverted in the study by Kapeliotis et al. [267], as a false positive was predicted with the model that best correlated SDH. The authors attribute this discrepancy to a potentially significant damage sustained by the most loaded vessel that was not identified in the physical examination after impact, but became evident after a second impact of reduced magnitude that resulted in vein rupture.

Given these results from the simulation of the tests by Depreitere [137], the assumption of an ultimate stress of $3.5 \mathrm{MPa}$ for the simplified bridging veins appears realistic and falls into the range experimentally reported from uniaxial tests by Delye et al. [136] and Monea et al. [140]. Furthermore, the veins sustaining the maximum stresses in the numerical model here presented match the locations of vessel rupture reported by Depreitere [137]. This fact suggests a proper distribution and geometry of the beam elements along the SSS, despite the interspecimen variations. 


\section{Appendix: Does skull fracture affect SDH?}

The effect of skull fracture over intracranial injuries has been discussed in previous works in the literature, especially from an experimental point of view. Many severe head injuries are accompanied by depressed fractures of the cranial vault. The skull is considered as a protection for the CNS, but its attenuating effects on head trauma do not imply that this structure needs to be intact. Analogously to the previous section, the relative displacements between the SSS and several nodes at the locations of the bridging veins have been calculated for two simulated cases: the first, including the skull fractures illustrated in Fig.8.12 and the second assuming much higher ultimate stresses of the cranial bones so no skull fracture is achieved in this impact simulation. Results have been summarised in Table 8.5.

\begin{tabular}{lccc|ccc}
\hline & \multicolumn{3}{c}{ Left hemisphere } & \multicolumn{3}{c}{ Right hemisphere } \\
\hline & Fracture & w/o fract. & Difference & Fracture & w/o fract. & Difference \\
\hline FP & $2.55 \%$ & $-6.69 \%$ & $-9.24 \%$ & $-3.09 \%$ & $1.39 \%$ & $4.48 \%$ \\
\hline AF & $-3.95 \%$ & $-8.75 \%$ & $-4.8 \%$ & $-7.95 \%$ & $-4.17 \%$ & $3.78 \%$ \\
\hline MF & $32.54 \%$ & $35.58 \%$ & $3.04 \%$ & $28.45 \%$ & $39.28 \%$ & $10.83 \%$ \\
\hline PF & $25.14 \%$ & $24.86 \%$ & $-0.28 \%$ & $21.70 \%$ & $26.89 \%$ & $5.19 \%$ \\
\hline PC & $27.43 \%$ & $27.93 \%$ & $0.50 \%$ & $-3.17 \%$ & $-3.71 \%$ & $-0.54 \%$ \\
\hline VT & $20.37 \%$ & $23.05 \%$ & $2.68 \%$ & $7.09 \%$ & $9.97 \%$ & $2.88 \%$ \\
\hline C & $37.58 \%$ & $38.83 \%$ & $1.25 \%$ & $-6.93 \%$ & $-6.40 \%$ & $0.53 \%$ \\
\hline PsC & $39.41 \%$ & $44.33 \%$ & $4.92 \%$ & $3.53 \%$ & $8.48 \%$ & $4.95 \%$ \\
\hline AP & $67.20 \%$ & $80.47 \%$ & $13.27 \%$ & $31.20 \%$ & $38.40 \%$ & $7.20 \%$ \\
\hline PP & $23.47 \%$ & $23.88 \%$ & $0.41 \%$ & $11.08 \%$ & $12.61 \%$ & $1.53 \%$ \\
\hline O & $3.29 \%$ & $2.77 \%$ & $-0.52 \%$ & $7.63 \%$ & $8.28 \%$ & $0.65 \%$ \\
\hline
\end{tabular}

Table 8.5: Predicted strains on the bridging veins complex during impact loading $(\mathrm{t}=4.5 \mathrm{~ms})$ in the model with BV in the cases with and without skull fracture. 
Chapter 8. Numerical modeling of a human head with further anatomical

In the majority of the locations evaluated, the predicted relative displacement at the SSS region, which is directly related to the initiation of BV rupture, is greater for the case in which the cranial vault remains unbroken. This fact highlights the relevance of skull fracture in the dissipation of the impact energy. The energy input required for the breakage of the cranial bones is substracted from the remaining impact energy, and therefore the kinetic energy to be transmitted to the intracranial contents is lower than in the case of an unruptured skull, as the energy stored elastically in the bones will be transmitted back to the head in the impact direction.

\subsection{Towards an injury criterion}

It has been seen that there is no definite consensus about a mechanical predictor for SDH. Simulations of the experimental tests by Depreitere et al. [137] have good agreement with their reported injury outcome but do not cover a wide range of impact environments. The real-life fall accident presented in this section resembles the impact scenario from [137] but the impact location is biased to the left occipital side of the head and therefore it cannot be treated as a purely sagittal plane loading.

In the simulation of the equestrian accident considered in this chapter, the bridging veins suffering the greatest strains during the acceleration phase were the ones on the left head side, which is in contradiction with the injuries from the CT scan and the clinical report. The assumed ultimate stress value for vessel rupture is the one derived from the simulation against Depreitere [137] tests. Stresses above this threshold are also sustained by the central and postcentral veins of the right hemisphere during deceleration. The rupture of these vessels of the middle brain segment would be consistent with the clinical report. There is, however, no special reason to discard the vein ruptured at the left hemisphere, which indeed was the first to be injured during impact. This fact suggests that other injury mechanisms apart from pure traction may be involved in the BV blood leakage into the dura mater. In fact, if focusing on the anatomical features of these vessels, it can be perceived that not only their geometry and entry angles to the SSS are different but also some of them (specially in the central region) are more convoluted near the sagittal plane. Therefore a possible influence of shearing forces in the neighbourhood of the BV-dural interface should not be dismissed. Despite constrained by the 
presence of the falx, this lateral relative movement provokes the tearing of the vessel entry into the SSS.

Such a complex problem as head injury prediction requires the analysis and reconstruction of several clinical cases with either a controlled or wellknown impact environment. Even though in this section a single case has been studied, some findings regarding the biomechanics of contusive injuries have been achieved. Additionally, the presented head model has demonstrated a predictive capability that makes it suitable for its application in impact reconstructions and it has the potential to be improved with the validation against further real-life cases.

One limitation to the current study is the use of CT images as a basis for the head model development, as the resolution is not enough to distinguish all the different intracranial structures properly. Comparing the current model to one obtained from MRI images would have provided a better validation of the results, capturing the influence of geometric fidelity of the soft tissue structures in the mechanical response of the head model. Nonetheless, such magnetic resonance images were not available, as it is not common to get both data types from one same patient. 



\section{Chapter 9}

\section{Characterisation of Non-Lethal Projectiles}

This chapter of the thesis has been developed in collaboration with the University of Strasbourg (UNISTRA-ICUBE) and with the use of their facilities.

\subsection{Background}

Non-lethal weapons (NLW) are intended to be used by the security forces mainly for crowd control, offering the capability of incapacitating individuals without resulting in severe injuries [268]. Nonetheless, since their incorporation in the 1970s, many cases of mild to severe injuries have been reported in the literature. Non-lethal projectiles (NLP) are meant to be delivered in the torso region in order to accomplish their deterrent function without inducing severe trauma [269]. However, literature reports over the last decades reveal a large number of head and face injuries due to this kind of weapons.

Millar et al.(1975)[270] provided the first report of 90 civilians injured by rubber bullets in Northern Ireland, finding that most of the injuries were located above the chest, including head and neck. Two deaths were also reported in this study. Aiming to reduce the lethality of the pioneering rubber bullets, the kinetic energy of the projectiles was reduced from 401.7 to $77 \mathrm{~J}$ [271]. However, as reported by Hiss et al.(1997)[271], ten deaths resulted from these improved rubber bullets during the Intifada, eight of them due to head impact. Instantaneous death also followed some cases where the rubber pro- 
jectile did not penetrate the skull. The impact site proved to be a determining factor for damage severity, and not only the kinetic energy of the projectile. Posteriorly, Mahajna et al.(2002)[272] gave an extensive report regarding 152 rubber bullet-injuried patients from the Israeli-Arab conflict. Approximately one third of the patients were hurt at the head, neck or face, being this group the one which presented more severe lesions with respect to the other body parts. Basilar, depressed and linear skull fractures were found in some of the subjects, but facial injuries revealed to be the most serious. When these injuries involved penetration of the rubber bullet to the brain through the eyeball the patient died, while other severe outcomes like partial or total loss of sight could result in the cases of no penetration.

In 1995, French special forces introduced the use of a new NLP, the FlashBall ${ }^{\circledR}$, a rubber ball capable of acquiring a kinetic energy around 200J. Since its incorporation, this rubber projectile has led to different injury scenarios [268, 273-275]. In a case report of 2006, Wahl et al. [268] highlighted the lack of information provided by the manufacturer of this weapon regarding its characteristics and ballistics and later, Hiquet et al.(2016)[273] suggested that a FlashBall ${ }^{\circledR}$ impact could lead to severe lesions when fired at a distance lower than $10 \mathrm{~m}$. More insight into the lessive potential of this NLP over the human head was given by Sahoo et al.(2016)[148]. They addressed the risk injury associated to FlashBall ${ }^{\circledR}$ by means of a combined method of rigid wall experiments and numerical simulations under a validated human head model. Their work reveals a good correlation between the behaviour of the projectile under the rigid wall tests and the response of the FE model. The injury assessment involved both skull fracture and DAI, showing a high probability of skull fracture for NLP velocities above $72 \mathrm{~ms}^{-1}$ and a risk of $50 \%$ or more of DAI with velocities greater than $99.2 \mathrm{~ms}^{-1}$.

In an effort to obtain a robust methodology to characterise the behaviour of NLPs, a method combining both FE modeling and experimental testing was proposed by Robbe et al.(2013)[276]. Their main purpose was to determine the safest range of engagement distances for a 40mm Nobel Sport Spartan LE-40 sponge grenade. The lower bound was determined by established thorax injury criteria in the literature [277] and the upper bound by the radius of dispersion of the projectile trajectory, which is aimed to quantify the likeliness of a shot reaching the head of the target. Skin penetration was also taken into account as a limiting parameter, but in none of the distances tested this phenomenom was observed. The method proposed consisted of five steps: (1) impact tests of 
rigid projectile against the torso of PMHS, (2) experimental impacts of flexible projectiles against rigid target, $(3,4)$ numerical recreation of the two sets of experiments and (5) coupling of the validated flexible projectile and torso models for injury measurements. The resulting optimal range of engagement distances for the tested projectile was 30 to $61 \mathrm{~m}$.

The latest work in the assessment of NLPs was performed by Oukara et al.(2017) [269]. In their study, they compared three methods for the assessment of the injury risk of NLPs using the XM1006 projectile for temporal impacts: the force wall method, experimental tests on surrogates (Ballistic Load Sensing Headforms (BLSHs)) and impact simulations on the SUFEHM [278]. The authors pointed out that maximum force on the head could be a good risk correlator, and the three methods tested proved to be suitable to predict this force value using the aforementioned projectile. However, when widening the studio adding three of the most commonly employed NLPs, the force wall method revealed some limitations, while ballistic load sensing headforms (BLSHs) and numerical simulations gave accurate results for all cases.

\subsection{Methodology}

The general purpose of this research was to assess the mechanical behaviour of some specific non-lethal projectiles under different impact conditions. This assessment is performed by means of the coupling of experimental testing and finite element modeling.

One set of experimental impact tests of a NLP against rigid wall was provided by a partnered affiliation. This set comprised impacts with various initial kinetic energies, and each single test was filmed with two high-speed cameras (for the frontal and top views, respectively). The input data from the aforementioned tests consisted of a collection of video files of the impact events and the force-time measurements recorded by a load cell attached to the rigid wall of the testing set-up. The first part of this chapter is focused on the treatment of these input data in order to obtain certain information about the experimental mechanical response of the projectile, specifically the stress-strain curves corresponding to each loading rate.

To this purpose, an in-house software for the data extraction process was implemented using Python 2.7. An overview of its working principles and the different functions it performs is given in the following section. 
The second part of the procedure consists of the development of a numerical model for the tested projectile. A 3D scan of the projectile is first performed and a mesh is afterwards obtained using the software Hypermesh. Then, the model is set under FE explicit analysis in LSDYNA using the boundary conditions from the rigid wall experimental testing. The general purpose is to achieve an adequate material law for each of the two parts of the projectile so that the overall simulated response under rigid wall impact matches the experimental data in terms of force, maximum diameter, and displacement of the rear part (all these as variables of time). Therefore, a validated numerical model of the studied projectile is achieved. This process, similar to the one followed by [148] for the validation of a FlashBall ${ }^{\circledR}$ model, is schematised in Fig.9.1.

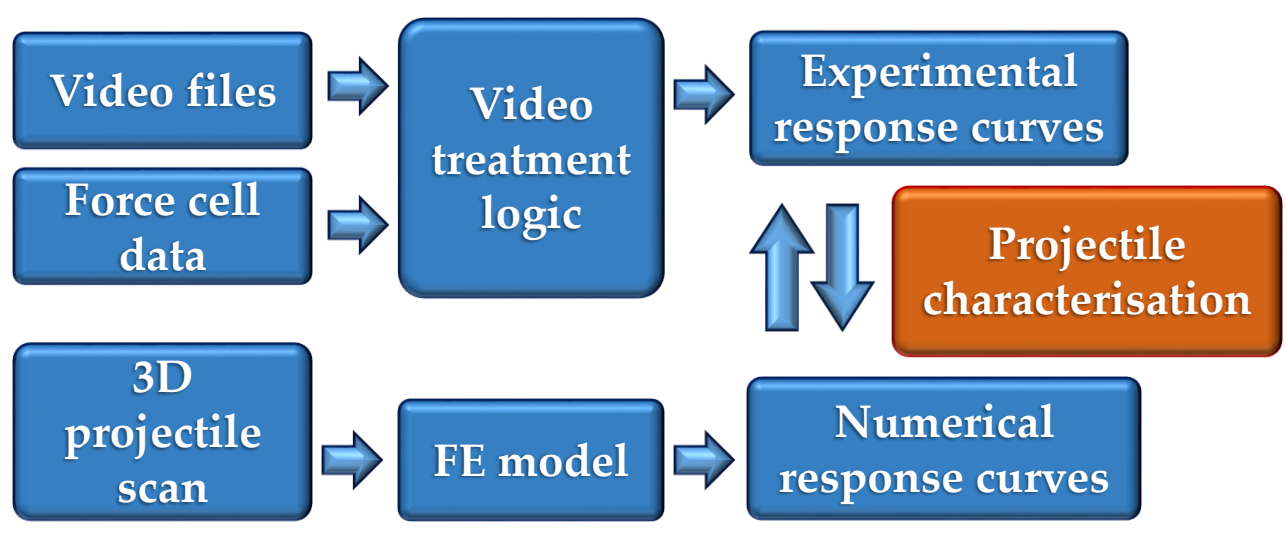

Figure 9.1: Methodology followed for the validation of a numerical NLP model

\subsection{Experimental data treatment}

The in-house developed software is intended to provide specific data from a video file. It is structured in a main interface with five tabs, each of them intended to perform one specific step of the data treatment. The intention in this section is not to deepen into the architecture of the software but gain a basic understanding of the process of data extraction, which comprises the following steps:

- Edition of the video files from experimental tests 
- Insertion of the experimental data recorded by sensors

- Tracking of specific points in the video file

- Mechanical response of the subject of study (in this case the NLP)

- Summary of the results and statistics of the recorded tests

Each of these bullet points will be briefly addressed in this section.

\subsubsection{Video edition}

Depending on the type of experiment recorded, the duration and region of interest of the video may vary, affecting its final size. Specifically, high-speed cameras are able to take a large number of frames per second, resulting in file sizes around $1 \mathrm{~GB}$. Then, the first stage of the data tracking process will consist of adapting these video files to the tracking tool itself, in order to save RAM memory and enable a faster analysis.

This pre-processing tool has three purposes: select and cut the area of the video to a certain region of interest, cut the time span of the video (as some parts may not contain relevant information) and, mainly, convert the video file to labelled ordered frames (in .png format) and save them to a folder chosen by the user. This decomposition of the video into images is the basis for the point-following process.

\subsubsection{Sensor data extraction}

The purpose of this part of the software is to introduce the force history recorded by a sensor in order to posteriorly couple it to the data extracted by the video files and therefore achieve the mechanical response of the target object. The supported file format by this platform is .tpc5, as it is the default format provided by the load cell employed in the NLP experiments considered for this study. The input signal may contain a great number of data points so it is recommended that the user limits the signal span to the event of interest to not overload the calculation algorithm. The output signal must be saved in the same folder as the video frames, so that the software will automatically extract the force data from this folder. In brief, this step of the process enables reading the sensor data and modifying the span of the signal. 


\subsubsection{Point tracking}

Once the video has been split into frames, the following stage of the process consists of the point data extraction itself. This means, automatically extracting the position of some specific points from the projectile along the whole time sequence, which will provide the necessary information to perform further calculations to obtain mechanical properties.

The logic to perform the data extraction is based on binary-image assessment employing the image edition packages from OpenCV. Our in-house application allows to perform several operations, but only the first one is mandatory: the process requires the user to set a threshold upon which the frames will be turned into black and white. The source frames are RGB format, using 8 bits per channel. To perform the binarisation, these images are turned to grayscale images, where each pixel will have a single value from 0 to 255 . The algorithm of binarisation is performed as follows: the software takes the value set by the user (from 0 to 255 ) which will define the threshold between black and white. All the pixels with a value below this threshold will be set to 0 , and all the pixels above the value will be set to 255 . This way, the resulting matrix of pixels consist of only two different values : black or white. The whole purpose of binarising the video frames is to isolate the subject of interest (in this case, the non-lethal projectile) from the background. As mentioned before, additional image edition operations can be performed over the binarised frames to improve the quality of the analysis. These options include:

- EROSION: consists of the removal of the external layer of pixels from a body. The thickness of the layer to be removed is set with the Erode slider in terms of number of pixels. This action is recommended in order to remove small regions from the background that fall outside the region of interest.

- DILATION: this operation proceeds the opposite way to the erosion. If the frame has been previously eroded, the real dimensions of the bodies of interest will have diminished. For this reason it is highly advisable to perform a dilation operation after an erosion.

- OPENING: this operation separates two areas of the same pixel value which are bonded through a small region. It may be useful to apply this operation when the tracking process involve two bodies getting into contact. The presence of shadows between the two bodies that are close 
to each other may give a false positive in the recording of the contact surface.

- CLOSING: the complementary opperation to opening. Two regions separated in the image become merged if the distance between them is lower than the number of pixels set by means of the Close slider.

However, some images may require an additional treatment, especially when dealing with high-speed impact videos. To overcome this problem, the user can manually edit single frames. This option can be useful to remove noisy surfaces near the rigid wall or the front surface of the projectile.
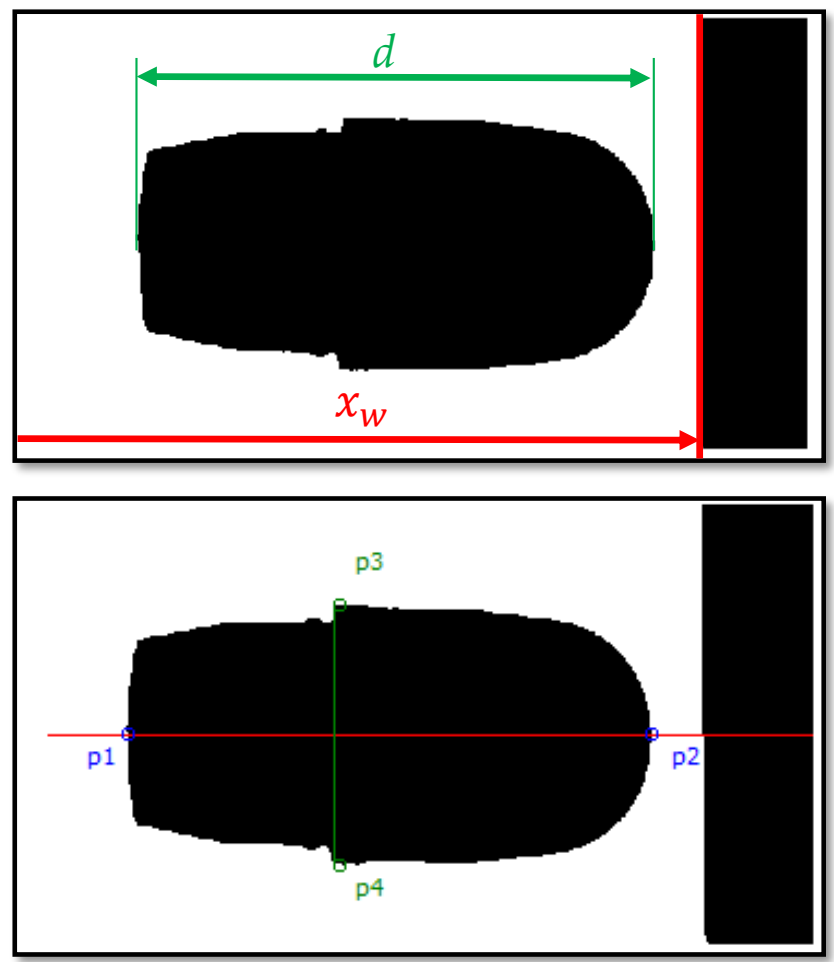

Figure 9.2: Cut view of the point tracking tab after the computation of the contact diameter (red) between the projectile and the wall.

An indispensable step that can be taken either after or before the frame 
binarisation is the calibration. It means to set a determined distance of known magnitude so the software can compute the pixel-to-length ratio and get the displacement data in the adequate unit system. The user is required to select four points in the image: the first two will define a certain known distance (e.g. the diameter of the projectile or the rigid plate) and the latest two will define the position of the rigid plate's surface, as can be seen in Fig. 9.2 (top). After that, the tool queries the user to type the magnitude of the distance between the two first points, and then select the units of this metric.

After image adaption and calibration the algorithm is prepared to perform the point detection process. A line of reference is required by the algorithm to initialise the tracking process. The user should set this "line of interest" in such way that it includes the points whose displacement is desired to track. In the case we are studying, a good choice would be to make this line of interest coincide with the longitudinal midplane of the projectile. This way, the problem size has been reduced to a single dimension, as the iteration will be performed only along the line of interest. After that, the software records the position of the rear and the frontal points of the projectile, both located on the midline at each frame. Furthermore, the tool identifies the highest and lowest points of the projectile in the plane of the frame, defining its maximum cross length. These four points are displayed in the frame canvas: the rear and frontal points are identified in blue with labels p1 and p2, respectively, and the top and bottom points are highlighted in green, as well as the maximum diameter (as can be seen in Fig 9.2 (bottom)).

Another relevant fuction of this algorithm is the recording of the contact event between the projectile and the wall. The tool already knows the location of the wall as it was defined in the calibration step and the contact tracking operation can be performed once the four principal points have been tracked. The contact region, which is indeed a straight line, is displayed as a red line painted on the canvas (Fig 9.3). It is advisable to check on each frame that the contact diameter given by the software corresponds to the actual projectile and is not altered because of the presence of shadows. If the binarisation process has been adequately performed, there should not be problems with the values given by the platform. However, if the wall position has not been properly defined, the user can re-calibrate the images and only repeat the tracking process. 


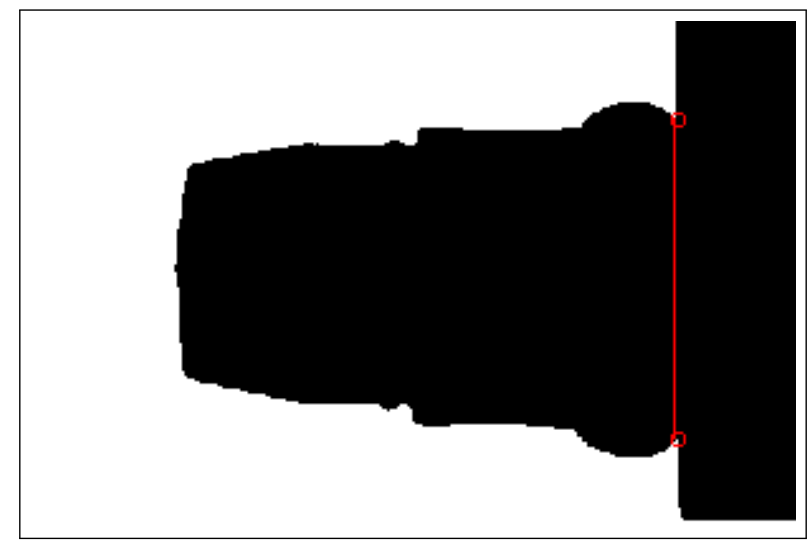

Figure 9.3: Automatic detection of the contact diameter (red) between the projectile and the wall.

\subsubsection{Mechanical response of the projectile}

The last stage of the experimental data postprocessing is the derivation of a mechanical behaviour law for the tested projectile. This is achieved by coupling the previously treated data (obtained from point detection and the force signal). For the completion of the process, the tool will query the user to enter the frame rate of the video camera that made the recording of the test (in fps) in order to compute the corresponding time scale. The rest of the window, provides the following information:

- Velocity and acceleration of the test subject, both as a function of time.

- Maximum diameter and contact diameter as a function of time.

- Force history and stress-strain curves.

When saving the results from this window, a multiarray file with the structure depicted in Fig.9.4 is stored for further data analysis. 


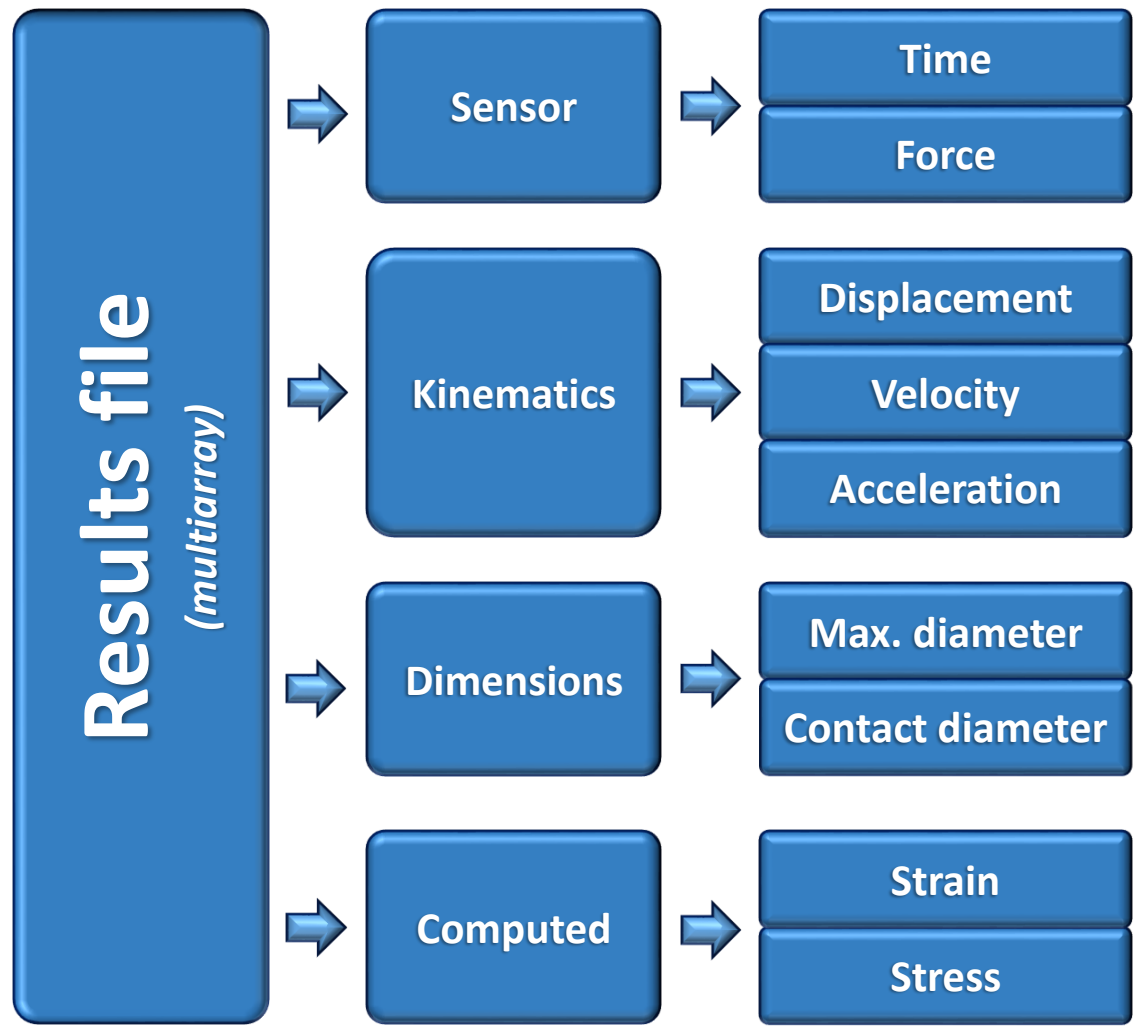

Figure 9.4: Structure of the data stored from each experimental test in the Mechanical response tab.

\subsubsection{Results and statistics}

This last stage of the data extraction process aims to visualise the main data obtained on the experimental testing and perform a basic statistical analysis on them.

The mean and standard deviation of the different variables (stress-strain, force and contact surface) are calculated and stored in the form of mean plus upper and lower corridors. Finally, there is the option to store the data from each of the displayed tests and their statistics in an .xls file. The data will be arranged on the output spreadsheet as follows: 
- Time (ms)

- Projectile velocity $\left(\mathrm{ms}^{-1}\right)$

- Acceleration of the projectile $\left(\mathrm{ms}^{-2}\right)$

- Maximum cross area of the projectile $\left(\mathrm{mm}^{2}\right)$

- Contact time (ms)

- Contact surface between the projectile and the wall, estimated as a circle $\left(\mathrm{mm}^{2}\right)$

- Strain (dimensionless) - Stress (MPa)

- Time measured by the load cell (ms)

- Force $(\mathrm{N})$

\subsection{Numerical modeling}

A finite element (FE) model of the employed non-lethal projectile is developed based on the topology obtained by a 3D scan of one of these specimen. This geometrical data (consisting of a tetrahedral mesh) acts as the input for the meshing software Hypermesh. Combining the information from the scan with the actual dimensions of the projectile, an accurate geometry is obtained. Then, a quarter the body is meshed using hexahedral elements, and the resulting mesh is projected in order to ensure symmetry with respect to the longitudinal axis of the projectile. The average element size is $2 \mathrm{~mm}$, resulting in a total of 5040 elements and 6864 nodes.

The mesh is divided in the two components of the projectile: the plastic cage and the foam/rubber head. However, these two parts share their common nodes in order to ensure a continuous mesh that will ease the simulation process. Both meshed parts are depicted in Fig.9.5.

The projectile mesh is imported to LS-DYNA[279] in order to perform explicit FE simulations to recreate the experimental rigid wall tests performed. To this purpose, the LS-DYNA option *RIGID_WALL is employed, thus creating a $37.5 \mathrm{~mm}$ radius cylinder with $5 \mathrm{~mm}$ depth. A friction coefficient of 0.1 is set between the wall and the projectile. This dynamic problem will be defined by setting a *INITIAL_VELOCITY_GENERATION card on the projectile mesh. The velocity will be defined in the longitudinal axis of the projectile, that is perpendicular to the rigid plate surface, and its magnitude will be the same as the one from each of the experimental tests: 56, 70 and $80 \mathrm{~ms}^{-1}$, respectively. The density of each of the two material parts is set in 
such way that the resulting projectile mass is $62.8 \mathrm{~g}$ and the center of mass falls in a reasonable position, as represented in Fig 9.6.
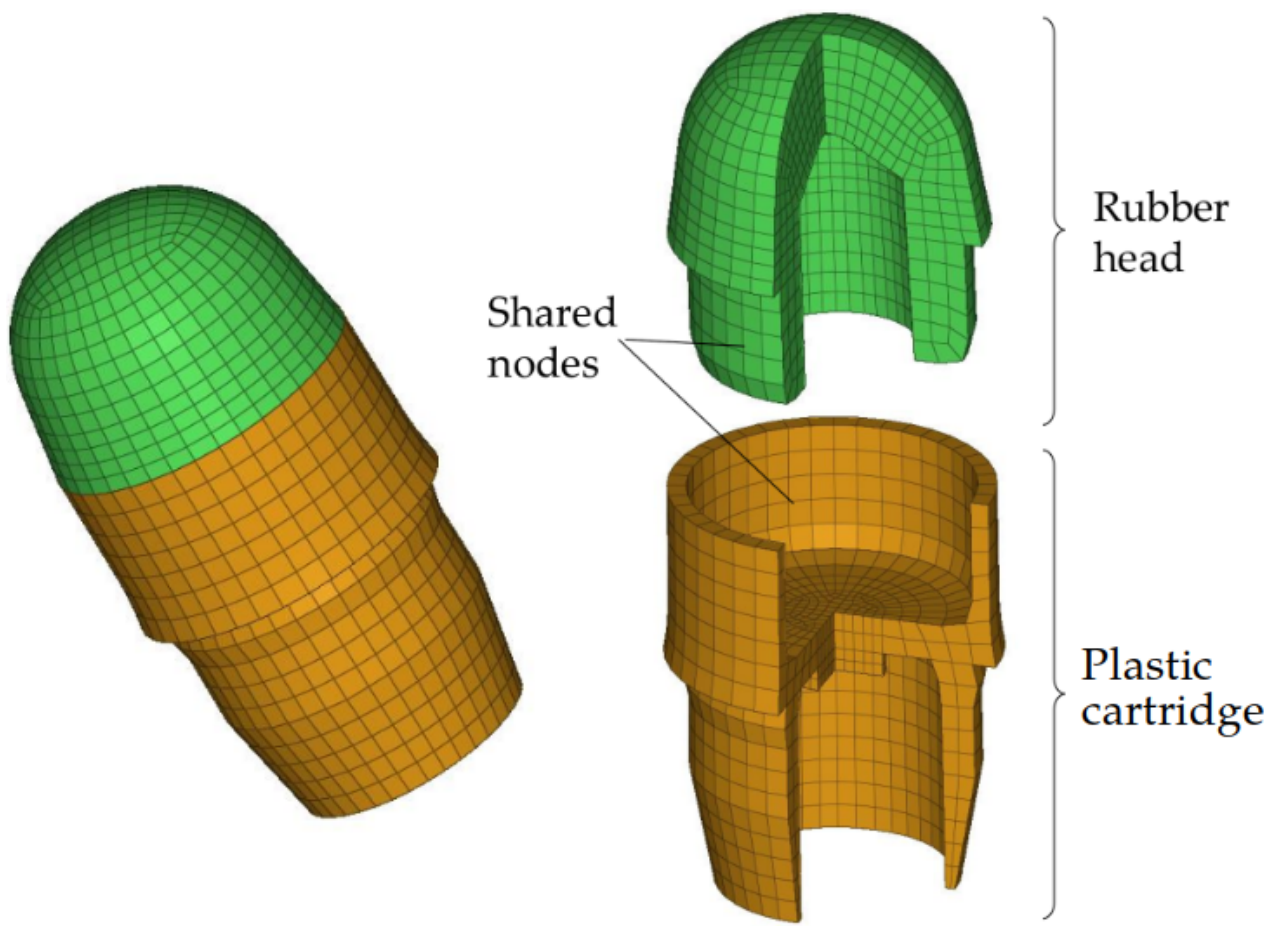

Figure 9.5: Projectile hexahedral mesh obtained by means of Hypermesh.

During the simulations, the hourglass energy was controlled to ensure reliable results. LS-DYNA provides different options for hourglass assessment [280]. Between them, the most suitable for the problem here studied is the Belytschko-Binderman [157] assumed strain co-rotational stiffness form. This formulation works properly when dealing with soft materials, avoiding the subsequent hourglass modes without involving a stiffening of the response (a value of 0.1 was chosen for the hourglass coefficient).

The contact formulation *CONTACT_AUTOMATIC_SINGLE_SURFACE is applied to the model, as it is normally recommended for impact analysis. The contact stiffness calculation is set to the soft-constraint based approach, 
which is suitable for modeling the interaction between materials with substantially different stiffnesses.

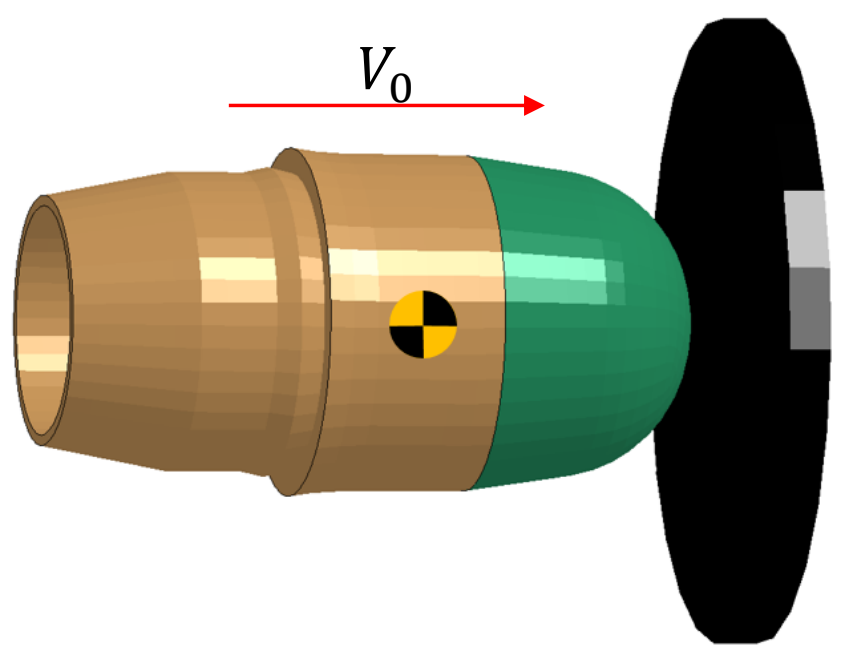

Figure 9.6: LS-DYNA assembly for the simulation of impact against rigid wall.

Regarding the applied material properties, a parametric study is performed in order to set the most suitable constitutive law for each of the components, being the foam head the most critical to determine. The validation of the projectile numerical model can therefore be achieved.

\section{$9.5 \quad$ Results}

After the simulations of impact against rigid wall at the three different initial velocities of the projectile, some of the results are collected and summarised in Figures 9.7, 9.8 and 9.9. Each of the plots shows both the experimental response obtained from the data processing described in Section 9.3 and the numerical aproach given by LS-DYNA.

Three variables have been chosen to validate the response of the numerical model: the time evolution of the projectile maximum diameter, the displacement along time of the rear point of the projectile and the force measured by 
the rigid wall load cell. For the first two parameters, the numerically obtained curve falls within the range given by the experimental tests for the three initial kinetic energy values. The validation of the rigid wall force is more complex, due to the contact interaction between the projectile head (soft) and the modeled rigid plate (its stiffness tends to infinity). In fact, the simulated contact force histories reveal a sharp increase at the beginning of the impact that is followed of a change in slope with high-frequency oscillations until the peak value is reached. However, the maximum experimental value of the force has been properly predicted for all three cases, and the time span of the force pulse is quite similar to the one given by the sensor. Given the smoothness of the experimental curves from the load cell, it is plausible that a previous signal conditioning (filtering) had been applied during the acquisition and therefore removing noise.
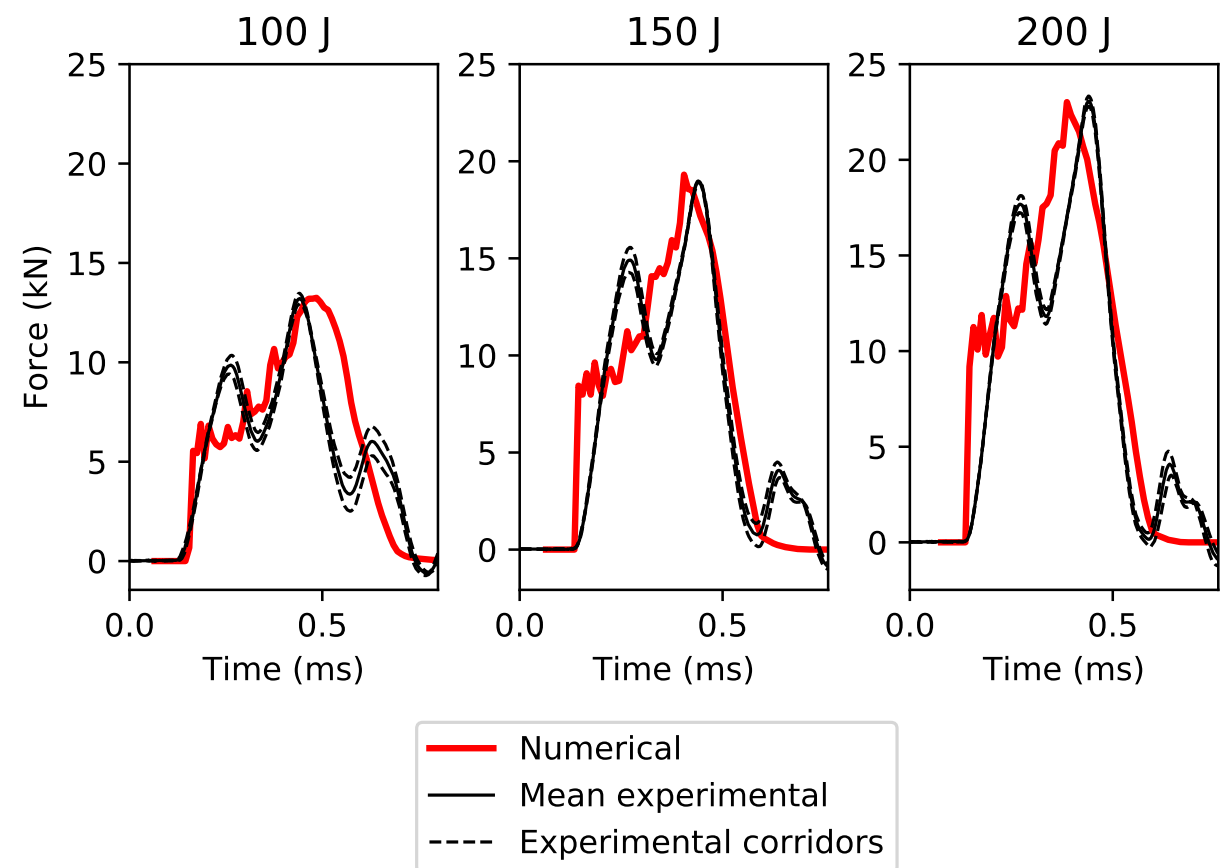

Figure 9.7: Impact force measured at the rigid wall for the three initial kinetic energies tested. Mean experimental curves in solid black line, upper and lower experimental corridors in dashed line, and numerical response in red. 
In all plots, the mean and upper corridors from the experimental response are plotted. The width of the confidence interval is considerably low, especially for the displacement of the rear part of the projectile. This fact suggests a high consistency and repeatability in the experimental results (within the five impacts analysed for each kinetic energy level), which is even higher in the cases of greater initial kinetic energy.

The most critical parameter during the video post-processing is the maximum diameter of the projectile. This finding is reasonable given that this magnitude is very sensitive to changes in the incidence and rebound angles. This same issue applies for the surface in contact with the rigid wall. Results displayed in this section are based on the assumption of a circular contact area. Prior analyses were performed employing the tracked responses from the two high-speed cameras (one placed at one side of the rigid wall and the other pointing to the top of it) and assuming an elliptical contact surface. Nonetheless, data from the second camera were not robust enough as revealed a great dispersion between similar impacts. For this reason, these data were omitted for further calculations.
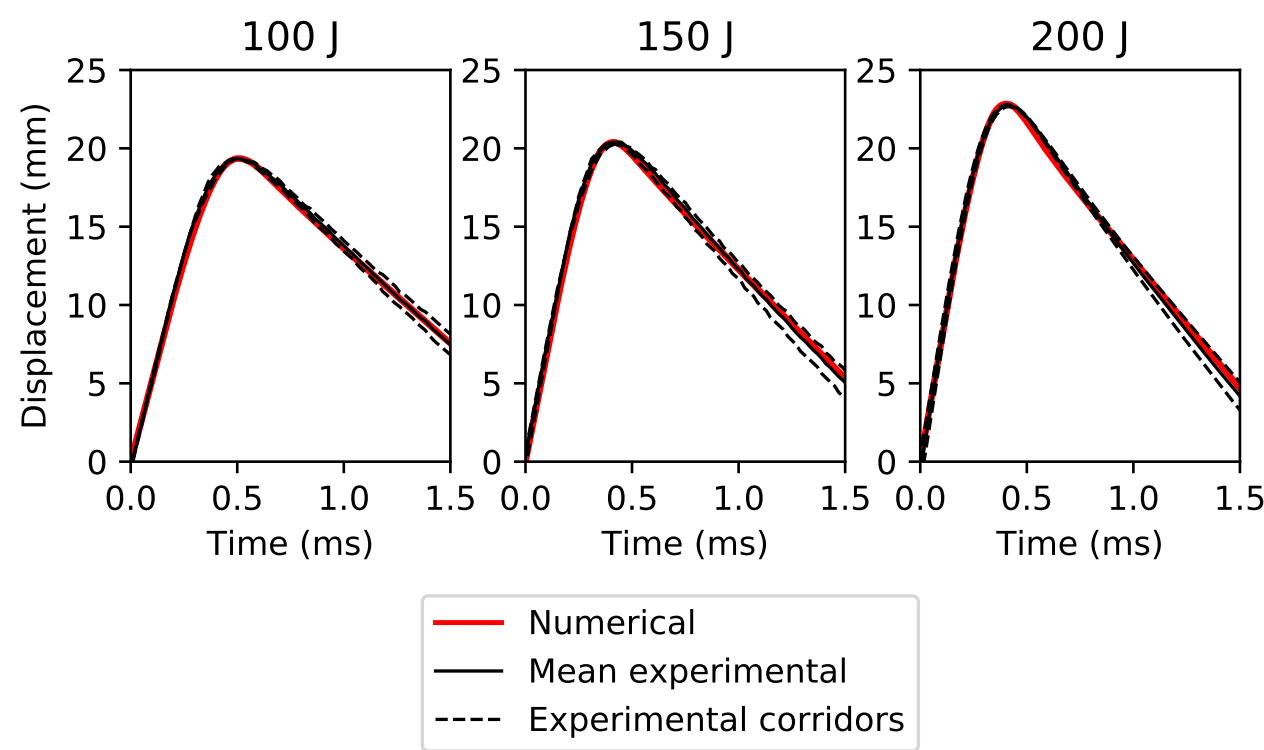

Figure 9.8: Comparison between the simulated and experimental displacements at the rear of the projectile. 

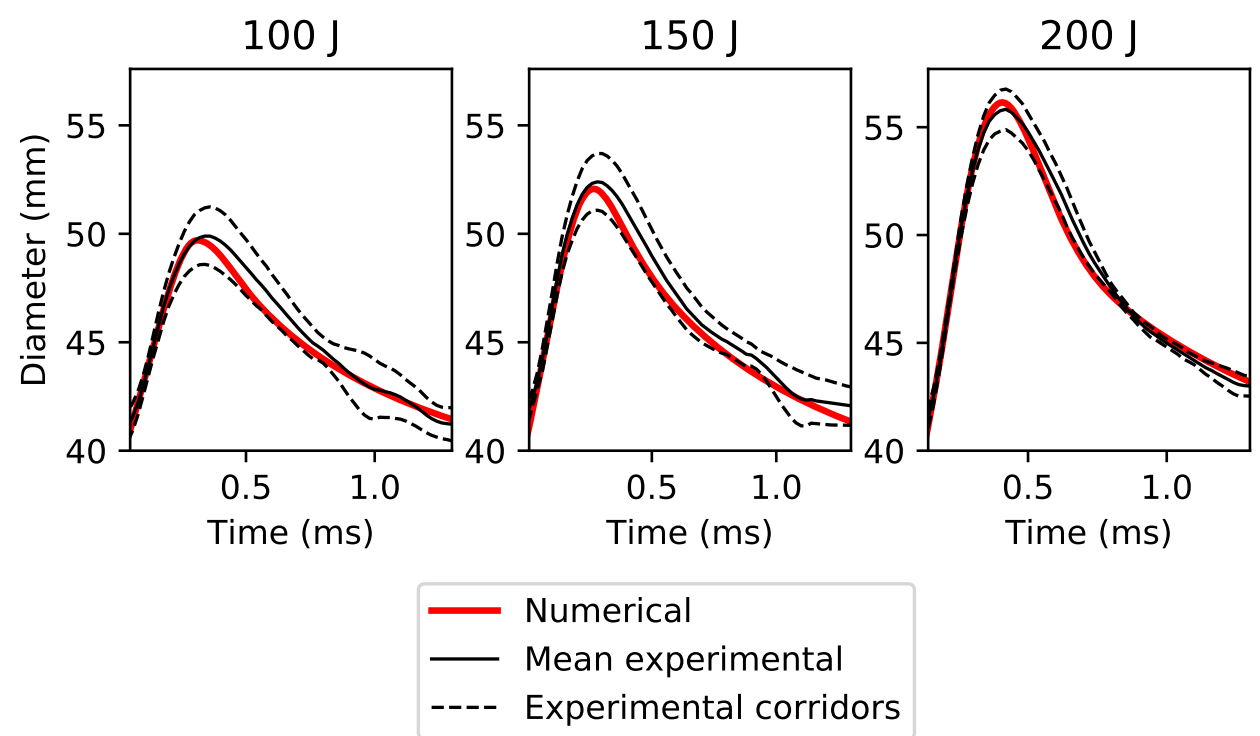

Figure 9.9: Maximum diameter of the projectile during the impact event calculated experimentally and numerically.

From the data extracted from the experimental test videos, stress-strain curves for the projectile were derived based on the load cell force and the computed contact surface. However, the choice of a material law able to match the experimental response was not straightforward, which means that the actual amount of useful data derived from the rigid wall tests is not sufficient to fully characterise the projectile behaviour. In fact, direct implementation of the experimental stress-strain curves into a material law was tested, giving as a result a considerably stiff response far from the soft behaviour of the rubber head of the projectile.

Several material laws were tested under LS-DYNA to model the response of the projectile head, including both foam and rubber material formulations. The most accurate response was achieved applying the LS-DYNA built-in material model *MAT_SIMPLIFIED_RUBBER/FOAM [281] (corresponding to the results shown in Fig.9.7, Fig.9.8 and Fig.9.9) in which a user-specified stress-strain curve was included. A first approach was to maintain this same stress-strain curve for the three different initial kinetic energy cases. Nonetheless, the behaviour of the projectile's head proved to be rate-dependent, therefore having to perform an interpolation between different stress-strain curves 
validated at different strain rates. Furthermore, the behaviour during unloading proved to be also rate-dependent, and its characterisation was fundamental in order to recreate the projectile recovery of its initial shape after the impact.
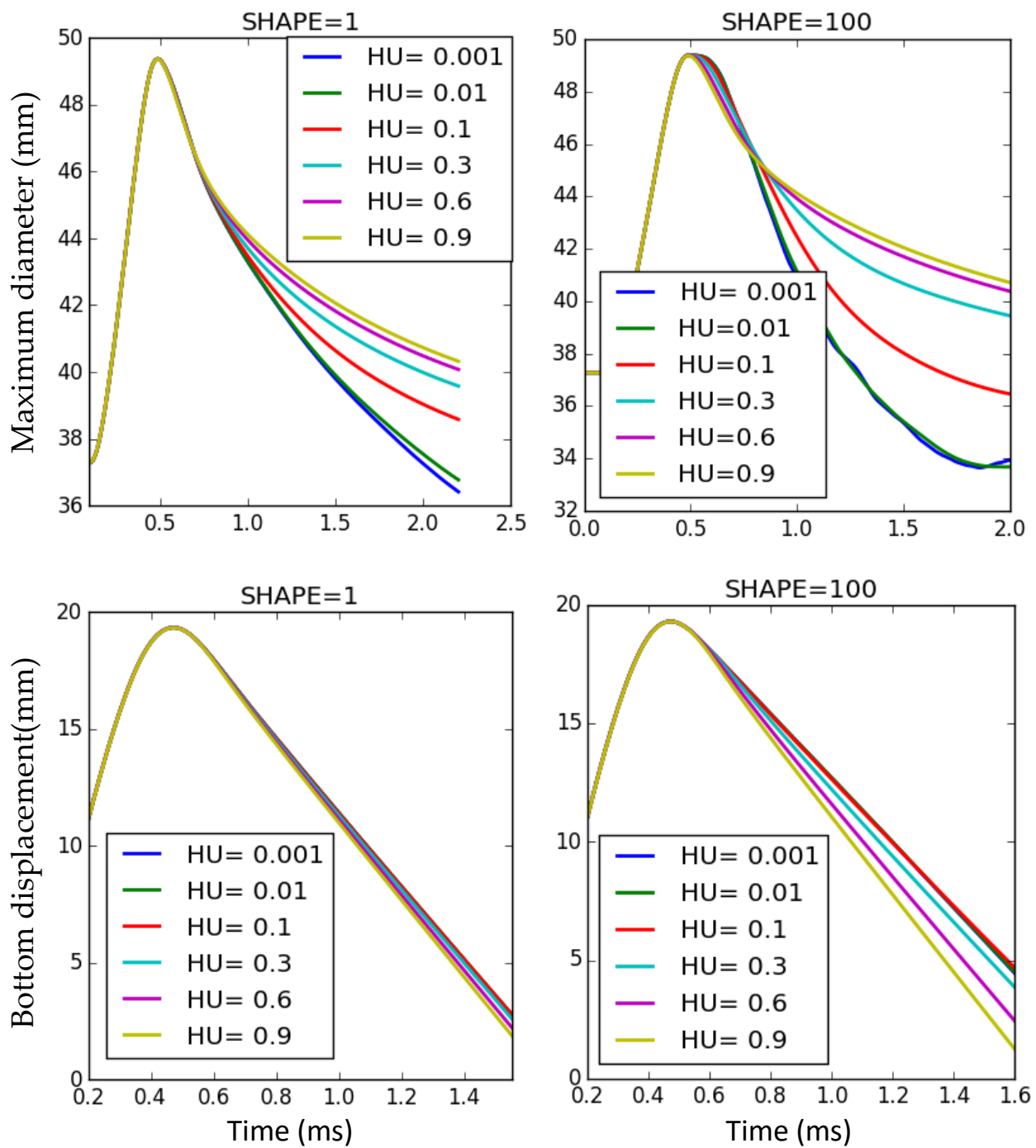

Figure 9.10: Influence on the unloading parameters on the maximum diameter and bottom displacement of the projectile. 
The plastic part of the projectile, which is often assumed to behave as a rigid material, proved to have influence on the overall projectile response, especially when increasing impact velocity, as certain deformation in the radial direction appears in the junction with the rubber/foam head. Therefore, a deformable material law is set, whose material parameters affect the whole projectile validation. A compromise between both material models needs to be reached to match the experimental curves.

Particularly, there are two parameters implementable in LS-DYNA that modify the behaviour of rubber-like materials during unloading. These parameters, the hysteretic unloading factor (HU) and SHAPE define automatically the unloading path and make it similar to the one characteristic of low density foams. Fig. 9.10 illustrate the influence of these two parameters on the maximum diameter and rear projectile displacement during the rebound event. As the $\mathrm{HU}$ factor approaches 0 , the unloading response resembles the path of the loading one and reducing the hysteresis.

All the previously mentioned factors highlight the need of a deeper insight into the characterisation of this kind of weapons, as it has been proved that their behaviour is not easily predictable. Each new NLP should undergo a complete experimental and numerical characterisation before being released to the market. Special care should be taken to account for the whole range of possible kinetic energies in order to stablish an adequate determination of the injury potential.

With the validation of the projectile model in terms of its maximum diameter and the displacement of its bottom part, the deformation history of the NLP along the impact duration is recreated. If it is coupled with the prediction of the rigid wall peak force, it makes the numerical model suitable for further applications, like injury prediction if combined with biofidelic numerical models $[148,269]$.

\subsection{Conclusions}

In this part of the Thesis, a method for characterising the impact behaviour of a specific non-lethal projectile has been developed. The method is based on experimental tests of impact against a rigid wall recorded by high-speed cameras which are employed for the validation of a numerical FE model of the projectile. This validation process enables the extraction of proper material 
properties for the projectile constituents to recreate its mechanical behaviour under certain impact conditions.

An in-house tool for data extraction from high-speed videos has been developed in order to compute some parameters relevant for the validation of a NLP numerical model: maximum projectile diameter along time and displacement of the projectile rear point. Adding the force signal from the load cell, a proper characterisation of a numerical projectile model has been performed for different impact velocities. It has been demonstrated that the choice of a material law for the NLP is not straightforward as it cannot be directly derived from the stress-strain curves achieved experimentally. Care needs to be taken in order to ensure a good matching between the numerical and experimental results.

Rigid wall impact testing appears as an adequate tool to perform the numerical validation of projectile models, being this the starting step for further risk assessment. However, the rigid wall method itself appears inaccurate to directly make injury risk predictions unless specific experimental tests on PMHS had been previously performed with the same projectile.

Therefore, this characterisation study, enhanced by the development of a mechanical data extraction program from video files, can be considered as a part of a wider process to achieve an accurate injury risk assessment of non-lethal weapons. 



\section{Chapter 10}

\section{Conclusions and future works}

Art is never finished, only abandoned.

— Leonardo da Vinci

The main results and findings achieved along this Thesis can be summarised in the following bullet points:

- Impact behaviour of protective composite materials has proved to be highly dependent on the input energy as different damage mechanisms may be triggered. Proper numerical modeling of this behaviour has been achieved by means of the application of the Cumulative Damage Mechanics (CDM) theory for homogenised elements. Discretisation of the material along its thickness in different plies enables the implementation of cohesive laws at their interfaces. Delamination damage has been identified as the first mechanism to arise in the energy-dissipation process, and the only one present in the cases of low impact energies (around $24 \mathrm{~J})$. As the kinetic energy of the impacting body increases, matrix damage and fiber breakage become predominant in impact attenuation.

- A numerical human head model has been developed from CT images of a real subject. By implementing a proper set of material properties for the different head tissues it has been possible to reconstruct the experimental response from different impact tests on post-mortem human subjects 
(PMHS) reported in the literature. Some phenomena such as skull fracture or intracranial lesions can be predicted following the methodology presented in Chapter 5, as it has been demonstrated by means of the recreation of ballistic tests from Sarron et al. [222].

- By means of ballistic impact simulations on a helmeted head the injuries associated to the testing standard NIJ-STD-0106.01 have been quantified. Despite being no trace of neuronal damage or risk of skull fracture, according to the head injury criteria the intracranial pressures sustained during this acceptance protocol exceed the thresholds for severe injury both for coup and countrecoup lesions. This finding suggests that current testing protocols should take into account more variables than just the BFD and maximum acceleration of the head, which are not explicitly related to head injury biomechanics. In view to the results obtained in Chapter 6, the use of dummies instrumented with pressure gauges in helmet acceptance procedures is highly encouraged.

- Some helmet manufacturers only provide a single composite shell with fixed dimensions that is adapted to the different head sizes of the users by means of adjusting the padding thickness. It has been demonstrated through numerical simulation that, as the separation between head and helmet shell decreases, the injury risk dramatically increases even though the BFD values remain far less than the limit proposed by the standards.

- One concern in the industry of personal protections is weight reduction, which can be achieved by means of lighter materials or more efficient architectures. Several stack configurations of a helmet composite shell have been studied in this Thesis against ballistic frontal impact. From the configurations studied the best performance in terms of injury risk avoidance and BFD reduction consisted of 16 layers of woven aramid alternatedly stacked at $0^{\circ} / 90^{\circ}$ and $45^{\circ} /-45^{\circ}$. If a similar protection level is desired with an improved resistance to penetration, it is suggested to reinforce the woven-aramid shell with two woven layers of CFRP at the striking surface. This addition of $120 \mathrm{~g}$ in mass leads to a $10 \%$ increase in the energy absorption of the shell.

- Ultra-high molecular weight polyethylene (UHMWPE), which is increasingly being introduced in the armour industry due to its low weight and 
high strength, has been assessed in Chapter 7 under ballistic impact conforming a helmet shell with different stack configurations. Results show that unidirectional lay-ups of this material do not provide enough resistance to penetration, whereas cross-ply architectures (like the commercial Dyneema ${ }^{\circledR}$ HB26) improve the energy absorption but result in great deformations at the impact site, sufficient to penetrate the head of the user. Hybridisation of cross-ply UHMWPE layers with wovenaramid plies reduces this effect but introduces a penalty in the overall shell weight.

- A detailed human head numerical model has been developed and validated against cadaveric experiments of intracranial motion from Hardy et al. [256]. The model is employed for the reconstruction of a real clinical case involving a fall. Assuming the head model is capable to predict skull fracture with enough accuracy, the impact environment can be recreated by means of the reconstruction of the cranial fracture patterns present in the clinical history.

- An effort has been made to establish a mechanical predictor for subdural hematoma (SDH). Complexity of the vascular system and its interspecimen geometrical variations affect the incidence and location of this injury, making it difficult to predict with a single head model. By means of the reconstruction of experimental tests on acute SDH from Depreitere [137], an ultimate stress threshold of 3.5 $\mathrm{MPa}$ has been chosen as adequate to mimic the rupture of bridging veins.

- Non-lethal weapons are a potential threat capable of causing severe head injuries. A characterisation study of a specific non-lethal projectile (NLP) has been performed from experimental impact tests against a rigid wall. Afterwards, numerical modeling of the impact conditions in the experiments have enhanced the establishment of a proper material law for the projectile, which could be employed for further simulations to predict the impact injury risk on a head model struck by this kind of threats.

All these findings, despite clarifying certain aspects, also lead to new unknowns. For this reason, these could be considered the starting point for new future studies at the short and long range: 
- It would be desirable to conduct experimental tests on skull bone samples or whole calvaria in order to create a controlled database that could be employed for the validation of skull numerical models. Given the ethical limitations when performing experiments on PMHS, cranial samples from other species with similar properties could be studied. Another feasible option is the creation of surrogate materials for the different tissues of the human head and generate an instrumented dummy whose impact behaviour resembles the one of a living body.

- Comparison of the experimental response of a helmeted Hybrid III dummy with the numerical prediction on the in-house developed head model in order to address the deficiencies and reliability of this kind of testing devices. As it has been discussed in Chapter 6 from this Thesis, acceleration-based criteria may not capture the whole mechanical response of an impacted head, as direct contact loads that may cause skull fracture are not taken into account.

- Incorporation of other ammunition or threat typologies in order to assess the protection of ballistic helmets from a more general perspective.

- Perform ballistic tests on different composite materials, either alone or hybridised, in order to characterise their mechanical response to highvelocity impact loading without the need of relying on data reported in the literature. Once having validated models, they could be implemented into the geometry of a helmet shell covering a head model and perform ballistic simulations of the assembly. This methodology would speed up the design process of personal protections as the construction of some prototypes can be substituted by their numerical surrogate.

- Creation of a database of clinical cases of head trauma derived from impact situations. If accompanied by CT scans and detailed diagnosis, accidental reconstruction by means of FEA is possible and, if the number of studied cases is large enough, new injury tolerances and criteria can be developed.

- The methodology of employing human head FE models under different impact scenarios poses a great interest in the field of clinical procedures. 
Coupling the results of FE simulations with other methodologies such as machine learning can help to speed-up the diagnosis procedure, allowing to get specific results according to the patient's head morphology and geometry. If a large enough set of impact simulations employing different boundary conditions and head geometries is obtained, rapid diagnosis could be achieved by means of direct extrapolation using the patient's specific features. This methodology would therefore remove the need of performing the FE analysis each time. Other factors such as age or gender could also be taken into account by deriving statistical relations between these parameters and their effect on the mechanical response of the head.

- Impact simulation of the developed NLP against the human head model in order to verify the non-lethality of this type of threats. 


\section{Diffusion of the results}

Some of the results achieved in this Thesis have been published in scientific journals and presented in conferences:

\section{$\underline{\text { Scientific journals }}$}

Lozano-Mínguez, E., Palomar M., Infante D., Rupérez M. J., Giner E., Assessment of mechanical properties of human head tissues for trauma modelling, International Journal for Numerical Methods in Biomedical Engineering, 2018, $34(5)$.

Palomar M., Lozano-Mínguez E., Rodríguez-Millán M., Miguélez M. H., Giner E., Relevant factors in the design of composite ballistic helmets, Composite Structures, 2018, 201:49-61.

Palomar M., Belda R., Giner E., Effect of different helmet shell configurations on the protection against head trauma, Journal of Strain Analysis for Engineering Design, 2019, pages 1-8.

\section{International Conferences}

Palomar M., Lozano-Mínguez E., Rodríguez-Millán M., Miguélez M. H., Giner E., Assessment of injury criteria through a human head model wearing an advanced combat helmet, 23rd Congress of the European Society of Biomechanics (ESB 2017) Sevilla, Spain.

Palomar M., Lozano-Mínguez E., Rodríguez-Millán M., Miguélez M. H., Giner E., Influence of the stack composition of composite combat helmets on the ballistic trauma, 4th International Conference on Mechanics of Composites (MECHCOMP 2018), Madrid, Spain.

Palomar M., Lozano-Mínguez E., Rodríguez-Millán M., Miguélez M. H., Giner E., Effect of head size over the performance of an Advanced Combat Helmet, 8th World Congress of Biomechanics (2018, Dublin, Ireland)

Palomar M., Lozano-Mínguez E., Belda R., Giner E., Effect of the stack configuration of a composite helmet shell on head trauma, 25th Congress of the European Society of Biomechanics (2019, Vienna, Austria). 


\section{National Conferences}

Lozano-Mínguez, E., Palomar M., Infante D., Rupérez M. J., Giner E., Comparison of mechanical properties of human head tissues through a finite element model, VI Reunión del Capítulo Nacional Español de la Sociedad Europea de Biomecánica (2016, Badajoz, Spain), ISBN: 978-84-697-6124-3.

Lozano-Mínguez, E., Palomar M., Infante D., Rupérez M. J., Giner E., Comparison of the biomechanical fracture thresholds of human head tissues through a finite element model, XXXIV Encuentro del Grupo Español de Fractura (2017, Santander, Spain) ISBN: 0213-3725, pages 204-210.

Palomar M., Lozano-Mínguez E., Giner E., Efecto de la configuración de la calota de un casco de combate sobre el trauma encefálico en impacto balístico, VIII Reunión del Capítulo Nacional Español de la Sociedad Europea de Biomecánica (2018, Castellón, Spain), ISBN: 978-84-09-06674-2, pages 85-86. 



\section{Acknowledgements}

The author would like to thank the financial support received from the Spanish Ministry of Economy and Competitiveness and the FEDER program through the project RTC-2015-3887-8, and from the Regional Ministry of Education, Research, Culture and Sport of the Generalitat Valenciana through the program PROMETEO 2016/007. 


\section{Bibliography}

[1] F. G. Evans and H. R. Lissner. Tensile and Compressive Strength of Human Parietal Bone. Journal of Applied Physiology, 10(3):493-497, 1957.

[2] D. H. Robbins and J. L. Wood. Determination of mechanical properties of the bones of the skull. Experimental Mechanics, 9(5):236-240, 1969.

[3] J. H. McElhaney, J. L. Fogle, J. W. Melvin, R. R. Haynes, V. L. Roberts, and N. M. Alem. Mechanical properties of cranial bone. Journal of Biomechanics, 3(5):497-511, 1970.

[4] W. T. Dempster. Correlation of types of cortical grain structure with architectural features of the human skull. American Journal of Anatomy, 120(1):7-31, 1967.

[5] R. P. Hubbard. Flexure of layered cranial bone. Journal of Biomechanics, 4(4):251-263, 1971.

[6] J. L. Wood. Dynamic response of human cranial bone. Journal of Biomechanics, 4(1):3-12, 1971.

[7] R. Delille, D. Lesueur, P. Potier, P. Drazetic, and E. Markiewicz. Experimental study of the bone behaviour of the human skull bone for the development of a physical head model. International Journal of Crashworthiness, 12(2):101-108, 2007.

[8] J. A. Motherway, P. Verschueren, G. Van Der Van Der Perre, J. Vander Sloten, and M. D. Gilchrist. The mechanical properties of cranial bone : The effect of loading rate and cranial sampling position. Journal of Biomechanics, 42(13):2129-2135, 2009. 
[9] A. Auperrin, R. Delille, D. Lesueur, K. Bruyère, C. Masson, and P. Drazétic. Geometrical and material parameters to assess the macroscopic mechanical behaviour of fresh cranial bone samples. Journal of Biomechanics, 47(5):1180-1185, 2014.

[10] S. Boruah, D. L. Subit, G. R. Paskoff, B. S. Shender, J. R. Crandall, and R. S. Salzar. Influence of bone microstructure on the mechanical properties of skull cortical bone: A combined experimental and computational approach. Journal of the Mechanical Behavior of Biomedical Materials, 65(May 2016):688-704, 2017.

[11] E. S. Gurdjian, H. R. Lissner, and J. E. Webster. Mechanism of production of linear skull fracture: further studies on deformation of skull by "stresscoat" techique. Surg., Gynec. E Obst., 85:195-210, 1947.

[12] E.S. Gurdjian and H.R. Lissner. Deformations of the skull in head injury as studied by the stresscoat technic. The American Journal of Surgery, 73(2):269-281, feb 1947.

[13] E. S. Gurdjian, J. E. Webster, and H. R. Lissner. Studies on skull fracture with particular reference to engineering factors. The American Journal of Surgery, 78(5):736-742, 1949.

[14] E. S. Gurdjian, J. E. Webster, and H. R. Lissner. The Mechanism of Skull Fracture. Radiology, 54(3):319-339, 1950.

[15] A. M. Nahum, J. D. Gatts, C. W. Gadd, and J. Danforth. Impact tolerance of the face and skull. Proceedings of Twelfth Stapp Car Crash Conference, 1968.

[16] V. R. Hodgson, J. Brinn, L. M. Thomas, and S. W. Greenberg. Fracture behavior of the skull frontal bone against cylindrical surfaces. Proceedings of Fourteenth Stapp Car Crash Conference, 1970.

[17] J. W. Melvin and F. G. Evans. A strain energy approach to the mechanics of skull fracture. In 15th Stapp Car Crash Conference, 1971.

[18] J. H. McElhaney, R. H. Hopper, R. W. Nightingale, and B. S. Myers. Mechanisms of Basilar Skull Fracture. Journal of neurotrauma, 12(4):669-678, 1995. 
[19] D. L. Allsop, T. R. Perl, and C. Y. Warner. Force/deflection and fracture characteristics of the temporo-parietal region of the human head. In Stapp Car Crash Conference, 1991.

[20] D. L. Allsop, C. Y. Warner, M. G. Wille, D. C. Schneider, and A. M. Nahum. Facial impact response - a comparison of the Hybrid III dummy and human cadaver. SAE Transactions, 97(4):1224-1240, 1988.

[21] N. Yoganandan, F. A. Pintar, A. Sances, P. R. Walsh, C. L. Ewing, D. J. Thomas, and R. G. Snyder. Biomechanics of skull fracture. Journal of neurotrauma, 12(4):659-668, 1995.

[22] H. Delye, P. Verschueren, B. Depreitere, I. Verpoest, D. Berckmans, J. Vander Sloten, G. Van Der Perre, and J. Goffin. Biomechanics of frontal skull fracture. Journal of Neurotrauma, 24(10):1576-1586, 2007.

[23] P. Verschueren, H. Delye, B. Depreitere, C. Van Lierde, B. Haex, D. Berckmans, I. Verpoest, J. Goffin, J. Vander Sloten, and G. Van der Perre. A new test set-up for skull fracture characterisation. Journal of Biomechanics, 40(15):3389-3396, 2007.

[24] J. Cormier, S. Manoogian, J. Bisplinghoff, S. Rowson, A. Santago, C. McNally, S. Duma, and J. Bolte. The tolerance of the frontal bone to blunt impact. Journal of Biomechanical Engineering, 133(2), 2011.

[25] G. T. Fallenstein, V. D. Hulce, and J. W. Melvin. Dynamic mechanical properties of human brain tissue. Journal of Biomechanics, 2(3):217226, 1969.

[26] K. B. Arbogast and S. S. Margulies. Material characterization of the brainstem from oscillatory shear tests. Journal of Biomechanics, 31(9):801-807, 1998.

[27] S. Nicolle, M. Lounis, and R. Willinger. Shear properties of brain tissue over a frequency range relevant for automotive impact situations: new experimental results. Stapp Car Crash Journal, 48:239-258, 2004.

[28] M. Hrapko, J. A. W. van Dommelen, G. W. M. Peters, and J. S. H. M. Wismans. The mechanical behaviour of brain tissue: large strain response and constitutive modelling. Biorheology, 43(5):623-636, 2006. 
[29] S. Chatelin, A. Constantinesco, and R. Willinger. Fifty years of brain tissue mechanical testing: From in vitro to in vivo investigations, 2010.

[30] J. E. Galford and J. H. McElhaney. A viscoelastic study of scalp, brain, and dura. Journal of Biomechanics, 3(2):211-221, 1970.

[31] M. S. Estes and J. H. Mcelhaney. Response of brain tissue to compressive loading. ASME paper no. 70-BHF-13, 1970.

[32] L. Z. Shuck and S. H. Advani. Rheological response of human brain tissue in shear. Journal of Basic Engineering, 94(4):905-911, 1972.

[33] K. Miller and K. Chinzei. Constitutive modelling of brain tissue: experiment and theory. Journal of Biomechanics, 30(11-12):1115-1121, 1997.

[34] K. Miller. Constitutive model of brain tissue suitable for finite element analysis of surgical procedures. Journal of Biomechanics, 32(5):531-537, 1999.

[35] K. Miller, K. Chinzei, G. Orssengo, and P. Bednarz. Mechanical properties of brain tissue in-vivo: Experiment and computer simulation. Journal of Biomechanics, 33(11):1369-1376, 2000.

[36] D. W. A. Brands, P. H. M. Bovendeerd, G. W. M. Peters, J. S. H. M. Wismans, M. H. J. W. Paas, and J. L. M. J. van Bree. Comparison of the dynamic behavior of brain tissue and two model materials. In 43rd Stapp Car Crash Conference Proceedings, 1999.

[37] G.W.M. Peters, J.H. Meulman, and A.A.H.J. Sauren. The applicability of the time/temperature superposition principle to brain tissue. Biorheology, 34(2):127 - 138, 1997.

[38] J. H. McElhaney, J. W. Melvin, V. L. Roberts, and H. D. Portnoy. Dynamic Characteristics of the Tissues of the Head, pages 215-222. Palgrave Macmillan UK, London, 1973.

[39] K. B. Arbogast and S. S. Margulies. Regional differences in mechanical properties of the porcine central nervous system. Proceedings of the 41 st Stapp Car Crash Conference, 1997. 
[40] K. L. Thibault and S. S. Margulies. Material properties of the developing porcine brain. In Proceedings of the international IRCOBI conference on biomechanics of impact, pages 75-85, 1996.

[41] K. L. Thibault and S. S. Margulies. Age-dependent material properties of the porcine cerebrum: Effect on pediatric inertial head injury criteria. Journal of Biomechanics, 31(12):1119-1126, 1998.

[42] L. E. Bilston, Z. Z. Liu, and N. Phan-Thien. Large strain behaviour of brain tissue in shear: Some experimental data and differential constitutive model. Biorheology, 38(4):335-345, 2001.

[43] C. Zhou, T. B. Khalil, and A. I. King. A new model comparing impact responses of the homogeneous and inhomogeneous human brain. Proceedings of the 39th Stapp Car Crash Conference, 1995.

[44] M. T. Prange and S. S. Margulies. Regional, directional, and agedependent properties of the brain undergoing large deformation. Journal of Biomechanical Engineering, 124(2):244-252, 2002.

[45] S. Budday, G. Sommer, J. Haybaeck, P. Steinmann, G. A. Holzapfel, and E. Kuhl. Rheological characterization of human brain tissue. Acta Biomaterialia, 15(60):315-329, 2017.

[46] T. P. Prevost, A. Balakrishnan, S. Suresh, and S. Socrate. Biomechanics of brain tissue. Acta Biomaterialia, 7(1):83-95, 2011.

[47] X. Jin, F. Zhu, H. Mao, M. Shen, and K. H. Yang. A comprehensive experimental study on material properties of human brain tissue. Journal of Biomechanics, 46(16):2795-2801, 2013.

[48] A. E. Forte, S. M. Gentleman, and D. Dini. On the characterization of the heterogeneous mechanical response of human brain tissue. Biomechanics and Modeling in Mechanobiology, 16(3):907-920, 2017.

[49] J. D. Finan, S. N. Sundaresh, B. S. Elkin, G. M. McKhann, and B. Morrison. Regional mechanical properties of human brain tissue for computational models of traumatic brain injury. Acta Biomaterialia, 55:333-339, 2017. 
[50] K. M. Labus and C. M. Puttlitz. An anisotropic hyperelastic constitutive model of brain white matter in biaxial tension and structural-mechanical relationships. Journal of the Mechanical Behavior of Biomedical Materials, 62:195-208, 2016.

[51] P. J. McCracken, A. Manduca, J. Felmlee, and R. L. Ehman. Mechanical transient-based magnetic resonance elastography. Magnetic Resonance in Medicine, 53(3):628-639, 2005.

[52] S. A. Kruse, G. H. Rose, K. J. Glaser, A. Manduca, J. P. Felmlee, C. R. Jack, and R. L. Ehman. Magnetic resonance elastography of the brain. NeuroImage, 39(1):231 - 237, 2008.

[53] I. Sack, K. Jöhrens, J. Würfel, and J. Braun. Structure-sensitive elastography: on the viscoelastic powerlaw behavior of in vivo human tissue in health and disease. Soft Matter, 9:5672-5680, 2013.

[54] M. C. Murphy, J. Huston, and R. L. Ehman. MR elastography of the brain and its application in neurological diseases, 2017.

[55] L. V. Hiscox, C. L. Johnson, M. D. J. McGarry, M. Perrins, A. Littlejohn, E. J. R. van Beek, N. Roberts, and J. M. Starr. High-resolution magnetic resonance elastography reveals differences in subcortical gray matter viscoelasticity between young and healthy older adults. Neurobiology of Aging, 65:158-167, 2018.

[56] M. A. Green, L. E. Bilston, and R. Sinkus. In vivo brain viscoelastic properties measured by magnetic resonance elastography. NMR in Biomedicine, 21(7):755-764, 2008.

[57] A. T. Anderson, E. E. W. Van Houten, M. D. J. McGarry, K. D. Paulsen, J. L. Holtrop, B. P. Sutton, J. G. Georgiadis, and C. L. Johnson. Observation of direction-dependent mechanical properties in the human brain with multi-excitation MR elastography. Journal of the Mechanical Behavior of Biomedical Materials, 59:538-546, 2016.

[58] C. L. Johnson, M. D. J. McGarry, A. A. Gharibans, J. B. Weaver, K. D. Paulsen, H. Wang, W. C. Olivero, B. P. Sutton, and J. G. Georgiadis. Local mechanical properties of white matter structures in the human brain. NeuroImage, 1(79):142-152, 2013. 
[59] J. L. Schmidt, D. J. Tweten, A. A. Badachhape, A. J. Reiter, R. J. Okamoto, J. R. Garbow, and P. V. Bayly. Measurement of anisotropic mechanical properties in porcine brain white matter ex vivo using magnetic resonance elastography. Journal of the Mechanical Behavior of Biomedical Materials, 79:30-37, 2018.

[60] J. Testu, M. D.J. McGarry, F. Dittmann, J. B. Weaver, K. D. Paulsen, I. Sack, and E. E.W. Van Houten. Viscoelastic power law parameters of in vivo human brain estimated by MR elastography. Journal of the Mechanical Behavior of Biomedical Materials, 74:333-341, 2017.

[61] C. L. Johnson, H. Schwarb, M. D.J. McGarry, A. T. Anderson, G. R. Huesmann, B. P. Sutton, and N. J. Cohen. Viscoelasticity of subcortical gray matter structures. Human Brain Mapping, 37(12):4221-4233, 2016.

[62] X. Huang, H. Chafi, K. L. Matthews, O. Carmichael, T. Li, Q. Miao, S. Wang, and G. Jia. Magnetic resonance elastography of the brain: A study of feasibility and reproducibility using an ergonomic pillow-like passive driver. Magnetic Resonance Imaging, 59:68-76, jun 2019.

[63] A. Kolipaka, P. A. Wassenaar, S. Cha, W. M. Marashdeh, X. Mo, P. Kalra, B. Gans, B. Raterman, and E. Bourekas. Magnetic resonance elastography to estimate brain stiffness: Measurement reproducibility and its estimate in pseudotumor cerebri patients. Clinical Imaging, $51: 114-122,2018$.

[64] I. Sack, B. Beierbach, J. Wuerfel, D. Klatt, U. Hamhaber, S. Papazoglou, P. Martus, and J. Braun. The impact of aging and gender on brain viscoelasticity. NeuroImage, 46(3):652-657, 2009.

[65] I. Sack, K. J. Streitberger, D. Krefting, F. Paul, and J. Braun. The influence of physiological aging and atrophy on brain viscoelastic properties in humans. PLoS ONE, 6(9):e23451, 2011.

[66] A.rvin Arani, M. C. Murphy, K. J. Glaser, A. Manduca, D. S. Lake, S. A. Kruse, C. R. Jack, R. L. Ehman, and J. Huston. Measuring the effects of aging and sex on regional brain stiffness with MR elastography in healthy older adults. NeuroImage, 1(111):59-64, 2015.

[67] J. Weickenmeier, M. Kurt, E. Ozkaya, R. de Rooij, T. C. Ovaert, R. L. Ehman, K. Butts Pauly, and E. Kuhl. Brain stiffens post mortem. 
Journal of the Mechanical Behavior of Biomedical Materials, 84:88-98, 2018.

[68] L. A. Mihai, S. Budday, G. A. Holzapfel, E. Kuhl, and A. Goriely. A family of hyperelastic models for human brain tissue. Journal of the Mechanics and Physics of Solids, 106:60-79, 2017.

[69] M R Pamidi and S. H. Advani. Nonlinear Constitutive Relations for Human Brain Tissue. Transactions of the ASME. Journal of Biomechanics, 1978.

[70] K. K. Mendis, R. L. Stalnaker, and S. H. Advani. A constitutive relationship for large deformation finite element modeling of brain tissue. Journal of Biomechanical Engineering, 117(3):279-285, 1995.

[71] S. Budday, G. Sommer, C. Birkl, C. Langkammer, J. Haybaeck, J. Kohnert, M. Bauer, F. Paulsen, P. Steinmann, E. Kuhl, and G. A. Holzapfel. Mechanical characterization of human brain tissue. Acta Biomaterialia, 48:319-340, 2017.

[72] D. W. A. Brands, G. W. M. Peters, and P. H. M. Bovendeerd. Design and numerical implementation of a 3-D non-linear viscoelastic constitutive model for brain tissue during impact. Journal of Biomechanics, 2004.

[73] K. Haldar and C. Pal. Rate dependent anisotropic constitutive modeling of brain tissue undergoing large deformation. Journal of the Mechanical Behavior of Biomedical Materials, 81:178-194, 2018.

[74] A. Tamura, S. Hayashi, I. Watanabe, K. Nagayama, and T. Matsumoto. Mechanical characterization of brain tissue in high-rate compression. Journal of Biomechanical Science and Engineering, 2(3):115-126, 2007.

[75] A. Mehrabian and Y. Abousleiman. General solutions to poroviscoelastic model of hydrocephalic human brain tissue. Journal of Theoretical Biology, 291:105-118, 2011.

[76] L. Sakka and J. Chazal. Les méninges vues par l'anatomiste. Morphologie, 89(284):35-42, mar 2005.

[77] D. B. MacManus, B. Pierrat, J. G. Murphy, and M. D. Gilchrist. Protection of cortex by overlying meninges tissue during dynamic indentation of the adolescent brain. Acta Biomaterialia, 15(57):384-394, 2017. 
[78] J. A. Siegenthaler and S. J. Pleasure. We have got you 'covered': How the meninges control brain development, 2011.

[79] R. Rua and D. B. McGavern. Advances in Meningeal Immunity. Trends in Molecular Medicine, 24(6):542-559, jun 2018.

[80] N. Patel and O. Kirmi. Anatomy and Imaging of the Normal Meninges. Seminars in Ultrasound, CT and MRI, 2009.

[81] R. van Noort, M. M. Black, T. R. P. Martin, and S. Meanley. A study of the uniaxial mechanical properties of human dura mater preserved in glycerol. Biomaterials, 2(1):41-45, jan 1981.

[82] M. C. Jimenez Hamann, M. S. Sacks, and T. I. Malinin. Quantification of the collagen fibre architecture of human cranial dura mater. Journal of Anatomy, 192(1):99-106, 1998. cited By 23.

[83] D. De Kegel, J. Vastmans, H. Fehervary, B. Depreitere, J. Vander Sloten, and N. Famaey. Biomechanical characterization of human dura mater. Journal of the Mechanical Behavior of Biomedical Materials, 79:122-134, 2018.

[84] R. O. Weller. Microscopic morphology and histology of the human meninges. Morphologie, 89(284):22-34, mar 2005.

[85] A. Granger, S. Kim, T. Kollias, and T. Rajnauth. Ossification of the falx cerebri: A review. Translational Research in Anatomy, 7:20-22, jun 2017.

[86] D. R. Walsh, A. M. Ross, S. Malijauskaite, B. D. Flanagan, D. T. Newport, K. D. McGourty, and John J. E. Mulvihill. Regional mechanical and biochemical properties of the porcine cortical meninges. Acta Biomaterialia, 80:237-246, oct 2018.

[87] J. W. Melvin, J. H. McElhaney, and V. L. Roberts. Development of a Mechanical Model of the Human Head - Determination of Tissue Properties and Synthetic Substitute Materials. In 14th Stapp Car Crash Conference. SAE International, feb 1970.

[88] E. Raposio and R. E. A. Nordström. Tension and flap advancement in the human scalp. Annals of Plastic Surgery, 39(1):20-23, 1997. 
[89] L. Falland-Cheung, M. Scholze, P. F. Lozano, B. Ondruschka, D. C. Tong, P. A. Brunton, J. N. Waddell, and N. Hammer. Mechanical properties of the human scalp in tension. Journal of the Mechanical Behavior of Biomedical Materials, 84:188-197, aug 2018.

[90] N. Pittar, T. Winter, L. Falland-Cheung, D. Tong, and J.N. Waddell. Scalp simulation â A novel approach to site-specific biomechanical modeling of the skin. Journal of the Mechanical Behavior of Biomedical Materials, 77:308-313, jan 2018.

[91] R. Åstrand and B. Romner. Management of severe traumatic brain injury: evidence, tricks, and pitfalls, pages 11-16. Springer Berlin Heidelberg, Berlin, Heidelberg, 2012.

[92] J. W. Bales and R. H. Bonow. Closed Head Injury. Principles of Neurological Surgery, 79:366-389, jan 2018.

[93] C. Werner and K. Engelhard. Pathophysiology of traumatic brain injury. BJA: British Journal of Anaesthesia, 99(1):4-9, 2007.

[94] H. J. Carson. Brain trauma in head injuries presenting with and without concurrent skull fractures. Journal of Forensic and Legal Medicine, 16(3):115-120, 2009.

[95] J. D. Miller and R. Nader. Acute subdural hematoma from bridging vein rupture: a potential mechanism for growth. Journal of Neurosurgery, 120(6):1378-1384, 2014.

[96] P. Johns. Chapter 9 - Head injury. Clinical Neuroscience, pages 105-114, jan 2014.

[97] V. E. Johnson, W. Stewart, and D. H. Smith. Axonal pathology in traumatic brain injury, 2013.

[98] D. H. Smith, D. F. Meaney, and W. H. Shull. Diffuse axonal injury in head trauma, 2003.

[99] G. Teasdale and B. Jennett. Assessment of Coma and Impaired Conciousness: A Practical Scale. The Lancet, 304(7872):81-84, jul 1974. 
[100] E. S. Gurdjian, V. L. Roberts, and L. M. Thomas. Tolerance curves of acceleration and intracranial pressure and protective index in experimental head injury. Journal of Trauma - Injury, Infection and Critical Care, 1966.

[101] E. S. Gurdjian, J. E. Webster, and H. R. Lissner. Observations on the mechanism of brain concussion, contusion, and laceration. Surgery, gynecology $\&$ obstetrics, 1955.

[102] E. S. Gurdjian, H. R. Lissner, F. G. Evans, L. M. Patrick, and W. G. Hardy. Intracranial pressure and acceleration accompanying head impacts in human cadavers. Surg Gynecol Obstet, 113:185-190, 1961.

[103] C. W. Gadd. Use of a weighted-impulse criterion for estimating injury hazard. In 10th Stapp Car Crash Conference (1966). SAE International, feb 1966 .

[104] J. Versace. A review of the severity index. In Proceedings of the 15th Stapp Car Crash Conference, pages 771-796, 1971.

[105] National Highway Traffic Safety Administration. Occupant crash protection - Head injury criterion. Report no. FMVSS 208 for the US Department of Transportation, 1972.

[106] J. Hutchinson, M. J. Kaiser, and H. M. Lankarani. The Head Injury Criterion (HIC) functional. Applied Mathematics and Computation, 96(1):1-16, 1998.

[107] R. L. Stalnaker, J. L. Fogle, and J. H. McElhaney. Driving point impedance characteristics of the head. Journal of Biomechanics, 4(2):127 - 139, 1971.

[108] C. Got, G. Walfisch, C. Tarriere, A. Fayon, and A. Patel. Results of experimental head impacts on cadavers: the various data obtained and their relations to some measured physical parameters. Stapp Car Crash Conference. Twenty-Second. Proceedings. Warrendale, Society of Automotive Engineers, pages 55-99, 1978.

[109] C. Tarriere, G. Walfisch, A. Fayon, C. Got, F. Guillon, A. Patel, and J. Bureau. Acceleration, jerk and neck flexion angle: Their respective 
influences on the occurrence of brain injury. ISO/TC 22/SC 12/GT-6 (USA-13) Doc No. 118., 1983.

[110] H. Prasad, P.and J. Mertz. The position oft he united states delegation to the iso working group 6 on the use of hic in automotive environment. SAE Government Industry Meeting and ExpositionSAE Government Industry Meeting and Exposition, 011985.

[111] H. J. Mertz and D. A. Weber. Interpretations of the impact responses of a 3-year-old child dummy relative to child injury potential. Proceedings of the 9th International Technical Conference on Experimental Safety Vehicles (ESV), 1982.

[112] E. Hertz. A note on the head injury criterion (HIC) as a predictor of the risk of skull fracture. In Proceedings of the Association for the Advancement of Automotive Medicine Annual Conference, 1993.

[113] J. Ruan and P. Prasad. The effects of skull thickness variations on human head dynamic impact responses. Stapp car crash journal, 45:395-414, 2001.

[114] H. J. Mertz, P. Prasad, and G. S. Nusholtz. Head injury risk assessment for forehead impacts. SAE Transactions, 105(6):22-46, 1996.

[115] M. Vander Vorst, J. Stuhmiller, K. Ho, N. Yoganandan, and F. Pintar. Statistically and biomechanically based criterion for impact-induced skull fracture. Annual proceedings / Association for the Advancement of Automotive Medicine. Association for the Advancement of Automotive Medicine, 2003.

[116] M. Vander Vorst, P. Chan, J. Zhang, N. Yoganandan, and F. Pintar. A new biomechanically-based criterion for lateral skull fracture. Annual proceedings / Association for the Advancement of Automotive Medicine. Association for the Advancement of Automotive Medicine, 2004.

[117] J. A. Newman. A generalized model for brain injury threshold (GAMBIT). In Proceedings of the 1986 International IRCOBI Conference on the Biomechanics of Impact, pages 121-131, 1986.

[118] J. A. Newman, N. Shewchenko, and E. Welbourne. A new biomechanical assessment of mild traumatic brain injury. Stapp Car Crash Journal, 44(215-247), 2000. 
[119] J. A. Newman, C. Barr, M. Beusenberg, E. Fournier, N. Shewchenko, E. Welbourne, and C. Withnall. A new biomechanical assessment of mild traumatic brain injury. Part 2: results and conclusions. In Proceedings of the 2000 International IRCOBI Conference on the Biomechanics of Impact, pages 223-233, 2000.

[120] A. H.S. Holbourn. Mechanics of head injuries. The Lancet, 1943.

[121] S. J. Strich. Diffuse degeneration of the cerebral white matter in severe dementia following head injury. Journal of neurology, neurosurgery, and psychiatry, 1956.

[122] S. J. Strich. Shearing of nerve fibres as a cause of brain damage due to head injury. A Pathological Study of Twenty Cases. The Lancet, 1961.

[123] S. J. Peerless and N. B. Rewcastle. Shear Injuries of the Brain. Canadian Medical Association journal, 1967.

[124] A. K. Ommaya and T. A. Gennarelli. Cerebral concussion and traumatic unconsciousness: Correlation of experimental and clinical observat. Brain : a journal of neurology, 97(4):633-654, 1975.

[125] T. A. Gennarelli, L. E. Thibault, J. H. Adams, D. I. Graham, C. J. Thompson, and R. P. Marcincin. Diffuse axonal injury and traumatic coma in the primate. Annals of Neurology, 12(6):564-574, 1982.

[126] S. S. Margulies and L. E. Thibault. A proposed tolerance criterion for diffuse axonal injury in man. Journal of Biomechanics, 25(8):917-923, 1992.

[127] F A Bandak and R H Eppinger. A three-dimensional finite element analysis of the human brain under combined rotational and translational accelerations. Stapp Car Crash Conference, STAPP 1994, 1994.

[128] E. G. Takhounts, R. H. Eppinger, J. Q. Campbell, and R. E. Tannous. On the development of the SIMon finite element head model. Stapp Car Crash Journal, 2003.

[129] L. Zhang, K. H. Yang, and A. I. King. A proposed injury threshold for mild traumatic brain injury. Journal of Biomechanical Engineering, 126(2):226-236, 2004. 
[130] L Zhang, K H Yang, and A I King. Comparison of brain responses between frontal and lateral impacts by finite element modeling. Journal of Neurotrauma, 18(1):21-30, 2001.

[131] D. I. Shreiber, A. C. Bain, and D. F. Meaney. In Vivo Threshlolds for Mechanical Injury to the Blood-Brain Barrier. SAE technical paper, 1997.

[132] A. C. Bain and D. F. Meaney. Tissue-Level Thresholds for Axonal Damage in an Experimental Model of Central Nervous System White Matter Injury. Journal of Biomechanical Engineering, 2000.

[133] B. Morrison, H. L. Cater, C. C.-B. Wang, F. C. Thomas, C. T. Hung, G. A. Ateshian, and L. E. Sundstrom. A Tissue Level Tolerance Criterion for Living Brain Developed with an In Vitro Model of Traumatic Mechanical Loading. Stapp Car Crash Journal, 2003.

[134] D. Sahoo, C. Deck, and R. Willinger. Brain injury tolerance limit based on computation of axonal strain. Accident Analysis \& Prevention, 92:5370, jul 2016.

[135] A. G. Monea, G. Van der Perre, K. Baeck, H. Delye, P. Verschueren, E. Forausebergher, C. Van Lierde, I. Verpoest, J. Vander Sloten, J. Goffin, and B. Depreitere. The relation between mechanical impact parameters and most frequent bicycle related head injuries. Journal of the Mechanical Behavior of Biomedical Materials, 33(1):3-15, 2014.

[136] H. Delye, J. Goffin, P. Verschueren, J. Vander Sloten, G. Van der Perre, H. Alaerts, I. Verpoest, and D. Berckmans. Biomechanical properties of the superior sagittal sinus-bridging vein complex. Stapp Car Crash Journal, 50:625-636, 2006.

[137] B. Depreitere, C. Van Lierde, J. Vander Sloten, R. Van Audekercke, G. Van Der Perre, C. Plets, and J. Goffin. Mechanics of acute subdural hematomas resulting from bridging vein rupture. Journal of Neurosurgery, 2006.

[138] K. L. Monson, W. Goldsmith, N. M. Barbaro, and G. T. Manley. Axial Mechanical Properties of Fresh Human Cerebral Blood Vessels. Journal of Biomechanical Engineering, 2003. 
[139] K. L. Monson, W. Goldsmith, N. M. Barbaro, and G. T. Manley. Significance of source and size in the mechanical response of human cerebral blood vessels. Journal of Biomechanics, 2005.

[140] A. G. Monea, K. Baeck, E. Verbeken, I. Verpoest, J. Vander Sloten, J. Goffin, and B. Depreitere. The biomechanical behaviour of the bridging vein-superior sagittal sinus complex with implications for the mechanopathology of acute subdural haematoma. Journal of the Mechanical Behavior of Biomedical Materials, 32:155-165, 2014.

[141] C. C. Ward, M. Chan, and A. M. Nahum. Intracranial Pressure - A Brain Injury Criterion. SAE Technical Paper 801304, 1980.

[142] A. M. S. Hamouda, R. M. Sohaimi, A. M. A. Zaidi, and S. Abdullah. Materials and design issues for military helmets. Woodhead Publishing Limited, 2012.

[143] T. El Sayed, A. Mota, F. Fraternali, and M. Ortiz. Biomechanics of traumatic brain injury. Computer Methods in Applied Mechanics and Engineering, 197(51-52):4692-4701, 2008.

[144] A. G. Gross. A new theory on the dynamics of brain concussion and brain injury, 1958.

[145] P. Lubock and W. Goldsmith. Experimental cavitation studies in a model head-neck system. Journal of Biomechanics, 13(12):1041-1052, 1980.

[146] M. B. Panzer, B. S. Myers, B. P. Capehart, and C. R. Bass. Development of a finite element model for blast brain injury and the effects of CSF cavitation. Annals of Biomedical Engineering, 40(7):1530-1544, 2012.

[147] D. Jenson and V. U. Unnikrishnan. Energy dissipation of nanocomposite based helmets for blast-induced traumatic brain injury mitigation. Composite Structures, 121:211-216, 2015.

[148] D. Sahoo, C. Robbe, C. Deck, F. Meyer, A. Papy, and R. Willinger. Head injury assessment of non-lethal projectile impacts: A combined experimental/computational method. Injury, 2016. 
[149] A. K. Ommaya, R. L. Grubb, and R. A. Naumann. Coup and contrecoup injury: observations on the mechanics of visible brain injuries in the rhesus monkey. Journal of Neurosurgery, 35(5):503-516, 1971.

[150] N. Baddour. Hamilton's Principle for the Derivation of Equations of Motion, pages 155-182. 112007.

[151] S. R. Wu and L. Gu. Introduction to the Explicit Finite Element Method for Nonlinear Transient Dynamics. 2012.

[152] J. S. Sun, K. H. Lee, and H. P. Lee. Comparison of implicit and explicit finite element methods for dynamic problems. Journal of Materials Processing Technology, 105(1-2):110-118, sep 2000.

[153] T. Belytschko, T. J. R. Hughes, and P. Burgers. Computational Methods for Transient Analysis. Computational Methods in Mechanics (Volume 1), 1983.

[154] L. Maheo, V. Grolleau, and G. Rio. Numerical damping of spurious oscillations: A comparison between the bulk viscosity method and the explicit dissipative Tchamwa-Wielgosz scheme. Computational Mechanics, 51(1):109-128, 2013.

[155] Dassault Systèmes. Abaqus 6.12 User's Manual. 2012.

[156] S. R. Wu. Lumped mass matrix in explicit finite element method for transient dynamics of elasticity. Computer Methods in Applied Mechanics and Engineering, 195(44-47):5983-5994, sep 2006.

[157] T. Belytschko and L. P. Bindeman. Assumed strain stabilization of the eight node hexahedral element. Computer Methods in Applied Mechanics and Engineering, 1993.

[158] D. P. Flanagan and T. Belytschko. A uniform strain hexahedron and quadrilateral with orthogonal hourglass control. International Journal for Numerical Methods in Engineering, 17(5):679-706, 1981.

[159] R. L. Taylor and P. Papadopoulos. On a finite element method for dynamic contact/impact problems. International Journal for Numerical Methods in Engineering, 1993. 
[160] I. Huněk. On a penalty formulation for contact-impact problems. Computers and Structures, 1993.

[161] N. J. Carpenter, R. L. Taylor, and M. G. Katona. Lagrange constraints for transient finite element surface contact. International Journal for Numerical Methods in Engineering, 1991.

[162] S. R. Wu. A variational principle for dynamic contact with large deformation. Computer Methods in Applied Mechanics and Engineering, 198(21-26):2009-2015, may 2009.

[163] M. Freitas, A. Silva, and L. Reis. Numerical evaluation of failure mechanisms on composite specimens subjected to impact loading. Composites Part B: Engineering, 31(3):199 - 207, 2000.

[164] C. Bouvet, S. Rivallant, and J. J. Barrau. Low velocity impact modeling in composite laminates capturing permanent indentation. Composites Science and Technology, 72(16):1977-1988, nov 2012.

[165] H. Tuo, Z. Lu, X. Ma, J. Xing, and C. Zhang. Damage and failure mechanism of thin composite laminates under low-velocity impact and compression-after-impact loading conditions. Composites Part B: Engineering, 163:642-654, apr 2019.

[166] F. K. Chang and K. Y. Chang. A progressive damage model for laminated composites containing stress concentrations. Journal of Composite Materials, 21(9):834-855, 1987.

[167] N. K. Naik, Y. Chandra Sekher, and S. Meduri. Damage in wovenfabric composites subjected to low-velocity impact. Composites Science and Technology, 60(5):731-744, apr 2000.

[168] I. K. Giannopoulos, E. E. Theotokoglou, and X. Zhang. Impact damage and CAI strength of a woven CFRP material with fire retardant properties. Composites Part B: Engineering, 91:8-17, apr 2016.

[169] M. Grasso, Y. Xu, A. Ramji, G. Zhou, A. Chrysanthou, G. Haritos, and Y. Chen. Low-velocity impact behaviour of woven laminate plates with fire retardant resin. Composites Part B: Engineering, 171:1-8, aug 2019. 
[170] H. Ullah, A. R. Harland, and V. V. Silberschmidt. Damage in woven cfrp laminates under impact loading. EPJ Web of Conferences, 26:04004, 2012.

[171] B. I. S. Murat and A. A. A. Rahman. Study of impact damage behavior in woven carbon fiber plates. Procedia Engineering, 170:47 - 54, 2017. Engineering Physics International Conference 2016 â EPIC 2016.

[172] B. Zhang, Z. Yang, X. Sun, and Z. Tang. A virtual experimental approach to estimate composite mechanical properties: Modeling with an explicit finite element method. Computational Materials Science, 49(3):645-651, sep 2010.

[173] P. A. A. E. Mendes and M. V. Donadon. Numerical prediction of compression after impact behavior of woven composite laminates. Composite Structures, 113:476-491, jul 2014.

[174] L. A. Coles, A. Roy, and V. V. Silberschmidt. Ice vs. steel: Ballistic impact of woven carbon/epoxy composites. Part II â Numerical modelling. Engineering Fracture Mechanics, 24(79):30-37, jan 2019.

[175] D. Delfosse and A. Poursartip. Energy-based approach to impact damage in CFRP laminates. Composites Part A: Applied Science and Manufacturing, 28(7):647-655, jan 1997.

[176] W. H. Peters and W. F. Ranson. Digital imaging techniques in experimental stress analysis. Optical Engineering, 21(3):427 - 431 - 5, 1982.

[177] M. A. Sutton, W. J. Wolters, W. H. Peters, W. F. Ranson, and S. R. McNeill. Determination of displacements using an improved digital correlation method. Image and Vision Computing, 1(3):133-139, 1983.

[178] H. Schreier, J. J. Orteu, and M. A. Sutton. Image correlation for shape, motion and deformation measurements: Basic concepts, theory and applications. 2009.

[179] B. D. Lucas and T. Kanade. An iterative image registration technique with an application to stereo vision. In Proceedings of the 1981 DARPA Image Understanding Workshop, pages 121-130, 1981. 
[180] C. S. Lopes, P. P. Camanho, Z. Gürdal, P. Maimí, and E. V. González. Low-velocity impact damage on dispersed stacking sequence laminates. Part II: Numerical simulations. Composites Science and Technology, 69(7-8):937-947, jun 2009.

[181] R. Higuchi, T. Okabe, A. Yoshimura, and T. E. Tay. Progressive failure under high-velocity impact on composite laminates: Experiment and phenomenological mesomodeling. Engineering Fracture Mechanics, 178:346-361, jun 2017.

[182] C. Zhang, E. A. Duodu, and J. Gu. Finite element modeling of damage development in cross-ply composite laminates subjected to low velocity impact. Composite Structures, 173:219-227, aug 2017.

[183] Y. Wang, S. Zhu, J. Qi, and J. Xiao. Development of a finite element model for progressive damage analysis of composite laminates subjected to low velocity impact. Polymers and Polymer Composites, 22:73-78, 01 2014.

[184] Dassault Systèmes. Section 1.2.19 VUSDFLD, Abaqus User Subroutines Reference Manual, 2012.

[185] J. Körbelin, M. Derra, and B. Fiedler. Influence of temperature and impact energy on low velocity impact damage severity in CFRP. Composites Part A: Applied Science and Manufacturing, 115:76-87, dec 2018.

[186] L. Voo, S. Kumaresan, F. A. Pintar, N. Yoganandan, and A. Sances. Finite-element models of the human head, 1996.

[187] J. S. Raul, C. Deck, R. Willinger, and B. Ludes. Finite-element models of the human head and their applications in forensic practice, 2008.

[188] F. Tarlochan and H. Samaka. Finite Element (FE) human head models: literature review. International Journal of Scientific \&3 Technology Research, 2(7):17-31, 2013.

[189] K. M. Tse, S. P. Lim, V. Beng, C. Tan, and H. P. Lee. A review of head injury and finite element head models. American Journal of Engineering , Technology and Society American Journal of Engineering Technology and Society, 2014. 
[190] C. Conrad Ward and R. B. Thompson. The development of a detailed finite element brain model. In 19th Stapp Car Crash Conference (1975). SAE International, feb 1975.

[191] A. M. Nahum, R. Smith, and C. C. Ward. Intracranial Pressure Dynamics During Head Impact. feb 1977.

[192] J. S. Ruan, T. B. Khalil, and A. I. King. Finite element modeling of direct head impact. SAE Technical Papers, 1993.

[193] J. S. Ruan, T. Khalil, and A. I. King. Dynamic response of the human head to impact by three-dimensional finite element analysis. Journal of Biomechanical Engineering, 116(1):44-50, 1994.

[194] L. Zhang, K. Yang, R. Dwarampudi, K. Omori, T. Li, K. Chang, W. N. Hardy, T. B. Khalil, and A. King. Recent advances in brain injury research: a new human head model development and validation. Stapp car crash journal, 45:369-94, 122001.

[195] H.-S. Kang, R. Willinger, B. M. Diaw, and B. Chinn. Validation of a 3D Anatomic Human Head Model and Replication of Head Impact in Motorcycle Accident by Finite Element Modeling. Stapp Car Crash Conference, 1997.

[196] R. Willinger, H. S. Kang, and B. Diaw. Three-dimensional human head finite-element model validation against two experimental impacts. Annals of Biomedical Engineering, 27(3):403-410, 1999.

[197] R. Willinger and D. Baumgartner. Human head tolerance limits to specific injury mechanisms. International Journal of Crashworthiness, 8(6):605-617, 2003.

[198] C. Deck and R. Willinger. Head injury prediction tool for protective systems optimization. In 7th European LS-DYNA Conference, 2009.

[199] S. Kleiven and W. N. Hardy. Correlation of an FE Model of the human head with local brain motion-consequences for injury prediction. Stapp car crash journal, 46:123-144, 2002.

[200] C. Giordano, S. Zappalà, and S. Kleiven. Anisotropic finite element models for brain injury prediction: the sensitivity of axonal strain to 
white matter tract inter-subject variability. Biomechanics and Modeling in Mechanobiology, 16(4):1269-1293, 2017.

[201] T. J. Horgan and M. D. Gilchrist. The creation of three-dimensional finite element models for simulating head impact biomechanics. International Journal of Crashworthiness, 8:353-366, 2003.

[202] A. Post, T. Blaine Hoshizaki, M. D. Gilchrist, and M. D. Cusimano. Peak linear and rotational acceleration magnitude and duration effects on maximum principal strain in the corpus callosum for sport impacts. Journal of Biomechanics, 61:183-192, 2017.

[203] K. M. Tse, L. B. Tan, S. J. Lee, S. P. Lim, and H. P. Lee. Development and validation of two subject-specific finite element models of human head against three cadaveric experiments. International Journal for Numerical Methods in Biomedical Engineering, 30(3):397-415, 2014.

[204] K. M. Tse, L. B. Tan, B. Yang, V. B. C. Tan, and H. P. Lee. Effect of helmet liner systems and impact directions on severity of head injuries sustained in ballistic impacts: a finite element (FE) study. Medical and Biological Engineering and Computing, 55(4):641-662, 2017.

[205] NASA. NASA-STD-3000 Man-Systems Integration Standards Volume I. $1995,1995$.

[206] Simpleware Ltd. ScanIP Reference Guide Version 4.2, 2010.

[207] H. Kasban, M. A. M. El-Bendary, and D. H. Salama. A Comparative Study of Medical Imaging Techniques. International Journal of Information Science and Intelligent System, 2015.

[208] S. Boruah, G. R. Paskoff, B. S. Shender, D. L. Subit, R. S. Salzar, and J. R. Crandall. Variation of bone layer thicknesses and trabecular volume fraction in the adult male human calvarium. Bone, 77:120-134, 2015.

[209] A. Voie, M. Dirnbacher, D. Fisher, and T. Hölscher. Parametric mapping and quantitative analysis of the human calvarium. Computerized Medical Imaging and Graphics, 38(8):675-682, dec 2014. 
[210] Q. Grimal and P. Laugier. Quantitative Ultrasound Assessment of Cortical Bone Properties Beyond Bone Mineral Density. IRBM, 40(1):16-24, feb 2019.

[211] S. Kleiven. Influence of Impact Direction on the Human Head in Prediction of Subdural Hematoma. Journal of Neurotrauma, 2003.

[212] S. W. Sloan and M. F. Randolph. Numerical prediction of collapse loads using finite element methods. International Journal for Numerical and Analytical Methods in Geomechanics, 1982.

[213] G. N. Wells, L. J. Sluys, and R. de Borst. A p-adaptive scheme for overcoming volumetric locking during plastic flow. Computer Methods in Applied Mechanics and Engineering, 191(29-30):3153-3164, may 2002.

[214] M. Chiumenti, Q. Valverde, C. Agelet de Saracibar, and M. Cervera. A stabilized formulation for incompressible plasticity using linear triangles and tetrahedra. International Journal of Plasticity, 20(8-9):1487-1504, aug 2004.

[215] P. Y. Rohan, C. Lobos, M. Ali Nazari, P. Perrier, and Y. Payan. Finite element modelling of nearly incompressible materials and volumetric locking: a case study. Computer Methods in Biomechanics and Biomedical Engineering, 2014.

[216] W. M. Coombs, T. J. Charlton, M. Cortis, and C. E. Augarde. Overcoming volumetric locking in material point methods. Computer Methods in Applied Mechanics and Engineering, 333:1-21, may 2018.

[217] M. D. Gilchrist and D. O'Donoghue. Simulation of the development of frontal head impact injury. Computational Mechanics, 26(2):229-235, 2000.

[218] S. Kleiven. Evaluation of head injury criteria using a finite element model validated against experiments on localized brain motion, intracerebral acceleration, and intracranial pressure. Int J Crashworthines, 11(1):65$79,2006$.

[219] K. M. Tse. Development of a realistic finite element model of human head and its applications to head injuries. 2013. 
[220] J. S. Raul, D. Baumgartner, R. Willinger, and B. Ludes. Finite element modelling of human head injuries caused by a fall. International Journal of Legal Medicine, 120(4):212-218, 2006.

[221] F. Silver. Biomaterials, medical devices and tissue engineering: An integrated approach. 1994.

[222] J.-C. Sarron, M. Dannawi, A. Faure, J.-P. Caillou, J. Da Cunha, and R. Robert. Dynamic effects of a $9 \mathrm{~mm}$ missile on cadaveric skull protected by aramid, polyethylene or aluminum plate: an experimental study. The Journal of trauma, 57(2):236-242, 2004.

[223] S.G. Kulkarni, X.-L. Gao, S.E. Horner, J.Q. Zheng, and N.V. David. Ballistic helmets â their design, materials, and performance against traumatic brain injury. Composite Structures, 101:313 - 331, 2013.

[224] J. Van Hoof, D. S. Cronin, M. J. Worswick, K. V. Williams, and D. Nandlall. Numerical Head and Composite Helmet Models To Predict Blunt Trauma. 19th International Symposium of Ballistics, 2001.

[225] D. M. Hisley, J. C. Gurganus, and A. W. Drysdale. Experimental methodology using digital image correlation to assess ballistic helmet blunt trauma. Journal of Applied Mechanics-Transactions of the ASME, 78(5):051022, 2011.

[226] K. A. Rafaels, H. C. Cutcliffe, R. S. Salzar, M. Davis, B. Boggess, B. Bush, R. Harris, M. S. Rountree, E. Sanderson, S. Campman, S. Koch, and C. R. Bass. Injuries of the head from backface deformation of ballistic protective helmets under ballistic impact. Journal of Forensic Sciences, 60(1):219-225, 2015.

[227] C. J. Freitas, J. T. Mathis, N. Scott, R. P. Bigger, and J. MacKiewicz. Dynamic response due to behind helmet blunt trauma measured with a human head surrogate. International Journal of Medical Sciences, 11(5):409-425, 2014.

[228] M. Aare and S. Kleiven. Evaluation of head response to ballistic helmet impacts using the finite element method. International Journal of Impact Engineering, 34(3):596-608, mar 2007. 
[229] S. Kleiven and H. Von Holst. Consequences of reduced brain volume following impact in prediction of subdural hematoma evaluated with numerical techniques. Traffic Injury Prevention, 3(4):303-310, 2002.

[230] Y. Q. Li, X. G. Li, and X.-L. Gao. Modeling of Advanced Combat Helmet under ballistic impact. Journal of Applied Mechanics, 82(11):111004, 2015.

[231] M. Rodríguez-Millán, T. Ito, J. A. Loya, A. Olmedo, and M. H. Miguélez. Development of numerical model for ballistic resistance evaluation of combat helmet and experimental validation. Materials $\&$ Design, 110:391-403, nov 2016.

[232] NIJ Standard 0106.01 for Ballistic Helmets, US Department of Justice, 1981.

[233] X. G. Li, X.-L. Gao, and S. Kleiven. Behind helmet blunt trauma induced by ballistic impact: A computational model. International Journal of Impact Engineering, 91:56-67, may 2016.

[234] S. Kleiven. Predictors for traumatic brain injuries evaluated through accident reconstructions. Stapp Car Crash Journal, 51:81-114, 2007.

[235] R. Eppinger, E. Sun, F. Bandak, M. Haffner, N. Khaewpong, M. Maltese, S. Kuppa, T. Nguyen, E. Takhounts, R. Tannous, A. Zhang, and R. Saul. Development of improved injury criteria for the assessment of advanced automotive restraint systemsâII. National Highway Traffic Safety Administration, 1999.

[236] B. C. M. Macpherson, P. Macpherson, and B. Jennett. CT evidence of intracranial contusion and haematoma in relation to the presence, site and type of skull fracture. Clinical Radiology, 42(5):321-326, 1990.

[237] T. J. Gibson and K. Thai. Helmet protection against basilar skull fracture. Human Impact Engineering. 2007.

[238] M. Samii and M. Tatagiba. Skull base trauma: diagnosis and management. Neurological research, 24(2):147-156, 2002.

[239] T. G. Zhang, S. S. Satapathy, L. R. Vargas-Gonzalez, and S. M. Walsh. Ballistic impact response of Ultra-High-Molecular-Weight Polyethylene (UHMWPE). Composite Structures, 2015. 
[240] H. Van Der Werff and U. Heisserer. High-performance ballistic fibers: Ultra-high molecular weight polyethylene (UHMWPE). Advanced Fibrous Composite Materials for Ballistic Protection, pages 71-107, 2016.

[241] K. Karthikeyan, S. Kazemahvazi, and B. P. Russell. Optimal fibre architecture of soft-matrix ballistic laminates. International Journal of Impact Engineering, 88:227-237, feb 2016.

[242] S. Austin, A. D. Brown, J. P. Escobedo, H. Wang, H. Kleine, and P. J. Hazell. The high-velocity impact of Dyneema® and Spectra@ laminates: implementation of a simple thermal softening model. Procedia Engineering, 204:51-58, jan 2017.

[243] M. K. Hazzard, S. Hallett, P. T. Curtis, L. Iannucci, and R. S. Trask. Effect of fibre orientation on the low velocity impact response of thin Dyneema@ composite laminates. International Journal of Impact Engineering, 100:35-45, feb 2017.

[244] J. G. H. Bouwmeester, R. Marissen, and O. K. Bergsma. Carbon/Dyneema ${ }^{\circledR}$ intralaminar hybrids: new strategy to increase impact resistance or decrease mass of carbon fiber composites. 26th International congress of the aeronautical sciences, 2008.

[245] B.Z. Jang, L.C. Chen, C.Z. Wang, H.T. Lin, and R.H. Zee. Impact resistance and energy absorption mechanisms in hybrid composites. Composites Science and Technology, 34(4):305 - 335, 1989.

[246] M. Grujicic, B. Pandurangan, K. L. Koudela, and B. A. Cheeseman. A computational analysis of the ballistic performance of light-weight hybrid composite armors. Applied Surface Science, 2006.

[247] R.J. Muhi, F. Najim, and M.F.S.F. de Moura. The effect of hybridization on the gfrp behavior under high velocity impact. Composites Part B: Engineering, 40(8):798 - 803, 2009.

[248] P. J. Hazell and G. Appleby-Thomas. A study on the energy dissipation of several different CFRP-based targets completely penetrated by a high velocity projectile. Composite Structures, 2009.

[249] A. K. Bandaru, L. Vetiyatil, and S. Ahmad. The effect of hybridization on the ballistic impact behavior of hybrid composite armors. Composites Part B: Engineering, 76:300-319, 2015. 
[250] T. Lässig, L. Nguyen, M. May, W. Riedel, U. Heisserer, H. Van Der Werff, and S. Hiermaier. A non-linear orthotropic hydrocode model for ultra-high molecular weight polyethylene in impact simulations. International Journal of Impact Engineering, 2015.

[251] J. L. Abot, A. Yasmin, A. J. Jacobsen, and I. M. Daniel. In-plane mechanical, thermal and viscoelastic properties of a satin fabric carbon/epoxy composite. Composites Science and Technology, 64(2):263268 , feb 2004.

[252] Y. Yang and X. Chen. Investigation of failure modes and influence on ballistic performance of Ultra-High Molecular Weight Polyethylene (UHMWPE) uni-directional laminate for hybrid design. Composite Structures, 174:233-243, 2017.

[253] L. H. Nguyen, T. R. Lässig, S. Ryan, W. Riedel, A. P. Mouritz, and A. C. Orifici. Numerical modelling of ultra-high molecular weight polyethylene composite under impact loading. In Procedia Engineering, 2015.

[254] M. K. Hazzard, R. S. Trask, U. Heisserer, M. Van Der Kamp, and S. R. Hallett. Finite element modelling of Dyneema ${ }^{\circledR}$ composites: From quasistatic rates to ballistic impact. Composites Part A: Applied Science and Manufacturing, 115:31-45, dec 2018.

[255] M. Matsumae, R. Kikinis, I. A. Mórocz, A. V. Lorenzo, T. Sándor, M. S. Albert, P. McL. Black, and F. A. Jolesz. Age-related changes in intracranial compartment volumes in normal adults assessed by magnetic resonance imaging. Journal of Neurosurgery, 84(6):982-991, 1996.

[256] W. N. Hardy, C. D. Foster, M. J. Mason, K. H. Yang, and King A. I. Investigation of head injury mechanisms using neutral density technology and high-speed biplanar x-ray. Stapp Car Crash Journal, 45:337-368, 2001.

[257] W. N. Hardy, M. J. Mason, C. D. Foster, C. S. Shah, J. M. Kopacz, K. H. Yang, A. I. King, J. Bishop, M. Bey, W. Anderst, and S. Tashman. A study of the response of the human cadaver head to impact. Stapp Car Crash Journal, 51:17-80, 2007. 
[258] M-C. Lee and R. C. Haut. Insensitivity of tensile failure properties of human bridging veins to strain rate: Implications in biomechanics of subdural hematoma. Journal of Biomechanics, 22(6):537 - 542, 1989.

[259] P. Parizel, S. Makkat, E. Van Miert, J. Van Goethem, L. van den Hauwe, and A. M. De Schepper. Intracranial hemorrhage: Principles of ct and mri interpretation. European radiology, 11:1770-83, 022001.

[260] C. Rambaud. Bridging veins and autopsy findings in abusive head trauma, 2015.

[261] B. M. Knowles, S. R. MacGillivray, J. A. Newman, and C. R. Dennison. Influence of rapidly successive head impacts on brain strain in the vicinity of bridging veins. Journal of Biomechanics, 59:59-70, jul 2017.

[262] R. H. Pudenz and C. H. Shelden. The Lucite calvariumâa Method for direct observation of the brain. Journal of Neurosurgery, 3(6):487-505, 1946.

[263] K. L. Monson, M. I. Converse, and G. T. Manley. Cerebral blood vessel damage in traumatic brain injury. Clinical Biomechanics, 64:98-113, 2019.

[264] C. Brockmann, S. Kunze, and J. Scharf. Computed tomographic angiography of the superior sagittal sinus and bridging veins. Surgical and Radiologic Anatomy, 33(2):129-134, 2011.

[265] G. F. J. Migueis, F. A. O. Fernandes, M. Ptak, M. Ratajczak, and R. J. Alves de Sousa. Detection of bridging veins rupture and subdural haematoma onset using a finite element head model. Clinical Biomechanics, 63:104-111, 2019.

[266] G. U. Musigazi and B. Depreitere. Study of bridging vein anatomy based on ct angiogram data for optimization of finite element models simulating acute subdural hematoma, 2015.

[267] M. Kapeliotis, G. U. Musigazi, N. Famaey, B. Depreitere, S. Kleiven, and J. Vander Sloten. The sensitivity to inter-subject variability of the bridging vein entry angles for prediction of acute subdural hematoma. Journal of Biomechanics, In press, may 2019. 
[268] P. Wahl, N. Schreyer, and B. Yersin. Injury pattern of the Flash-Ball ${ }^{\circledR}$, a less-lethal weapon used for law enforcement: Report of two cases and review of the literature. Journal of Emergency Medicine, 2006.

[269] A. Oukara, N. Nsiampa, C. Robbe, and A. Papy. Assessment of NonLethal Projectile Head Impacts. Human Factors and Mechanical Engineering for Defense and Safety, 2017.

[270] R. Millar, W. H. Rutherford, S. Johnston, and V. J. Malhotra. Injuries caused by rubber bullets: A report on 90 patients. British Journal of Surgery, 1975.

[271] J. Hiss, F. N. Hellman, and T. Kahana. Rubber and Plastic Ammunition Lethal Injuries: The Israeli experience. Medicine, Science and the Law, 1997.

[272] A. Mahajna, N. Aboud, I. Harbaji, A. Agbaria, Z. Lankovsky, M. Michaelson, D. Fisher, and M. M. Krausz. Blunt and penetrating injuries caused by rubber bullets during the Israeli-Arab conflict in October, 2000: A retrospective study. Lancet, 2002.

[273] J. Hiquet, E. Christin, L. Benali, and S. Gromb-Monnoyeur. La dangerosité du Flash-Ball ${ }^{\circledR}$ : Cas rapporté d'un traumatisme crânioencéphalique et revue de la littérature. Revue de Medecine Legale, 2016.

[274] V. Pinaud, P. Leconte, F. Berthier, G. Potel, and B. Dupas. Orbital and ocular trauma caused by the Flash-Ball ${ }^{\circledR}$ : A case report. Injury Extra, 2009.

[275] P. M. Brun, J. Bessereau, H. Chenaitia, C. Barberis, and M. Peyrol. Commotio cordis as a result of neutralization shot with the Flash Ball ${ }^{\circledR}$ less-lethal weapon, 2012.

[276] C. Robbe, N. Nsiampa, A. Papy, and A. Oukara. An hybrid experimental/numerical method to assess the lethality of a kinetic energy non-lethal weapon system. 27th International Symposium on Ballistics, 2013.

[277] C. Bir and D. C. Viano. Design and injury assessment criteria for blunt ballistic impacts. Journal of Trauma - Injury, Infection and Critical Care, 2004. 
[278] C. Deck and R. Willinger. Head injury prediction tool for protective systems optimisation. In 7th European LS-DYNA Conference, 2009.

[279] J. Hallquist. LS-DYNA ${ }^{\circledR}$ theory manual. 2006.

[280] L. E. Schwer, S. W. Key, T. A. Pučik, and L. P. Bindeman. An assessment of the LS-DYNA hourglass formulations via the 3D Patch Test. 5th European LSDYNA users conference, 2005.

[281] Livermore Software Technology Corporation. LS-DYNA Keyword User's Manual Volume II Material Models. 2016. 